\title{
The Contribution of Cognitive Impulsivity to Criminal Risk
}

\author{
By \\ Rebecca K. Bell
}

\begin{abstract}
A thesis submitted to Victoria University of Wellington in fulfillment of the requirements for the degree of Doctor of Philosophy in Psychology
\end{abstract}

Victoria University of Wellington

2013 


\section{Acknowledgments}

Wow, what a journey; the most challenging project of my life so far, and one I could not have conquered without the support of many special people. To my primary supervisor, Devon Polaschek, I will forever be indebted to you for the experiences and opportunities you have given me over the years. If it weren't for your faith in me, I would never have taken the leap to complete a PhD in the first place! You have been incredibly patient and supportive. What an enormous privilege to have spent time in your lab and to move forward from here taking the benefits of your input and feedback — both personally and professionally—with me.

To my secondary supervisor, Gina Grimshaw, I can't describe how valuable your help with all things "cognitive" has been! You have sparked my interest further in an area that I never thought I would enjoy so much. To Marc Wilson, your help with statistics allowed me to uncover the most interesting story my data had to tell. Thank you so much for taking the time to help me in amongst all of your other jobs (and for soothing my moments of stats panic!).

To the therapy and custodial staff of the STURPs across New Zealand: thank you for your willingness to assist the research and for making a stray student from Wellington feel so welcome in your units. To the participants in this thesis: without you, I could not have completed this piece of work. You will probably never know how much I enjoyed our research sessions; every visit to the prison was a reminder of why I love my job. I consider it a huge privilege that you were willing to let me share time with you. 
To the "Labsters": what a superb bunch of people. Your smarts (and superb fashion sense) never cease to amaze me. I will never forget the group effort for my "coffee date" or watching your rendition of "Gangnam Styles" in our conference hotel room! You are all rock stars; always have faith in the top-notch skills and the special souls that you have.

To my Mum and Dad: saying thank you just doesn't cut it. You taught me to have the discipline and drive to succeed that meant I could (finally) get through this $\mathrm{PhD}$ in one piece. To Mum: if someone could bottle the infinite love and support you give me, the world would be a better place. To Dad: your pride in me kept me going. I'm sure we all never thought I would still be at "school" for this long, but let's hope that for every time you bailed me out when money was tight, or consoled me when I felt stressed, that I can give you a special return on your investment by continuing to make you proud.

To my very special fiancé, Grant: what would I do without you? You embrace the things that I am passionate about like they are your own dreams. You recite the title of my thesis like you are just as proud as I am to be doing this work. Every wall I felt I hit, you helped me to knock it down and plough through by believing in me. Nothing is ever too hard when you are beside me. 


\begin{abstract}
Impulsivity increases risk for general, violent and sexual offending. Accordingly, helping offenders to become better regulators of their impulses is one goal of offender rehabilitation. In a correctional setting, the assessment of impulsivity focuses on personality and behaviour, but not cognition; cognitive impulse control impairments are inferred from personality styles and behavioural patterns suggestive of acting before thinking. However, empirical findings challenge the validity of inferring cognition from personality and behavioural measures. Additionally, without assessing cognition, practitioners are limited in their ability to isolate which cognitive processes are most impaired and therefore worthy of intervention for individual offenders.

To establish the contribution of cognitive impulse control to criminal risk, a theoretically derived, empirically supported neurocognitive assessment framework was adopted. The framework is based on the notion that impulsive behaviour arises from three, potentially dissociable skill domains that support impulse control: decisionmaking, perceptual and motor impulse control. A cohort of 77 men attending intensive cognitive-behavioural rehabilitation was recruited from four of New Zealand's prisonbased Special Treatment Unit Rehabilitation Programmes (STURPs). A neurocognitive battery of five tasks collectively representing each cognitive impulse control domain was administered before and after the 8-month treatment programme.

Study One explored pre-treatment clinically impaired performance within and across each cognitive impulse control domain. Compared to normative data, performance was typically in the average to below average range, but it was not clinically impaired
\end{abstract}


overall. When performance was clinically impaired, it was most pronounced on tasks requiring cognitive flexibility.

Study Two explored treatment change in cognitive impulse control. The study also compared pre-treatment cognitive impulse control between offenders who went on to complete the treatment programme and those who were prematurely removed for responsivity or conduct-related issues. No pre-treatment cognitive impulse control differences were found between treatment completers and non-completers. Treatment completers displayed small pre-post treatment improvements in some areas of cognitive impulse control, but not others.

Study Three explored cross-sectional and predictive relationships between cognitive impulse control, dynamic criminal risk, trait anger and anger control. Although there was little association between these variables before treatment, some cognitive impulse control outcomes predicted post-treatment dynamic criminal risk, trait anger and anger control. Thus, the evidence suggested that certain aspects of cognitive impulse control might function as facilitators of treatment change.

Together, the findings highlighted the importance of evaluating cognitive impulse control as part of the risk assessment, and clinical formulation process. The findings also suggested that interventions designed to develop cognitive impulse control abilities either before, or as a complement to traditional cognitive-behavioural interventions, have the potential to maximise treatment response. 


\section{List of common abbreviations used throughout this thesis}

$\begin{array}{ll}\text { STURPs } & \text { Special Treatment Unit Rehabilitation Programmes } \\ \text { IGT } & \text { Iowa Gambling Task } \\ \text { D-KEFS } & \text { Delis Kaplan Executive Function System } \\ \text { CWIT } & \text { Colour-word Interference Test } \\ \text { CTMT } & \text { Comprehensive Trail-making Test } \\ \text { CPT } & \text { Continuous Performance Test } \\ \text { VRS } & \text { Violence Risk Scale } \\ \text { VRS-SO } & \text { Violence Risk Scale - Sex Offender Version } \\ \text { STAXI-II } & \text { State-Trait Anger Expression Inventory } \\ \text { RT } & \text { Response/reaction Time } \\ \text { SSRT } & \text { Stop Signal Reaction Time } \\ \text { ms } & \text { Milliseconds }\end{array}$




\section{Table of Contents}

Acknowledgements 2

$\begin{array}{ll}\text { Abstract } & 4\end{array}$

List of common abbreviations $\quad 6$

Table of Contents

List of Tables $\quad 14$

List of Figures $\quad 16$

Chapter 1: What is impulsivity and why does it matter?

$\begin{array}{ll}1.1 \text { Introduction } & 17\end{array}$

1.2 Different approaches to defining and assessing impulsivity 17

1.3 Behavioural, personality and cognitive approaches to defining and 19

assessing impulsivity

1.3.1 The behavioural approach 19

1.3.2 The personality approach 23

1.3.3 The cognitive approach 27

1.3.3.1 Decision-making definitions of impulsivity

1.3.3.1.1 Reward sensitivity 28

1.3.3.1.2 Response perseveration 29

1.3.3.2 Perceptual impulse control definitions of impulsivity 32

1.3.3.3 Motor impulse control definitions of impulsivity 33

1.3.4 Defining and assessing impulsivity: summary 36

Chapter 2: Impulsivity and antisocial behaviour across the lifespan

2.1 Background 37

2.2 The developmental relationship between impulsivity and antisocial 37

risk

2.2.1 Moffitt's developmental taxonomy 38

2.2.1.1 Summary of Moffitt's developmental taxonomy 40

2.2.1.2 Empirical support for the LCP theory during childhood and 41

adolescence 
2.2.1.2.1 Summary of child and adolescent studies

2.2.1.3 Empirical support for the LCP theory during adulthood 45

2.2.1.4 Cognitive impulse control in adult offenders 47

2.2.1.4.1 Summary of cognitive impulse control in adult offenders 50

2.3 Advantages of the cognitive approach 51

2.4 The importance of assessing cognitive impulse control in adult 55

offender samples

2.5 Where to from here?

Chapter 3: Bechara's framework: A dual systems perspective

3.1 Impulsive and reflective systems 61

3.2 The impulsive system $\quad 61$

3.3 The reflective system $\quad 63$

3.3.1 Mechanisms of weakness in the reflective system: Decision-making, $\quad 66$ perceptual and motor impulse control

3.4 Summary and research objectives $\quad 67$

$\begin{array}{ll}3.5 \text { Thesis structure } & 68\end{array}$

3.6 Study outlines and specific research question 69

Chapter 4: General Method

4.1 Setting

4.2 Participants

4.2.1 Offender sample $\quad 74$

4.2.2 University student sample $\quad 74$

$\begin{array}{ll}4.3 \text { Materials } & 75\end{array}$

4.3.1.1 Decision-making domain: Iowa Gambling Task (IGT) 76

4.3.1.2 IGT outcome scores

$\begin{array}{ll}\text { 4.3.1.2.1 Total net scores } & 79\end{array}$

4.3.1.2.2 Block net scores $\quad 80$

4.3.1.2.3 Card selections from each deck 81 
$\begin{array}{ll}\text { 4.3.4.2 CPT reliability } & 88\end{array}$

$\begin{array}{ll}\text { 4.3.5 Stop Signal Task } & 88\end{array}$

4.3.5.1 Stop signal outcome scores $\quad 90$

4.3.5.2 Stop signal reliability 91

4.3.6 The Wechsler Abbreviated Scale of Intelligence 91

4.3.7 Violence Risk Scale 92

$\begin{array}{ll}4.4 \text { Procedure } & 94\end{array}$

Chapter 5: Is cognitive impulse control intact or impaired in a sample of high-risk offenders?

5.1 Introduction 96

5.2 Study objectives and hypotheses 98

$\begin{array}{ll}5.3 \text { Results and discussion } & 100\end{array}$

5.3.1 Rationale for selection of outcome scores from each task 101

5.3.2 Principal components analysis (PCA) results 103

5.3.2.1 PCA summary 106

5.3.3 Exploring clinically impaired performance 106 
5.3.3.1 Decision-making performance

5.3.3.2 Perceptual impulse control performance: CWIT

5.3.3.3 Perceptual impulse control performance: CTMT

5.3.3.4 Motor impulse control performance: $C P T$

5.3.3.4.1 General performance indicators

5.3.3.4.2 Impulsive performance indicators

5.3.3.5 Motor impulse control performance: Stop Signal Task

5.3.3.6 Proportions of impairment across domains

5.4 General discussion

5.4.1 Empirical support for Bechara's framework

5.4.2 Evidence against clinically impaired cognitive impulse control overall

5.4.3 Specificity of impairment: exploring the unique complexities of each task

5.5 Summary

Chapter 6: Changes in cognitive impulse control following treatment

6.1 Introduction 135

6.2 The treatment context 136

6.3 Plasticity of the adult brain: training-related changes to neural 137 structures and cognitive functioning

6.4 The treatment context: more opportunity to practice impulse control, or more opportunity to fail?

6.5 Study objectives and hypotheses 142

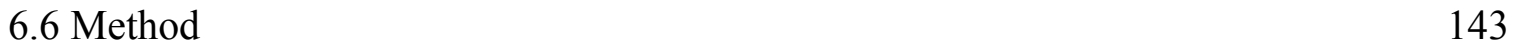

6.6.1 Statistical analyses 143 
6.6.3 Procedure 144

$\begin{array}{ll}6.7 \text { Results } & 144\end{array}$

6.7.1 Decision-making performance: Iowa Gambling Task (IGT) 145

6.7.1.1 Deck $A \quad 147$

$\begin{array}{ll}\text { 6.7.1.2 Deck } B & 148\end{array}$

6.7.1.3 Deck C 148

6.7.1.4 Deck D 149

6.7.1.5 IGT decision-making performance summary 149

6.7.2 Perceptual Impulse Control: Colour Word Interference Test (CWIT) 150

6.7.2.1 CWIT raw completion time and error summary 151

6.7.3 Perceptual Impulse Control: Trail Making Test (CTMT) 152

6.7.4 Motor Impulse Control: Continuous Performance Test (CPT) 154

6.7.5 Motor Impulse Control: Stop Signal 155

6.7.6 General summary: patterns of change 156

6.7.7 Comparison of pre-treatment cognitive impulse control between 157

treatment completers and non-completers

6.8 General discussion 159

6.8.1 Small, selective cognitive impulse control changes 159

6.8.2 Explanations for small post-treatment changes 160

6.8.3 Theoretical and clinical implications of changes (or lack of) 162

6.8.4 No differentiation between treatment completers and non- 164

completers

6.8.5 Cognitive impulse control as a responsivity factor?

Chapter 7: Cognitive impulse control, criminal risk, and anger

$\begin{array}{ll}7.1 \text { Introduction } & 166\end{array}$

7.2 How might cognitive impulse control be related to criminal risk, trait 167 anger and anger control?

7.3 Study objectives and hypotheses 
7.4 Method

$\begin{array}{ll}\text { 7.4.1 Participants } & 172\end{array}$

7.4.2 Materials 172

7.4.2.1 State-Trait Anger Expression Inventory 173

$\begin{array}{ll}\text { 7.4.3 Procedure } & 174\end{array}$

$\begin{array}{ll}\text { 7.4.4 Data analysis } & 175\end{array}$

7.5 Results and discussion 176

7.5.1 Pre and post-treatment change in VRS static, dynamic and total risk 177

7.5.2 Pre and post-treatment change in STAXI trait anger and anger 177

control

$\begin{array}{ll}\text { 7.5.3 Pre-treatment correlations } & 178\end{array}$

7.5.4 Cross-lag correlations $\quad 179$

7.5.4.1 Cognitive impulse control and VRS dynamic risk 180

7.5.4.1.1 Summary of cognitive impulse control and VRS dynamic risk 182

7.5.4.2 Cognitive impulse control and STAXI trait anger 182

7.5.4.2.1 Summary of cognitive impulse control and STAXI trait anger 183

7.5.4.3 Cognitive impulse control and STAXI anger control 184

7.5.4.3.1 Summary of cognitive impulse control and STAXI anger control 185

$\begin{array}{ll}7.6 \text { General discussion } & 186\end{array}$

7.6.1 Decision-making, inhibition and dynamic violence risk 187

7.6.2 Decision-making, inhibition, set shifting, trait anger and anger 189 control

7.7 Summary

\section{Chapter 8: General Discussion}

8.1 Revisiting thesis aims and chapter overview 193

8.2 Theoretical implications 195

8.2.1 Chronically weak reflective systems: Lack of empirical support 195

8.2.2 Different explanations for dysfunctional impulsive behaviour: 198

cognitive versus personality impulsivity 
8.2.2.1 Two non-impulsive types to consider 203

8.2.3 Developmental trajectories of cognitive and personality impulsivity 204

8.2.4 The interplay between cognitive impulse control, criminal risk and 209 emotion regulation

8.2.4.1 Cognitive impulse control and dynamic criminal risk 209

8.2.4.2 Cognitive impulse control, anger and anger control 211

8.3 Clinical implications 213

8.3.1 Risk assessment and clinical formulation 213

8.3.2 Specifying mechanisms of change for cognitive interventions 214

8.3.3 Empirical support for cognitive interventions 216

8.3.4 Summary of clinical implications 218

8.4 Limitations and key areas for future investigation 219

8.4.1 Inclusion of personality impulsivity and other measures 219

8.4.2 Larger sample sizes for increasing statistical power in existing 221

analyses and recidivism analyses

8.4.3 Manipulation of assessment context and experimental stimuli 221

8.4.4 Inclusion of non-treated, matched control group 225

8.4.5 Comparisons of cognitive impulse control between offender sub- 226

groups

$\begin{array}{ll}\text { 8.5 Summary } & 228\end{array}$

$\begin{array}{ll}\text { References } & 229\end{array}$

$\begin{array}{ll}\text { Appendices } & 282\end{array}$ 


\section{List of Tables}

\section{Table 1}

Pay-off scheme for each IGT card deck

Table 2

Outcome scores from each task within Bechara's three domains of

cognitive impulse control

\section{Table 3}

Rotated factor loadings for 9 outcome scores selected for each cognitive impulse control domain

\section{Table 4}

IGT raw total and block net scores with normative comparisons

\section{Table 5}

Proportion of participants with net scores in the clinically impaired

range

\section{Table 6}

Mean number of card selections from each deck per block of 20 cards

\section{Table 7}

CWIT mean raw completion time, scaled and contrast scores

Table 8

CTMT mean raw completion time and normative comparisons

Table 9

CPT mean raw scores and normative comparisons

\section{Table 10}

Offender and student raw score comparisons on the Stop Signal task

\section{Table 11}

Pre-to post-treatment comparison of raw completion time and errors on the CWIT

\section{Table 12}

Pre- to post-treatment comparison of raw completion times on the CTMT 


\section{Table 13}

Comparison of pre-treatment cognitive impulse control between

treatment completers and non-completers

\section{Table 14}

Pre and post-treatment VRS risk (dynamic, static, total), STAXI trait

anger, and STAXI anger control

\section{Table 15}

Interpretive guide for direction of cognitive impulse control, dynamic

criminal risk and anger outcomes

\section{Table 16}

Pre-treatment correlations between cognitive impulse control, VRS

dynamic criminal risk, STAXI trait anger and STAXI anger control 


\section{List of Figures}

\section{Figure 1}

Pre-treatment mean number of cards selected from each IGT deck

\section{Figure 2}

Post-treatment mean number of cards selected from each IGT deck

\section{Figures 3-10}

Cross-lag correlations: Cognitive impulse control and VRS dynamic risk

$180-181$

\section{Figure 11-18}

Cross-lag correlations: Cognitive impulse control and STAXI trait anger

$182-183$

\section{Figures 19-26}

Cross-lag correlations: Cognitive impulse control and STAXI anger

$184-185$

control 


\section{Chapter 1}

\section{What is impulsivity and why does it matter?}

\subsection{Introduction}

To live adaptively, often we must control urges to act on immediate impulses if those impulses are inconsistent with longer-term desires. For example, inhibiting the impulse to reach for a piece of chocolate cake is important when pursuing a weight loss goal; and inhibiting frustration at others can be important for maintaining healthy relationships. Sometimes acting on impulse is positive: it can be invigorating to live for the moment without concern for tomorrow. But sometimes, impulsive behaviour may not be the same behaviour we would choose had we spent longer considering response options. For some people, chronic failure to inhibit impulsive behaviour substantially interferes with their ability to live adaptively. Specifically, this thesis explores how acting on impulse contributes to risk of criminal behaviour. To begin, this chapter outlines the common definitions and assessment approaches of impulsivity adopted in the clinical and forensic literatures. The complexity of the impulsivity construct denotes that definitions and corresponding assessment methods can capture behaviour, personality or cognition: definitions and assessment methods derived from each type of approach are outlined below.

\subsection{Different approaches to defining and assessing impulsivity}

Defining impulsivity is a complex task. Put simply, impulsivity is the characteristic we infer to be the cause of behaviour that - to the outside observer-looks 
poorly planned and rapidly executed (Evenden, 1999). Poorly planned, rapidly executed behaviour is sometimes adaptive, with more favourable outcomes than for careful deliberation (e.g., braking quickly when the car in front of yours stops suddenly). But acting without thinking can also be maladaptive. Dickman (1990) differentiated between functional and dysfunctional types of impulsivity. Functional impulsivity occurs when the consequences of action without forethought are adaptive (e.g., braking quickly). In contrast, dysfunctional impulsivity occurs when the consequences of action without forethought are maladaptive (e.g., spending money on a spontaneous shopping spree rather than paying overdue bills). Although functional impulsivity is worthy of investigation to further our understanding of the many dimensions and manifestations of impulsivity, dysfunctional impulsivity is the central focus of this thesis because it is an important risk factor for criminal behaviour (Wong \& Gordon, 2000).

Clinically, dysfunctional impulsivity is elevated in populations with Attention Deficit/Hyperactivity Disorder (ADHD; Overtoom, Kenemans, Verbaten, Kemmer, van der Molen \& van Engeland, 2002; Winstanley, Eagle \& Robbins, 2006), substance use disorders (Bornovalova, Daughters, Hernandez, Richards \& Lejuez, 2005; Finn, Mazas, Justus \& Steinmetz, 2002), a history of suicide attempts and self-mutilation (Winchel \& Stanley, 1991; Wyder \& De Leo, 2007; Zouk, Tousignant, Seguin, Lesage \& Turecki, 2006), psychopathy (Patrick, Fowles \& Krueger, 2009), and it is a symptom of Obsessive-Compulsive Disorder, Borderline Personality Disorder and Antisocial Personality Disorder (American Psychiatric Association [DSM-IV-TR], 2000). Perhaps the most costly form of dysfunctional impulsivity from a legal, financial and social perspective is that which contributes to the development and maintenance of antisocial 
behaviour (Andrews \& Bonta, 2006). Specifically, impulsivity is a dynamic risk factor that increases risk for general, violent and sexual offending (Andrews, Bonta \& Wormith; 2006; Wong \& Gordon, 2000). The dynamic nature of impulsivity means that it is amenable to change. Accordingly, assisting offenders to become better regulators of their impulses is a common treatment target for offender rehabilitation (Andrews \& Bonta, 2006).

\subsection{Behavioural, personality and cognitive approaches to defining and assessing impulsivity}

\subsubsection{The behavioural approach}

Evenden (1999) aptly captures the behavioural approach by defining impulsivity as "a range of actions which are poorly conceived, prematurely expressed, unduly risky or inappropriate to the situation that often results in undesirable consequences" (p.348). Thus, behavioural definitions of impulsivity are non-specific because they refer to any act that looks like it occurred automatically, without forethought and ended badly. It is important to note that although isolated behaviours can be impulsive, dysfunctional conceptualisations of impulsivity presume that the individual has a predisposition toward frequent displays of impulsive behaviour, rather than single acts of impulsivity (Moeller et al., 2001). Dysfunctional impulsive behaviours are typically considered destructive, and include but are not limited to, excessive substance use, driving recklessly and unplanned sexual relationships (Eaves, Tien \& Wilson, 1997).

Behaviourally based assessment methods involve analysing general trends in behaviour across time and context. Information from structured or semi-structured interviews with an individual and information from collateral sources is evaluated to 
judge whether impulsivity represents a clinically significant problem. In the published clinical literature, the Lifetime History of Impulsive Behaviours Interview (LHIB; Schmidt, Fallon, \& Coccaro, 2004) and Lecrubier's Impulsivity Rating Scale (IRS; Lecrubier, Braconnier, Said \& Payan, 1995) appear to be the only clinician-rated, adult, behaviourally based assessment measures exclusively dedicated to impulsivity.

The LHIB assesses the functional or dysfunctional nature of impulsivity, the preplanned or impulsive nature of behaviour, clinically relevant impulsive behaviour, and actual behaviours (including motivations and circumstances), which have occurred in real-life settings. The IRS captures multiple components of impulsivity including: irritability (e.g., losing temper), patience-impatience (e.g., capacity to wait), time to make decisions (e.g., buying habits in department stores), capacity to pursue an activity (e.g., ability to pursue or tendency to be distracted), aggressiveness (e.g., aggressive response to unpleasant situations), ability to control responses, and capacity for delay. On the IRS, impulsivity is rated according to the day-to-day behaviour of an individual during the week preceding assessment. Studies comparing clinical and non-clinical subjects have illustrated that both the LHIB and the IRS demonstrate good reliability and validity; but studies using these measures with offenders are limited (Lecrubier, Braconnier, Said \& Payan, 1995; Schmidt, Fallon, \& Coccaro, 2004).

Examples of impulsivity definitions specific to a correctional setting come from criminal risk instruments. Criminal risk instruments utilise the behavioural approach by considering recent and historical behaviour — criminal or otherwise — to make a judgement about the contribution of impulsivity to an offender's criminal risk. For example, the Levels of Service-Case Management Inventory defines impulsivity as a 
specific attribute and personal problem with criminogenic potential characterised by a pattern of non-reflective, impulsive action (LS-CMI; Andrews, Bonta \& Wormith, 2004). The Violence Risk Scale (VRS; Wong \& Gordon, 2000) operationalises impulsivity as a typical style of reacting to situations or provocations; a style that relates to an offender's general behaviour, not just behaviour associated with criminal acts or violence.

According to the VRS, the impulsive individual is likely to "do or say things on the spur of the moment, or to act now and think later" (VRS manual; Wong \& Gordon, 2000, p.64). The Massachusetts Treatment Centre Classification System assesses lifestyle impulsivity on the basis of general functioning including: employment history, financial irresponsibility, aimlessness, reckless behaviour, inability to maintain enduring attachments, repeated instances of aggressive or destructive behaviour in response to frustration, and subjective experiences of acting on irresistible impulses, whims or urges (Prentky \& Knight, 1986). Hare's Psychopathy Checklist-Revised (Hare 1991, 2003), views impulsivity as contributing to a socially deviant lifestyle (Factor 2). Specifically, an impulsive individual is "unlikely to spend much time weighing the pros and cons of a course of action, or in considering the possible consequences of his actions to oneself or others" (PCL-R manual; Hare, 1991, 2003, p.43). According to the PCL-R, such spur of the moment action is expressed behaviourally as suddenly breaking off relationships, quitting a job without another job to go to, and moving from place to place on little more than a whim and without bothering to inform others (Hare, 1991, 2003).

The Sex Offender Need Assessment Rating (SONAR; Hanson \& Harris, 2001) assesses the extent to which an offender is easily bored, seeks thrills and has little regard for personal safety or the safety of others. The STABLE-2000 and STABLE-2007 
(Hanson, Harris, Scott \& Helmus, 2007) conceptualise impulsivity as one aspect of general self-regulation: an “offender's ability to self-monitor and inhibit antisocial thoughts and behaviours" (STABLE scoring guide, p.41). Similar to the PCL-R, the STABLE conceptualises impulsive acts as behaviours that have a high likelihood of negative consequences: reckless driving, substance abuse, partying, accepting bets and dares, quitting jobs with no others in sight, changing residences, unsafe work practices and starting fights. In addition, across multiple contexts, the impulsive individual is easily bored, seeks thrills and has little regard for personal and others' safety (STABLE scoring guide, p. 42). According to the STABLE, poor cognitive problem solving and negative emotionality or hostility underpins destructive impulsive behaviour.

The Historical, Clinical and Risk Management Violence Risk Assessment Scheme (HCR-20; Webster, Douglas, Eaves \& Hart, 1997b) also captures the role of negative emotionality in its description of impulsivity as marked by behavioural and affective instability. Indicators of impulsivity in the HCR-20 include: dramatic hour-to-hour, dayto-day fluctuations in mood and general demeanour, an inability to remain composed and directed even when under pressure to act, and "hair trigger" reactions particularly to real or imagined insults and disappointments (HCR-20 manual, p.75). Both the STABLE and HCR-20 highlight the interplay between cognitive and emotional processes, suggesting that impulse control is related to emotion regulation specifically, and to more general behavioural control.

In summary, clinical and forensic behavioural assessments of impulsivity are based on clinical judgement of a person's typical interactional style with other people and the world. A typical interactional style indicative of action without forethought- 
particularly under negative emotional load — culminating in maladaptive outcomes, is considered evidence for dysfunctional impulsivity.

\subsubsection{The personality approach}

From the personality approach, impulsivity is operationalised as an enduring

personality trait (Lane \& Cherek, 2000). The location of impulsivity within a general model of personality is contested. For example, whilst Barratt (1993) considered impulsivity orthogonal to neuroticism, Costa and McCrae (1992) placed their impulsiveness facet within their neuroticism domain (Whiteside \& Lynam, 2001). Although it is largely agreed that the personality construct of impulsivity is multifactorial, the number of factors varies. Eysenck and Eysenck (1977) found that impulsivity could be subdivided into four factors - narrow impulsiveness, risk-taking, non-planning and liveliness - each of which showed distinct associations with neuroticism and psychoticism. Barratt (1985) found three: cognitive (making quick decisions), motor (acting without thinking), and non-planning (lack of forethought) impulsivity. Buss and Plomin (1975) suggested that inhibitory control is central to impulsivity but that decision time, persistence, boredom and sensation seeking are also important dimensions. In a psychobiological model, Cloninger, Svrakic and Przybeck (1993) proposed that impulsivity is one aspect of novelty seeking, which also includes initiating approach behaviour in response to novelty, extravagance in approach to reward cues and the tendency to quickly lose one's temper.

Impulsive personality is typically assessed using self-report questionnaires with multiple subscales to capture the different dimensions or factors of impulsive personality. Self-report assessments are based on the premise that knowledge about our own 
impulsiveness is consciously accessible (Enticott \& Ogloff, 2006). Therefore, the reliability of self-report methodologies partly depends on the accuracy of an individual's insight into how, and how often they are impulsive. In a correctional setting, self-report questionnaires are either solely dedicated to impulsivity (e.g., Barratt Impulsiveness Scale; BIS-11, Patton, Stanford \& Barratt, 1995), or contain subscales that assess impulsivity-related traits and tendencies (e.g., Self-control subscale: Antisocial Personality Questionnaire, Blackburn \& Fawcett, 1999; Control/Impulsiveness subscale: Multidimensional Personality Questionnaire, Tellegan, 1982; Self-control Subscale: California Psychological Inventory, Gough, 1975; Restraint Subscale: GuilfordZimmerman Temperament Survey, Guilford, Zimmerman, \& Guilford, 1976; Impulse Expression subscale: Basic Personality Inventory, Jackson, 1989; Impulse control subscale: Big Five Questionnaire, Caprara, Barbaranelli, Borgogni \& Perugini, 1993). In their most recent forms, the Barratt Impulsiveness Scale, Version 11 (BIS-11; Patton, Stanford \& Barratt, 1995) and the Eysenck Impulsiveness, Venturesomeness, and Empathy Questionnaire ( $\mathrm{I}_{7}$; Eysenck, Pearson, Easting \& Allsopp, 1985) are two selfreport measures commonly used in offender populations, although they were not exclusively designed for this purpose. The BIS-11 has 30 items based on a 3 -factor model: 1) Attentional impulsiveness ( 8 items; e.g., "I am restless at the theatre or lectures"), 2) Motor impulsiveness (11 items; e.g., "I act on the spur of the moment"), and 3) Non-planning impulsiveness (11 items; e.g., "I am (not) a careful thinker"). The BIS-11 has been validated in healthy, psychiatric and offender populations (e.g., Echeburua \& Fernandez- Montalvo, 2007; Luengo, Carrillo de-la-Pena, Otero, \& Romero, 1994; Mobini, Grant, Kass, \& Yeomans, 2007; Parry \& Lindsay, 2003; Smith \& 
Waterman, 2004), although some authors question the factor structure of the instrument when used with offenders.

For example, Haden and Shiva $(2008,2009)$ conducted two studies in large samples of male forensic inpatients. They failed to replicate the original 3-factor structure; instead finding a better fit with a 2-factor model, consisting of non-planning and physical restlessness/impulsive action factors. Ireland and Archer (2008) found a 3factor model similar to Patton and colleagues' original model but the nature of each factor was slightly different, consisting of non-planning, behavioural and concentration/distractibility. Using exploratory factor analysis, Ruiz, Skeem, Poythress, Douglas and Lilienfeld (2010) found a 3-factor model that looked similar to Patton and colleagues' structure, but confirmatory factor analysis failed to replicate the model. Ruiz and colleagues were also unable to replicate Haden and Shiva's 2-factor model. Overall, Ruiz and colleagues concluded that the factor structure of the BIS-11 is inconclusive when administered using a sample of North American, incarcerated offenders.

The Eysenck Impulsiveness, Venturesomeness, and Empathy Questionnaire assesses two broad impulsivity dimensions - impulsiveness and venturesomeness - using 54 items. The impulsiveness subscale (19 items; e.g., "I often buy things on impulse") operationalises impulsivity as behaving without thinking and without realising the risks involved. The venturesomeness subscale (16 items; e.g., "do you sometimes like doing things that are a bit frightening?") operationalises venturesomeness as acting despite awareness of the risks involved. Impulsiveness subscale scores have been shown to correlate with measures of violent behaviour $(r=.54)$, PCL-R psychopathy $(r=.52)$, 
assault convictions $(r=.37)$ and self-reported fights $(r=.44$; Seager, 2005; also see Retz, Retz-Junginger, Supprian, Thome \& Rosler, 2004).

Factor analysis of scores from other questionnaires capturing impulsiveness and venturesomeness — sometimes labelled sensation seeking — typically support a two, rather than one-factor solution (e.g., Cyders \& Coskunpinar, 2012; Magid, MacClean \& Colder, 2007). The better fit for a two-factor solution illustrates an important difference that is sometimes blurred in the literature: between behaviour that is caused by action without forethought versus purposeful behaviour that occurs despite the risks involved (Dolan \& Fullam, 2004; Magid, Maclean and Colder, 2007).

The Grasmick Self-control Scale has also been used with offender populations, though not as frequently as the BIS-11 and $\mathrm{I}_{7}$ (Grasmick, Tittle, Bursick \& Arneklev, 1993). The Self-control Scale is the based on Gottfredson and Hirschi's (1990) assertion that deficient self-control and opportunity motivate deviancy. The scale has a total of 24 items derived from a 6-factor conceptualisation of self-control. The 6 factors of selfcontrol include: impulsivity (e.g., "I often act on the spur of the moment"), simple tasks (e.g., "I frequently try to avoid projects that I know will be difficult"), risk seeking (e.g., "sometimes I will take a risk just for the fun of it"), physical activities (e.g., "I almost always feel better when I am on the move than when I am sitting and thinking"), selfcenteredness (e.g., "if things I do upset people, it's their problem not mine"), and temper (e.g., often when I'm angry at people, I feel more like hurting them than talking to them about why I am angry"; Grasmick et al., 1993). Longshore, Turner and Stein (1996) administered the Self-control Scale to a heterogeneous offender sample. Using factor analysis, they identified five subscales that were relatively consistent with Gottfredson 
and Hirschi's conceptualisation of self-control. Total scale scores and some subscales scores were moderately associated with self-reported fraud and force offences (Longshore et al., 1996).

To summarise the personality approach, impulsivity as a personality construct is multidimensional with between two and four overarching factors that broadly capture traits indicative of acting before thinking and preference for risky behaviour. Self-report questionnaires differ in the number of factors they include within those two broad dimensions, as well as the labels they adopt for each factor.

\subsubsection{The cognitive approach}

The cognitive approach defines impulsivity according to the cognitive processes that facilitate the expression of impulsive behaviour. Accordingly, confusion often arises in the psychological literature because so many cognitive causes are nominated; and they are not necessarily mutually exclusive. Moreover, different causes can represent redescriptions of the same underlying process, or describe the by-product of a single process (Enticott \& Ogloff, 2006). For example, emphasis on the present will inevitably lead to failure to consider longer-term consequences of behaviour (Enticott \& Ogloff, 2006). Nevertheless, the advantage of assessing impulsivity from the cognitive approach is that behavioural responses during cognitive tasks are made under experimentally controlled conditions. Experimental control permits more reliable isolation of cognitive causes for behaviour than is possible from evaluating naturalistic behaviours that are not experimentally controlled.

One useful way to organise the many cognitive definitions and corresponding assessment methods is via Bechara's (2005) framework that suggests impulsive 
behaviour can occur in three interacting, but potentially dissociable cognitive domains: 1) decision-making, 2) perceptual impulse control, 3) motor impulse control. Cognitive assessment tasks are designed to isolate which specific cognitive processes pertaining to each broad domain are affected, and are thus likely to cause impulsive behaviour.

\subsubsection{Decision-making definitions of impulsivity}

\subsection{Reward sensitivity}

Some decision-making definitions of impulsivity suggest that impulsive people are overly sensitive to reward which biases choices toward smaller-sooner over largerlater reward (e.g., Bechara, 2007; Monterosso \& Aimslie, 1999). Reward sensitivity is central to Gray's Reinforcement Sensitivity Theory (Gray, 1987). Gray suggested that all behaviour is motivated by two systems: the Behavioural Activation System (BAS) and Behavioural Inhibition System (BIS). The BAS is sensitive to reward, activating approach behaviour in response to positive incentives. The BIS is sensitive to punishment, activating inhibition of behaviour when environmental cues indicate that a negative consequence would follow if the approach behaviour were enacted (Colder \& O’Connor, 2004; Gray, 1987). Gray suggested that differences between BAS and BIS functioning are reflected in the personality dimensions of impulsivity and anxiety, respectively. Impulsive people are thought to have a strong BAS, which leads to dominant approach behaviour toward reward, such as preferring instant gratification above delayed reward. Related to this account are definitions of impulsivity such as failure to delay gratification and delay-aversion (e.g., Holt, Green \& Myerson, 2003; Solanto et al., 2001). 
Reward-sensitivity is assessed via cognitive tasks that utilise delay of gratification paradigms, or forced choice between smaller-sooner and larger-later rewards. The sooner a path to delayed reward is abandoned, the more impulsive decision-making is considered to be (Kirby \& Finch, 2010; Reynolds, Richards, Horn \& Karraker, 2004). For example, in the Two-choice Delayed Reward task (TC; Dougherty et al., 2003) and the Single-Key Impulsivity Paradigm (SKIP; Dougherty, Mathias, Marsh \& Jagar, 2005), subjects must indicate their preference between a hypothetical choice, such as receiving $\$ 10$ in 30 days or \$2 now. Faster rates of discounting delayed reward have been demonstrated in clinical populations such as drug users, pathological gamblers and patients with frontal lobe damage (Bechara, 2005; Reynolds, 2006).

\subsection{Response perseveration}

In contrast to an overactive Behavioural Activation System causing hypersensivity to reward, impulsivity is also operationalised as a weak Behavioural Inhibiton System (BIS; Gray, 1987). Consequently, impulsive people experience less anxiety at the prospect of punishment relative to people with a stronger BIS (Gray, 1987). From this perspective, once impulsive people have developed a strong — particularly reward-related—response set, they are less likely to inhibit dominant responses even when reinforcement contingencies change and the dominant response set is no longer correct (Fowles, 1980). According to this account, impulsivity is defined as response perseveration, or a failure to inhibit behaviour that is no longer rewarded and likely to be punished (Newman, Patterson \& Kosson, 1987).

Cognitive assessment tasks based on response perseveration operationalisations of impulsivity build and reward a dominant response set, then change reinforcement 
contingencies such that continuing the dominant response set becomes punished. For example, on the Wisconsin Card Sorting Test (Heaton, 1981; Heaton et al., 1993), subjects are presented with cards displaying different shapes that vary in colour and number. Subjects are instructed to sort the cards but they are not explicitly told which dimension to sort the cards on, only whether their sorting strategy is correct or incorrect. Once the correct sorting rule is achieved, the sorting rule changes. Impulsive or perseverative responding is expressed if subjects continue to use a sorting rule that was previously rewarded, but is now incorrect due to a rule change. Similarly, in Newman's Card Playing Test (Newman, Patterson \& Kosson, 1987), subjects can choose between continuously playing cards that return wins and losses, or quitting the game. As the number of cards played increases, the probability of choosing cards that return losses increases. Therefore, continuing to make card selections despite repeated punishment is indicative of impulsive, or perseverative responding.

Using a computerised task similar to Newman's card test with a steadily increasing punishment-to-reward ratio, O'Brien and Frick (1996) found that children with severe conduct problems and no anxiety disorder played more trials than children with conduct problems and a co-morbid anxiety disorder, non-anxious children with ADHD, and children in the normal control group. Consistent with Gray's BIS theory, this result supports the importance of anxiety in learning from punishment, as well as the role of reward dominance and perseverative responding in understanding antisocial behaviour. Adults with antisocial personalities - particularly psychopathy — are also found to display passive avoidance deficits especially when inhibition of a previously rewarded response must be inhibited to avoid punishment (Newman, Widom \& Nathan, 1985). 
This deficit is thought to arise from a lack of conditioned anxiety that punishment normally evokes (Lykken, 1995).

The Iowa Gambling Task also assesses the ability to learn reward and punishment contingencies (Bechara, 2007). Subjects are presented with four decks of cards (A,B,C,D) and told to select cards one at a time from any deck they wish. Card selections are accompanied by winning an amount of money, but unpredictably some trials are also accompanied by a loss. Each deck differs on the basis of its reward and punishment contingencies: Decks A and B have high rewards, but even higher losses; whilst Decks C and D have lower rewards, but even lower losses. Therefore, continued selections from Decks A and B results in an overall net loss, whilst continued selections from Decks C and $\mathrm{D}$ results in an overall net win. Advantageous decision-making is thus expressed by a greater number of choices from Decks C and D relative to Decks A and B. After an initial period of experiencing the rewards and punishments of each deck, neurologically healthy people typically learn to avoid the bad decks (A, B) and choose from the good decks (C, D; Bechara, 2007). However, groups of people who make disadvantageous real-world choices (e.g., patients with frontal damage, substance abusers, psychopaths, people with Attention Deficit/Hyperactivity Disorder) tend to continue making disadvantageous card selections and appear unable to learn the advantageous strategy (see Buelow \& Suhr, 2009 for a review).

Although different to Bechara's criteria for optimal decision-making on the gambling task, recent research has emerged to show that healthy decision-making can also be conceptualised as loss aversion. In addition to differences in reward and punishment magnitude, the card decks in the Iowa Gambling Task also differ in reward 
and punishment frequency. By comparing deck preferences according to both magnitude and frequency of reward and punishment, researchers have shown that neurologically healthy participants tend to prefer decks with low loss frequency (B and D) over and above decks with the largest net reward (C and D; e.g., Steingroever, Wetzels, Horstmann, Neumann \& Wagenmakers, 2013). Thus, researchers argue that the ability to consider longer-term outcomes is not the only way to conceptualise healthy decisionmaking (Steingroever, Wetzels, Horstmann, Neumann \& Wagenmakers, 2013).

\subsubsection{Perceptual impulse control definitions of impulsivity}

Before behavioural responses are made, multiple action plans or response options are automatically generated. Choosing options that are consistent with goals or personal standards, and inhibiting the urge to act on options that are inconsistent with goals requires perceptual impulse control skills. Perceptual impulse control is a broad term, which includes the ability to focus on some thoughts and block out interference from other thoughts, and the ability to flexibly shift attention between multiple thoughts at once (Bechara, Noel \& Crone, 2006). Put another way, this type of impulse control refers to controlling thoughts - prioritising some and inhibiting others — before thoughts are translated to behaviour. Accordingly, perceptual impulse control-related definitions of impulsivity describe impulsivity as a rigid cognitive style (Shapiro, 1981), cognitive inflexibility (e.g., Bechara, 2007), and poor ability to resist cognitive interference (Robbins, Gillan, Smith, de Wit \& Ersch, 2012).

Cognitive assessment tasks that require resistance to interference from habitual thoughts, and flexible attention shifting between perceptual sets are used to capture perceptual impulse ability (Bechara, Noel \& Crone 2006). Because impulsive people find 
it particularly difficult to respond quickly and accurately when flexible cognition is required, impulsive performance is expressed by elevated error rates and longer response times for conditions that require inhibition and set shifting, compared to easier conditions that do not require such higher-order skills. Well-known cognitive tasks based on this conceptualisation include Stroop tests (Stroop, 1935), Trail Making Tests (Lezak, 1995; Reynolds, 2002), and the Intra-dimensional/Extra-dimensional shift task (e.g., Downes et al., 1989). For example, in the classic Stroop test, subjects are presented with a colour word (e.g., green) printed in an incongruent ink colour (e.g., red; Stroop, 1935). Subjects must inhibit their over-learned response to read the word and instead name the ink colour. As another example, the Comprehensive Trail-Making Test (Reynolds, 2002) requires subjects to flexibly shift attention between perceptual sets, alternately joining consecutive numbers with consecutive letters of the alphabet (e.g., 1-A-2-B-3-C). Impairments in the ability to resist cognitive interference and in cognitive flexibility have been observed in clinical populations that typically behave impulsively in naturalistic settings, including individuals with substance dependence (Verdejo-Garcia, Bechara, Recknor \& PerezGarcia 2006), Anorexia and Bulimia Nervosa (Tchanturia et al, 2004) and Bipolar Disorder (Shamay-Tsoory, Harari, Szepsenwol \& Levkovitz, 2009).

\subsubsection{Motor impulse control definitions of impulsivity}

Much of human behaviour is generated automatically by environmental stimuli that trigger bottom-up responses. To live adaptively, we must override automatic behaviours when they are contrary to our goals. The ability to override automatic responses is called inhibitory control, or motor impulse control: the capacity to stop actual behaviour when necessary (Logan, Schachar \& Tannock, 1997). When inhibitory 
control fails, impulsive behaviour is expressed. Thus, motor impulsivity is typically defined as response disinhibition or inhibitory dyscontrol (Cherek, Moeller, Dougherty \& Rhoades, 1997; Logan, Schachar \& Tannock, 1997).

Cognitive assessment tasks of inhibitory control build a dominant motor response and periodically force inhibition of that response. Well-known examples include Continuous Performance Tests (CPTs) and Stop Signal or Go/No-go discrimination tasks. CPTs present subjects with a string of rapidly presented stimuli, and subjects must selectively respond to target stimuli and inhibit responses to non-target stimuli. Responding quickly to non-target stimuli (i.e., commission errors) is indicative of impulsivity (Dougherty, Marsh \& Mathias, 2002). Some well known CPTs include the Immediate and Delayed Memory Task (IMT/DMT; Dougherty, Marsh \& Mathias, 2002), Conner's CPT (Conners, 2002), the Integrated (or intermediate) Visual and Auditory CPT (IVA, Sandford \& Turner, 1995), the Test of Variables of Attention: Visual (TOVA-V) and Auditory (TOVA-A; Greenberg, 1998, 2002; Greenburg \& Dupuy, 2000, 2001).

Based on correlational studies, a strong relationship between CPT performance, direct behavioural observations, outcomes on other measures of attention and executive control, and behavioural ratings suggests that CPTs have moderate to high ecological validity (Riccio, Reynolds \& Lowe, 2001). Elevated commission errors are found for people known to behave impulsively in naturalistic settings, including individuals diagnosed with Conduct Disorder (Dougherty, Bjork, Marsh, \& Moeller, 2000), Borderline Personality Disorder (Dougherty, Bjork, Huckabee, Moeller, \& Swann, 1999), Bipolar Disorder (Swann, Anderson, Dougherty, \& Moeller, 2001), Attention Deficit 
Hyperactivity Disorder (Halperin, Wolf, Greenblatt, \& Young, 1991), and disruptive behaviour disorders (Bjork et al., 2000).

Stop Signal tasks assess the speed of the stopping process, by simulating a race between two conflicting response sets: an active response that is set in motion by an initial 'go' signal, and a conflicting inhibitory response that is set in motion by an unexpected 'stop' signal (typically on 20-30\% of trials; Crean, deWit, \& Richards, 2000; Logan \& Cowan, 1984). The outcome on any given stop trial is thought to reflect the relative speeds of the 'go' reaction and the stopping or inhibitory process (Logan \& Cowan, 1984; Solanto et al., 2001). Normal inhibitory control is indicated by a fast 'go' and stop process; that is, fast and accurate responding. In contrast, impulsivity is expressed when the stop process takes longer to reach completion than the 'go' process (Verburggen, Logan \& Stevens, 2008). Studies have shown that children with ADHD need as much as 70 milliseconds longer than age-matched controls to process stop cues and successfully inhibit responses (Schachar, Mota, Logan, Tannock, \& Klim, 2000). Elevations in time needed to stop a response have also been observed for individuals with Obsessive-Compulsive Disorder and Trichotillomania (Chamberlain, Fineberg, Blackwell, Robbins \& Sahakian, 2006), individuals who use marijuana (McDonald, Schleifer, Richards, \& de Wit, 2003), chronic cocaine abusers (Fillmore \& Rush, 2002; Kaufman, Ross, Stein \& Garavan, 2003; Li, Milivojevic, Kemp, Hong, \& Sinha, 2006), ecstasy users (Moeller et al, 2002), young adults with ADHD (Barkley, Fischer, Smallish \& Fletcher, 2002), and older adults with ADHD (Bekker et al., 2005; Epstein, Johnson, Varia \& Conners, 2001). 


\subsubsection{Defining and assessing impulsivity: summary}

The diverse range of impulsivity definitions and assessment methods adopted in clinical and forensic literatures is testament to the complexity of the impulsivity construct. Each type of assessment method plays a crucial role in capturing this complexity. Self-report questionnaires allow assessors to capture multiple dimensions of impulsivity, whilst behaviourally based measures allow assessors to establish how people typically behave across time and in different contexts. Importantly, the design of cognitive assessment methods allows assessors to isolate impairment in specific cognitive processes that give rise to impulsive behaviour. As I will outline in the following chapter, the ability to isolate which cognitive domains — and processes within those domains - are impaired in antisocial samples is important for understanding criminal risk, particularly regarding developmental theories of antisocial behaviour across the lifespan. 


\section{Chapter 2}

\section{Impulsivity and antisocial behaviour across the lifespan}

\subsection{Background}

For the most part, studies that have assessed impulsivity from behavioural and personality approaches find strong relations between impulsivity and antisocial behaviour. In contrast, research that draws on the cognitive approach is less conclusive, particularly regarding the developmental trajectory of cognitive mechanisms of impulse control in adulthood. The present chapter reviews the literature illustrating associations between impulsivity and antisocial behaviour at different developmental phases in the lifespan. In addition, the chapter highlights the necessity of assessing impulsivity from the cognitive approach in adult antisocial populations, to clarify how cognitive impulse control mechanisms contribute to criminal risk in adulthood.

\subsection{The developmental relationship between impulsivity and antisocial risk}

Poor impulse control is normative at some stages in the life course; toddlers break rules and throw tantrums because they have not yet learned any better. However, if impaired impulse control persists at chronic levels across the life course, risk of antisocial behaviour increases (Andrews \& Bonta, 2006). Studies investigating the developmental trajectories of antisocial behaviour suggest that variation in impulse control can be genetically inherited or acquired from pre or post-natal disruptions to normal neuropsychological development (Glaser, 2000; Raine, Brennan, Mednick \& Mednick, 1996). In her influential theory, Moffitt (1993) described two hypothetical groups of 
offenders who differ in their underlying neuropsychological capacity for impulse control and in their socialisation experiences. Neuropsychological and socialisation differences make important contributions to the trajectories of each group's offending careers (see Moffitt, 1993 for a full description).

\subsubsection{Moffitt's developmental taxonomy}

According to Moffitt's (1993) developmental taxonomy, antisocial behaviour can be near normative for one group. People in this group have good neuropsychological health and intact cognitive functioning. Furthermore, they develop in contexts characterised by positive socialisation experiences that deliver clear reinforcement contingencies such that good behaviour is rewarded and bad behaviour is punished. However, despite intact cognitive functioning and positive socialisation experiences, people in this group will deliberately engage in antisocial behaviour during adolescence to meet unique developmental needs during this period, such as providing a way to assert independence (Moffitt, 1993) and providing a way to impress peers (Gardner \& Steinberg, 2005). But eventually the costs of behaving antisocially become too high and other, non-costly alternatives are sought. Because people in this group develop in the context of positive socialisation experiences, they have a prosocial behavioural repertoire to return to. Furthermore, because their fundamental cognitive abilities are intact, they have the requisite skills to inhibit antisocial behaviours and return to prosocial means of meeting developmental needs. Moffitt (1993) referred to this normative group as adolescence-limited (AL) offenders; a group characterised by criminal careers that begin and end in adolescence or early adulthood (Piquero, Diamond, Jennings \& Reingle, 2013). 
By contrast, Moffitt asserted that a much smaller group of people displays a nonnormative, dysfunctional pattern of antisocial behaviour that begins in childhood and continues throughout adulthood. This group is called Life-course-persistent (LCP) offenders. In the LCP group, the interaction between inherited or acquired neuropsychological deficits and a disadvantaged developmental environment underlies increased life-long risk for antisocial behaviour (Moffitt, 1993). Disruptions to normal neuropsychological development and poor socialisation experiences determine that children on the LCP path typically have difficult temperaments which remain relatively stable over the life course but can be modified by experience (Delisi \& Vaughan, 2011; Goldsmith et al, 1987; Moffitt, 1993; Rothbart, Ahadi \& Evans 2000). Two components of difficult temperament particularly important for understanding criminal risk include disinhibition (inhibitory deficits, sensation-seeking, activity level) and negative emotionality (including anger and irritability; Colder \& Stice, 1998; Moffitt, 1993). Although Moffitt did not elaborate on the specifics of her operational definition of disinhibition, other authors have described disinhibition in personality terms as the "nexus of impulsivity and negative affectivity" (Patrick, Fowles \& Krueger, 2009, p.925).

Difficult temperament affects how children interact with their environment (Moffitt, Lynam \& Silva, 1994). Given the hereditary basis to neuropsychological health and temperament, children on the LCP path are likely to be born to parents with similarly difficult temperaments (Moffitt, 1993). Thus, problematic behaviour caused by children's difficult temperament is more likely to be met by parental responses that serve to exacerbate problem behaviour (Gottfredson \& Hirschi, 1990; Moffitt, 1993). 
Inhibitory deficits and poorly regulated behaviour can affect risk of antisociality both directly and indirectly (White et al., 1994). Arguably, impaired cognitive capacity to consider consequences of actions and inhibit antisocial behaviour will directly increase the likelihood that antisocial behaviour will occur. Indirectly, involvement in antisocial behaviour limits opportunities to access the types of prosocial socialisation experiences that foster the healthy development of impulse control (e.g., early school leaving restricts exposure to discipline from social institutions; Moffitt, Caspi, Harrington \& Milne, 2002). Unlike adolescence-limited offenders, offenders on the LCP path are less likely to recover from antisocial behaviour: "continuity of offending occurs because the relative ordering of individuals on the interaction between neuropsychological risk and disadvantaged environment stays fairly consistent over time" (Piquero \& Moffitt, 2011, p.60).

\subsubsection{Summary of Moffitt's developmental taxonomy}

Moffitt's LCP theory provides the theoretical basis for the prediction that offenders who remain chronically antisocial in adulthood are likely to have underlying cognitive impulse control impairments. Cognitive impairment manifests temperamentally in impulsive personality and behaviour. Studies to date show that children and adolescents, who exhibit poorer performance on cognitive tests of impulsivity, and higher scores on personality or behavioural ratings of impulsivity measures, also tend to be antisocial. However, as will be outlined later, the picture is less straightforward in adulthood. 


\subsubsection{Empirical support for the LCP theory during childhood and adolescence}

A number of studies empirically support the postulates of the LCP theory in antisocial children and adolescents (e.g., Gibson \& Tibbetts, 2000; Kratzer \& Hodgins, 1999; Piquero, 2001; Piquero \& Brezina, 2001; Piquero \& Tibbetts, 1999; Tibbetts \& Piquero, 1999). In particular, findings from the Dunedin Multidisciplinary Health and Development Study (see Silva, 1990 for a description) provide cross-sectional and longitudinal support for developmental differences between individuals on AL and LCP paths.

In an early follow-up, Moffitt and colleagues found that children displaying early antisocial behaviour (i.e., before 3 years of age) also had difficult temperaments, as rated by parents and a third-person observer (Moffitt, Caspi, Dickson, Silva \& Stanton, 1996). When the same children were re-assessed at age 18 , they described themselves as enjoying an: "impulsive, impetuous lifestyle rather than a reflective, planful one" (Moffitt, Caspi, Dickson, Silva \& Stanton, 1996, p.420). In a separate study, Henry, Caspi, Moffitt and Silva (1996) tested whether children's behavioural control at 3 and 5 years of age was related to official convictions at age 18. Poor behavioural control was operationalised as "an inability to modulate impulsive expression, impersistence in problem solving and sensitivity to stress and challenge that is expressed in affectively charged negative reactions" (p.59). Poor behavioural control as a child was higher in those with at least one violent conviction at age 18 .

In a different sample, using a battery of neuropsychological measures adapted for use with preschool-aged children (approximately 4 years), Raaijmakers et al. (2008) found that deficits on one factor—inhibition—differentiated aggressive preschoolers 
from non-aggressive children. Furthermore, the association between inhibitory deficits and aggression was maintained after controlling for attention problems. In addition to explaining unique variance beyond other domain-general cognitive abilities like attention, performance on cognitive tasks and behavioural ratings of impulsivity by parents and teachers also accounted for variance in delinquency over and above IQ and social-level factors in young boys (White et al., 1994). Specifically, scores indicative of a disinhibited, under-controlled child who displayed motor restlessness and an impatient, impersistent approach to completing cognitive tasks added the most variance to delinquency scores over and above IQ and SES at age 10, and to serious, stable delinquency at 12-13 years of age (White et al., 1994). However, a more recent longitudinal study linking cognitive impulsivity at 12 years and offending up until 28 years shows that cognitive impulsivity did not independently influence offending at any developmental stage for boys with low IQ (Loeber et al., 2012).

In support of the LCP postulates in adolescence, Moffitt, Lynam and Silva (1994) showed that a boy's poor neuropsychological functioning at age 13 , including cognitive inflexibility, was related to a higher number of crimes committed by age 18 . Similarly, in a sample of early-onset (< age 12), late-onset (> age 13) offenders, and non-offending male youths (average age of 15 years in all groups), juveniles who displayed the most rapid cognitive tempo, the poorest inhibitory control and the highest self-report impulsivity scores were more likely to be early-onset delinquents (Carroll, Hemingway, Bower, Ashman, Houghton \& Durkin, 2006).

Greater impulsivity is also associated with higher rates of offending in juvenile populations. Using an earlier version of Eysenck's $I_{7}$ that distinguishes between 
impulsiveness as a failure to consider the risks of behaviour, and venturesomeness as a preference for risky activities, Eysenck and McGurk (1980) and Thornton (1985) found that delinquents (14-16 years) from multiple detention centres self-reported significantly higher impulsivity (but not venturesomeness) than non-delinquent, young adult controls. Failure to consider the risk associated with certain behaviours in the delinquent samples was significantly, moderately and positively correlated with self-reported delinquency, including acts of vandalism, aggression, weapons possession and theft.

In a study with both cross-sectional and longitudinal components, Luengo, Carrillo-de-la-Pena, Otero and Romero (1994) examined the association between selfreported impulsive personality on Eysenck's $I_{6}$ and Barratt's BIS-10, and self-reported adolescent antisocial behaviour. In a non-institutionalised sample of adolescents aged 12-18 years, cross-sectional analyses showed that all total and sub-scale impulsivity scores were significantly, positively correlated with all antisocial outcomes including self-reported vandalism, theft, aggression, rule-breaking, drug abuse and total antisocial scores. Specifically, scores on the BIS-10 non-planning factor were most strongly related to antisocial behaviour overall, highlighting the tendency for delinquents to be presentfocused, with no regard for the longer-term consequences of their actions. Longitudinal analysis sought to determine whether impulsivity was related to stability or change in delinquency or non-delinquency over a 1-year period. Whilst nearly half of the subjects remained non-delinquent from time 1 to time 2, delinquents that increased their involvement in antisocial behaviour during the follow-up period were differentiated from stable non-delinquents by higher $\mathrm{I}_{6}$ impulsivity scores and higher scores on Barratt's non-planning and motor, but not attentional impulsivity, at both time 1 and time 2 . 
Taken together, Luengo and colleagues demonstrated that impulsive personality was not only associated with antisocial behaviour at one point in time, but that impulsive personality was associated with future increases in antisocial behaviour. The fact that the attentional component of impulsive personality did not explain variance in antisocial behaviour in their study highlights the level of specificity necessary to establish how impulsive personality contributes to criminal risk; for individual offenders or different sub-groups of offenders, some dimensions of impulsive personality might explain significant variance, whilst other dimensions might not. The same specificity applies to understanding the contribution of cognitive mechanisms of impulse control to criminal risk, given the number of studies that found differential patterns of impairment as a function of age of offending onset, type and severity of offending history.

\subsection{Summary of child and adolescent studies}

Many studies from behavioural, personality, and cognitive approaches to assessing impulsivity illustrate cross-sectional, retrospective and longitudinal relationships between impulsivity and antisocial behaviour in childhood and adolescence. These studies also illustrate how specific patterns of impulsive personality and impairment in cognitive impulse control mechanisms differentially apply to offender subgroups based on individual-level factors. Such specificity suggests that the contribution of impulsivity to risk of antisocial behaviour early in life is robust but complex; this picture becomes even more complex and relatively less robust in adulthood.

\subsubsection{Empirical support for the LCP theory during adulthood}

Babinksi, Hartsough and Lambert (1999) found support for predictive links between early impulsivity and antisocial behaviour in adulthood. The authors obtained 
behavioural ratings from parents and teachers of inattention, hyperactivity-impulsivity and conduct problems in a sample of children identified as hyperactive at approximately 9 years of age. At 26 years, the same participants were interviewed about their involvement in crime as an adult and official records of arrests were obtained. Babinski and colleagues found that symptoms of hyperactivity-impulsivity—but not inattention as a child — and conduct disorder problems during childhood predicted self-reported criminal involvement and official arrests as an adult.

However, findings from other major developmental investigations challenge the consistency of the relationship between childhood or adolescent impulsivity and antisocial behaviour in adulthood. For example, in a longitudinal survey of males followed from the age of 8 to 32 years, Farrington (1989) examined a range of potential predictors and correlates of aggression and violence. In addition to adverse family and social factors, high impulsivity scores on psychomotor tests during childhood (8-10 years) predicted aggression during adolescence ( $12-14$ years); impulsive boys were described by their teachers as "disobedient, difficult to discipline, unduly rough during playtime, quarrelsome and aggressive, over competitive with other children and unduly resentful of criticism or punishment" (Farrington, 1989, p.83). However, high impulsivity during childhood did not significantly predict violence in adulthood (32 years).

In the 26-year follow-up of participants from the Dunedin Multidisciplinary Health and Development Study, contrary to expectation, LCP offenders had lower impulsive personality than their AL counterparts, as indicated by self-report and informant ratings (Moffitt, Caspi, Harrington, \& Milne, 2002). Despite being less impulsive than AL offenders, self-report and informant ratings confirmed that LCP 
offenders were higher risk than AL men in many areas of life including: more frequent, varied and serious offending and official convictions, poorer mental health (including markers for Antisocial Personality Disorder) ${ }^{1}$, greater substance use, more social isolation, more trouble in personal and family relationships (e.g., more controlling towards romantic partner, more likely to hit a child and father several children), poorer education and economic outcomes, and greater unemployment rates (Moffitt, Caspi, Harrington, \& Milne, 2002).

Three issues hamper the interpretation of results that contradict predictions of Moffitt's LCP theory. First, investigations like Farrington's (1989) study that have used childhood impulsivity to predict adult antisocial outcomes cannot inform what happens to impulse control by adulthood. Thus, it is unclear why childhood impulsivity no longer seems to account for criminal behaviour in adulthood. Second, studies like Moffitt and colleagues' follow-up that have re-assessed impulsivity in adulthood tend to re-assess impulsivity using personality and behavioural, but not cognitive, measures. As will be outlined in more detail below, personality and behavioural measures are limited in their capacity to provide reliable indications of underlying cognition.

Third, findings from studies that have assessed cognitive mechanisms of impulse control in adult offender samples are inconsistent. Previous studies have not typically assessed or reported whether offenders were on the LCP path or not; therefore, it is difficult to evaluate cognitive findings in terms of whether they support or refute Moffitt's LCP prediction of underlying cognitive impairment in impulse control. Generally, similar to the child and adolescent studies, relationships between cognitive

\footnotetext{
${ }^{1}$ Informant ratings showed significant LCP/AL differences in psychopathology but selfreport ratings did not differ.
} 
mechanisms of impulse control and antisocial behaviour tend to depend on individuallevel and criminal history factors (e.g., history of violent versus non-violent offending). Thus, it remains unclear how cognitive mechanisms of impulse control contribute to criminal risk in adulthood. A brief review of findings from adult offender studies investigating impulsivity from the cognitive approach follows.

\subsubsection{Cognitive impulse control in adult offenders}

From the perspective of impulsivity as decisions driven by reward sensitivity, Cherek, Moeller, Dougherty and Rhoades (1997) found that male parolees with a history of violence demonstrated more reward-sensitivity than parolees without a history of violence. However, non-violent parolees were also more reward-sensitive than nonoffender controls: reward-sensitivity was relatively more pronounced in violent offenders but offenders overall were more reward-sensitive than the general population. Cherek and Lane (1999) later validated the differential relationship between violence history and impulsivity in an identical study with female parolees.

Yechiam and colleagues (2008) also assessed decision-making in a sample of male and female offenders incarcerated for theft, robbery, driving, sexual, drug and serious violence index offences. Yechiam and colleagues sought to disentangle causal explanations for impulsive, reward-sensitive decision-making by differentiating: a tendency to attend to gains and pay less attention to losses, a tendency to focus on recent outcomes and rapidly discount past outcomes, or a tendency to choose randomly. All offender groups chose smaller-sooner rewards over choices that would provide greater reward in the long-term significantly more than non-offender controls. However, most offenders with driving, drug, sexual, or theft offences preferred smaller-sooner reward 
because they tended to pay high attention to gains, whereas offenders with robbery and assault/murder offences preferred smaller-sooner reward because they weighted recent versus past outcomes more heavily and displayed more random responding when making decisions. This study highlights important variation in the unique causal mechanisms underlying what appears to be the same impulsive decision-making style.

In relation to perceptual impulse control processes, Bergvall, Wessely, Forsmen and Hansen (2001) administered a battery of cognitive tests to adult violent offenders, persons with mild mental retardation and non-offenders. Collectively, the test battery assessed spatial and figurative working memory, planning and attentional set shifting. Although violent offenders had normal intellect and performed comparably to the control groups on tests of working memory and planning, they committed three times more errors as the controls on attentional set shifting and displayed substantial difficulty in reversing stimulus-reward associations. Consistent with Bergvall and colleagues, Hancock, Tapscott and Hoaken (2010) found that inhibition and set shifting deficits in a sample of adult incarcerated offenders significantly predicted the frequency and severity of previous violence, but not non-violent offending. Similarly, in a New Zealand prison sample, Crump (2005) found that offenders had the most difficulty with inhibition and cognitive flexibility relative to other executive functions, such as planning. Lastly, in a male sample with Antisocial Personality Disorder (ASPD) who also displayed traits of psychopathic personality, Dinn and Harris (2000) found that ASPD, psychopathic subjects experienced more cognitive interference on the Stroop test relative to males recruited from the community, despite superior performance to normative comparisons on a divergent thinking test. 
Regarding motor impulse control, a number of studies have found no abnormality in the 'go' process but isolated impairment in the speed or accuracy of the stopping process. Compared to cognitively intact subjects, elevated commission error rates have been observed in people with ASPD (e.g., Barkataki, Kumari, Das, Sumich, Taylor \& Sharma., 2008; Dolan \& Park, 2002), psychopathy (e.g., Lapierre, Claude, Braun \& Hodgins, 1995), and hospitalised adult violent offenders (Enticott, Ogloff, Bradshaw \& Daffern, 2007). Interestingly, the hospitalised violent offenders in Enticott and colleagues' (2007) study still performed within what is considered a normal range for the specific motor impulse control outcome used to evaluate performance. Therefore, despite sub-optimal performance relative to the general population, it is unclear exactly how impaired response inhibition must be before it is dysfunctional enough to contribute to criminal risk. It is also unclear who stable response inhibition impairments are: Chen and colleagues observed inhibitory impairment in a violent offender sample, but only when time constraints were placed on responding to prevent strategic control of error rates; without a time constraint, response inhibition was normal (Chen, Muggleton, Hung-Juan, Tzeng \& Hung, 2008).

Some studies have found no impairments in adult offender populations expected to perform poorly. For example, some researchers have found that psychopathic offenders perform comparably to normative populations of similar age, education and IQ on tests of cognitive impulse control (e.g., Hart, Forth \& Hare, 1990; Pham, Vanderstukken, Philippot \& Vanderlinden, 2003).

Other studies show that offender sub-groups have dissociable impairments between cognitive impulse control domains. For example, Swann and colleagues (2011) 
recruited individuals diagnosed with Bipolar Disorder and symptoms of ASPD, with and without a history of criminal convictions. Individuals with Bipolar Disorder, ASPD symptoms and a conviction history displayed impaired response inhibition but they were not impaired in their ability to wait for reward, compared to individuals with Bipolar Disorder, ASPD symptoms and no criminal history. In direct contrast, Mitchell, Colledge, Leonard and Blair (2002) found that adult psychopaths were particularly reward sensitive in a decision-making task and displayed deficits in response reversal (i.e., response perseveration) whilst set shifting (i.e., perceptual impulse control) was intact.

\subsection{Summary of cognitive impulse control in adult offenders}

The relationship between cognitive impulse control ability and antisocial behaviour in adult offenders is not straightforward. Studies to date vary in their chosen methodologies for assessing cognitive impulse control. Often, the selection of specific assessment tasks does not appear to be guided by theory (Nigg, 2000). Studies also differ in the normative groups offender performance is compared against; some studies evaluate offender performance against non-offender populations, whilst others evaluate performance between offender sub-groups that differ in theoretically meaningful ways (e.g., violent versus non-violent offending history). Given the inconsistencies to date in studies assessing cognitive mechanisms of impulse control in adult offenders, it remains unclear whether cognitive impairments maintain antisocial risk in adulthood. Studies that assess impulsivity in adult offender populations using personality and behavioural measures cannot provide sufficient evidence of underlying cognitive impulse control functions. In order to understand whether cognitive deficits maintain LCP risk for antisocial behaviour, cognition must be more reliably assessed in adulthood via cognitive 
assessment methods. I now turn to a more detailed discussion of the specific advantages of adopting the cognitive approach to assessing impulsivity.

\subsection{Advantages of the cognitive approach}

The major advantage of cognitive assessment methods concerns experimental control. Personality and behavioural assessments rely on the inference of cognitive processes from naturalistic behaviour that is not experimentally controlled. Whilst naturalistic behaviour is no doubt the most ecologically valid behaviour, the ability to isolate impairment in the specific cognitive processes causing behaviour is limited. Without experimental control, the range of cognitive processes that could explain impulsive behaviour is limited to guesswork. In the context of treatment interventions to reduce criminal risk, reliable methods for assessing causal mechanisms of impulsive behaviour is important: the development, implementation and evaluation of effective interventions for dysfunctional impulsive behaviour are difficult to achieve if the problem is ill defined and poorly understood (Eaves, Tien \& Wilson, 1997).

The limitations of inferring cognition from personality and behavioural outcomes is highlighted by a large body of research showing low and non-significant correlations between outcomes from personality and cognitive impulsivity assessments (e.g., Cyders \& Coskunpinar, 2012; Enticott, Ogloff, Bradshaw \& Fitzgerald, 2008; Glicksohn, Leshem, \& Aharoni, 2006; Parker \& Bagby, 1997; Reynolds, Ortengren, Richards \& de Wit, 2006). For example, whilst some correlational studies in cognitively intact populations show that impulsive personality is associated with poorer inhibition on stop signal tasks (e.g., Gorlyn Keilp, Tryon \& Mann 2005; Logan, Schachar \& Tannock, 1997), others fail to support a relationship between impulsive personality, time to 
inhibition (e.g., Cheung, Mitsis, \& Halperin, 2004; Lijffijt et al., 2004), and accuracy of inhibition (e.g., Fallgatter \& Herrmann, 2001; Horn, Dolan, Elliott, Deakin \& Woodruff, 2003).

There are several competing explanations for this incompatibility. First, incompatibility could be explained by variation in measurement modality. Self-report personality measures are designed to capture the sum of impulsive cognition at an aggregate level, whereas cognitive measures are designed to isolate specific cognitive processes that contribute to impulsive behaviour. Because each measurement modality is intentionally designed to capture impulsivity in different ways, it seems reasonable to expect that outcomes on each type of measure would not completely overlap. However if, as Moffitt suggests, impulsive personality is the culmination of impairments in the cognitive processes responsible for impulse control, we would expect more overlap in outcome scores than is typically observed, regardless of measurement modality.

Second, incompatibility could be explained by the measurement error introduced by unavoidable response biases inherent to self-report methods. Self-report impulsivity questionnaires rely on people's explicit insight into how they typically think and act, whereas cognitive assessments implicitly test skills, thus bypassing reliance on subjective insight. Previous research shows that it is common for outcomes from implicit and explicit measures to be weakly related, and even unrelated (e.g., Karpinski \& Hilton, 2001). The limitations of asking someone to judge their own impulsive cognitive processes (e.g., "I tend to act before I think") are illustrated by the following analogy: consider trying to describe all of the physical processes that underlie our ability to breathe. Although knowledge about the outcome of those processes-breathing 
behaviour - is consciously available (e.g., the chest rises and falls, breathing pace might change in response to physical activities), the physical processes governing that behaviour are so automatic that the accuracy with which you could detail them would be limited due to lack of insight. Not only is impulsive behaviour considered an automatic process that unfolds outside of awareness (Strack \& Deutsch, 2004), one theorised cause of impulsive behaviour is a lack of reflection (e.g., Bechara, 2007). Arguably then, it is counterintuitive to expect any impulsive individual — criminal or otherwise — to have insight into why they behave impulsively, because impulsive behaviour occurs automatically without awareness. Put another way, the very trait being assessed is the very trait that is likely to cause inaccuracies in self-report responses: a lack of reflection.

A third possibility is that impulsive personality and cognition are not as closely linked as Moffitt's (1993) LCP theory proposed. Such an explanation makes intuitive sense given that personality impulsivity captures factors such as personal preference for spontaneity, excitement and desire to satisfy immediate needs. On the other hand, cognitive mechanisms of impulse control relate to the fundamental cognitive processes that support the regulation of impulses. Is a preference for an impulsive lifestyle necessarily caused by deficits in the cognitive process that support impulse control? Unexplained variance between personality and cognitive impulsivity raises the possibility that there are different types of impulsivity that might differentially contribute to criminal risk.

Specifically, impulsive-looking behaviour need not be caused by cognitive failures. Without assessing cognition proximal to behaviour, we cannot rule out whether someone was well aware of the consequences but deliberately chose to take the risk. In 
this sense, the cause of behaviour would be better explained by venturesomeness or sensation seeking, not impulsivity (Evenden, 1999). Whilst Moffitt's (1993) LCP theory highlights sensation seeking as one component of difficult temperament, it does not appear to be considered interchangeable with the disinhibition component. Furthermore, it is typically agreed that impulsive explanations for behaviour are dissociable from risktaking explanations (e.g., Miller, 1992).

Brunner and Hen (1997) illustrate how the same behavioural response can be reached via very different cognitive routes. Consider an individual who knows the possibility of earning one of two presented rewards: a smaller immediately available reward, or a greater delayed reward. The individual might choose the small immediate reward because: a) they failed to delay gratification, b) they were unable to evaluate the value of each reward, or c) their social learning history has taught them that it is better to take immediate rewards rather than risk missing out altogether. A critical difference between each cognitive route is the assumption that people want to inhibit the immediate reward impulse because their ultimate goal is to secure a better long-term outcome.

However, in a recent study, Kidd, Plameri and Aslin (2013) showed that the amount of time children were willing to delay gratification was modulated by uncertainty about future rewards. Kidd and colleagues manipulated the experimental context in such a way that the experimenter appeared reliable or unreliable in regards to delivering the promised reward. Children in the reliable condition waited twice as long as children in the unreliable condition for the promised reward: an unreliable environment led children to seize opportunities for reward immediately in case another opportunity was not presented. In this sense, although taking the immediate reward is a behaviour consistent 
with reward sensitivity definitions of impulsivity, in reality such behaviour was actually caused by deliberate choice based on previous learning that the best short and long-term outcome is to seize immediate reward. As outlined in Moffitt's (1993) LCP theory, it is common for offenders on the LCP path to develop in the context of unstable, unpredictable environments. Therefore, deliberate choice to seize immediate reward could explain impulsive-looking behaviour for LCP offenders better than explanations based on impairments in cognitive impulse control processes, such as reward sensitivity.

\subsection{The importance of assessing cognitive impulse control in adult offender samples}

The importance of assessing cognitive mechanisms of impulse control is aptly illustrated by the finding that LCP men were less impulsive, but more antisocial, than AL men by the age of 26 (Moffitt, Caspi, Harrington, \& Milne, 2002). Without assessing cognition based on theoretically informed frameworks, a number of competing explanations could account for this finding, with no way of establishing the validity of different explanations. One possible explanation is that by age 26 , cognitive impulse control was still impaired for LCP men, but they had developed behavioural strategies to compensate for that impairment, and therefore presented as less impulsive than AL men. However if this were true, the question remains as to why LCP offenders did not apply the same compensatory strategies to other areas of their life that remained highly dysfunctional (e.g., interpersonal relationships, employment and antisocial behaviour).

Another possibility—derived from Moffitt's assertion that cognitive impulse control impairment is manifested as impulsive personality - is that lower impulsive personality was the result of more fully developed cognitive impulse control skills (Moffitt, 1993). A large body of research shows a clear dose-response relationship 
between increasing age and the maturation of neural areas that facilitate better impulse control (see Romine \& Reynolds, 2005 for a review). Likewise, self-report and behavioural evidence from cross-sectional and longitudinal studies show that impulsivity declines linearly from childhood into the third decade of life (e.g., Steinberg, Albert, Cauffman, Banich, Graham \& Woolard, 2008; Vaidya, Latzman, Markon \& Watson, 2010). Whilst previous authors have argued that the types of neuropsychological deficits that affect impulse control and underlie LCP risk are never really corrected (e.g., Raine et al., 2000, 2005), perhaps it is more likely the development of impulse control is delayed rather than indefinitely impaired for LCP offenders. However, given the different skill domains necessary for cognitive impulse control (i.e., decision making, perceptual and motor impulse control), it is unclear whether each domain would mature at the same, or different rates. A delayed maturation hypothesis raises the possibility that the contribution of cognitive impulsivity to criminal risk might change as a function of developmental phase, making its strongest contribution in younger years but reducing in adulthood.

In the context of Attention Deficit Hyperactivity Disorder (ADHD)—a disorder partly characterised by dysfunctional impulsivity — Soper (2001) suggested that underdevelopment of the frontal lobes is prominent during childhood, but that patients catch up anatomically and tend to perform much more proficiently on neuropsychological tasks by adolescence and adulthood. If this were true, the question still remains as to why many areas of life were dysfunctional for LCP offenders at age 26 despite maturation of the neural mechanisms that support impulse control: perhaps it is best explained by the fact that sub-optimal impulse control is only one risk factor in a range of many possible 
risk factors that maintain criminal risk for LCP offenders (Moffitt, 1993). However, another possibility is that even if neuropsychological functioning was relatively normal, behavioural abnormalities did not remit. Studies examining the developmental trajectories of ADHD symptomatology show that adults displaying the dysfunctional behavioural characteristics of ADHD do not always show co-morbid deficits on neuropsychological tests (McHale, 2010). If variables unrelated to neuropsychological functioning can account for the behavioural symptoms of adults with ADHD, perhaps variables unrelated to impaired cognitive impulse control are functioning as maintenance factors for LCP risk in adulthood? As Reynolds and Horton (2008) recommend, executive functioning in clinical groups displaying dysfunctional behaviour should be periodically re-evaluated (i.e., at least every 2 years), to investigate the effect of prefrontal maturation and neuropsychological functioning in the presence of continued behavioural abnormalities.

\subsection{Where to from here?}

Developmentally, there are reasons to suspect that LCP offenders might initially be delayed in developing cognitive impulse control, but that impulse control may improve in adulthood: though only to a point where ability is still ranked below that of the general population. Without assessing cognitive mechanisms of impulse control, it is unclear whether adult criminal risk is maintained by cognitive impairment or nonimpulsive causal mechanisms: perhaps offenders deliberately choose crime because of the financial benefits, power, excitement and respect it provides (Brezina \& Topalli, 2012; Walters, 2000, 2003), or perhaps LCP offenders' criminal lifestyles diminish 
access to social opportunities that serve to foster further development of impulse control in adulthood.

Different mechanisms have important_-yet unique—implications for adult offender rehabilitation interventions. If the development of cognitive impulse control is simply delayed in LCP offenders, but the skills still have the potential to improve, rehabilitation interventions designed to retrain and strengthen mechanisms of cognitive impulse control would be promising. However if cognitive impulsive control mechanisms mature by adulthood and function normally, but LCP offenders deliberately choose to maintain their antisocial lifestyles, intervention resources would be better allocated to restructuring antisocial beliefs and developing prosocial alternatives to antisocial behaviour.

Cognitive assessment methods have been criticised for their lack of ecological validity, and for only capturing functioning at one point in time, in one specific context (e.g., Amieva, Phillips \& Della Sala, 2003; Barkley, 1991; Chan, 2001; Norris \& Tate, 2000; Ready, Stierman, \& Paulsen, 2000; Wilson, 1993). Indeed, Chaytor (2004) found that taking into account contextual factors, such as environmental cognitive demands and compensatory strategy use, accounted for unique variance in everyday executive ability over and above scores on traditional cognitive impulse control assessment methods. However, despite the proposed limitations of cognitive assessment methods, if the neuropsychological deficits that disrupt impulse control are as pervasive and stable as Moffitt's (1993) LCP theory would predict, it is expected that valid, reliable cognitive assessment methods should be able to detect those deficits at any point in time in experimentally-controlled assessment contexts. 
In a correctional setting, impulsivity is primarily assessed using personality and behavioural methods. It is suggested that the addition of cognitive assessments to complement these traditional methods would help to develop theoretical frameworks regarding the contribution of impulsivity to adult criminal risk in three important ways. First, the experimental control inherent to cognitive methods allows more reliable isolation of causal explanations of behaviour (i.e., cognitive impulse control impairment versus antisocial attitudes). Second, cognitive assessments of decision-making, perceptual and motor impulse control skills allows for unique patterns of impairment to be established. Currently, there is no theoretical or empirical guidance regarding the ecological validity of cognitive assessment methods, or which cognitive impulse control skill domains are most important to focus on in the risk assessment process. Such guidance is imperative because the operation of different causal mechanisms underlying impulsive behaviour will have different implications for clinical work (Enticott \& Ogloff, 2006; Polaschek \& Collie, 2004). Therefore, third, and most importantly, a theoretically and empirically informed understanding of cognitive impulse control impairment in adult offenders allows for better adherence to the need principle of effective offender intervention: the most relevant risk factors for each individual offender is accurately identified in the risk assessment process and targeted effectively during treatment interventions (Andrews \& Bonta, 2006).

Evenden (1999) stated that: "researchers interested in the personality trait of impulsivity, in the experimental analysis of impulsive behavior, in psychiatric studies of impulsivity or in the neurobiology of impulsivity form largely independent schools, rarely cite one another's work, and consequently rarely gain any insight into their own 
work from the progress made by others"' (pp. 348-349). With this observation in mind, I adopted Bechara's (2005) neurocognitive framework. Bechara's framework was developed to guide exploration of dysfunctional decision-making, perceptual and motor impulse control in clinical populations that display similar dysfunctional impulsive behaviour - though not necessarily of a criminal nature - to offenders. The adoption of Bechara's neurocognitive framework provides critical theoretical guidance for conceptualising and assessing cognitive mechanisms of impulse control in a correctional setting. The following chapter outlines the central tenets of Bechara's neurocognitive framework and the specific research aims of each study in this thesis that Bechara's framework helps to answer. 


\section{Chapter 3}

\section{Bechara's framework: A dual systems perspective}

\subsection{Impulsive and reflective systems}

To live adaptively, we must consider both short and long-term consequences of behaviour, and compare those consequences against our own personal standards and desires (Baumeister, Heatherton \& Tice, 1994). This type of cost-benefit analysis is generally called decision-making (Noel, Van Der Linden \& Bechara, 2006). Translating choice into actual behaviour involves selecting between many action plans - maintaining some whilst inhibiting others - which relies on mechanisms of perceptual and motor impulse control. According to Bechara (2005), engaging in the type of reflection that allows behaviour to be driven by long-term desires relies on the integrity of emotional and cognitive neural mechanisms within two separate, but interacting neural systems: an impulsive system that is dominated by subcortical mechanisms that link specific stimuli with rewards and punishments, and a reflective system that is dominated by prefrontal mechanisms that regulate behaviour.

\subsection{The impulsive system}

Responses in the impulsive system are triggered automatically by stimuli in the immediate environment (Bechara, Dolan \& Hindes, 2002). The impulsive system is the pleasure-seeking system that generates bottom-up, pleasure or pain signals associated with immediate prospects of an option; that is, the impulsive system signals which options will feel good (pleasure), or bad right now (pain; Damasio, 1996). Bechara and 
colleagues refer to pain and pleasure signals as somatic markers: bodily sensations akin to a hunch or gut feeling that a particular response option is good or bad based on previous experience with that behaviour and its consequences (Bechara, Damasio, Damasio, \& Anderson, 1994).

Although contested by some researchers (e.g., Maia \& McClelland, 2004), it is suggested that somatic markers generate physiological responses that serve to bias decision-making processes even if people have no awareness of the basis for their decision (Bechara \& Damasio, 2002; Bechara, Damasio \& Damasio, 2000; Lowenstein \& Lerner, 2003). Specifically, laboratory-based studies show that after initial periods of exposure to reward and punishment contingencies, neurologically healthy participants generate skin conductance responses as a reaction to reward and punishment. In addition, once experienced with reward and punishment contingencies, neurologically healthy participants begin to generate skin conductance responses when anticipating response options, which gradually become more pronounced before choices with a high likelihood of punishment (Bechara, Damasio \& Damasio, 2000). The normal development of these physiological responses facilitates approach toward advantageous, rewarding choices and avoidance of risky, high-punishment choices (Bechara \& Damasio, 2002; Bechara, Damasio \& Damasio, 2000).

Processing in the impulsive system is continuous; the system is always switched on and information is processed quickly, effortlessly and automatically. Processing in the impulsive system is effortless because it relies on the associative networks developed from learning experiences between a stimulus and its affective properties (Strack \& Deutsch, 2004). In the impulsive system the amygdala is the key neural region for 
generating somatic markers based on affective properties of a stimulus (Bechara, 2005).

In patients with damage to the amygdala, the process of linking stimuli with their affective properties is disrupted. Accordingly, previous studies show that amygdala damaged patients fail to generate normal physiological responses during the decisionmaking process; thus, choices are bound by options that are immediately rewarding but ultimately punishing in the long-term (Bechara, Damasio, Damasio \& Lee, 1999).

\subsection{The reflective system}

The reflective system also generates somatic markers. Sometimes the somatic markers generated by the impulsive and reflective system are compatible; behavioural options that will satisfy immediate desires will also satisfy longer-term desires. However sometimes, the somatic markers generated by each system are incompatible. When the signals are incompatible, successful functioning of the reflective system is necessary in order to weight the relative costs and benefits of different response options against desired short and long-term outcomes. Therefore, when the reflective system works effectively, we are able to choose according to short or long-term outcomes; if the reflective system fails, we are guided exclusively by immediate prospects of an option signaled by the impulsive system (Bechara, 2005).

The somatic markers generated by the reflective system are based on recall of previous personal experiences of reward and punishment, or from imagining hypothetical reward and punishment contingencies (e.g., how did it feel last time when I did X? I think I will feel bad if I do X again). The ventromedial prefrontal cortex (VMPC) is the key neural region that allows us to predict how something will "feel" in the future, linking emotion to the actions that initially caused the emotion (Bechara, Tranel, \& Damasio, 
2000; Bechara, Tranel, Damasio \& Damasio, 1996; Tranel, Bechara \& Denburg, 2002). Specifically, the VMPC integrates the functions of two systems. The first, involving the insular cortex and somatosensory cortices, is responsible for processing the emotional information related to somatic states triggered in memory (e.g., how did it feel last time I did X?). The second system involves working memory and executive processes of inhibition, planning and cognitive flexibility. The dorsolateral prefrontal cortex and hippocampus are key neural structures for imagining the future consequences of an action and keeping current goals in mind during the decision-making process (Bechara, 2005). Recalling emotional information and engaging the cognitive skills necessary to produce a well considered response is a slow, deliberate and effortful process, requiring a great deal of cognitive energy (Bechara, 2005). Unlike the impulsive system, which functions effortlessly, functioning in the reflective system can fail if sufficient cognitive resources are not available.

The reflective system can be hijacked by the impulsive system via two different mechanisms: 1) hyperactivity in the impulsive system which exaggerates the rewarding impact of available incentives and generates exceedingly strong somatic markers, or 2) hypoactivity in the reflective system in which the generation of, and ability to act on, somatic signals associated with future prospects of an option does not function effectively (Bechara, Noel \& Crone, 2006). For example, in the context of substance abuse, previous learning and reinforcement ensures that drugs come to acquire strong affective properties such that automatic and exaggerated autonomic responses occur in the presence of drugrelated cues. The presence of drug-related cues in the immediate environment triggers such strong responses in the impulsive system that it becomes powerful enough to 
override the functions of the reflective system. Arguably, the same processes could apply to the rewarding value of antisocial behaviour developed from reinforcement experiences (Walters, 2000, 2003).

However, functions of the reflective system can also fail because the reflective system is chronically weak, irrespective of the strength of signals generated by the impulsive system. Hypoactivity in the reflective system can exist at a trait or state level. Consistent with the central tenets of Moffitt's (1993) LCP theory, Bechara (2005) suggested that stable, trait impairments arise from genetic inheritance and/or environmental factors that cause developmental abnormalities in the cortical mechanisms that support decision-making and impulse control. As discussed in Chapter 2, compounding the issue of genetic vulnerability toward poor reflective ability is the interaction between those vulnerabilities and environmental factors such as a child's socialisation experiences. Whilst genetic variation can account for initial weaknesses in reflective system strength, continued positive socialisation, supervision and discipline from parents contribute most to enduring reflective ability (Eisenberg et al., 2005). Reflective ability is considered analogous to the workings of a muscle insofar as the more practice we get, the stronger our control skills become (e.g., Muraven, Baumeister \& Tice, 1999). Therefore, if children never learn how and why they should carefully consider behaviour before acting, arguably they will fail to practice and strengthen their reflective skills; frequently defaulting to behaviour based on immediate needs instead.

Effortful processing in the reflective system requires substantial cognitive energy; thus, temporary depletions of cognitive energy can also cause temporary weakness in reflective system strength (Muraven \& Baumeister, 2000). Examples of factors that 
deplete the cognitive resources necessary for reflection include: being under the influence of drugs and alcohol (e.g., Bechara, 2007; Fillmore, Dixon \& Schweizer, 2000; Giancola, 2000), negative mood states (e.g., Knapp \& Clark, 1991; Tice, Bratslavsky \& Baumeister, 2001), and states of high physiological arousal (e.g., Eysenck, 1982). Temporary failure of the reflective system is aligned with state-based, rather than trait explanations of cognitive impulse control impairment. Because Moffitt's LCP theory specifically emphasises the contribution of chronic, trait-based impairment in disinhibition — which is arguably caused by a chronically weak reflective system-the aim of this thesis is to focus on reflective system weakness at a trait level.

3.3.1 Mechanisms of weakness in the reflective system: Decision-making, perceptual and motor impulse control

As introduced in Chapter 1, Bechara suggested that weaknesses in the reflective system leading to impulsive behaviour can occur from impairments in one, two or all three reflective domains that facilitate impulse control: 1) the decision-making process itself whereby people fail to generate the somatic markers necessary for biasing decisions, 2) perceptual impulse control, and 3) motor impulse control. Put another way, optimal reflective functioning relies on each of these mechanisms working together. Hereafter, I refer to decision-making, perceptual and motor impulse control as independent domains, but also collectively as general mechanisms of cognitive impulse control.

According to Bechara (2005), in a normally functioning brain, it is common for all cognitive impulse control mechanisms to be recruited in the process of pondering a decision. However, under controlled experimental assessment conditions, it is possible to 
fractionate weaknesses, both within and across domains. Establishing unique patterns of impairment is a crucial step in developing theoretical frameworks for understanding the contribution of cognitive mechanisms of impulse control to criminal risk.

\subsection{Summary and research objectives}

Findings are mixed regarding the level of impairment adult offenders display on decision-making, perceptual and motor impulse control tasks and how those abilities are related to antisocial outcomes. To develop a theoretically informed understanding of the contribution of cognitive impulse control to criminal risk in adulthood, I aim to address some of the gaps identified in existing literature so far, by adopting Bechara's neurocognitive framework. A promising way to address existing gaps is via Enticott and Ogloff's $(2006$, p.9) recommendation for a three-tiered approach to the conceptualisation and assessment of impulsivity. They suggest that investigations should occur at three levels: 1) the individual level (who is impulsive?), 2) the expression level (what does the expression of impulsive behaviour look like and how do we observe and quantify that behaviour?), and 3) the causal level (the processes/mechanisms causing the behavioural expression of impulsivity).

Currently, at the first level of investigation, it is assumed that the majority of LCP offenders will be cognitively impulsive as this is one factor hypothesised to maintain criminal risk (Moffitt, 1993). However, support for this assumption is derived from personality and behavioural assessments, which capture a generalised tendency to prefer an impulsive lifestyle, rather than capturing cognitive functioning. Therefore, we can currently only sufficiently answer the question of who is impulsive at the level of personality and behaviour, but not at the causal level of cognition. The application of 
Bechara's framework and its recommended cognitive assessment tasks will help to answer the question of who is impulsive at the level of cognition.

At the second level of investigation, we currently understand the expression of impulsive behaviour to be any domain-general behaviour that appears to be ill planned and spontaneous with no regard for the consequences. However, the ability to observe and quantify that behaviour in a controlled and reliable way is currently lacking in a correctional setting because of reliance on personality and behavioural measures. Unless we directly test for a particular explanation of impulsive behaviour via an experimentally controlled response, how can we rule out non-impulsive explanations for the same behaviour? Accordingly, I will employ a cognitive assessment battery that captures functioning within Bechara's three domains of cognitive impulse control via observable, quantifiable responses. Because reliable and valid cognitive measures can isolate specific cognitive mechanisms underlying an impulsive behavioural response, the third causal level of investigation — establishing why people behave impulsively—can be achieved.

\subsection{Thesis structure}

All of the data in this thesis are from a single pre-to-post-treatment study of offenders recruited from 12 treatment cohorts from four Special Treatment Unit Rehabilitation Programmes (STURPs) across New Zealand. Entry criteria to the STURPS requires that offenders be at high risk of reoffending as determined by the New Zealand Department of Corrections actuarial risk assessment tool (see Chapter 4, General Method). A previous study showed that approximately $66 \%$ of New Zealand's high-risk offenders fit Moffitt's LCP trajectory: offenders had a well-established pattern of antisocial behaviour before 13 years of age, including first police contact by 11 years of 
age (Wilson, 2004). Accordingly, it is assumed that the offenders recruited for this thesis predominantly represent offenders on the LCP path. The following chapter (Chapter 4, General Method) describes the setting, data collection methodology and procedure used for each independent study in this thesis. Each study is preceded by its specific objective, a review of relevant research, specific method details, outline of data analysis techniques, results and discussion section.

\subsection{Study outlines and specific research questions}

Study 1 answers the questions of who is impulsive and how? First, I will empirically test Bechara's assertion that there are three, potentially dissociable domains of cognitive impulse control. Next, I will examine where adult high-risk offenders (postulated to fit Moffitt's LCP typology), perform on cognitive impulse control tasks relative to normative populations. The foci for Study 1 are based on the rationale that whilst it is important to know where certain offender groups perform relative to other offender groups, directing intervention to the most need-worthy offenders requires that we establish how offenders perform compared to normal functioning people in the general population. Without comparing offender performance to that of non-offender controls, we cannot fully understand the magnitude and clinical relevance of impairment. More specifically, Study 1 addresses the following questions: 1) What proportion of the sample performs at a clinically impaired level on tests of cognitive impulse control? 2) Are there unique patterns of impairment? 3) What proportion of the sample is impaired in more than one domain?

Study 2 investigates whether cognitive impulse control changes, following an intensive cognitive-behavioural rehabilitation programme designed to reduce multiple 
areas of criminal risk, including impulsivity. As previously discussed, LCP offenders are theorised to begin life on the 'back foot' as a function of impaired neuropsychological functioning and disadvantaged developmental environments that disrupt the development of regulatory processes like impulse control. Moffitt's LCP theory predicts that such deficits are relatively consistent across the lifespan (Piquero \& Moffitt, 2011). However, a large body of research shows improvements in impulse control with age. Furthermore, previous studies with offenders have not always assessed cognitive mechanisms of impulse control; therefore, more work is needed to establish the validity of predictions about impairments in cognitive mechanisms of impulse control based on Moffitt's LCP theory. Are expected cognitive impulse control impairments empirically supported? Is there evidence for plasticity in these functions? What happens to impulse control following an intensive cognitive-behavioural rehabilitation programme aimed at increasing behavioural regulation?

Moffitt's LCP theory is founded on the prediction that variation in impulse control should account for variation in criminal risk (Piquero \& Moffitt, 2011). However, findings from investigations that test this prediction are inconsistent. Accordingly, Study 3 explores how cognitive impulse control is associated with dynamic criminal risk; and specifically, the ability to alter dynamic risk. Moffitt's LCP theory also predicts that inhibitory deficits and negative emotionality contribute to difficult temperament (Moffitt, 1993). Thus, Study 3 also examines associations between cognitive impulse control, anger, and anger control; again, specifically investigating the relationship between cognitive impulse control and treatment change in anger, and anger control. Collectively, 
Study 3 aims to contribute to the sparse theoretical literature regarding impulsivity and its relationship with dynamic risk overall, and with other risk factors. 


\section{Chapter 4}

\section{General Method}

\subsection{Setting}

Participants were recruited during a two year period between 2009 and 2011 from four different prison-based Special Treatment Unit Rehabilitation Programmes (STURPs) across New Zealand: The Violence Prevention Unit ${ }^{2}$ at Rimutaka Prison, Wellington; Matapuna at Christchurch Prison, Christchurch; Karaka at Waikeria Prison, and Puna Tatari at Springhill Prison both in the Waikato region. During this period, the Violence Prevention Unit exclusively recruited offenders with an extensive history of violent offending, whilst the other STURPs recruited a mixture of general, violent or adult sexual offenders (see Polaschek \& Kilgour, 2013 for a recent review of New Zealand STURPs) ${ }^{3}$.

The STURPs are 30 to 40-bed, low-medium security units within the prison. Attendance at the therapy programme is voluntary; however the New Zealand Parole Board commonly recommends attendance to increase potential eligibility for parole. The admission criteria across all STURPs includes offenders with high static risk scores (at least a $70 \%$ chance of returning to prison in the five years following release) ${ }^{4}$, a lowmedium or minimum internal security status, and enough time left on the prison sentence to complete the programme (Polaschek \& Kilgour, 2013).

The STURP treatment model is consistent with the Risk-Need-Responsivity

\footnotetext{
${ }^{2}$ Since renamed Te Whare Manaakitanga.

${ }^{3}$ The STURP description in this thesis is based on the period during which research data was collected. There have been, and continue to be a number of changes to the STURPs.

${ }^{4}$ Based on the RoC*RoI (Bakker, O’Malley \& Riley, 1998). The RoC*RoI is an actuarial risk assessment tool used by the New Zealand Department of Corrections. Based on criminal history variables, the $\mathrm{RoC}^{*} \mathrm{RoI}$ estimates serious reconviction risk leading to reimprisonment over the 5 years following release. A RoC* $\mathrm{RoI}$ of $\geq .7$ is considered high-risk.
} 
model of offender rehabilitation (Andrews \& Bonta, 2006). Opportunities for pro-social change occur via attendance of the core cognitive-behavioural treatment programme embedded within a social learning-based therapeutic community (Polaschek \& Kilgour, 2013). Within treatment sessions, and more generally in the treatment unit, treatment participants are encouraged to develop new skills by actively practicing and applying skills to their interactions with other offenders, custodial and therapy staff.

The core treatment programme is intensive: 99 sessions of 2.5 hours, delivered over 25 weeks (Polaschek \& Kilgour, 2013). There are three overarching treatment phases: orientation, knowledge and skill building, and preparing for post-treatment transfer or release (Polaschek \& Kilgour, 2013). Specifically, within the three treatment phases, the core treatment programme covers 8 manualised modules: 1) Getting to know one another and identifying background and motivational factors to offending; 2) Distress Tolerance: Identifying thoughts and beliefs that foster violent behaviour; 3) Offence Mapping: Identifying offence patterns for use in treatment and for safety planning; 4) Looking at thinking: Methods for changing how to deal with problems including mindfulness; 5) Emotions and self-control: Mood and anger management; 6) Substance Abuse; 7) Getting along with others: Communications and relationship skills; 8) Safety planning for release: how to cope, and establishing a support network. Either side of the core treatment programme, treatment participants undergo pre and post-treatment assessment. Offenders in this research were recruited during these pre-post treatment assessment periods. 


\subsection{Participants}

\subsubsection{Offender sample}

Ninety-one male prisoners were approached for voluntary participation in the research. Of those, $14(15.4 \%)$ declined participation. Of the 77 consenting inmates, 62 were currently imprisoned for a violent/non-violent index offence, and 15 for a sexual offence against adult victims. Nearly half $(n=33)$ of the consenting inmates were recruited from the Violence Prevention Unit, 19 were recruited from Puna Tatari, 6 were recruited from Karaka, and the remaining 19 from Matapuna. The mean probability of reoffending and re-imprisonment within 5 years after release was $69 \%(S D=18 \%)$, confirming the high static risk of the sample. Participants were predominantly New Zealand Maori (52.3\%) or Pakeha (30.8\%) with the remainder of the sample of Pacific Island or Asian ethnicity. On average participants were 32 years old $(S D=9.20$; range 19-59 years) with a mean full-scale IQ of $94.7(S D=13.92)$. Of the 77 consenting inmates, $58(75 \%)$ completed the treatment programme, and $19(25 \%)$ did not. There were no significant differences in criminal risk, age or IQ between programme completers and non-completers. Similarly, there were no significant differences in criminal risk or age between offenders who declined or consented to participate in the research. Comparison on IQ could not be conducted due to incomplete IQ data for some offenders who declined participation.

\subsubsection{University student sample}

Normative data was not available for one task in the assessment battery (Stop Signal). As per the recommendations of the task developers, a sample of male university students $(N=26)$ was recruited to complete the task to obtain normative data (F. 
Verbruggen, personal communication, May 19, 2009). In a single session, students completed the Stop Signal task and two short impulsivity questionnaires ${ }^{5}$. All students were completing a first year psychology course, which required mandatory participation in psychological research. Students received a one hour credit towards their mandatory participation tally for completing the Stop Signal task and the questionnaires (see Appendices 5, 6 and 7 for a copy of the information, consent and debriefing forms). Preliminary group comparisons revealed that offenders were significantly older than students (offenders Mage $=32$ years, $S D=9$; students Mage $=25$ years, $S D=8: t(89)=$ $3.60, p<.01)$.

\subsection{Materials}

The selection of tasks was based on Bechara's recommendations of neuropsychological measures that most closely align with decision-making, perceptual and motor impulse control (e.g., Bechara, Noel \& Crone, 2006). If a recommended measure was unavailable, a reliable, well-validated alternative was chosen (Lezak, Howieson, Bigler \& Tranel, 2012). A total of five tasks were included in the assessment battery: one task to assess decision-making (Iowa Gambling Task), two tasks to assess perceptual impulse control (Colour-word Interference Test; Comprehensive Trail-making Test), and two tasks to assess motor impulse control (Continuous Performance Test; Stop Signal Task). Three tasks were computerised (Iowa Gambling Task, Continuous Performance Test, Stop Signal Task), and two were not (Colour-Word Interference Test, Comprehensive Trail-making Test). The Colour-Word Interference Test required verbal responses, and the Comprehensive Trail-Making Test required participants to draw lines

\footnotetext{
5 The questionnaires included the Barratt Impulsivity Scale (BIS-11; Patton, Stanford, \& Barratt, 1995), and the Behavioural Inhibition System/Behavioural Activation System scales (Carver \& White, 1994). Questionnaire data is not reported in this thesis.
} 
connecting stimuli on a page. All computerised tasks were presented on a Dell PC Laptop (Latitude D800, PP02X; 15” screen size).

\subsubsection{Decision-making domain: Iowa Gambling Task, computerised version ${ }^{6}$ (IGT;} Bechara, 2007).

Bechara and colleagues developed the IGT to assess cognitive and affective mechanisms of decision-making. For advantageous decision-making, people require intact cognitive reasoning skills to evaluate the risks and benefits associated with different options, the ability to retrieve that knowledge from memory, and the ability to think about multiple response options and their consequences at once. Affective processes interact with these cognitive processes via the generation of somatic markers (see Chapter 3) that signal which response options "feel" good or bad based on experiences of reward and punishment associated with specific responses. The IGT was designed to capture these cognitive and affective processes.

Specifically, the IGT simulates the types of choice contexts encountered in every day decision-making that involve making choices under conditions of risk and ambiguity (Stocco, Fum \& Napoli, 2009). The two underlying assumptions in the IGT are that advantageous decision-making relies on choosing options that are most beneficial in the long-term —a process influenced by the normal generation of somatic markers-and that monetary reinforcement is equally desired and rewarding for everyone (Bechara, 2007).

\subsubsection{IGT task description}

On the computer screen participants are presented with four decks of cards (A, B, C, D; 60 cards per deck) and an opening balance of $\$ 2000$. Participants are told that the

\footnotetext{
${ }^{6}$ The IGT was originally administered using decks of paper cards. Rewards have also been presented as real reward or 'play money'. There are no differences in task reliability as a function of presentation modality or type of reward (Buelow \& Suhr, 2009).
} 
aim of the game is to maximise profits and minimise losses, but they are not told explicitly how to win or how many card selections there will be before the game ends. Participants are told that their opening balance is a loan which must be paid back to win, or at least break even during the game.

Participants take as much time as they need to select a card from any deck using the computer mouse. Every card selection results in a win with the winning amount shown on-screen after the card selection. But unpredictably on some trials, a win is accompanied by a loss, which is also shown on-screen (e.g., "YOU WIN \$50, but LOSE $\$ 100)$.

As shown in Table 1, the card decks differ in reward and punishment outcome and frequency. Regarding outcome, Decks A and B are disadvantageous because they yield high rewards but even larger losses, therefore continued selections from Decks A and B result in an overall net loss. Alternatively, Decks C and D are advantageous because they yield lower rewards than Decks A and B but even lower losses, resulting in an overall net gain (Bechara, Damasio, Damasio \& Anderson, 1994). Regarding frequency, Decks B and D have the lowest loss frequencies, whilst Decks A and C have the highest loss frequencies.

As per the IGT Manual (Bechara, 2007), IGT performance is evaluated according to outcome criteria only. The standard administration parameter of 100 card selections was used in the present study. The average completion time for the task is 20 minutes $^{7}$. All 77 pre-treatment participants completed the gambling task.

\footnotetext{
${ }^{7}$ The gambling task can take more or less time depending on each participant's deliberation time between card selections.
} 
Table 1. Pay-off scheme for each IGT card deck

\begin{tabular}{lcccc}
\hline & Deck A & Deck B & Deck C & Deck D \\
& $\begin{array}{c}\text { Bad/frequent } \\
\text { loss }\end{array}$ & $\begin{array}{c}\text { Bad/infrequent } \\
\text { loss }\end{array}$ & $\begin{array}{c}\text { Good/frequent } \\
\text { loss }\end{array}$ & $\begin{array}{c}\text { Good/infrequent } \\
\text { loss } \\
\text { Reward/trial }\end{array}$ \\
100 & 100 & 50 & 50 \\
$\begin{array}{l}\text { Number of } \\
\text { losses/10 cards }\end{array}$ & 5 & 1 & 5 & 1 \\
$\begin{array}{l}\text { Loss/10 cards } \\
\text { Net outcome/10 }\end{array}$ & $-\$ 1250$ & $-\$ 1250$ & $-\$ 250$ & $-\$ 250$ \\
cards & $-\$ 250$ & $-\$ 250$ & $\$ 250$ & $\$ 250$ \\
\hline
\end{tabular}

\subsubsection{IGT outcome scores}

The primary performance outcomes in the gambling task include: Total net scores ( $C D-A B, 100$ card selections), block net scores $(C D-A B$, per block) and total number of cards selected from each deck (provided per block of 20 selections, or across all 100 selections). Additional outcomes generated by the IGT score report include the total amount of money won (money won - money lost and borrowed) and response time between card selections. Response time between card selections reflects participants' approach to decision-making; fast response times and disadvantageous choices support an impulsive decision-making style (IGT Manual; Bechara, 2007, p. 10).

Although some studies have used total money won and response time to interpret gambling task performance, there are no normative data available for these outcomes. Additionally, they are not yet shown to have more predictive validity than the primary outcomes (IGT Manual; Bechara, 2007, p.9). Therefore total net scores, block net scores 
and total number of cards selected from each deck were selected as the primary

performance indicators in this study.

To assist performance interpretation, total and block net scores and number of card selections from each deck are compared to normative data $(N=932)$. Raw scores are converted to $T$-scores $(M=50, S D=10)$ with corresponding percentiles. The following categories are recommended for interpreting performance: $T \geq 45=$ Non-impaired range, $T$ 40-44 = Below average range, $T \leq 39=$ Impaired range (IGT Manual; Bechara, 2007, p.8).

As stated in the IGT manual (p.7), there are two goals to neuropsychological test interpretation: 1) to make diagnostic inferences (represented by demographicallycorrected scores) and 2) to make inferences about the tested ability more globally regarding every day functioning (represented by U.S. Census-matched scores). My objective is more closely aligned with making diagnostic inferences; therefore, I interpreted offender scores against the demographically-corrected scores (corrected for gender, age, and education).

\subsection{Total net scores}

Total net scores are calculated from 100 card selections by subtracting total disadvantageous selections from total advantageous selections $(\mathrm{CD}-\mathrm{AB})$. As a general guideline, positive total net scores reflect advantageous decision-making, indicating that most choices were made from decks with the best outcome: highest net gain and lowest net loss overall. Negative total net scores reflect disadvantageous decision-making, indicating that most choices were made from decks with the worst outcome: lowest net gain and highest net loss overall. The magnitude of negative net scores determines 
whether performance is below average or significantly impaired (i.e., a total raw net score of at least -19 or below, which equates to $T \leq 39$ ).

Using total net scores as the only outcome to interpret decision-making on the IGT has been criticised because it collapses choices across all five blocks of card selections. This is problematic because choices in earlier blocks reflect decisions made under conditions of ambiguity, while choices made in later blocks reflect decisions made under conditions of risk (Beulow \& Suhr, 2009). As supported by neuroimaging studies, different neural mechanisms support decisions under conditions of ambiguity versus conditions of risk (Beulow \& Suhr, 2009; Krain, Wilson, Arbuckle, Castellanos \& Milham, 2006). Analysis of block net scores provides a more fine-grained method for evaluating the choice patterns underlying a participant's total net score (e.g., a positive or negative learning curve or random performance).

\subsection{Block net scores}

The total of 100 card selections can be split by performance across 5 blocks of 20 card selections (e.g., Net $1=$ card selections $1-20$, Net $2=$ card selection $21-40$ and so on). Like total net scores, block net scores are calculated by subtracting disadvantageous selections from total advantageous selections, per block $(\mathrm{CD}-\mathrm{AB})$. Positive block net scores indicate advantageous decision-making whilst negative block net scores indicate disadvantageous decision-making. Within the first blocks, negative block net scores are normal as participants are still gauging which decks are rewarding and punishing (Bechara, 2007; Wagar \& Dixon, 2006).

Advantageous performance is reflected by block net scores that steadily increase as the task progresses, indicating a positive learning curve. Disadvantageous performance 
is reflected by block net scores that do not improve as the task progresses, block net scores that decrease as the task progresses, or block net scores that fluctuate erratically indicating random choices (IGT Manual; Bechara, 2007)

\subsection{Card selections from each deck}

Decks A and B return large reward but even larger losses and the size of the losses increase as the task progresses. Decks C and D return smaller rewards but even smaller losses and the size of the wins increase as the task progresses. Therefore, in the long run, relatively more choices from Decks C and D result in a net win whilst relatively more choices from Decks A and D result in a net loss. Advantageous performance is therefore indicated by relatively more selections from Decks C and D than A and B. Disadvantageous performance is indicated by relatively more selections from Decks A and $\mathrm{B}$ than $\mathrm{C}$ and $\mathrm{D}^{8}$.

\subsubsection{IGT reliability}

One of the major criticisms of the IGT is lack of reliability data (Buelow \& Suhr, 2009). The professional manual is silent on the issue of test-retest reliability (Bechara, 2007). Potential practice effects have been illustrated by some studies showing performance improvements with re-test delays ranging from 1 to 25 days (e.g., Lejuez et al., 2003; Verdejo-Garcia \& Pérez-Garcia 2007). Regarding construct validity, the IGT is intended to assess the "level of decision-making capacity of an individual in relation to the general population" and to "obtain information that supports a diagnosis" (Bechara, 2007, p.7). Evidence from research using the IGT with a range of clinical populations and

\footnotetext{
${ }^{8}$ Emerging evidence shows that neurologically healthy participants prefer low loss frequency decks (B, D) as well as decks with the best long-term outcomes (C, D). Thus it is argued by some researchers that choosing according to options with low frequency punishment also indicates advantageous decision-making (e.g., Hortsmann, Villringer \& Neumann, 2012).
} 
neuroimaging studies generally supports the construct validity of the IGT and its ability to detect abnormalities in the cognitive and affective processes that contribute to advantageous decision-making (see Buelow \& Suhr, 2009 for a comprehensive review). However, researchers have suggested that further work is needed to establish convergent validity, ecological validity and the influence of other variables on IGT performance, such as personality characteristics and state mood (Buelow \& Suhr, 2009).

\subsubsection{Perceptual impulse control domain: Delis-Kaplan Executive Function System:}

Colour-Word Interference Test: (D-KEFS CWIT; Delis, Kaplan \& Kramer, 2001).

Bechara's (2005) framework operationalises perceptual impulse control as the ability to resist and inhibit cognitive interference from habitual but contextually inappropriate thoughts, and flexible set shifting. To represent this domain, the DelisKaplan Colour-word Interference Test and the Comprehensive Trail Making Test were selected because both tests include inhibition and set shifting conditions.

The D-KEFS is a battery of nine neuropsychological tasks. The CWIT is one task from the battery based on the standard Stroop test. The CWIT has four conditions: 1) Colour Naming 2) Word reading 3) Inhibition and 4) Inhibition/switching. The colour naming and word reading conditions assess the key component skills necessary for the higher-level tasks of inhibition and inhibition/switching. All conditions are timed; participants are instructed to complete each condition as quickly and accurately as possible.

Condition 1 (colour naming) presents 50 coloured squares (red, blue, green) arranged linearly across the page. Participants are required to read across the page from left to right naming the colour of each square. Condition 2 (word reading) presents 50 
colour words printed in black ink. Participants are required to read each word aloud.

Condition 3 (inhibition) presents participants with colour words printed on the page in an ink colour that is incongruent with the word (i.e., the word 'green' printed in red ink).

Participants must inhibit the habitual response to read the word and name the incongruent ink colour instead. Condition 4 (inhibition/switching) presents participants with colour words: some with a box around the word, and some without a box around the word. Participants must flexibly switch between two different responses according to the presence or absence of a box around the word. When the word has a box around it, participants simply read the word within the box (word reading). When there is no box, participants must inhibit the habitual response to read the word and name the ink colour instead (inhibition). The average completion time for the CWIT is 10 minutes. Two pretreatment participants refused to complete the task; 75 pre-treatment participants completed the task.

\subsubsection{CWIT outcome scores}

The primary outcome scores are raw completion times (measured in seconds) for each condition. Raw completion times are converted to age-corrected scaled scores $(M=$ $10, S D=3$ ). Scaled scores for Conditions 1 and 2 are summed to create a composite scaled score combining colour naming and word reading abilities.

The calculation of contrast scores allows for isolation of disproportionately poorer performance on higher-level conditions (inhibition and inhibition/switching) relative to baseline skills (colour naming and word reading). Contrast scores between 8-12 represent equivalent performance in baseline and higher-level conditions. A contrast score of 13 or above represents disproportionately better performance in higher-level conditions relative 
to baseline, whilst a contrast score of 7 or less represents disproportionately worse performance in higher-level conditions relative to baseline conditions. However, the reliability of using contrast scores to evaluate CWIT performance has been challenged (Crawford, Sutherland \& Garthwaite, 2008). Error analysis also provides additional optional outcomes to assist performance interpretation. Both corrected and uncorrected errors are tallied during testing. The raw number of corrected and uncorrected errors is compared against normative data (percentiles and scaled scores).

\subsubsection{CWIT reliability}

All D-KEFS tests were standardised on a nationally representative, stratified sample of 1750 non-clinical children, adolescents, and adults, ages 8-89 years old (Delis, Kaplan \& Kramer, 2001; Homack, Lee \& Riccio, 2005). For the average age of participants in this thesis, the composite score internal consistency value is $0.82(30-39$ years). The test-retest correlations for each condition are in the moderate to high range (0.49-0.86; average time between administrations of $25+/-12.8$ days). Re-test scores are slightly higher suggesting improved performance following exposure to the test.

\subsubsection{Comprehensive Trail Making Test (CTMT; Reynolds, 2002)}

The CTMT includes five timed trails assessing visual cue perception, mental flexibility, alternating attention, and behavioural inhibition. Each trail becomes gradually more difficult and introduces increasing levels of distraction. All trails involve drawing lines to connect Arabic numerals or written stimuli. Trails 1-3 constitute simple trails with relatively fewer distractions than the complex trails (Trails 4-5). Specifically, Trails 1-3 all involve joining the numerals $1-25$ as quickly as possible without skipping any numbers in the sequence. Trail 1 includes no distractors, in Trail 2 empty distractor 
circles are added to the page and in Trail 3 empty distractor circles and extra picture circles are added to increase the level of distraction. Trail 4 is slightly different to initial trails whereby the numbers 1-25 must be joined but some numbers are presented in Arabic numerals and the rest are written in their full word form (e.g., twelve) requiring flexible shifting between perceptual sets. Trail 5 involves higher-level set shifting by joining the numbers 1-25 with their corresponding letter in the alphabet from A-L (i.e., 1A-2-B-3-C). Empty distractor circles are also added to the page for Trail 5. Therefore, Trails 4 and 5 both assess flexible set shifting but the added complexities of Trail 5tracking and shifting between numerals and letters - make Trail 5 more difficult than Trail 4 (Reynolds, 2002).

Trails are presented in numerical order, because simple trails must be passed before participants attempt complex trials. A brief practice example is completed by participants before Trails 1-3 (to practice simple trails) and before Trails 4-5 (to practice complex trails) to ensure participants understand task instructions before beginning the test trails. The average completion time (including practice examples) for the task is between 5-12 minutes. All 77 pre-treatment participants completed the Trail-making test.

\subsubsection{Trail making outcome scores}

Raw completion times for each trail (measured in seconds) are compared against normative data $(N=1964)$. Raw completion times are converted to $T$-scores $(M=50, S D$ $=10$ ) with corresponding percentiles. The $T$-scores from all 5 trails are pooled to provide a Composite Index score to gauge overall performance. $T$-scores are interpreted according to the following categories: $T$-scores $\geq 43$ represent average to very superior ( $T$ $>70)$ performance. $T$-scores between $36-42$ represent below average performance, $T$ - 
scores between 30-35 represent mildly to moderately impaired performance, and $T$-scores $<30$ represent severely impaired performance.

\subsubsection{Trail making reliability}

The CTMT shows good internal consistency for the Composite Index score (.91) and individual trail scores $(.68-.74)$. Good test-retest reliability for the Composite Index score $(.84)$ and individual trail scores $(70-.78)$ was observed for 30 adults tested twice with one week between testing. In an investigation of the validity of the CTMT, Smith and colleagues (2008) showed the CTMT has good internal consistency, and divergent validity, but relatively less support was found for convergent validity. They also found that the CTMT had good discriminant validity: the CTMT Composite Index score was able to differentiate clinical and non-clinical groups with a large effect size (Smith et al., 2008).

4.3.4 Motor impulse control domain: Conners' Continuous Performance Test-II (CPT-II; Conners, 2002).

Bechara's (2005) framework operationalises motor impulse control as the inhibition of a pre-potent motor response. To represent this domain, the Conners' Continuous Performance Test (CPT) and Stop Signal task were selected to assess two different components of inhibition. The CPT requires rapid evaluation of a stimulus for the purpose of deciding whether to respond or not. On the other hand, the Stop Signal task assesses the speed of stopping an already-initiated motor response (Logan, Schachar \& Tannock, 1997).

In the CPT, participants attend to a series of rapidly presented, white capital letters on a black computer screen (approximately 1 inch in size, bold face). The letters 
are presented individually, for $250 \mathrm{~ms}$ at three different inter-stimulus intervals $(1,2,4$ seconds). Participants must respond to all letters of the alphabet other than the letter X by pressing the spacebar button on the computer keyboard. Responses must be inhibited to all presentations of the letter $\mathrm{X}$. The task has six equal blocks; each block includes 20 trials at each of the three inter-stimulus intervals, for a total of 360 trials. Before beginning the test blocks, a practice administration with identical requirements as the test blocks was provided for all participants. As per the standard CPT protocol, the practice administration ran for 70 seconds; however the practice test could be stopped prematurely as soon as it was clear a participant understood the task (CPT Manual; Conners, 2002, p.15). No participants required a second practice administration in this study.

The average completion time for the CPT is 14 minutes. Two pre-treatment participants did not complete the CPT due to unavoidable prison interruptions, and 1 participant could not complete the task due to computer failure: a total of 74 pretreatment participants completed the CPT.

\subsubsection{CPT outcome scores}

The outcome scores pertaining to impulsive responding are commission and omission errors and hit reaction time (CPT Manual; Conners, 2002, p.29). Commission errors occur when a participant makes a response to the non-target letter X. Omission errors occur when a participant fails to respond to target letters. Hit reaction time is the time taken for participants to respond to target letters (CPT Manual; Conners, 2002, p.29). Specifically, impulsive performance is operationalised as a high number of commission errors and relatively fewer omission errors, and fast hit reaction times. 
The raw number of commission and omission errors and raw hit reaction time are converted to age-corrected $T$-scores $(M=50, S D=10)$ with corresponding percentiles. All outcome scores can be compared against non-clinical $(N=1920)$, ADHD $(N=378)$ and neurologically impaired ( $N=223)$ samples. Following common convention, I will compare scores against the non-clinical normative sample (CPT Manual; Conners, 2002).

For commission and omission errors, all $T$-scores below 54 represent average to very good performance. Any $T$-scores above 55 represent the cut-off for atypical performance $(T 55-59=$ mildly atypical; 60-64 = moderately atypical; $\geq 65=$ markedly atypical). For hit reaction time, $T$-scores below 40 represent atypically fast reaction times. Thus, a response profile of high $T$-scores for commission errors, low $T$-scores for omission errors, and low $T$-scores for hit reaction time indicates impulsive performance.

\subsubsection{CPT reliability}

The split-half reliabilities are high for commission errors (.83) and hit reaction time (.95). With an interval of approximately 3 months between testing, the test-retest correlations were "highly satisfactory" for commission errors (.65) and hit reaction time (.55; CPT Manual; Conners, 2002, p.57). Little to no practice effects are observed for the CPT (Conners, 2002). The CPT has good discriminant validity, differentiating clinical (e.g., ADHD, patients with neurological damage), from non-clinical groups (Conners, 2002).

\subsubsection{Stop Signal Task (Verbruggen, Logan \& Stevens, 2008)}

The Stop Signal task is a two-choice reaction task. Participants are presented with a fixation point (a white cross in the centre of a black screen) followed by the presentation of either a white square or circle. As quickly and accurately as possible, 
participants press a left-hand response button (allocated to the " $z$ " key) when presented with a white square, or a right-hand response button (allocated to the "/" key) when presented with a white circle. The stimulus remains on-screen until a response is made or until 1250ms (maximum reaction time) lapses. Unexpectedly, on $25 \%$ of the trials an auditory tone $(750 \mathrm{~Hz}, 75 \mathrm{~ms})$ occurs after the presentation of the shape signalling that participants must inhibit the 'go' response. For no-signal trials, the default inter-stimulus interval is $2000 \mathrm{~ms}$, which is independent of 'go' reaction time. However, on stop-signal trials an auditory stop signal is presented after a variable stop signal delay (SSD). Stop signal delay is the time between presentation of the 'go' stimuli and the onset of the stop signal. Varying the SSD allows examiners to establish the maximum length of delay at which participants' response inhibition is still successful. The delay is initially set at $250 \mathrm{~ms}$ and is adjusted based on a method known as the "staircase tracking procedure" (Verbruggen, Logan \& Stevens, 2008, p.481). The staircase tracking procedure adjusts the SSD to achieve a probability of successful inhibition approximately $50 \%$ of the time. When inhibition is successful, the SSD increases by $50 \mathrm{~ms}$ but if inhibition fails, the SSD decreases by $50 \mathrm{~ms}$. Therefore, an SSD of $0 \mathrm{~ms}$ represents continually failed inhibition, whilst an SSD $>250$ represents successful inhibition. Greater SSD reflects faster implementation of inhibitory control.

Following 32 practice trials, the Stop Signal task has an experimental phase of three blocks of 64 trials each with a 10 second break in between blocks. During the break, participants receive on-screen feedback about their performance in the last block (the number of incorrect responses on the no-signal trials, the number of missed responses on no-signal trials, the mean reaction time on no-signal trials, and the percentage of correctly 
inhibited responses; Verbruggen, Logan \& Stevens, 2008). The average completion time for the task is 15 minutes.

\subsubsection{Stop signal outcome scores}

Speed of the inhibition process is an unobservable process, which must be estimated by other performance parameters. The Stop Signal Reaction Time (SSRT) is the primary outcome score used to interpret Stop Signal performance providing an estimate of the speed of inhibition (see Logan, 1994). SSRT is based on the race model of inhibition (Logan, 1994; Logan \& Cowan, 1984), which assumes that 'go' and 'stop' processes are independent. That is, when the stop signal forces inhibition of the ' $\mathrm{go}$ ' response, a race begins between response execution and inhibition processes. Whichever process finishes the race first determines success or failure of response inhibition.

The estimated time taken to inhibit a response is based on the distribution of ' $\mathrm{go}$ ' reaction times and the SSD. Thus, SSRT is calculated by:

\section{Mean no-signal reaction time-Mean $S S D^{9}$}

Estimating SSRT using this formula relies on the criterion that participants successfully inhibit their responses about $50 \%$ of the time. Although all 77 pre-treatment participants completed the Stop Signal task, data from 12 participants was removed due to variability in responding, and thus failure to meet the $50 \%$ inhibition criterion.

All outcome scores are automatically calculated as part of the Stop Signal software (Verbruggen, Logan \& Stevens, 2008). Outcome scores include: SSRT, mean probability of responding on a signal trial, mean SSD, mean reaction time when

\footnotetext{
${ }^{9}$ The mean stop signal delay at which approximately $50 \%$ of trials are inhibited. A threshold of $50 \%$ inhibition is the point at which performance data is reliable enough to estimate SSRT from.
} 
responding in error on stop-signal trials, mean reaction time on no-signal trials, percentage of correct responses on no-signal trials, and the percentage of missed responses on no-signal trials (Verbruggen, Logan \& Stevens, 2008).

\subsubsection{Stop signal reliability}

The normal range of SSRTs is between 200-250ms (Chambers, Garavan \& Bellgrove, 2009; F. Verbruggen, personal communication, May 19, 2009). That is, participants typically require the stop-signal to be presented $200-250 \mathrm{~ms}$ before their mean reaction time in order to cancel the response on $50 \%$ of the trials (Chambers et al., 2009, p.632). SSRTs greater than $250 \mathrm{~ms}$ indicate that people require an atypically longer period of time to successfully implement the inhibitory process.

Atypically long SSRTs have been shown to vary as a function of impulsivity (Logan, Schachar \& Tannock, 1997), and to reliably distinguish samples with impaired inhibitory control from controls (Lijffijt et al., 2005). The staircase tracking procedure is

considered a reliable method for estimating SSRT (Logan et al., 1997; Williams, Ponesse, Schachar, Logan \& Tannock, 1999).

\subsubsection{The Wechsler Abbreviated Scale of Intelligence (WASI; Wechsler, 1999)}

The WASI - a four-subtest version of the WAIS-III— produces an estimate of general intellectual ability, with higher scores indicating greater intellectual ability. The test is standardised and normed on a sample of 1145 adults $(M=50, S D=10)$. The four subtests include: Vocabulary, Block Design, Similarities and Matrix Reasoning. Scores from the four subtests are used to estimate Verbal, Performance and Full-scale IQ. Alternatively, Full-scale IQ can be estimated from a two-subtest form that utilises Vocabulary and Matrix Reasoning scores only. However, the two-subtest form does not 
allow for estimation of Verbal and Performance IQ. For the purpose of this thesis, scores from the two-subtest form were used to estimate Full-scale IQ; during the pre-treatment phase of data collection, the shorter form was used to keep the research session as short as possible to reduce participants' mental fatigue.

The Vocabulary subtest measures verbal knowledge, as participants are required to define words that are orally presented. The Matrix Reasoning subtest measures nonverbal fluid reasoning by presenting participants with an abstract, gridded pattern with a missing portion. From five choices of patterned squares, participants must choose the correct pattern to complete the overall gridded pattern.

The WASI two sub-test form is administered in approximately 15 minutes and was administered pre-treatment only. The average reliability for the four subtests ranges from .92-.94. Reliability coefficients are .96 for Verbal and Performance IQ and .98 for Full-scale IQ. The average reliability coefficient for the two subtests Full-scale IQ is .96 (Spreen \& Strauss, 1998).

\subsubsection{Violence Risk Scale (VRS; Wong \& Gordon, 2000)}

The VRS is a violence risk assessment tool based on 6 static risk items and 20 dynamic risk items. All items are derived from an extensive review of variables that are theoretically and empirically linked to violence. Each static or dynamic item is rated on a 4-point scale $(0,1,2$ or 3$)$. Items scored 0 represent an individual's area of strength whereas items scored 1 indicates that some features of the risk item are present but not to a significant degree. Items scored 2 or 3 represent a treatment target; these are risk factors that significantly contribute to an individual's violence risk over their lifetime functioning (Wong \& Gordon, 2000). 
With the exception of item 20 (current security level), all dynamic items rated a 2 or 3 also receive a stage of change rating. Stage of change ratings are based on the Transtheoretical Model of Change (Prochaska, DeClemente \& Norcross, 1992), which posits that individuals move through different levels of awareness and behavioural change during the change process: Pre-contemplation (no awareness of a problem or intention to change), Contemplation (awareness of a problem but no behavioural change evident), Preparation (behavioural changes evident but with recent lapses), Action (consistent behavioural changes evident), and Maintenance (sustained behavioural change over significant time periods and settings). Rating stage of change both before and after treatment allows practitioners to gauge the amount of change an individual has made on those items considered significant risk factors for violence. A validation study of 918 Canadian male federal offenders supports the reliability and validity of the VRS as a risk assessment instrument for both violent and general offending (see Wong \& Gordon, $2006)^{10}$.

The STURP facilitators completed VRS ratings pre and post-treatment. For 56 pre-treatment participants with a pre-treatment VRS, mean ratings from two static items supported that the majority of the sample had early-onset antisocial behaviour, consistent with Moffitt’s (1993) LCP typology. Item S4 assesses violence throughout the lifespan, and item S6 assesses the stability of family upbringing: higher ratings for each item indicate greater violence perpetrated and greater instability of family upbringing

\footnotetext{
${ }^{10}$ A sex offender version of the VRS (VRS-SO) was used for pre and post-treatment assessment of 15 participants in this thesis incarcerated for a sexual offence against adults. The VRS-SO is organised in the same format as the VRS but with the inclusion of risk factors theoretically and empirically related to sexual violence. Like the VRS, the VRS-SO has good psychometric properties (Wong \& Olver, 2010).
} 
characterised by exposure to antisocial and violent modeling (Wong \& Gordon, 2000). The mean for each item was close to the maximum score of 3: item $\mathrm{S} 4, M=2.80(S D=$ $.81)$; item $\mathrm{S} 6, M=2.55(S D=.81)$.

\subsection{Procedure ${ }^{11}$}

Treatment facilitators from each STURP group running during the recruitment period were informed about the research. If possible, facilitators briefed their group members about the research and the potential that they would be approached for participation. I then individually took each offender who agreed to hear more about the research to a quiet interview room within the therapy unit and gave him an information sheet outlining the objectives of the research and what participation would involve (see Appendices 1 and 2 for a copy of the pre-treatment information and consent forms). No one else was present during the research data collection session.

Offenders could choose to read the information sheet themselves or have it read to them. The independence of the research project from the Department of Corrections was emphasised and offenders were reassured that their participation would have no bearing on their sentence or treatment within the unit. Time was given for offenders to voice any questions or concerns they had about participation before deciding whether they would like to proceed. Offenders who declined participation returned to their daily activities. All consenting offenders signed two consent forms (one for the researcher and one that was placed on their institutional file), and remained for the testing session where they completed the IGT, CWIT, CTMT, CPT and Stop Signal tasks.

To promote a positive attitude to completing the research, I emphasised that the session would involve fun, but sometimes challenging, games and that the participant was

\footnotetext{
${ }^{11}$ Identical procedures were followed for post-treatment research data collection.
} 
not expected to get everything right, but just asked to do his best. The IGT, CWIT, CTMT, CPT and Stop Signal task were administered in one of five different orders randomly allocated to each participant to minimise the effects of order on the data. Before each new task, thorough instructions were given to participants as per the standard administration instructions for each task. The testing session took approximately 90 minutes (including the information process) with time for breaks when necessary.

Upon completion of the test battery, participants were given another opportunity to voice any questions or concerns about their participation. Participants were offered three small chocolate bars as a token of appreciation for their time and effort, which were consumed before returning to their cell ${ }^{12}$. Where available, the VRS, demographic and IQ data were extracted for each offender who participated in the research from electronic, programme-related databases.

\footnotetext{
12 The New Zealand Department of Corrections fully approved the offer of chocolate to participants as a token of appreciation for their time. The use of incentives for research participation with incarcerated offenders is an ethical practice (Hanson, Letourneau, Olver, Wilson \& Miner, 2012).
} 


\section{Chapter 5}

\section{Is cognitive impulse control intact or impaired in a sample of high-risk offenders?}

\subsection{Introduction}

Psychological reports, prison and probation-based case notes for high-risk offenders often include examples of criminal and non-criminal behaviour that appear to be the product of failed impulse control. According to Moffitt's (1993) developmental taxonomy, high-risk, LCP offenders are prone to impulsive behaviour because they experience biological and socialisation disturbances during development. In addition to effects on other important cognitive abilities, these disturbances impair the normal development of neural mechanisms that support impulse control. The proposed cognitive deficits influencing impulse control represent one risk factor maintaining the risk of antisocial behaviour throughout the life course.

As outlined in Chapter 2, studies of impulse control in adult offender samples typically utilise personality and behaviourally based assessment methods. When personality and behaviour suggest that an offender typically acts without forethought, it is inferred that the offender has an underlying weakness in the cognitive processes that support impulse control (e.g., acting before all relevant information was processed). However, as proposed in Chapter 2, empirical support for the validity of this inference is limited (Farrington, 2011). Moreover, error is easily introduced to the process of inferring cognition from naturalistic behaviour as explanations for dysfunctional behaviour unrelated to impaired cognitive impulse control are difficult to exclude without assessing cognition directly. The difficulties of achieving this differentiation are illustrated by the 
following hypothetical example: consider an assessor who uses a prison incident report as behavioural evidence to inform judgments about impulsivity. The incident report describes how the offender undergoing assessment physically assaulted another inmate in an ostensibly spontaneous attack. The offender was subsequently charged with assault. Should the risk assessor conclude that an assault charge was an undesirable outcome; therefore the offender acted on impulse by failing to consider the consequences of his behaviour? Alternatively, could the risk assessor also conclude that a possible assault charge was a risk the offender decided he was willing to take because he has learned that aggression wins respect from fellow offenders? (Walters, 2000). Did the offender's aggression look impulsive simply because it represented a well rehearsed, quickly selected product of many enactments of deliberate, goal-driven behaviour? (Aarts \& Dijksterhuis, 2000; Ouelette \& Wood, 1998).

There are important clinical implications in differentiating between the inabilities to resist an impulse and choice not to resist an impulse (Ogloff, 1997). Currently, in the context of the New Zealand Corrections system, common risk assessment methods (e.g., Violence Risk Scale) that include an impulsivity item capture impulsive-looking behaviour and personality style, but they cannot reliably inform whether cognitive impulse control impairments underlie behavioural and personality patterns; knowledge of which could greatly inform the risk assessment, clinical formulation and rehabilitation process.

As discussed in Chapter 2, we cannot reliably differentiate causal explanations for behaviour that are attributable to, versus distinct from, weaknesses in cognitive impulse control if impulse control is not assessed at the level of cognition. Because impulse 
control is a multidimensional construct, cognitive measures must capture the different domains of cognitive skills that support impulse control: Decision-making, perceptual and motor impulse control (Bechara, 2005). The assessment of distinct domains of cognitive impulse control provides a method for establishing unique patterns of impairment that can inform more tailored risk assessment and targeted treatment interventions.

\subsection{Study objectives and hypotheses}

Bechara suggests that within tightly controlled experimental settings it is possible to fractionate impairments in cognitive impulse control; that is, to isolate whether impairments differentially affect decision-making, perceptual or motor impulse control domains (Bechara, 2005). The first objective of this study is to establish whether principal components analysis supports Bechara's framework in a high-risk offender sample. Although the framework was developed from work with clinical populations that share non-criminal behavioural similarities to offenders, it is not yet clear whether the same cognitive impulse control dimensions can be distinguished in an offender sample. Specifically, I aim to empirically test whether a number of outcome variables obtained from the five tasks selected here to represent Bechara's three cognitive impulse control domains group together in a way that is conceptually consistent with Bechara's framework. Although Bechara's framework makes a case for three separate domains, it is also possible that the functions from each domain overlap in a normally functioning brain (Bechara, 2005). For example, to make a decision that takes both short and long-term consequences into account, perceptual impulse control functions are likely to be recruited to assist flexible consideration of all possible response options. Despite possible overlap, 
I based my predictions on the central idea that there are three potentially separable domains of cognitive impulse control that collectively assist the reflective system in behavioural control. I therefore hypothesised that principal components analysis of outcome scores from tasks representing each cognitive impulse control domain would produce a three-factor solution.

It is already well established that impulsive personality is a risk factor for general, sexual and violent offending (Andrews, Bonta \& Wormith, 2006; Wong \& Gordon, 2000). Consistent with Moffitt's assertion that impulsive personality is caused by weaknesses in cognitive impulse control, it follows that high-risk offenders should perform at a markedly more dysfunctional level on cognitive impulse control tests than non-offender, normative controls who are at low risk of offending. However, empirical tests of this notion are lacking (Farrington, 2011). Accordingly, the second objective is to establish what proportion of an adult, high-risk offender sample—suggested to predominantly represent offenders on the LCP-path—exhibit clinically significant impairment within, and across, different cognitive impulse control domains. Clinical impairment was chosen as the outcome criterion for three reasons: 1) to avoid overpathologising offender performance because it is common for cognitively intact, nonoffenders to score below average on at least one neuropsychological test within a battery including multiple tests (Binder, Iverson \& Brooks, 2009), 2) Moffitt's (1993) LCP theory suggests that cognitive impulse control is impaired to a dysfunctional degree in order to maintain criminal risk, and 3) it is expected that functioning substantially below that of the general population is the level at which functioning is most likely to become maladaptive (Silverberg \& Millis, 2009). 
The third objective is to establish whether specific domains of cognitive impulse control are clinically impaired; that is, whether impairment affects one, two or all three cognitive impulse control domains. Investigating patterns of impairment provides a greater level of specificity for formulations of impulsivity than can be achieved by personality and behavioural risk assessment measures alone.

Regarding the second and third objectives, my hypotheses are based on two general findings and one theoretical premise: 1) the majority (66\%) of New Zealand's high-risk offenders meet the early-onset criteria for the LCP typology (Wilson, 2004), 2) although findings are mixed, previous research suggests that adult offenders could be impaired in either decision-making, perceptual or motor impulse control domains (see Chapter 2), and 3) Moffitt's LCP theory predicts that poor impulse control is a trait level risk factor maintaining risk of antisocial behaviour throughout the life course. Accordingly, I hypothesised that clinically impaired cognitive impulse control would be prevalent in this adult, high-risk offender sample assumed to predominantly represent Moffitt's LCP type. It was also hypothesised that the impairments would affect all 3 cognitive impulse control domains.

\subsection{Results and discussion}

All data presented here are from the pre-treatment research assessment. Each cognitive impulse control task generated a range of outcomes scores (see Chapter 4 for a full description of each task). Some outcome scores capture functions that are important to understanding executive functioning globally, but are not of central importance to understanding performance across Bechara's domains. Therefore, the first step in evaluating whether performance was intact or impaired within or across domains was to 
conduct an exploratory principal component analysis (PCA) of the outcome scores across all three domains to establish empirical support for Bechara's framework. The selection of outcome scores for the PCA was guided directly by recommendations from each task's professional manual, or by conventions in previous research regarding the most reliable indicators of impulsive performance. Table 2 displays the 9 raw outcome scores selected for the exploratory principal components analysis (PCA).

Table 2. Outcome scores from each task within Bechara's three domains of cognitive impulse control
Domain
$\operatorname{Task}(\mathrm{s})$
Outcome score(s)

\section{Decision-making \\ Perceptual Impulse Control}

Motor Impulse Control

\section{D-KEFS Colour-word Interference (CWIT)}

Comprehensive Trail Making Test (CTMT)

Continuous Performance Test (CPT)

Stop signal
1. Block 2 net total

2. Block 5 net total

3. Inhibition completion time

4. Inhibition/switch completion time

5. Trail 4 completion time

6. Trail 5 completion time

7. Hit reaction time

8. Commission errors

9. Stop Signal reaction time (SSRT)

\subsubsection{Rationale for selection of outcome scores from each task}

The IGT was used to assess decision-making. As outlined in Chapter 4, total net scores provide a crude indication of performance but they do not differentiate choices 
made early in the task when choices are made under conditions of ambiguity—because participants are still exploring reward and punishment contingencies—-from choices made later in the task when deck contingencies become more obvious and therefore represent choice under conditions of risk (Buelow \& Suhr, 2009; Krain, Wilson, Arbuckle, Castellanos \& Milham, 2006). Block 2 and 5 net scores were selected to provide a 'snapshot' of performance at early and later stages in the task. If Block 2 and 5 net scores load on the same factor, this would indicate that performance in early and later blocks is similar and may not rely on different cognitive processes; thus, total net scores could be used as the primary performance outcome for the IGT. If Block 2 and 5 net scores load on different factors, the use of total net scores as the primary performance outcome may not be suitable to accurately represent overall performance.

The D-KEFS CWIT and the CTMT assessed performance in the perceptual impulse control domain. Both tasks assess baseline abilities (e.g., word reading in the CWIT or visual scanning in the CTMT), before assessment of higher-order skills. Outcome scores from baseline conditions assist test interpretation by isolating baseline impairment from impairment in higher-order skills only. However, the baseline outcome scores themselves are not central to Bechara's framework. Therefore, only the higherorder outcome scores for the inhibition and inhibition/switching conditions in each task were included in the PCA to represent the perceptual impulse control domain.

The CPT and the Stop Signal task assessed performance in the motor impulse control domain. Hit reaction time and commission errors were selected for the PCA for the CPT as per the manual's recommendation that fast hit reaction times and a high rate of commission errors indicate impulsive performance (CPT Manual; Conners, 2002, 
p.29). The stop signal reaction time (SSRT) was the primary outcome variable selected from the Stop Signal task because it provides a reliable estimate of inhibitory performance (Verbruggen, Logan, \& Stevens, 2008).

\subsubsection{Principal components analysis (PCA) results}

The factorability of scores was examined. The correlation matrix had a number of coefficients of .30 and above. An examination of the Kaiser-Meyer-Oklin measure of sampling adequacy suggested that the sample was factorable $(\mathrm{KMO}=.563$ exceeding the recommended value of .5; Field, 2009). In addition, Bartlett's Test of Sphericity reached statistical significance $\left(\chi^{2}([36]=113.89, p<.01)\right.$.

Given these overall indicators, a Principal Components Analysis with Varimax (orthogonal) rotation was conducted on the 9 outcome scores. Three components emerged with eigenvalues over 1, explaining $26.53 \%, 20.25 \%$ and $15.75 \%$ of the variance, respectively. Examination of the scree plot showed a classic "elbow" after three factors. Table 3 displays the rotated factor loadings of each outcome score. Factor loadings above .32 are in boldface consistent with Tabachnik and Fidell's (2001) recommendation for minimum factor loadings. 
Table 3. Rotated factor loadings for 9 outcome scores selected for each cognitive impulse control domain

\begin{tabular}{lccc}
\hline & \multicolumn{3}{c}{ Factor Loadings } \\
\cline { 2 - 4 } Outcome scores & 1 & 2 & 3 \\
IGT Block 2 Net & -.065 & .082 & $\mathbf{. 8 2 3}$ \\
IGT Block 5 Net & .027 & -.016 & $\mathbf{. 7 9 4}$ \\
CWIT: Inhibition RT & $\mathbf{. 7 0 1}$ & $\mathbf{. 3 4 9}$ & -.209 \\
CWIT: Inhibition/switch RT & $\mathbf{. 4 6 1}$ & $\mathbf{. 6 2 0}$ & -.068 \\
CTMT: Trail 4 RT & $\mathbf{. 8 3 5}$ & -.081 & .108 \\
CTMT: Trail 5 RT & $\mathbf{. 7 9 5}$ & -.024 & .031 \\
CPT: Hit RT & -.161 & $\mathbf{. 8 9 0}$ & -.154 \\
CPT: Commission errors & $\mathbf{. 5 4 6}$ & $\mathbf{- . 5 5 4}$ & -.151 \\
SSRT & .064 & $\mathbf{. 4 4 6}$ & .167 \\
\hline
\end{tabular}

Note. IGT = Iowa Gambling Task; CWIT $=$ Colour-Word Interference Test; RT = Response/Reaction Time; CTMT $=$ Comprehensive Trail-Making Test; $\mathrm{CPT}=$ Continuous Performance Test; SSRT = Stop Signal Reaction Time.

The first point to note from Table 3 are the cross-loadings $>.32$ shared between Factor 1 and 2, but not Factor 3. The outcome scores loading exclusively on Factor 3 are derived from the IGT, suggesting similar performance in early (block 2) and later stages (block 5) of the IGT. Decision-making ability at early and late stages was not related to abilities from the perceptual or motor impulse control domain. Accordingly, Factor 3 was labeled Decision-making.

Factor 1 and 2 represent outcomes from tasks within the perceptual and motor impulse control domains. Specifically, four out of five outcomes loading on Factor 1 are from the CWIT and CTMT: both are perceptual impulse control tasks capturing 
inhibition and set shifting ability. The fifth outcome loading on Factor 1 is the number of commission errors made on the CPT: that is, erroneous responses to the non-target letter $\mathrm{X}$. Although the CPT was selected to assess motor impulse control, the pattern of loadings on Factor 1 suggests that this task draws on functions that overlap with perceptual impulse control skills: inhibition and set shifting. Participants must adhere to two response rules on the CPT for accurate performance: 1) respond to all non-X letters; 2) inhibit responses to the letter X. Arguably, flexible shifting between response rules is necessary to provide the correct response, which might account for the cross-loading of CPT commission errors with inhibition and set shifting outcomes (Schuch \& Koch, 2003). Accordingly, Factor 1 was labeled Perceptual Impulse Control. Exclusive loading on this factor by CTMT Trail 4 and 5 suggests that these outcomes may provide a more pure indication of perceptual impulse control than inhibition and set shifting on the CWIT.

For the most part, the pattern of factor loadings on Factor 2 consists of outcomes from motor impulse control tasks (CPT Hit RT, CPT commission errors and SSRT). Accordingly, Factor 2 was labeled Motor Impulse Control. However the cross-loadings from inhibition and set shifting on the CWIT again suggests that perceptual and motor impulse control draw on similar processes. Although SSRT from the Stop Signal task had a relatively smaller loading than the CPT outcomes on this factor, SSRT did not crossload on any other factors. Thus, consistent with previous research, the SSRT appeared to provide the most pure indication of response inhibition in the motor impulse control domain (Nigg, 2000). 


\subsubsection{PCA summary}

The PCA results provided empirical support for Bechara's framework; particularly regarding dissociations between decision-making performance in the gambling task and other executive abilities (Bechara et al., 2001; Brand, Recknor, Grabenhorst \& Bechara, 2007; Shuster \& Toplak, 2009; Stocco, Fum, \& Napoli, 2009; Toplak, Sorge, Benoit, West \& Stanovich, 2010). Consistent with Bechara's assertion of overlapping functions across domains, perceptual and motor impulse control tasks appeared to draw on similar processes if the unique requirements of the tasks were such that inhibition and cognitive flexibility were required for fast, accurate responding.

\subsubsection{Exploring clinically impaired performance}

To evaluate clinically impaired performance, raw outcome scores were converted to $T$-scores and percentiles (where available). Where available, for each independent task, $T$-score thresholds for impaired performance listed in the tasks' professional manuals were used. For tasks that did not provide a threshold for impaired for performance, the common neuropsychological convention of scores at least 2 SDs below the normative mean was used as the impairment threshold (Schretlen, Testa, Winicki, Pearlson \& Gordon, 2008). 


\subsubsection{Decision-making performance}

There are different conventions — some more liberal than others-for oeprationalising impaired performance on the IGT. In the present study, impaired performance was based on stringent criteria determined by demographically-corrected $T$ score guidelines (IGT Manual; Bechara, 2007). The guidelines are: $T \leq 39=$ impaired range, $T$ 40-44 = below average range, $T \geq 45=$ non-impaired range (p.8). Therefore, $T$ scores 39 or below represented impaired performance. A $T$-score $\leq 39$ means that participants are performing at a low decision-making capacity within the range of neurologically impaired patients (IGT Manual; Bechara, 2007, p.8). Table 4 displays mean raw total and block net scores, with corresponding $T$-scores and percentile ranks.

Table 4. IGT raw total and block net scores with normative comparisons $(N=77)$

\begin{tabular}{lcccc}
\hline $\begin{array}{c}\text { IGT outcome } \\
\text { scores }\end{array}$ & $\begin{array}{c}\mathrm{M}(S D) \\
\text { raw score }\end{array}$ & $\begin{array}{c}95 \% \mathrm{CI} \\
\text { raw score }\end{array}$ & $\begin{array}{c}\mathrm{M}(S D) \\
T \text {-score }\end{array}$ & \% ile rank \\
\hline Net Total & $5.90(24.76)$ & {$[.28,11.52]$} & $47.88(8.27)$ & 42 \\
Block 1 (1-20) & $-.81(5.90)$ & {$[-2.14, .53]$} & $54.56(8.83)$ & 69 \\
Block 2 (21-40) & $1.09(6.91)$ & {$[-.48,2.66]$} & $47.51(8.53)$ & 42 \\
Block 3 (41-60) & $2.75(7.52)$ & {$[1.05,4.46]$} & $48.64(7.72)$ & 42 \\
Block 4 (61-80) & $1.45(7.93)$ & {$[-3.5,3.25]$} & $46.97(7.85)$ & 38 \\
Block 5 (81-100) & $1.40(8.25)$ & {$[-.47,3.27]$} & $46.61(9.22)$ & 34 \\
\hline
\end{tabular}

Note. $T$-score $M=50, S D=10 ; \mathrm{CI}=$ Confidence Interval.

As Table 4 shows, with the exception of Block 1, raw total and block net scores were positive with corresponding $T$-scores above 45 , thus indicating nonimpairment overall. Although block 1 mean raw net scores were negative indicating 
disadvantageous choices in the first 20 card selections, as the $T$-score of 54 indicates, this pattern is normative; participants are still at the stage of exploring reward and punishment contingencies in each deck this early in the task (Brand, Recknor, Grabenhorst \& Bechara, 2007). Although mean scores indicated that performance was non-impaired, closer inspection of the direction of scores suggests that performance did not necessarily fit the optimal choice pattern either based on Bechara's (2007) criteria that healthy performance is indicated by a pattern of gradually learning to choose roughly the same number of cards from good decks (i.e., C and D), whilst avoiding selections from bad decks (i.e., A and B). This pattern is reflected by block net scores that develop from around zero at the beginning of the task toward a positive value in favour of more advantageous selections at later stages of the task (Hortsmann, Villringer \& Neumann, 2012). Table 4 shows that although mean block net scores were not within the clinically impaired range, they generally increased to reach their most advantageous peak in block 3 , but then decreased in blocks 4 and 5 . This quadratic function is very different to the positive linear function Bechara predicts for healthy performance. Following analysis of proportions of the sample with performance in the clinically impaired range across each block, a pattern of increasingly disadvantageous performance as the task progressed became even more prominent (Table 5). 
Table 5. Proportion of participants with net scores in the clinically impaired range

\begin{tabular}{lc}
\hline \multicolumn{1}{c}{ IGT outcome scores } & $\begin{array}{c}\text { Proportion impaired net } \\
\text { scores }(T \leq 39)\end{array}$ \\
\hline Total net score & $12.99 \%$ \\
Block 1 (1-20) & $2.60 \%$ \\
Block 2 (21-40) & $10.39 \%$ \\
Block 3 (41-60) & $11.69 \%$ \\
Block 4 (61-80) & $14.29 \%$ \\
Block 5 (81-100) & $19.48 \%$ \\
\hline
\end{tabular}

A simple count of participants with positive and negative total net scores revealed that nearly half of the sample had negative scores; indicating disadvantageous choices due to greater selections from decks with high reward but even higher losses. However, a much smaller proportion —approximately $13 \%$ —made sufficiently numerous disadvantageous choices to place their performance within the range of neurological patients, as indicated by total net $T$-scores $\leq 39$ (Table 5 ). That is, overall, $13 \%$ of the sample made similar choices in the gambling task as patients with VMPC damage, whose decision-making is characterised by neglect of future outcomes due to impaired ability to learn from previous mistakes. Based on Moffitt's (1993) LCP theory and the many anecdotal examples in which the real-life behaviours exhibited by high-risk offenders seem exclusively driven by immediate needs, it would be reasonable to expect a higher proportion of impairment than that found. However, as highlighted above, the performance of those found not to be clinically impaired was not necessarily normal by Bechara's standards. Perhaps the most revealing observation in Table 5 is the steady 
increase in the proportion of scores within the impaired range as the task progressed: a pattern in direct contrast to the improvements in learning of adaptive responses expected in healthy performance.

If only $13 \%$ of the sample performed within the clinically impaired range, how can we explain the anomalous observation that performance became more disadvantageous as the task progressed? To further disentangle choice patterns and investigate explanations for this anomalous observation, I adopted the recommendations of various authors to analyse performance at the level of deck selections across each block of 20 trials (e.g., Buelow \& Suhr, 2009; Hortsmann, Villringer \& Neumann, 2012; Lin, Chiu, Lee \& Hsieh, 2007). The mean number of selections made from each deck across 5 blocks is presented in Table 6 .

Table 6. Mean number of card selections from each deck per block of 20 cards

\begin{tabular}{lccccc}
\hline & Block 1 & Block 2 & Block 3 & Block 4 & Block 5 \\
\hline Deck A & 4.4 & 3.9 & 3.4 & 3.3 & 3.6 \\
Deck B & 6.0 & 5.3 & 5.2 & 5.9 & 5.7 \\
Deck C & 4.5 & 4.4 & 4.5 & 4.3 & 4.5 \\
Deck D & 5.1 & 6.4 & 6.9 & 6.4 & 6.2 \\
\hline
\end{tabular}

Each card deck differs not only in reward and loss outcome but also in reward and loss frequency. Regarding reward and loss outcome, Decks A and B are high-risk and disadvantageous because they return high reward but even higher losses; continual selections ultimately result in a net loss. Conversely, Decks C and D are advantageous and low risk because they return small reward but even smaller losses; continual 
selections ultimately result in a net win. Regarding reward and loss frequency, Decks A and $\mathrm{C}$ are disadvantageous because they have the lowest frequency gains and highest frequency losses (5 wins: 5 losses), whereas Decks B and D are advantageous because they have the highest frequency gains and lowest frequency losses (9 wins: 1 loss).

The mean number of deck selections across blocks displayed in Table 6 show that Deck A was uniformly chosen the least, followed by Deck C. With the exception of the first block, in which most choices were made from Deck B, Deck D was systematically preferred across all blocks followed closely by Deck B. Considering the pay-off schedules for each deck, the deck selection patterns suggest that participants primarily preferred decks with the lowest loss frequency, rather than Bechara's predictions for healthy performance: decks with the best long-term outcomes. Impaired performance is indicated by continued selections from disadvantageous decks — including Deck Bparticularly in the final blocks of the task, by which time participants should have learned to avoid bad decks. Therefore, although decision-making performance was partly 'healthy' as indicated by the Deck D preference, it was also partly 'unhealthy' relative to Bechara's criteria, because participants did not gradually come to avoid Deck B. This is likely to explain why the proportion of clinically impaired performance steadily increased throughout the task. In other words, participants' learning pattern showed a tendency to choose decks with the lowest likelihood of loss, regardless of the win rates for those decks.

Although continued preference for Deck B is abnormal according to Bechara's predictions for healthy performance, Deck B preference is normative according to an emerging body of literature. For example, Hortsmann, Villinger and Neumann (2012) 
found that neurologically healthy participants weighted gain and loss frequency above net outcomes when making decisions on the gambling task, ultimately making the most selections from Decks B and D (low loss frequency), and the least from Decks A and C (high loss frequency). They also found that healthy participants learned to distinguish between choices that lead to advantageous (Deck C) and disadvantageous (Deck A) net outcomes, as indicated by relatively more avoidance of Deck A than Deck C. However, healthy participants did not distinguish between good or bad net outcomes for Decks B and D. In contrast, in this study, participants $d i d$ choose according to both the best net outcomes and the lowest loss frequencies: from the low loss frequency decks (B and D), Deck D (advantageous net outcome) was favoured over Deck B (disadvantageous net outcome); and from the high loss frequency decks (A and C), Deck C (advantageous net outcome) was favoured over Deck A (disadvantageous net outcome).

Taken together, choice patterns in the gambling task captured low preference for choices with high frequency punishment, and high preference for choices with good net outcomes. By Bechara's standards, this renders offender's performance partly disadvantageous because healthy performance is exclusively operationalised as gradual avoidance of decks with disadvantageous net outcomes (i.e., including avoiding Deck B). Therefore, clinical impairment—calculated with reference to a normative group with improving avoidance of disadvantageous decks — increased as the game progressed as participants maintained their Deck B preference (though it was not preferred as much as Deck D). However, Deck B preference is rendered advantageous and normal by the standards of emerging evidence suggesting that this choice pattern mimics that of normal, 
neurologically healthy participants (e.g., Singh \& Khan, 2009; Steingrover, Wetzels, Horstmann, Neumann, \& Wagenmakers, 2013).

\subsubsection{Perceptual impulse control performance: CWIT}

Raw completion times for each condition were converted to age-corrected scaled scores $(M=10, S D=3)$. A scaled score above the mean indicates good performance, whilst a score below the mean indicates below average or impaired performance. According to convention in the neuropsychological literature of 2 SDs below the normative mean reflecting impaired performance, clinical impairment on the CWIT was operationalised as any scaled score of 4 or below. Contrast scores were also calculated to establish equivalence or disproportionately different performance on baseline (colour naming, word reading) and higher-order conditions (inhibition, inhibition/switching). For each condition, mean raw completion times, mean completion time scaled scores, and mean contrast scaled scores are displayed in Table 7. 
Table 7. CWIT mean raw completion time, scaled and contrast scores $(N=74)$

\begin{tabular}{|c|c|c|c|}
\hline CWIT outcome scores & $\begin{array}{c}\mathrm{M}(S D) \\
\text { Raw completion } \\
\text { time }\end{array}$ & $\begin{array}{c}95 \% \mathrm{CI} \\
\text { Raw completion } \\
\text { time }\end{array}$ & $\begin{array}{c}\text { Scaled score } \\
(M=10, S D=3)\end{array}$ \\
\hline Colour naming & $29.57(5.17)$ & {$[28.38,30.76]$} & $9.21(2.34)$ \\
\hline Word reading & $22.74(4.07)$ & {$[21.81,23.68]$} & $9.54(2.45)$ \\
\hline Inhibition & $57.56(16.09)$ & {$[53.86,61.26]$} & $9.04(2.77)$ \\
\hline Inhibition/switching & $75.88(20.64)$ & {$[71.13,80.63]$} & $6.83(3.38)$ \\
\hline $\begin{array}{l}\text { Combined Naming }+ \text { Word } \\
\text { Reading }\end{array}$ & - & - & $9.69(2.21)$ \\
\hline Contrast $1^{\mathrm{a}}$ & - & - & $9.75(2.50)$ \\
\hline Contrast $2^{b}$ & - & - & $7.15(2.78)$ \\
\hline Contrast $3^{\mathrm{c}}$ & - & - & $7.60(3.31)$ \\
\hline
\end{tabular}

Note. ${ }^{\mathrm{a}}$ Inhibition vs. colour naming; ${ }^{\mathrm{b}}$ Inhibition/switching vs. combined word read and colour naming; ${ }^{\mathrm{c}}$ Inhibition vs. Inhibition/switching

The scaled scores in Table 7 show that completion times for the baseline conditions — colour naming and word reading — were slightly below average but still within $1 \mathrm{SD}$ of the normative mean of 10 . That is, participants were fluent in their ability to name high-frequency words (colours) and read high-frequency words. The scaled score for the inhibition condition was also lower than the normative mean but still within $1 \mathrm{SD}$. The inhibition condition requires inhibition of the habitual response to read a word, to name the incongruent ink colour the word is printed in instead. Therefore, the equivalence of scaled scores in the colour naming and inhibition conditions suggests that 
the group did not display a deficit in verbal inhibition over and above their colour naming ability. This equivalence is supported by a mean Contrast 1 score of 9.75: contrast scaled scores between 8-12 represent equivalent performance in each condition (D-KEFS Manual; Delis, Kaplan, \& Kramer, 2001, p.99).

The poorest performance was observed for the inhibition/switching condition with a mean scaled score of 6.8 , falling more than $1 \mathrm{SD}$ below the normative mean of 10 . If performance is adequate on all previous conditions (i.e., colour naming speed, reading speed and inhibition), but falters on inhibition/switching, the dysfunction is most likely in cognitive flexibility, or the ability to flexibly set shift between perceptual sets (D-KEFS Manual; Delis, Kaplan, \& Kramer, 2001, p.103). The pattern of scores in Table 7 supports an isolated deficit in inhibition/switching relative to previous conditions. Contrast 2 and 3 scores also support a disproportionate deficit in cognitive flexibility compared to baseline and basic inhibition ability (contrast scores $\leq 7$ represent disproportionately worse performance on the higher-order task relative to that on the baseline measure; D-KEFS Manual; Delis, Kaplan, \& Kramer, 2001, p.99).

Error rates for each condition were assessed to rule out the possibility that normal completion times were simply a function of high error rates. Colour naming and wordreading errors were normal; $53 \%$ and $58 \%$ of the normative sample made a similar or greater number of errors, respectively. Naturally the error rates were higher in the more difficult conditions of inhibition (mean error scaled score $=7.9$ ) and inhibition/switching (mean error scaled score $=8.2$ ). However, both scaled scores were within $1 \mathrm{SD}$ below the normative mean indicating below average, but not clinically impaired error rates. 
To establish what proportion of the sample displayed clinically impaired inhibition and inhibition/switching relative to baseline abilities, I identified how many participants had normal baseline abilities, but clinically impaired scaled scores for the inhibition and inhibition/switching conditions (scaled score $\leq 4$ ). Only 1 participant displayed clinically impaired inhibition relative to their baseline performance. However, 18/75 (24\%) participants displayed clinically impaired inhibition/switching abilities relative to their performance on either the baseline or inhibition conditions. Thus, approximately one quarter of the sample was exclusively clinically impaired in cognitive flexibility, as assessed by the CWIT.

To summarise, for the majority of the sample, completion times and error rates were below average, but largely inconsistent with a clinically impulsive profile; indicated by atypically fast completion times and high error rates. Elevated error rates in the inhibition and inhibition/switching conditions appeared to be made purely because of the difficulty of these conditions, not because participants were trading speed for accuracy. Importantly, without prompting, participants corrected the majority of errors in the inhibition and inhibition/switching conditions. Put another way, they displayed the capacity to monitor, and correct their own incorrect responses, which is incongruent with deficits in perceptual impulse control (D-KEFS Manual; Delis, Kaplan \& Kramer, 2001 p.103). For a smaller proportion of the group, perceptual impulse control was an area of significant cognitive weakness, but it was specifically limited to cognitive flexibility and did not extend to basic inhibition of an over learned, habitual thought. 


\subsubsection{Perceptual impulse control performance: CTMT}

On the CTMT, raw completion times for all 5 trails are converted to age-corrected $T$-scores $(M=50, S D=10)$. Individual trail $T$-scores are pooled to provide the Composite Index; a general performance indicator. $T$-scores for individual trails and the Composite Index are interpreted as follows: $>70$ Very superior; 65-70 Superior; 58-64 High Average; 43-57 Average; 36-42 Below Average; 30-35 Mildly to Moderately Impaired; < 30 Severely Impaired (CTMT Manual; Reynolds, 2002, p. 14). As per the recommendations in the CTMT manual, a $T$-score $\geq 1.5$ SDs below the mean (i.e., $T \leq 35$ ) was representative of clinically impaired performance (CTMT Manual; Reynolds, 2002, p. 14). Table 8 displays raw completion times (measured in seconds), $T$-scores and percentile ranks for individual trails and the Composite Index.

Table 8. CTMT mean raw completion time and normative comparisons $(N=77)$

\begin{tabular}{ccccc}
\hline $\begin{array}{c}\text { CTMT } \\
\text { outcomes }\end{array}$ & $\begin{array}{c}\mathrm{M}(S D) \\
\text { Raw Score }\end{array}$ & $95 \% \mathrm{CI}$ & $\begin{array}{c}\mathrm{M}(S D) \\
\text {-score } \\
(M=50, S D=10)\end{array}$ & \%ile rank \\
\hline Trail 1 & $34.79(11.03)$ & {$[32.29,37.30]$} & $46.71(10.74)$ & 40 \\
Trail 2 & $30.53(9.95)$ & {$[28.27,32.79]$} & $54.61(13.53)$ & 59 \\
Trail 3 & $32.49(12.13)$ & {$[32.49,38.00]$} & $49.61(9.58)$ & 50 \\
Trail 4 & $34.03(14.70)$ & {$[30.69,37.36]$} & $46.10(11.83)$ & 39 \\
Trail 5 & $60.65(22.63)$ & {$[55.51,65.79)$} & $42.09(9.93)$ & 28 \\
Composite & - & - & $47.13(9.53)$ & 42 \\
Index & - & - & & \\
\hline
\end{tabular}

In Table 8, the mean Composite Index $T$-value of 47.13 shows that overall performance was within the average range. The percentile rank indicates that $42 \%$ of the 
normative population scored at or below this level of performance. Inspection of each individual trail score shows that the sample performed within an average range for all but Trail 5-the most difficult trail requiring shifting between two different perceptual setswhich sat on the cut-off for below average performance. Only $28 \%$ of the normative sample took as long, or longer to complete Trail 5.

To isolate the proportion of participants who performed normally on Trails 1-3, but at a clinically impaired level on Trails 4-5, I selected all participants with a $T$-score $\leq$ 35 on each trail then removed data from any participants who had impairment on Trails 4-5 as well as impairment on Trails 1-3. This pattern of impairment indicates that difficulty on Trails 4-5 could be accounted for by difficulty in baseline skills (e.g., visual scanning), rather than cognitive flexibility per se. For Trail 4, 11/77 (14.29\%) performed within the mildly to moderately impaired, or severely impaired range. For Trail 5, 16/77 (20.78\%) performed within the mildly to moderately impaired, or severely impaired range. Ten participants (13\%) were clinically impaired on both Trails 4 and 5 relative to their performance on simple trails. Taken together, no more than one fifth of the sample produced scores indicative of mild to moderate or severe impairment on either Trail 4 or 5 compared to performance on simple trails. A relatively smaller proportion of the sample (13\%) was clinically impaired on both Trails 4 and 5 compared to performance on simple trails.

\subsubsection{Motor impulse control performance: $C P T$}

All raw scores are converted to age-corrected $T$-scores $(M=50, S D=10)$. For the majority of outcome variables, high $T$-scores and percentiles are indicative of impaired performance: $T \geq 65=$ markedly atypical; $T$ 60-64 = moderately atypical (CPT Manual; 
Conners, 2002, p.23). However, for hit reaction time and Beta, lower $T$-scores can reflect more impulsive performance. For hit reaction time, a low $T$-score represents fast reaction times: when paired with a high $T$-score for commission errors, and a low $T$-score for omission errors, this pattern reflects impulsive performance. Similarly, for Beta, lower values reflect a response style that favours response speed over accuracy. For hit reaction time and Beta, $T$-scores 40-36 are moderately atypical and $T$-scores $\leq 35$ are markedly atypical. Descriptive statistics and normative comparisons for indicators of general and impulsivity-specific performance on the CPT are displayed in Table 9.

Table 9. CPT mean raw scores and normative comparisons $(N=74)$

\begin{tabular}{|c|c|c|c|c|}
\hline CPT outcomes & $\begin{array}{c}\mathrm{M}(S D) \\
\text { Raw score }\end{array}$ & $\begin{array}{c}95 \% \text { CI } \\
\text { Raw score }\end{array}$ & $\begin{array}{l}\mathrm{M}(S D) \\
T \text {-score }\end{array}$ & $\begin{array}{l}\text { \%ile } \\
\text { rank }\end{array}$ \\
\hline$d$-prime & $.53(.39)$ & {$[.43, .61]$} & $53.16(7.94)$ & 62 \\
\hline Hit RT ISI Change & $.05(.04)$ & {$[.04, .06]$} & $50.76(11.31)$ & 52 \\
\hline Hit SE ISI Change & $.01(.15)$ & $-[.03, .04]$ & $50.39(14.80)$ & 48 \\
\hline Beta & $.87(1.37)$ & {$[.55,1.19]$} & $50.29(10.20)$ & 48 \\
\hline $\begin{array}{l}\text { Hit RT Block } \\
\text { Change }\end{array}$ & $.02(.03)$ & {$[.01, .02]$} & $55.65(11.08)$ & 64 \\
\hline $\begin{array}{l}\text { Hit SE Block } \\
\text { Change }\end{array}$ & .03 (.09) & {$[.01, .05]$} & $56.61(13.37)$ & 63 \\
\hline Hit RT & $341.09(68.08)$ & {$[325.32,356.87]$} & $41.42(12.79)$ & 29 \\
\hline Commissions & $17.91(8.26)$ & {$[15.99,19.82]$} & $54.69(10.20)$ & 62 \\
\hline Omissions & 7.05 (12.07) & {$[4.26,9.85]$} & $63.40(37.18)$ & 62 \\
\hline
\end{tabular}

Note. $\mathrm{RT}=$ Reaction Time, $\mathrm{SE}=$ Standard Error, ISI = Inter-stimulus Interval. 


\subsection{General performance indicators}

$D$-prime represents sensitivity, or how well participants could discriminate between the target (non-X letters) and the non-target (letter X). Table 9 shows that the $d$ prime $T$-score is close to the normative mean suggesting that participants were not impaired in their ability to discriminate between the target and non-target. In addition, the inter-stimulus interval change statistics (Hit RT and SE ISI change) were not elevated: this means that participants were able to adjust their responses according to the different inter-stimulus intervals of 1,2 , or 4 seconds between the presentation of each letter.

Beta represents general response style. A cautious response style favours accuracy over speed (e.g., $T \geq 60$ ), while an impulsive response style favours speed over accuracy (e.g., $T \leq 40)$. The mean Beta $T$-value of 50 shows that participants had a normal response style and were not overly cautious nor overly inclined to trade speed for accuracy.

The Hit RT and Hit SE block change scores represent vigilance, or the ability to sustain attention over time. The $T$-scores for both indicators are within the mildly atypical range ( $T$ 55-59) indicating that reaction times slowed (Hit RT block change) and became more inconsistent (Hit SE block change) as the task progressed. Although not in the clinically impaired range, these indicators highlight the difficulty participants had in maintaining consistent responding as attention waned throughout the task.

\subsection{Impulsive performance indicators}

As stated above, an impulsive profile is indicated by fast hit reaction times, a high rate of commission errors but relatively fewer omission errors (CPT Manual; Conners, 2002, p.29). Commission errors are responses made to a non-target (letter X), whilst 
omission errors are responses withheld when a response was required (non-X letter). Table 9 shows that reaction times were faster than average with a mean $T$-score of 41 (low scores indicate fast responses). Although below the mean of 50, this $T$-score is still within $1 \mathrm{SD}$ from the normative mean. Therefore, reaction times were not atypically fast. The commission error rate was at the upper limit of the average range, but also not clinically impaired. A relatively larger error rate was observed for omission errors-in the moderately atypical range - relative to commission errors. A high rate of omission errors either means subjects were not orienting to the target or that they had very sluggish responses (CPT Manual; Conners, 2002, p.29). Because there was no other evidence to indicate sluggish responding, it is more likely that the moderately high omission rate was due to failure to orient to targets and maintain attention. Anecdotally, this possibility was supported by my observations during the task: many participants fidgeted, tried to engage in conversation with me and commented about the challenge of remaining focused for the full duration of the task. Difficulty with waning attention was also supported empirically with reference to the vigilance scores described above in section 5.3.3.4.1.

Although performance at a group level was not consistent with impulsivity, I calculated the proportion of participants who did exhibit clinically impaired performance by conducting simple counts of participants with impaired commission error rates ( $T \geq$ $60)$ and atypically fast hit reaction times $(T \leq 40)$. Of the 74 participants who completed the CPT, 19 (25.68\%) fit the impulsive profile with abnormally high raw commission rates $(M=27.63, S D=3.73$; corresponding $T$-score $M=66, S D=4)$ and atypically fast raw hit reaction times $(M=279.52, S D=25.53$; corresponding $T$-score $M=29, S D=7)$. 
To summarise CPT performance, group-level averages were inconsistent with a clinically impulsive profile. On average, hit reaction times were faster than average but not abnormally so, commission errors were not high, omission errors were relatively higher, and the Beta value supported a relatively even balance between speed and accuracy. Furthermore, the types of errors made during the task were more likely due to general attention lapses, particularly towards the end of the task. However, underlying normal group-level averages, approximately one quarter of the sample performed in a way that was consistent with clinically impaired motor impulse control; with atypically fast hit reaction times, a high number of commission errors and relatively fewer omission errors.

\subsubsection{Motor impulse control performance: Stop Signal Task}

As outlined in the Chapter 4, a sample of male university students $(N=26)$ was recruited to provide normative data for the Stop Signal task. Independent-sample $t$-tests were computed to compare offender and student performance on the Stop Signal task. All of the primary Stop Signal outcome scores from offenders and students are compared in Table $10^{13}$.

\footnotetext{
${ }^{13}$ Students were significantly younger than offenders. It is also likely students and offenders would differ by IQ. Meta-analytic studies show that IQ does not affect SSRT. Age-related SSRT differences are also more likely to affect young children and elderly adults, not young-to-middle adulthood groups (Lijffijt et al., 2005; van de Laar, van den Wildenberg, van Boxtel \& van der Molen, 2011). Thus, age and IQ were not treated as potential confounds in student-offender comparisons.
} 
Table 10. Offender and student raw score comparisons on the Stop Signal task

\begin{tabular}{|c|c|c|c|c|}
\hline $\begin{array}{c}\text { Stop Signal } \\
\text { outcome scores }\end{array}$ & $\begin{array}{l}\mathrm{M}(S D) \\
\text { Offender } \\
(N=65)\end{array}$ & $\begin{array}{l}\mathrm{M}(S D) \\
\text { Student } \\
(N=26)\end{array}$ & $t(89)$ & $D$ \\
\hline $\begin{array}{l}\text { Stop signal } \\
\text { reaction time } \\
(\mathrm{SSRT})\end{array}$ & $226.20(35.03)$ & $218.44(30.30)$ & 0.99 & 0.24 \\
\hline $\begin{array}{l}\% \text { missed } \\
\text { responses no- } \\
\text { signal trials }\end{array}$ & $3.90(5.21)$ & $1.52(4.55)$ & $2.03 *$ & 0.49 \\
\hline $\begin{array}{l}\text { Signal respond } \\
\text { reaction time }\end{array}$ & $714.75(153.55)$ & $542.10(167.54)$ & $4.72 * *$ & 1.07 \\
\hline $\begin{array}{l}\text { No-signal } \\
\text { reaction time }\end{array}$ & $804.15(152.61)$ & $571.54(234.03)$ & $5.59 * *$ & 1.18 \\
\hline $\begin{array}{c}\text { Stop Signal } \\
\text { Delay (SSD) }\end{array}$ & $577.59(157.75)$ & $383.57(185.87)$ & $5.03 * *$ & 1.13 \\
\hline
\end{tabular}

Note. All reaction time scores are measured in milliseconds, ${ }^{*} p<.05,{ }^{*} * p<.01$

As Table 10 shows, both groups differed in mean reaction time on trials with and without stop-signals: offenders took significantly longer to respond than students. Offenders were also able to successfully inhibit responses at significantly longer SSDs than students. Most importantly, there was no significant difference between mean SSRTs. Contrary to expectation, the time for the stopping process to reach completion did not take significantly longer for offenders than students. Both groups performed within the normal range with mean SSRTs between 200-250ms (F. Verbruggen, personal communication, May 19, 2009). 
The response patterns of each group suggest that offenders were taking a more strategic approach to the Stop Signal task than were students. Specifically, the combination of longer reaction times, longer SSDs, but equivalent mean SSRT in the offender group is consistent with the use of proactive adjustments made before each trial to allow successful inhibition at longer SSDs (Verbruggen \& Logan, 2009). Although participants are explicitly told at the beginning of the task not to wait for the stop signal because it compromises the speed of the 'go' reaction time, a number of studies show that healthy participants deliberately slow 'go' reaction times to ensure greater success of inhibition (e.g., Rieger \& Gauggel, 1999; Schachar et al., 2004; Verbruggen, Logan, Liefooghe \& Vandierendonck, 2008). The strategy of proactive adjustment suggests that offenders were carefully monitoring and adjusting their responses to favour accuracy over speed; a pattern we would not expect if motor impulse control were a significant problem in this group.

Perhaps the most substantial difference between the offender and student group was the necessity of data removal for 12 offender participants because their responses were too erratic to meet the threshold of successful inhibition approximately $50 \%$ of the time (Verbruggen, Logan \& Stevens, 2008). No such data removal was required for student data. The 12 offenders with erratic stop signal performance had comparable scores as offenders without erratic performance on the IGT (decision-making), CWIT and CTMT (perceptual impulse control). Similarly, commission and omission error rates on the CPT were comparable to offenders without erratic stop signal performance. However, offenders with erratic stop signal performance had significantly faster mean hit reaction times in the markedly atypical range $(M=275.76, S D=99.67)$, than offenders without 
erratic stop signal performance $(M=332.75, S D=51.42: t(68)=2.84, p<.01)$. As indicated by the CPT vigilance indicators, offenders with erratic stop signal performance also had lower $T$-scores (poorer performance) than offenders without erratic stop signal performance although the difference was non-significant. That is, offenders with erratic performance on the stop signal task provided atypically fast reaction times and had relatively more trouble sustaining attention throughout the task, than offenders without erratic performance on the stop signal task. This suggests that for a small proportion of offenders, response inhibition is likely to be impaired primarily due to general impairments in focusing and sustaining attention, rather than response inhibition impairment per se.

At a group level, the inhibition process took no longer to reach completion for offenders than it did for students: the SSRTs for both groups were within the normal range. To establish the proportion of participants with impaired performance embedded within the normal group-level indicators of performance, I used the student sample's performance parameters (SSRT $M=218, S D=30$ ) to create a threshold for impaired performance as SSRTs at least 2 SD longer than the student mean (i.e., $\geq 278 \mathrm{~ms}$ ).

Using this convention, only 5/65 (7.69\%) participants in the offender sample performed 2 SD outside the parameters of normative student performance, with an average SSRT of $293 \mathrm{~ms}(S D=8.42)$. Fourteen offenders $(21.54 \%)$ performed within 2 SDs of student performance (SSRT $M=259.37, S D=8.62)$, and 19/65 $(29.23 \%)$ performed within $1 \mathrm{SD}$ of student performance $(\mathrm{SSRT} M=232.24, S D=9.86)$.

In summary, only a small proportion - $7.69 \%$ —of the offender sample had a SSRT indicative of an abnormally slow inhibitory process. For the remainder of the 
sample, the speed of the inhibitory process was within the normal range. However, a subset of offenders had impaired performance from what appeared to be a generalised attention impairment causing erratic, variable responding. The CPT data for these offenders was consistent with the argument that response variability can be indicative of deficits in sustained attention (Leeth-Stensen, Elbaz \& Douglas, 2000). The combination of Stop Signal and CPT data for these offenders might also support the argument that response variability is itself an indicator of impulsiveness (Bellgrove, Hester \& Garavan, 2004).

\subsubsection{Proportions of impairment across domains}

A substantial proportion of performance was typically within the average, or below average range, but clinically impaired cognitive impulse control was not common. Strikingly, 28/77 (33.36\%) displayed no clinical impairment on any cognitive impulse control task. Participants without any deficits were no different from the remainder of the group with respect to age, IQ or static criminal risk. From a total of five tasks, 27/77 (35.06\%) displayed impairment on one task only, 21/77 (27.27\%) displayed impairments on two tasks, and only one participant displayed impairments on three tasks. Impairments in two or three tasks were not necessarily within the same domain of cognitive impulse control.

Recall that decision-making domain was only assessed using one task: as reported in section 5.3.3.1, 13\% of the sample displayed clinically impaired decision-making domain. Perceptual and motor impulse control domains each had two tasks. I counted the number of participants with impaired scores on both tasks used to measure perceptual and motor impulse control to establish impairment within a whole domain. 
For perceptual impulse control, only 3 participants, or $4 \%$ of the sample, was clinically impaired on both the CWIT and CTMT tasks in a way that impairment could not be explained by poor performance during baseline conditions. For motor impulse control, no participants were clinically impaired on both the CPT and Stop Signal tasks. However, it is important to note that these proportions relate exclusively to impulse control-specific deficits: there were indications of domain-general impairments in abilities such as sustained attention.

\subsection{General discussion}

The overarching objective of the present study was to assess whether cognitive impulse control was intact or impaired in a sample of repeat, high-risk adult offenders. Moffitt's (1993) LCP theory predicts that impairment in neuropsychological process like impulse control increases risk for lifelong involvement in antisocial behaviour.

Accordingly, my general prediction was one of pervasive impairment within and across all domains of cognitive impulse control.

\subsubsection{Empirical support for Bechara's framework}

In service of the first specific objective, it was necessary to test whether the sample's pattern of results across the 5 tasks were consistent with Bechara's threedomain framework. The hypothesis that outcome scores from the five independent tasks chosen to represent each domain would reduce to a three-component solution was supported. The outcome scores grouped together in a manner consistent with Bechara's three-domain framework; capturing decision-making, perceptual and motor impulse control domains. The pattern of results empirically supported the notion that there is unique variance in the skills captured within each domain and, assuming reliable 
assessment conditions are met, fractionation and isolation of weakness across domains is possible. Conceptually, the PCA results are consistent with previous studies highlighting the multidimensional nature of cognitive impulse control (e.g., Dougherty et al., 2009; Mathias, Marsh-Richard \& Dougherty, 2008), in addition to proposals that there are multiple pathways to impulsive behaviour (Gorlyn, Keilp, Tryon \& Mann, 2005).

\subsubsection{Evidence against clinically impaired cognitive impulse control overall}

Relatively less support was illustrated for the prediction of clinically impaired cognitive impulse control across all domains. Impairment tended to be isolated to specific tasks within each domain; particularly affecting cognitive flexibility. Although performance was not superior, typically falling within the average or slightly below average range, approximately one third of the sample displayed no impairment on any task, and not one offender was clinically impaired across all domains. Overall, the smaller than expected degree of cognitive impulse control impairment in the sample challenges Moffitt's prediction that stable, trait-level, neuropsychological deficits affecting the neural mechanisms that support impulse control maintain criminal risk into adulthood.

Overall, the findings suggest that by adulthood, cognitive impulse control functions could have matured, or were never impaired. However, it is important to emphasise that many aspects of cognitive functioning were far from superior; particularly regarding sustained attention, which appeared to increase response variability on motor impulse control tasks. Generalised cognitive deficits of this kind support Moffitt's prediction of sup-optimal neuropsychological functioning for LCP offenders, but they do not support an impulse control-specific deficit per se. 


\subsubsection{Specificity of impairment: exploring the unique complexities of each task}

The largest proportions of clinical impairment were found for the inhibition/switching condition on the CWIT, and for CPT performance. Although the CWIT represented the perceptual impulse control domain and the CPT represented the motor impulse control domain, the PCA results suggested that each task draws on similar functions: arguably, cognitive flexibility. Impairments in cognitive flexibility are consistent with findings from a number of previous offender studies (e.g., Bergvall, Wessely, Forsmen \& Hansen, 2001; Broomhall, 2005; Hancock, Tapscott \& Hoaken, 2010). Interestingly, the smallest proportion of impaired performance (7.8\%) was observed on the Stop Signal Task; the complementary motor impulse control task. Because the outcomes from the CPT and Stop Signal task loaded on the same latent factor, the difference in impairment rates on each task was surprising. If the tasks were tapping the same latent construct, it follows that impairment on one task should equate to impairment on the other task. One explanation for this disparity could be that the removal of Stop Signal data for some offenders led to underestimations of impairment on both motor impulse control tasks. A further possibility is that whilst both tasks capture the essence of motoric inhibition, the CPT captures additional cognitive skills—including cognitive flexibility — accounting for relatively more impaired performance on the CPT (Miyake \& Friedman, 2012).

Previous work suggests that the Stop Signal task is a relatively purer measure of motoric inhibition than tasks like the CPT, which tax both response selection and response inhibition processes (Rubia et al., 2001). In the Stop Signal task, the 'go' response is required $75 \%$ of the time; thus, the 'go' response becomes dominant and 
participants only have to focus on stopping. On the other hand, the CPT requires dual skills of successfully deciding whether to provide a 'go' or 'stop' response (i.e., is the letter an $\mathrm{X}$ or non-X?), as well as the ability to successfully implement the stopping process. Arguably, quickly considering the appropriate response option, and inhibiting distraction from stimuli that closely match the non-target (e.g., the letter Y), will tax cognitive flexibility. Consistent with the notion that the CPT required both response selection and inhibition processes, inhibition/switching completion times on the CWIT cross-loaded on the motor impulse control factor. The fact that impairment was most pronounced on both CWIT inhibition/switching and on the CPT suggests that regardless of domain — whether impulse control is required at the level of thoughts or actual behaviour-cognitive inflexibility was the most impaired ability.

More generally, indications of general impairment in sustained attention might also account for the difference in impairment rates on the CPT and Stop Signal tasks. The ability to orient, divide and sustain attention generally facilitates response inhibition (Coull, 1998; Diamond, 2013). Although speculative, the CPT could have taxed attention relatively more than the Stop Signal task, reducing the availability of cognitive resources needed to maintain successful response inhibition. The CPT runs continuously for 14 minutes and provides no feedback about performance. On the other hand, the Stop Signal task separates each test block of 64 trials with a 10 second break, and provides on-screen feedback on performance accuracy. Whilst a short break has the obvious advantage of respite from mental fatigue, behavioural observations made during the testing session revealed that performance feedback could have served a motivational function. Many participants expressed a desire to better their performance in each round after receiving 
feedback on their speed and accuracy in the previous round. Perhaps the provision of feedback in the Stop Signal task boosted motivation — and possibly physiological arousal — to an optimum level, which consequently kept attention engaged (Revelle, 1987). In contrast, the continuous monotony of the CPT task and the absence of motivational cues might have contributed to waning vigilance; and consequently, less successful response inhibition. Previous studies support the influence of motivational context on response inhibition (see Leotti \& Wager, 2009).

The differences between the cognitive demands of each motor impulse control task are supported by neuroimaging studies showing that dorsolateral prefrontal regions are activated during response selection, whilst orbitofrontal prefrontal regions are activated during response inhibition (e.g., Horn et al., 2003; Rubia et al., 2001). The possibility that each tasks taps different aspects of motor impulse control is also supported by Dougherty and colleagues (2009) who found that scores from one variant of the Continuous Performance Test and a variant of a the Stop Signal task loaded on different factors. Similarly, Dolan and Fullam (2002) illustrated dissociable performance on the Stop Signal task and a Go/No go task (similar to the CPT), in a sample of personality-disordered offenders.

Overall, the pattern of impairment in this study was more closely aligned with explanations of impulsivity as failures in perceptual impulse control—specifically, cognitive flexibility—-than with reward-sensitivity explanations. A similar impairment differential has been observed for probationers diagnosed with Antisocial Personality Disorder (Swann, Liffjit, Lane, Kjome, Steinberg \& Moeller, 2009), and for adults with a history of Axis I and II disorders (Swann, Bjork, Moeller \& Dougherty, 2002). 
Moffitt's (1993) LCP theory makes predictions about the etiological role of inhibitory deficits in maintaining criminal risk, but the theory does not make specific predictions about the possible role of increased reward-sensitivity. Thus, even though the low level of reward sensitivity observed in this study cannot be directly compared to predictions of Moffitt's LCP theory, comparisons can be made to other theoretical predictions, such as theories of psychopathy.

Psychopathy is a prevalent personality disorder in New Zealand's high-risk offenders who attend the STURPs (Polaschek, 2009). Passive-avoidance theories of psychopathy suggest that psychopaths are impervious to punishment; thus, their behaviour is often marked by dysfunctional impulsivity (Patrick, Fowles \& Krueger, 2009). Despite the likelihood that the majority of offenders in this sample meet the criteria for psychopathy ${ }^{14}$, only $13 \%$ of the sample exhibited decision-making indicative of reward-sensitivity. For the remainder of the sample, choice preferences were particularly oriented to the avoidance of frequent punishment. Therefore, the data was inconsistent with passive-avoidance theories of psychopathy.

Even for the small proportion of offenders whose decision-making was on par with neurological patients and consistent with hypersensitivity to reward, clinical impairment in the ability to develop and use somatic markers to guide decision-making is not the only explanation for their impaired performance. Previous studies show that some neurologically healthy people perform disadvantageously by choosing more cards from bad decks: typically, this disadvantageous performance pattern is accompanied by

\footnotetext{
${ }^{14}$ The VRS and VRS-SO include an item - criminal personality - that assesses characteristics of psychopathic personality (Wong \& Gordon, 2000). Ratings on this item indicated that criminal personality was a treatment need for 40/56 (71\%) participants with VRS ratings, and for 12/15 (80\%) participants with VRS-SO ratings.
} 
normal, but relatively weaker physiological responses during anticipation of choice from bad decks, compared to neurologically healthy people who perform advantageously (Bechara, Damasio, Damasio \& Lee, 1999). Bechara and colleagues (1999) found that approximately $20 \%$ of neurologically healthy adults who performed disadvantageously described themselves as risk-takers. Thus, it is argued that high risk-takers will override weak physiological responses that would ordinarily signal avoidance of risky options, by higher cognitive processes (Bechara, Damasio \& Damasio, 2000).

Given that a number of higher cognitive processes were intact in the present sample, deliberate override of avoidance, and approach of riskier decks, is one alternative explanation for the small proportion of clinically impaired decision-making performance. Crucially, deliberate override of physiological responses signaling avoidance of risky options is a different type of impairment to the inability to generate physiological responses during the decision-making process (Bechara, Damasio \& Damasio, 2000; Miller, 1992). Following inspection of the block net scores for the 10 participants who displayed clinically impaired decision-making, only 3 participants had impaired block net scores throughout the whole task. On the other hand, 7 participants displayed the ability to learn; with block net scores that gradually increased from blocks 1-3, then decreased in blocks 4-5. Rather than impairments in generating the affective information that assists the decision-making process, this pattern is more closely aligned with deliberate choice not to apply those affective signals to choices; best conceptualised as risk-taking or selective impulsivity whereby impulses are resisted only when impulse control is judged to be important (Broomhall, 2005). 


\subsection{Summary}

Overall, cognitive impulse control was not clinically impaired for the majority of high-risk, adult offenders suggested to represent Moffitt's LCP type. When performance was impaired, it was isolated to tasks that taxed cognitive flexibility. It is unclear whether offenders in this sample never had impaired cognitive impulse control ability, or whether they had reached a stage in adulthood in which the neural mechanisms that facilitate better impulse control were more fully developed. The possibility of maturation is of theoretical interest given that developmental theories of antisocial behaviour, like Moffitt's (1993) LCP theory, assert that stability in cognitive impulse control impairment contributes to the maintenance of criminal risk across the life course. The following chapter explores the notion of stability and the possibility of change in cognitive impulse control following a cognitive-behavioural correctional rehabilitation programme. 


\section{Chapter 6}

\section{Changes in cognitive impulse control following treatment}

\subsection{Introduction}

Developmental theories of antisocial behaviour, like Moffitt's (1993) developmental taxonomy and Gottfredson and Hirschi's (1990) self-control theory suggest that stable impulse control impairments maintain criminal risk. However, empirical evidence challenges the stability assumption by showing that there are unique, fluid developmental patterns of impulse control over the life span (e.g., Hay \& Forrest, 2006; Higgins, Jennings, Tewksbury \& Gibson, 2009; Ray, Jones, Loughran \& Jennings, 2013). Moreover, some research suggests that executive functions - including those related to impulse control-are malleable in adulthood in response to training and practice (e.g., Brehmer, Westerberg, \& Backman, 2012; Jolles, van Buchem, Rombouts \& Crone, 2012; Karbach \& Kray, 2009; Riggs, Greenberg, Kusche, \& Pentz, 2006; Rothwell, La Vigna \& Willis, 1999; Salminen, Strobach \& Schubert, 2012; Wiers, Gladwin, Hofmann, Salemink \& Ridderinkhof, 2013). A helpful analogy is provided in the self-regulation literature, whereby impulse control is described as a muscle: the more the 'muscle' is used, the stronger regulatory abilities become across different behavioural domains (Baumeister, 2002; Muraven, Baumeister \& Tice, 1999). In this chapter, I argue that attendance of a cognitive-behavioural rehabilitation programme that requires, models, promotes and positively reinforces regulated behaviour could provide the opportunity for cognitive impulse control to be consistently practiced and subsequently strengthened. 


\subsection{The treatment context}

New Zealand's Special Treatment Unit Rehabilitation Programmes (STURPs) deliver treatment based on a cognitive-behavioural therapy (CBT) framework. The treatment programme itself, and the prison unit overall, are based on principles of social learning theory (Polaschek \& Kilgour, 2013). As outlined in Chapter 4, the STURPs are set within a therapeutic community of change. The ethos of a therapeutic community denotes that, in addition to the treatment material taught in the group room, one of the primary mechanisms of positive change is engaging in functional interactions with other treatment participants, custodial and therapeutic staff (Polaschek \& Kilgour, 2013). The custodial staff members are informed about treatment plans for individual treatment participants; thus, both custodial and therapeutic staff monitor change behaviour closely.

Treatment participants are expected to uphold their role as a responsible community member, whether they are in the group treatment room or spending leisure time in the compound (Van Rensburg, Berry, Rutherford, Williams, Whitehead \& van Rensburg, 2009). Any behaviour that undermines the community or treatment process is grounds for premature departure from the treatment programme. Attendance of weekly community meetings provide the opportunity for treatment participants to individually or collectively take ownership for any behaviour that has undermined the treatment process, or to acknowledge and praise each other's positive behaviour (Polaschek \& Kilgour, 2013). Unlike mainstream prison units where offenders' time is often less structured and behaviour is primarily monitored for security reasons only, the STURPs provide a highly structured environment in which time is spent constructively, and treatment participants know their behaviour is constantly monitored for evidence of change behaviour. 
Within the CBT framework, specific treatment modules address topics that aim to assist offenders in becoming better regulators of their impulses, including: distress tolerance; mindfulness; self-control: mood and anger management; and safety planning for release. Attitudes and behaviours that impede successful impulse control are challenged, with the aim of helping offenders to develop new behavioural management strategies. However, unlike other types of cognitive rehabilitation, treatment is not designed to target neurocognitive impairments that might be underlying impulsive behaviour (Cicerone, 2002; Cicerone \& Giacino, 2002). Nevertheless, the combination of CBT-based treatment sessions and the therapeutic community overall denotes that the treatment environment requires, promotes and positively reinforces practice of regulated behaviour (Denney, 1984). Accordingly, it is possible that offenders who attend STURPs could develop better cognitive impulse control skills as a by-product of immersion in such a structured environment with clear rewards for regulated behaviour. Although it remains a source of debate in the literature, previous research suggests that the neural plasticity of the adult brain denotes that improvements in cognitive impulse control abilities are possible in response to practice and training.

\subsection{Plasticity of the adult brain: training-related changes to neural structures and cognitive functioning}

The plasticity of the adult nervous system allows for change in neural structures in response to training and practice (Draganski et al., 2004; Gage, 2002). For example, Holzel and colleagues (2011) reported increased gray matter concentration in subjects who took part in an 8-week, mindfulness-based stress reduction course. Specifically, post-training increases in gray matter concentration — thought to occur from repeated 
activation of particular brain regions - were observed in neural regions responsible for learning and memory, emotion regulation, self-referential processing, and perspectivetaking (Holzel et al., 2011, May et al., 2007).

In addition to structural changes, other findings support the plasticity and trainability of cognitive functions. Again, mindfulness training has been implicated in improvements in voluntary, top-down attentional skills (Jha, Krompinger \& Baime, 2007). Outcomes from training interventions for patients with Traumatic Brain Injury (TBI), also show that skill re-training via behaviour translates to performance improvements at a neurocognitive level (see Cicerone, Levin, Malec, Stuss \& Whyte, 2006 for a comprehensive review). Von Cramon, Matthes-Von Cramon and Mai (1991) recruited patients with TBI and other cerebral insults who displayed poor problem solving on controlled tests of planning and response regulation. Approximately half of the participants received remediation training in problem orientation, problem definition and formulation, generation of alternatives, decision-making and solution verification. The remainder of the sample received, as an alternative, a memory-retraining intervention. Participants in the memory-retraining group exhibited minor improvements, whilst participants in the remediation intervention showed significant improvements on planning ability (as measured by the Tower of Hanoi puzzle), and on behavioural ratings of everyday problem-solving behaviour.

Relatedly, Rath and colleagues (2003) delivered an innovative or conventional intervention of equal intensity ( 24 sessions), to outpatients with TBI. The innovative intervention focused on motivational, attitudinal and affective processes and problemsolving skills (i.e., problem orientation, problem solving, using self-regulation strategies 
to stop emotional over reactions). The conventional intervention focused on high-level applications of five basic skills: awareness of strengths and deficits, attention, notetaking, giving and getting feedback, and social skills. A psychosocial component was also included whereby participants discussed coping with emotional reactions and behavioural changes post-injury. Participants were compared on measures of executive function (attention, memory, reasoning), problem solving and objective observer-rated assessments of behaviour during naturalistic role-play scenarios. Following the intervention, both groups displayed improved memory functioning but only the innovative group displayed significant improvements on measures of executive functioning, self-appraisal of emotional regulation and clear thinking, and objectively rated interpersonal problem solving during role-plays. Moreover, the positive gains made in the innovative group were maintained 6 months following treatment.

Other interventions for TBI providing generalised, as opposed to task-specific, training of regulatory cognitive processes have led to improved functioning in patients with working memory deficits (Cicerone, 2002), impairments in controlling inappropriate responses (Alderman, Fry \& Youngson, 1995), perseverative problems (Cicerone \& Giacino, 1992), and impairments in self-regulation and self-awareness (Ownsworth, McFarland \& Young, 2000). However, in spite of some promising results, other researchers have highlighted important limitations of cognitive training paradigms. To date, the most substantial limitation concerns inconsistent support for the generalisation of task-specific training-related improvements to untrained tasks and to everyday behaviour outside of the laboratory (e.g., Logie, 2012; Shipstead, Kenny \& Engle, 2012). 


\subsection{The treatment context: more opportunity to practice impulse control, or more opportunity to fail?}

Even when interventions are specifically directed toward improving neurocognitive skills, the efficacy of such interventions is still under debate. Although the STURPs are not designed to address neurocognitive functioning, the treatment framework shares similar characteristics to the training components of effective TBI and mindfulness interventions that encourage and facilitate practice of controlled behaviour. Therefore, there are reasons to expect that the nature of the treatment context could provide treatment participants with important opportunities for practicing —and potentially strengthening-impulse control.

Whether offenders enter treatment from an intrinsic desire to change, or from a desire to impress the parole board to achieve early release, the ultimate goal is still treatment completion: completing an 8-month, intensive treatment programme presents many challenges and tests of impulse control. Intact decision-making skills are required for developing long-term goals like treatment completion and planning for life after release. During treatment sessions, perceptual and motor impulse control skills will be taxed when inhibition of unhelpful responses is required or when flexible consideration of new goals is necessary. The group dynamic also requires that offenders must simultaneously process treatment material and manage their social environments, requiring resistance to cognitive interference (Fishbein et al., 2009).

Accordingly, another important possibility to consider is that the unique challenges of the treatment context could tax cognitive impulse control skills beyond capacity for some offenders. For example, offenders with poor cognitive flexibility skills 
may find it difficult to simultaneously navigate their social environment and process treatment material effectively. Essentially, sub-optimal cognitive impulse control is a double-edged sword: it represents a potential criminogenic need requiring treatment intervention, as well as a specific responsivity issue that could hinder an offender's chances of making treatment progress (Andrews \& Bonta, 2006; Ward, Day, Howells \& Birgden, 2004). For that reason, it is reasonable to expect that offenders who exhibit the poorest pre-treatment cognitive impulse control would be more likely to prematurely leave the treatment programme for responsivity or conduct-related problems.

Published research linking pre-treatment neurocognitive ability to treatment outcomes in the context of cognitive-behavioural therapy with incarcerated adult offenders appears to be limited to one study conducted by Fishbein and colleagues in 2009. Neurocognitive functioning was assessed before treatment and treatment outcomes were evaluated after the first phase of the rehabilitation programme. After controlling for other factors known to contribute to treatment attrition (e.g., IQ, age, education), Fishbein and colleagues found that poorer pre-treatment behavioural inhibition and inability to shift responses based on new information differentiated offenders who left, versus completed, the first treatment phase. In addition to completion of the initial treatment phase, they also found that better behavioural inhibition was associated with greater treatment responsivity and gain. These results highlight the possible influence of cognitive impulse control on the capacity to engage in, and complete treatment. 


\subsection{Study objectives and hypotheses}

It is possible that cognitive impulse control could improve in an environment that requires, promotes, and positively reinforces controlled behaviour. The first objective of this study is to investigate whether cognitive impulse control improves, declines or stays the same following treatment. It is hypothesised that cognitive impulse control improvements will be observed given the unique requirements of the treatment context, which calls for intensive practice of impulse control. However, no a priori predictions are made regarding the magnitude of improvement, or whether improvements will be similar, or different, across cognitive impulse control domains (decision-making, perceptual and motor impulse control).

It is also possible that the intensity and social complexity of the treatment context might tax cognitive impulse control ability beyond capacity for some offenders, leading to poorly controlled behaviour that undermines the treatment process, and subsequent premature departure from the treatment programme. To contribute to literature on responsivity factors and possible markers of treatment attrition, the second objective of this study is to determine whether treatment completers and non-completers are differentiated on the basis of pre-treatment cognitive impulse control. Based on previous literature, I hypothesised that treatment non-completers would have relatively poorer pretreatment cognitive impulse control than treatment completers; no a priori predictions are made regarding the specificity or generality of poorer performance in relation to different cognitive impulse control domains. 


\subsection{Method}

\subsubsection{Statistical analyses}

The treatment change data presented here is from all offenders who participated in the pre-treatment research assessment, completed the treatment programme and consented to the post-treatment assessment (see Chapter 4 for a full description of the pre-treatment sample). For some cases, data from treatment completers had been removed during pre-treatment analysis for various reasons (see Chapter 4). Thus, only participants with valid pre and post-treatment data for each task were included in the present analysis (see Appendix 8 for a summary).

Pre-to-post treatment changes were assessed using paired $t$-tests. Comparisons between treatment completers and non-completers were assessed using independentsamples $t$-tests. To aid in the interpretation of results, Cohen's $d$ (the effect size index for repeated measures; Cohen, 1988) was calculated. The effect size calculation was based on the average standard deviation from two means and correction for dependence between two means (Morris \& DeShon, 2002). According to Cohen, effect size conventions for $d$ are: small $=.30$, medium $=.50$, large $=.80$.

\subsubsection{Participants}

Of the 77 participants who participated in the pre-treatment research assessment, $58(75.3 \%)^{15}$ completed the treatment programme, spending an average of $256(S D=19)$ days in the core treatment programme. Of the 19 treatment non-completers, premature departures from treatment were due to: assault on a custodial staff member $(n=1)$, poor

\footnotetext{
${ }^{15}$ This completion rate is similar to rates identified in other jurisdictions (e.g., Losel, 2001; Nunes \& Cartoni, 2006) and consistent with evaluation studies of STURP participants between 19982004 and between 2003-2011 (Polaschek, 2010; Polaschek \& Kilgour, 2013).
} 
responsivity $(n=2)$, unspecified misconduct $(n=13)$ and voluntary withdrawal for personal reasons $(n=3)$. The average number of days spent in treatment before departure was highly variable for treatment non-completers: some offenders never started the core group and others spent substantial time in treatment before departure $(M=114, S D=$ 107). Of the 58 treatment completers, 50 participants took part in the post-treatment research assessment (3 participants declined participation and 5 participants could not be reached for the research assessment before transfer to other prisons). Treatment completers and non-completers did not differ significantly on age, IQ, or static criminal risk.

\subsubsection{Procedure}

The procedure for post-treatment data collection was identical to pre-treatment data collection (see Chapter 4; see also Appendices 3 and 4 for copies of post-treatment information and consent forms). Participants were administered the cognitive impulse control battery in the same order of task presentation they received pre-treatment. At the end of the session, participants were debriefed about the research objectives. Participants were also given the opportunity to leave an address of a family member in the community so that results of the research could be posted out once the study was completed. The mean number of days between pre and post-treatment assessments was $280(S D=32)$.

\subsection{Results}

The primary outcome scores for each task were compared pre and post-treatment. The discussion of change scores below is again focused primarily on outcome scores with central relevance to Bechara's operationalisation of decision-making, perceptual and motor impulse control. 


\subsubsection{Decision-making performance: Iowa Gambling Task (IGT; $N=50)$}

Recall that total net scores reflect the total number of card selections from good decks minus bad decks $(\mathrm{CD}-\mathrm{AB})$. A positive total net score reflects advantageous performance and a negative net score reflects disadvantageous performance. Posttreatment total net scores $(M=18.8, S D=32.6)$ were significantly higher than pretreatment total net scores $(M=7.4, S D=24.1: t(49)=2.7, p<.01, d=.38$. Significantly more advantageous decision-making following treatment was further illustrated by a total net $T$-score increase from below the normative mean of 50 pre-treatment $(M=48, S D=$ 7), to above the normative mean post-treatment $(M=52, S D=12)$.

Pre-treatment, although participants obtained positive total net scores overall, they preferred Decks B and D—low punishment frequency decks—not Decks C and D as per Bechara's (2007) predictions for optimal, advantageous decision-making. I wanted to establish whether the same deck preferences held, or changed post-treatment. Accordingly, I conducted a 4 (deck) x 5 (block) within-subjects repeated measures ANOVA on post-treatment gambling task data. For the sake of comparison, pre-treatment (Figure 1) and post-treatment (Figure 2) deck selections by block are presented together and discussed below. 


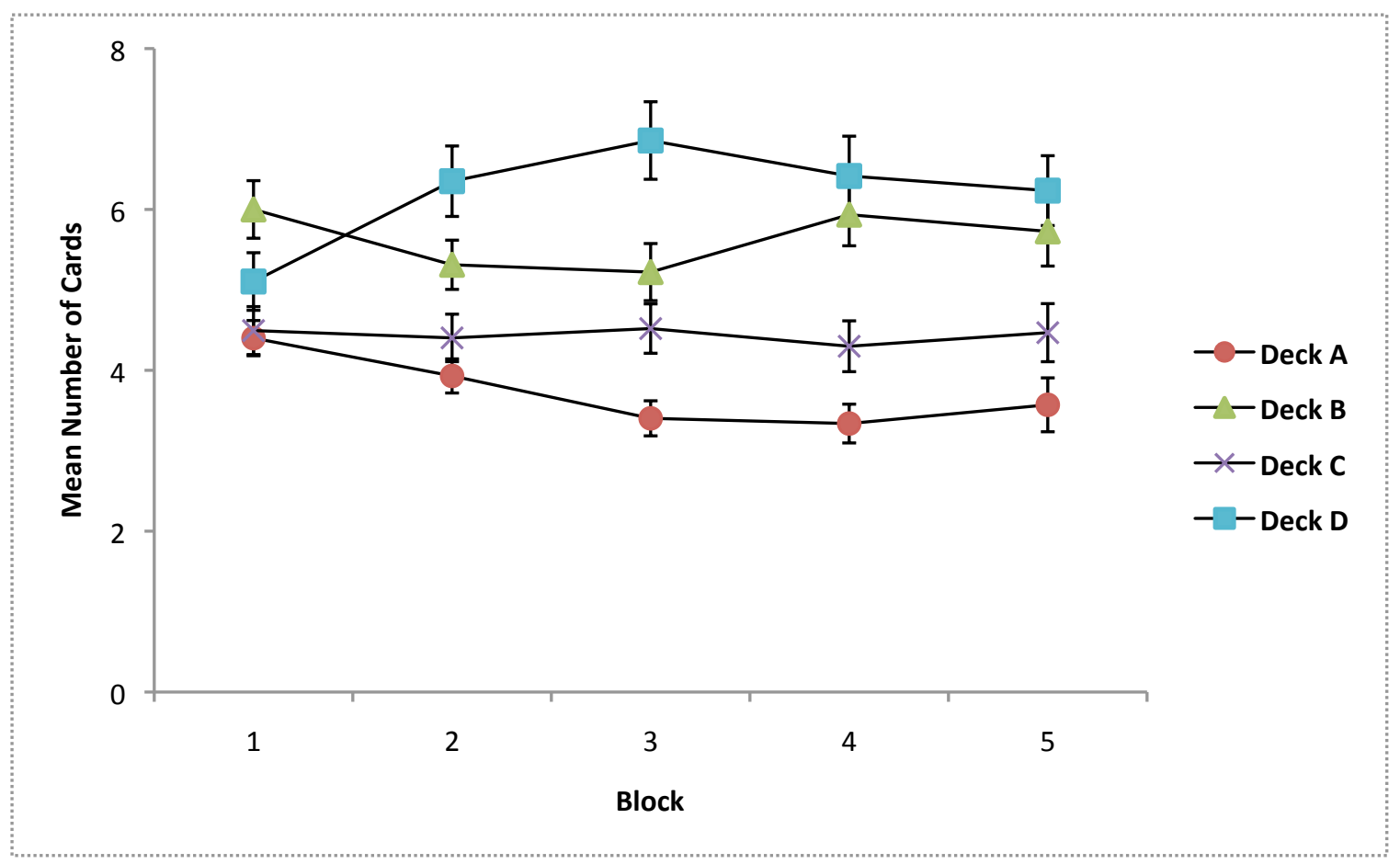

Figure 1. Pre-treatment mean number of cards selected from each deck over five blocks of 20 choices. Bars represent $+/-1 \mathrm{SE}$ of the mean.

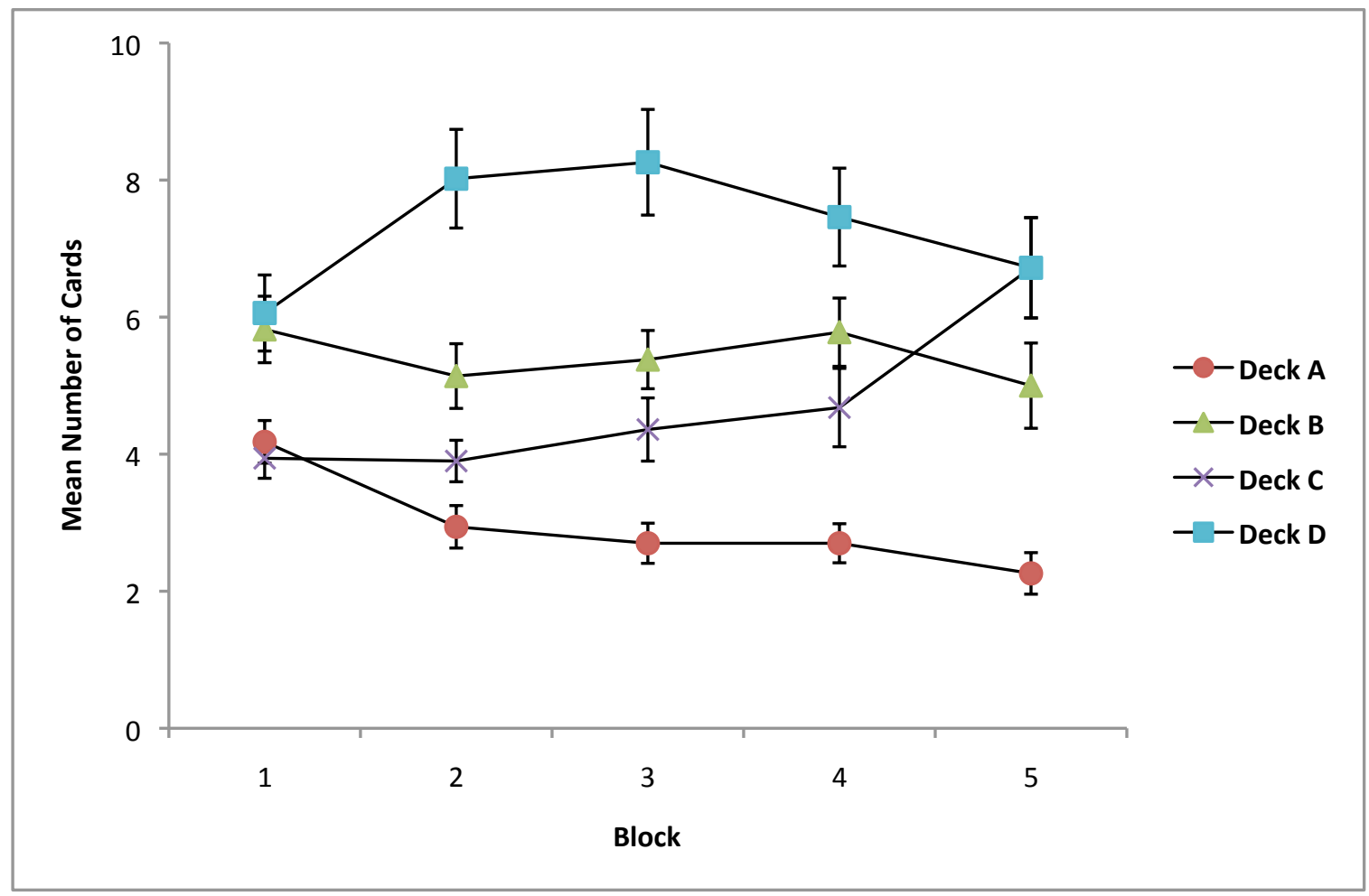

Figure 2. Post-treatment mean number of cards selected from each deck over five blocks of 20 choices. Bars represent $+/-1$ SE of the mean. 
There was a significant main effect for card deck: $F(3,47)=34.95, p<.001$. That is, irrespective of block, there were significant differences in the number of card selections from each deck. Identical to pre-treatment deck selections, the number of selections from Deck A $(M=2.6)$ was significantly fewer than the number of selections from Deck B $(M=5.2)$, Deck C $(M=4.6)$, and Deck D $(M=7.3$; all comparisons, $p<$ .001). Pre-treatment, the number of selections from Decks B and D were very similar; however, post-treatment, significantly more selections were made from Deck D than Deck B $(p<.05)$. Deck D was also selected significantly more often than Deck C $(p<$ $.001)$.

There was a significant Block x Deck Interaction: $F(3,47)=3.41, p=<.01$. In other words, the number of card selections made from each deck differed on the basis of which stage participants were in the task. To investigate the trends in deck selections across all 5 blocks in the task, four follow-up repeated measures ANOVAs were conducted for each deck.

\subsubsection{Deck A (Large reward, large frequent losses, low net gain)}

There was a significant effect of block on card selections from Deck A: $F(4,46)$ $=8.65, p<.001$. The overall pattern of selections over blocks best fitted a linear function: $F(1,49)=24.40, p<.001$. Pairwise comparisons showed that card selections from Deck A decreased in a negative linear fashion from earlier to later blocks. Specifically at Block 1, the mean number of card selections from Deck A was significantly higher than selections at Block $2(p<.01)$, Block $3(p<.01)$, Block $4(p<.01)$ and Block $5(p<$ .001). Compared to pre-treatment performance, post-treatment data illustrate more marked avoidance of Deck A indicated by lower mean selections overall. 


\subsubsection{Deck B (Large reward, large infrequent losses, low net gain)}

There was no significant effect of block on card selections from Deck B: $F(4,46)$ $=0.90, p=.47$. As is evident when comparing Deck B in Figure 1 (pre-treatment) and Figure 2 (post-treatment), the mean number of selections from Deck B per block was at, or below, the pre-treatment mean selections from this deck. The decreased tendency to sample from Deck B post-treatment might illustrate participants' recognition that decks with higher gain outcomes $(\mathrm{C}$ and $\mathrm{D})$ were relatively more favourable than a deck with low punishment frequencies, but high punishment magnitude (Deck B).

\subsubsection{Deck C (Small reward, small frequent losses, high net gain)}

There was no significant effect of block on card selections from Deck C: $F(4,46)$ $=2.06, p=.10$. Although selections gradually increased from earlier to later blocks to reach their maximum in the final block, the increases were not statistically significant. However, the upward linear trend in Deck C selections post-treatment (Figure 2), is still important to consider given that the pattern is in direct contrast to pre-treatment Deck C selection patterns (Figure 1). Pre-treatment, selections fitted a flat trend that did not change across blocks and Deck $\mathrm{C}$ was avoided almost as much as Deck A: the most disadvantageous deck in terms of net gain. Yet, as Figure 2 illustrates following treatment, in the final block of selections, the preference for Deck C surpasses a previous favourite—Deck B — and almost reaches a similar level of selections as Deck D. Whereas pre-treatment choices did not reflect recognition of the overall net gain value of Deck C, the post-treatment pattern shows that participants gradually learned the value of Deck C throughout the game and eventually came to favour overall outcome (i.e., net gain), over 
and above low loss frequency which was favoured most pre-treatment, as evidenced by the highest selections from Deck B and D.

\subsubsection{Deck D (Small reward, small infrequent losses, high net gain)}

There was no significant effect of block on the number of cards selected from Deck D: $F(4,46)=1.92, p=.12$. Deck D was uniformly preferred the most across all blocks. The post-treatment mean total selections from Deck D $(M=35.9, S D=14)$ were significantly higher than pre-treatment $(M=30.6, S D=12.5: t(49)=2.8, p<.01)$. This suggests that participants recognised the dual benefits — best net gain outcome and low loss frequency_ of Deck D even more following treatment.

\subsubsection{IGT decision-making performance summary}

Overall, post-treatment decision-making was relatively more advantageous than pre-treatment decision-making, moving closer to Bechara's operationalisation of optimal decision-making as a preference for choices that secure the best long-term outcomes. Two consistent patterns emerged that were relatively more pronounced following treatment: avoidance of Deck A (bad deck) and preference for Deck D (good deck). Consistent avoidance of the high-risk Deck A indicates that participants were not lured by high reward. Instead, participants recognised the high-risk nature of Deck A and quickly learned to avoid high-risk choices. The consistent preference for Deck D shows that participants' choices were ultimately guided by low loss frequency and overall outcome (i.e., best net gain).

The selection patterns for Decks B and C changed (though not always significantly) from pre-to-post treatment. Deck B returns high reward, and high but infrequent punishment. Pre-treatment, Deck B was preferred nearly as much as Deck D. 
However, after treatment, a greater number of selections were made from Deck D indicating that participants chose more according to long-term outcome (Deck D), and were less likely to take the gamble of infrequent but severe punishment (Deck B). This pattern is consistent with the pre-to-post treatment change in selections from Deck C. Deck C was avoided nearly as much as Deck A before treatment. However, after treatment, towards the final blocks of the task, selections from Deck C increased nearly paralleling selections from Deck D. That is, a shift occurred from a preference for low loss frequency before treatment to low loss magnitude — and therefore better long-term outcome - after treatment. Taking a snapshot of performance in the final block of selections post-treatment, performance was consistent with Bechara's notion of optimal decision-making: favouring of Decks $\mathrm{C}$ and $\mathrm{D}$ relative to Decks A and B. In other words, choice patterns were more risk-averse following treatment.

\subsubsection{Perceptual Impulse Control: Colour Word Interference Test (CWIT; N=49)}

Performance interpretation on the Colour-Word Interference test (CWIT) is based on raw completion time (seconds), error rates, scaled scores and contrast scaled scores. Scaled scores allow comparisons with normative data $(M=10, S D=3)$. Longer completion times represent more difficulty in each condition, whilst higher scaled scores represent better performance.

Relative to pre-treatment scores, indicators of performance improvements include: faster raw completion times with a similar or lower level of errors and a higher scaled score. Indicators of performance decline include: longer completion times, a higher level of errors and a lower scaled score. Table 11 displays pre-post treatment comparisons of raw completion times and raw total errors (corrected and uncorrected) for each condition. 
Table 11. Pre- to post-treatment comparison of raw completion time (seconds) and errors on the CWIT

\begin{tabular}{lcccccccc}
\hline & \multicolumn{3}{c}{ Pre } & \multicolumn{2}{c}{ Post } & & & \\
\cline { 2 - 6 } CWIT outcome scores & $\mathrm{M}$ & $S D$ & $\mathrm{M}$ & $S D$ & $d f$ & $t$ & $d$ \\
\hline Colour naming & 29.6 & 5.5 & 29.1 & 5.4 & 48 & 1.4 & .19 \\
Word reading & 22.7 & 4.3 & 23.2 & 4.4 & 48 & 1.4 & .20 \\
Inhibition & 56.4 & 12.3 & 53.7 & 13.6 & 48 & $2.4^{*}$ & .36 \\
Inhibition/switching & 73.9 & 22.2 & 71.1 & 17.4 & 48 & 1.5 & .22 \\
Inhibition Errors & 3.1 & 2.8 & 2.8 & 3.2 & 48 & 0.7 & .11 \\
$\begin{array}{l}\text { Inhibition/switching } \\
\text { Errors }\end{array}$ & 3.9 & 3.8 & 3.4 & 3.1 & 48 & 1.3 & .19 \\
\hline Note. ${ }^{*} p<.05$ & & & & & & &
\end{tabular}

\subsubsection{CWIT raw completion time and error summary}

As Table 11 shows, with the exception of the word reading condition, completion times were faster post-treatment, though only significantly so for the inhibition condition. The inhibition and inhibition/switching conditions are most relevant to understanding changes in perceptual impulse control. Inhibition completion time decreased significantly with a small to medium effect size following treatment. Together with the finding that inhibition error rates decreased — though not significantly—-the results suggest a small improvement, whereby participants were faster at inhibiting a habitual response following treatment which was unlikely to be explained by a speed-accuracy trade-off. The small decrease in completion time for the inhibition condition denoted that post-treatment scaled scores $(M=9.9, S D=2.9)$, were significantly higher than pre-treatment scaled scores $(M=9.0, S D=3.1)$, shifting performance closer to the normative mean of 10 : $t(48)=3.1, p<.01, d=.30$. 
Inhibition/switching completion times were faster following treatment, although the difference did not reach statistical significance. Error rates were also lower in this condition post-treatment, again, non-significantly. Once again, the results represent a small post-treatment shift towards better cognitive flexibility and inhibition, all with fewer errors than before treatment. However, despite the small post-treatment improvement, pre-treatment inhibition/switching scaled scores $(M=7.2, S D=3.7)$ and post-treatment inhibition/switching scaled scores $(M=7.6, S D=3.5)$ remained nearly 1 $\mathrm{SD}$ below the normative mean of 10 . This suggests that even following treatment, participants had sub-optimal — though not clinically impaired — cognitive flexibility.

\subsubsection{Perceptual Impulse Control: Trail Making Test (CTMT; N=49)}

Pre and post-treatment comparisons were calculated for the Composite Index (CI) and raw completion times for each trail: all were converted to $T$-scores $(M=50, S D=10)$ for normative comparison. Lower raw completion times and higher $T$-scores indicate better performance. Mean post-treatment CI $T$-scores $(M=51.1, S D=10.3)$ were significantly higher than pre-treatment $(M=46.9, S D=10.5)$, indicating significantly better post-treatment trail-making performance overall, which shifted closer to the normative mean of 50: $t(48)=3.8, p<.001, d=.55$. Table 12 displays the pre-post treatment raw completion time comparisons for each individual trail. 
Table 12. Pre- to post-treatment comparison of raw completion times on the CTMT

\begin{tabular}{cccccccc}
\hline \multicolumn{7}{c}{ Pre } & \multicolumn{7}{c}{ Post } \\
\hline Trail & $M$ & $S D$ & $M$ & $S D$ & $d f$ & $t$ & $d$ \\
\hline Trail 1 & 35.1 & 11.9 & 31.2 & 10.1 & 48 & $2.9 * *$ & 0.42 \\
Trail 2 & 31.8 & 10.4 & 28.1 & 9.3 & 48 & $2.9 * *$ & 0.42 \\
Trail 3 & 36.6 & 13.8 & 33.8 & 12.4 & 48 & 1.5 & 0.22 \\
Trail 4 & 34.5 & 15.3 & 32.1 & 12.8 & 48 & 1.1 & 0.17 \\
Trail 5 & 59.5 & 24.8 & 52.4 & 18.9 & 48 & $3.1 * *$ & 0.47 \\
\hline Note. $* * p<.01$ & & & & & & &
\end{tabular}

As shown in Table 12, participants took less time to complete all five trails

following treatment; though the differences were not always significant. Completion time per trail includes time taken for self-corrected or examiner-corrected errors; any errors made in sequencing must be corrected before participants move on. Therefore, reduced post-treatment completion time could not be explained by a change in speed-accuracy trade-off.

Trails 4 and 5 are most relevant for understanding changes in perceptual impulse control, specifically cognitive flexibility or set shifting ability. As displayed in Table 12, Trail 4 completion times did not improve significantly following treatment. Accordingly, mean pre-treatment $T$-scores $(M=46.4, S D=13.1)$ were comparable to mean posttreatment $T$-scores $(M=47.9, S D=11.5)$. Trail 5 completion times improved significantly following treatment. This translated to significantly higher mean posttreatment $T$-scores $(M=46.8, S D=10.3)$, compared to pre-treatment $(M=43.3, S D=$ 
10.4): $t(48)=3.2, p<.01, d=.46$. Taken together, despite a statistically significant improvement for completion time on Trail 5 , set-shifting ability on both trails remained below the normative mean following treatment, consistent with set-shifting performance on the CWIT.

\subsubsection{Motor Impulse Control: Continuous Performance Test (CPT; $N=45)$}

The CPT manual states that the specific combination of atypically fast hit reaction time, a high number of commission errors and relatively fewer omission errors represent an impulsive performance profile (Conners, 2002). Mean hit reaction time increased significantly from pre-treatment $(M=330.5, S D=52.7)$ to post-treatment $(M=346.9, S D$ $=73.9): t(44)=2.4, p<.05, d=.39$. Raw mean commission errors decreased from pretreatment $(M=18.1, S D=8.8)$, to post-treatment $(M=16.8, S D=8.8)$, though the difference was not significant. Raw mean omission errors increased from pre-treatment $(M=5.0, S D=5.3)$ to post-treatment $(M=6.4, S D=8.5)$, but again, the difference was not significant.

Considering the increase in hit reaction time, the small decrease in commission errors and a small increase in omission errors, the results are consistent with a slightly more cautious, less impulsive approach to responding post-treatment. A relatively less impulsive profile following treatment was further supported by $T$-score changes $(M=50$, $S D=10$ ). For hit reaction time, low $T$-scores (unusually fast reaction times) indicate impulsivity (Conners, 2002). The mean pre-treatment hit reaction time $T$-score was 39 $(S D=11)$, and the mean post-treatment hit reaction time was $42(S D=14)$. Although low, neither pre nor post-treatment hit reaction time mean $T$-scores were atypically low (i.e., atypically fast). However, it is still promising that the post-treatment hit reaction 
time distribution shifted from the impulsive end of performance closer towards the normative mean: whereas pre-treatment hit reaction time was more than 1 SD below the normative mean, post-treatment reaction time was within 1 SD below the normative mean.

For commission errors, higher $T$-scores represent higher error rates. The mean pre-treatment commission error $T$-score was $54(S D=10)$ and post-treatment was 53 ( $S D$ $=10$ ). Therefore, participants performed slightly worse (though within $1 \mathrm{SD}$ ), than the normative sample both before and after treatment, responding to non-targets when inhibition was required.

\subsubsection{Motor Impulse Control: Stop Signal $(N=39)$}

Recall that the Stop Signal task estimates the speed of the inhibition process via the Stop Signal Reaction Time (SSRT). The normal range for SSRT is between 200250ms (personal communication, Verbruggen, 19 May, 2009). Mean pre-treatment SSRTs $(M=226.42, S D=32.19)$, and mean post-treatment SSRTs $(M=226.34, S D=$ 44.23), were almost identical; and at both time points, the speed of participants' inhibitory process was within the normal range.

However, it is important to note that data from 11 participants had to be removed from pre-post treatment comparisons because responses were erratic and the required $50 \%$ response inhibition threshold for estimating SSRT was not reached (Verbruggen, Logan \& Stevens, 2008). Performance for these 11 participants on the other cognitive impulse control tasks was mostly comparable to performance by participants with reliable Stop Signal data. However, on the CPT - the complementary motor impulse control task - participants with erratic stop signal performance had poorer vigilance scores, 
indicative of difficulty with sustained attention. As in the pre-treatment analyses outlined in Chapter 5, again, for a small sub-set of offenders, response inhibition likely remained impaired following treatment due to a more generalised deficit in remaining oriented to the task over time, rather than because of an inhibitory deficit per se.

\subsubsection{General summary: patterns of change}

Participants who completed treatment and agreed to the post-treatment assessment, showed small pre-to-post treatment improvements on some cognitive impulse control outcomes, but mostly improvements were not significant. The improvements were found in all three domains of cognitive impulse control, but they were task-dependent (i.e., limited to one task only) for domains that were assessed using two different tasks (perceptual and motor impulse control).

Regarding decision-making on the gambling task, comparisons against normative data suggested that treatment completers' decision-making improved from below the normative mean pre-treatment, to above the mean following treatment. This was likely due to the shift in pre-treatment deck preference for decks with the lowest frequency punishment before treatment, to decks with the best long-term outcome post-treatment. Although preference for low punishment frequency decks is consistent with the choice patterns of neurologically healthy, non-offender participants (e.g., Lin, Song, Chen, Lee \& Chiu, 2013; Steingrover, Wetzels, Hortsmann, Neumann \& Wagenmakers, 2013), it was not the optimal decision-making strategy as defined by Bechara (Bechara, 2007). Thus, the shift from over valuing avoidance of punishment pre-treatment, to approaching the best long-term reward post-treatment, reflects a shift toward Bechara's operationalisation of optimal decision-making. 
In the perceptual impulse control domain, basic inhibition significantly improved on the Colour-word Interference Test. In other words, by the end of treatment, participants displayed significantly better ability to resist cognitive interference generated by habitual, but incorrect impulses. However, treatment change was less consistent for the cognitive flexibility aspect of perceptual impulse control. Even if significant improvements were observed, post-treatment cognitive flexibility performance was still approximately $1 \mathrm{SD}$ below normative means. Thus, cognitive flexibility remained the most impaired function following treatment.

In the motor impulse domain, small improvements were observed on the Continuous Performance Test. Specifically, post-treatment performance reflected a slightly more cautious approach to responding; reactions were slowed, and thus, responding was slightly more accurate. However, the time taken to inhibit a response on the Stop Signal task was no different following treatment. Evaluation of response patterns on both motor impulse control tasks indicated that even after treatment, a small sub-set of offenders still found it difficult orienting to, and remaining focused on the tasks. Thus, improving motor impulse control functions over the course of treatment could have been impeded by weaknesses in the fundamental cognitive mechanisms that support impulse control.

\subsubsection{Comparisons of pre-treatment cognitive impulse control between treatment} completers and non-completers

Raw scores for each cognitive impulse control task were compared between offenders who went on to complete treatment $(N=58)$ and those who did not $(N=19)$. Sample sizes for treatment completer and non-completer groups vary for different reasons 
(see Table 13). Higher raw scores for IGT total net (decision-making) and hit reaction time (CPT) represent better, less impulsive performance. Lower raw scores for inhibition and inhibition/switching (CWIT), Trail 4 and 5 (CTMT) and commission errors (CPT) represent better, less impulsive performance.

Table 13. Comparison of pre-treatment cognitive impulse control between treatment completers and non-completers (raw scores)

\begin{tabular}{|c|c|c|c|c|c|c|c|c|c|}
\hline \multirow[t]{2}{*}{ Outcome score } & \multicolumn{3}{|c|}{ Completer } & \multicolumn{3}{|c|}{ Non-completer } & \multirow[b]{2}{*}{$d f$} & \multirow[b]{2}{*}{$t$} & \multirow[b]{2}{*}{$d$} \\
\hline & M & $S D$ & $N$ & M & $S D$ & $N$ & & & \\
\hline IGT Total net & 6.45 & 24.29 & 58 & 4.21 & 26.77 & 19 & 75 & .34 & .09 \\
\hline Inhibition & 55.91 & 11.89 & $56^{\mathrm{ab}}$ & 57.22 & 9.49 & $18^{\mathrm{b}}$ & 72 & .43 & .12 \\
\hline Inhibit/switching & 72.89 & 18.63 & $56^{\mathrm{ab}}$ & 79.22 & 16.96 & $18^{\mathrm{b}}$ & 72 & 1.3 & .36 \\
\hline Trail 4 & 33.76 & 14.44 & 58 & 32.95 & 9.89 & 19 & 75 & .23 & .07 \\
\hline Trail 5 & 59.59 & 21.59 & 58 & 63.00 & 19.48 & 19 & 75 & .61 & .17 \\
\hline Hit reaction time & 326.03 & 49.29 & $56^{\mathrm{cd}}$ & 321.67 & 41.63 & $18^{\mathrm{c}}$ & 72 & .34 & .09 \\
\hline $\begin{array}{l}\text { Commission } \\
\text { errors }\end{array}$ & 18.18 & 8.00 & $56^{\mathrm{cd}}$ & 18.94 & 7.82 & $18^{\mathrm{c}}$ & 72 & .35 & .09 \\
\hline SSRT & 224.29 & 32.82 & $51^{\mathrm{e}}$ & 233.18 & 42.82 & $14^{\mathrm{e}}$ & 63 & .84 & .23 \\
\hline \multicolumn{10}{|c|}{$\begin{array}{l}\text { Note. }{ }^{\mathrm{a}} \text { Outlier removal, }{ }^{\mathrm{b}} \text { Refusal to complete task, }{ }^{\mathrm{c}} \text { Did not complete task due to } \\
\text { unavoidable prison interruptions }{ }^{\mathrm{d}} \text { Did not complete task due to computer malfunction }{ }^{\mathrm{e}} \\
\text { Data removal: erratic responding }\end{array}$} \\
\hline \multicolumn{10}{|c|}{ Contrary to expectation, Table 13 shows that there were no significant pre- } \\
\hline
\end{tabular}




\subsection{General discussion}

\subsubsection{Small, selective cognitive impulse control changes}

The first objective of this study was to investigate whether cognitive impulse control improved, declined or remained the same following treatment. The findings partly supported the hypothesis that offenders who completed treatment would show improvements in cognitive impulse control abilities. But for the most part, improvements were small and non-significant.

Decision-making on the Iowa Gambling Task improved significantly, attributable to a shift following treatment towards favouring long-term outcomes over and above immediate avoidance of punishment. Regarding perceptual impulse control abilities, completion times for basic inhibition on the Colour-word Interference Test also reflected better ability to resolve cognitive interference and successfully inhibit an over learned, habitual response following treatment. However, performance changes on conditions taxing inhibition and cognitive flexibility were less consistent: even if scores significantly improved, they generally remained below normative means. Essentially, cognitive flexibility remained the most sub-optimal cognitive impulse control ability following treatment.

In the motor impulse control domain, hit reaction times were significantly longer and commission errors decreased slightly, indicating a small shift towards less impulsive performance following treatment. There were no significant changes regarding time to inhibit a response on the Stop Signal task. However, Stop Signal performance again showed that, in addition to sub-optimal cognitive flexibility, a small sub-set of offenders 
had persisting difficulties with general cognitive abilities like attention, even after treatment.

\subsubsection{Explanations for small post-treatment changes}

Given that the treatment programme was not specifically intended to improve neurocognitive functioning, it is important to consider why improvement occurred on some tasks; and also, why change did not occur on others. One explanation to consider is that post-treatment improvement simply reflected regression to the mean. However, because pre-treatment scores were not typically extremely low or high, regression to the mean may be an insufficient explanation. Another possible explanation to consider is the influence of practice effects (McCaffrey, Ortega \& Haase, 1993). Explicit practice effects refer to memory for specific test items, learned strategies for solving problems, or general experience and comfort with testing (McCaffrey, Duff \& Westervelt, 2000).

For the majority of tasks included in this study's assessment battery, practice effects typically occur when re-test intervals are much shorter than the average re-test interval of 280 days in this research. On the Trail-making test for example, Buck, Atkinson and Ryan (2008) found that the task was sensitive to practice effects as evidenced by decreased trail completion times across three test administrations. However, the delay between each testing session was only 7 days. I argue that a substantially longer average delay of 280 days without exposure to the tasks between assessments in the present study would decrease the likelihood of explicit practice effects (McCaffrey, Ortega \& Haase, 1993). Still, in a relatively recent meta-analysis, Calamia and colleagues (2012) showed that re-test intervals of 2-3 years are needed on tests of verbal comprehension and knowledge, verbal memory, visual memory, reasoning, and working 
memory to completely eliminate score gains. It is unclear whether such substantial intervals are necessary for the cognitive impulse control tasks I used in this study.

One reason to expect that the performance improvements were not exclusively attributable to explicit practice effects is that significant performance improvements were not observed for all outcomes. If all of the tasks were susceptible to practice effects over an approximately 9-month-long delay, we might expect improvement to have affected more outcomes, and possibly to a larger degree. For example, on the Iowa Gambling Task, it took participants until the final block (last 20 of 100 card selections) to begin performing in accordance with optimal decision-making, as per Bechara's (2007) operationalisation of preference for Decks C and D. If participants remembered which decks returned the most money from their pre-treatment assessment, presumably they would have started making advantageous selections much earlier in the task to maximise advantageous performance.

To fully understand mechanisms of change, research of this kind would benefit greatly from: 1) better access to test-retest reliability data for all tasks, and 2) the inclusion of a matched, control group of offenders who undergo assessments separated by the same amount of time as treated offenders, but without attending the treatment intervention. At present, it remains a limitation of this study that I was unable to disentangle how or why treatment completers made small post-treatment improvements on some cognitive impulse control tasks. Investigating these questions remains an important avenue for future research. Nevertheless, the fact that some small improvements were observed at all has important implications for developmental theories of antisocial behaviour. 


\subsubsection{Theoretical and clinical implications of changes (or lack of)}

Although based on speculation at this point, the small improvements could have occurred as a by-product of the treatment context which provided: consistent practice with engaging attention and other general cognitive abilities during treatment sessions, adhering to rules, engaging in positive interactions with others, and being accountable for behaviour. Practicing behaviours of this kind could have indirectly boosted impulse control-specific functions, or more general cognitive functions, which subsequently facilitated small impulse control improvements by the end of treatment (e.g., Riggs, Greenberg, Kusche, \& Pentz, 2006; Rothwell, La Vigna \& Willis, 1999; Salminen, Strobach \& Schubert, 2012; Wiers, Gladwin, Hofmann, Salemink \& Ridderinkhof, 2013).

The possibility of training cognitive abilities to function more effectively via practice is consistent with theories of self-regulation (e.g., Baumeister, 2002; Baumeister \& Heatherton, 1996; Baumeister, Heatherton \& Tice, 1994). The notion that cognitive impulse control functions might be amenable to change is also consistent with previous work suggesting that developmental trajectories of impulse control are fluid, not fixed (e.g., Hay \& Forrest, 2006; Higgins, Jennings, Tewksbury \& Gibson, 2009; Ray, Jones, Loughran \& Jennings, 2013). The possibility of practice-related improvements is at odds with developmental theories of antisocial behaviour, which imply that inhibitory deficits function like a stable trait to maintain criminal risk (e.g., Hirschi \& Gottfredson, 1990; Moffitt, 1993). Although the change findings are very preliminary and require replication, they highlight the possibility that conceptualising cognitive impulse control as a fixed, trait-like offender characteristic may not be empirically supported. Instead, 
these abilities may be better conceptualised as representing an individual difference with the potential for further development in environments that support, promote and positively reinforce their practice. This possibility is discussed in further detail in Chapter 8 (General Discussion).

The fact that some functions did not improve is also important to consider. One possible explanation is that skills within different cognitive impulse control domains develop at different rates: cognitive flexibility, for example, develops later than more basic inhibitory skills (Davidson, Amso, Anderson \& Diamon, 2006; Garon, Bryson \& Smith, 2008). Accordingly, perhaps functions that naturally come later in development are also functions that would require more intense, direct intervention for development in adulthood (Jolles \& Crone, 2012). Whilst basic inhibition improved significantly following treatment, for the most part, cognitive flexibility did not. Perhaps daily practice of selectively attending to information delivered in the treatment sessions whilst blocking out other environmental distractions boosted general ability to resist cognitive interference. On the other hand, cognitive flexibility may represent a relatively more complex function with unique requirements that could not be sufficiently trained by the general treatment context alone (Miyake \& Friedman, 2012).

Together, small patterns of change suggest that the treatment context may go some way in facilitating better impulse control. However, the mechanisms of this change are unknown and represent an important avenue for future research. The treatment intervention was never intended to target neurocognitive functions; however, the absence of post-treatment change on some outcomes and the maintenance of sub-optimal functioning in some areas speak to the possibility that more direct training interventions 
may be required for developing higher-level, complex cognitive abilities required for impulse control.

\subsubsection{No differentiation between treatment completers and non-completers}

The second objective of this study was to investigate whether treatment completers and non-completers had different patterns of pre-treatment cognitive impulse control performance. The hypothesis that treatment completers would display relatively better pre-treatment cognitive impulse control performance than treatment noncompleters was not supported. Completers and non-completers could not be differentiated on age, IQ, static criminal risk, or pre-treatment cognitive impulse control.

This finding is in contrast to Fishbein and colleagues' (2009) study, which showed that treatment non-completers performed significantly worse than treatment completers on pre-treatment tests of inhibition and set shifting. However, the similarities between treatment completers and non-completers is generally consistent with other work showing that treatment non-completion is influenced by a range of factors, that may be unrelated to specific offender characteristics (e.g., McMurran \& McCulloch, 2007). Indeed, previous comparisons of treatment completers and non-completers from New Zealand STURPs reveal very few differences on demographic, criminal history and psychometric indices that have been found to predict treatment non-completion in other research (e.g., Polaschek, 2010a; Polaschek \& Kilgour, 2013).

\subsubsection{Cognitive impulse control as a responsivity factor?}

Although pre-treatment cognitive impulse control did not account for variation in treatment completion, the question remains as to whether the pattern of some small but significant improvements in specific cognitive impulse control functions following 
treatment can explain variation in other variables of theoretical and practical interest. That is, perhaps individual variation in cognitive impulse control is best conceptualised as a responsivity variable that influences treatment response, rather than treatment completion per se. The indications that some participants still had inflexible cognition and poor sustained attention following treatment raises important questions around how well such offenders were able to learn about novel treatment-related concepts. Moreover, if the learning process was impaired, the capacity to implement new skills developed in treatment could also have been substantially limited.

Because impulse control is necessary for adaptive functioning across a number of domains, arguably, variation in cognitive impulse control might explain change—or lack of - in other areas of criminal risk. Given the resource-intensive nature of effective cognitive-rehabilitation programmes for high-risk offenders, it is particularly important to establish whether cognitive impulse control might facilitate change in other areas of functioning. The following chapter explores this issue with specific reference to posttreatment change in criminal risk and emotion regulation. 


\section{Chapter 7}

\section{Cognitive impulse control, criminal risk, and anger}

\subsection{Introduction}

To fully understand the clinical significance of cognitive mechanisms of impulse control and to develop theoretical frameworks to guide clinical formulation and treatment interventions, it is important to investigate how cognitive impulse control is related to

criminal risk overall, and to other risk factors. Ultimately, frameworks of this kind would not only facilitate greater understanding of impulse control, but also contribute to currently limited theory regarding how improvements on one criminal risk factor can engender change in other risk factors (Marshall, 1997; Polaschek \& Kilgour, 2013; Polaschek, 2010b; Ward, Polaschek \& Beech, 2005). Impulse control is crucial for adaptive behaviour in many ways; therefore it is likely that impulse control would have important influences on functioning in a range of other areas of criminal risk. One area of risk that impulse control may be particularly important for is in the regulation of emotional experience and expression; both impulse control and emotion regulation are treatment targets for reducing criminal risk for violent and non-violent offending (Polaschek \& Collie, 2004).

This study explores the relationships between cognitive impulse control, criminal risk and anger-related outcomes. Although there are many risk factors that cognitive impulse control ability is likely to affect, anger-related outcomes - specifically trait anger and anger control—were selected in this study for theoretical reasons. Fortunately, the 
STURP psychometric databases contained data on anger-related variables that could be related to the cognitive impulse control measures collected here.

\subsection{How might cognitive impulse control be related to criminal risk, trait anger and anger control?}

Moffitt's developmental taxonomy predicts that variation in cognitive impulse control—inhibitory deficits—explains variation in criminal risk (Moffitt, 1993).

Theoretically then, better impulse control should predict lower criminal risk. However, empirical tests of this prediction are lacking in adult offender populations (Farrington, 2011). Moffitt's LCP theory also predicts that anger is one component of the disinhibited, difficult temperament typically observed in offenders on the LCP path (Colder \& Stice, 1998; Moffitt, 1993). Findings from genetic studies are consistent with Moffitt's assertion: anger and impulse control share genetic variance and thus, angry people also tend to have poor impulse control (Gagne \& Goldsmith, 2011). However, whilst Moffitt's theory speaks to the etiological relevance of co-occurring inhibitory deficits and negative emotionality for individuals on the LCP path, the theory does not specify how each component might influence the other to contribute to difficult temperament, and consequent antisocial risk. There are three possible ways that cognitive impulse control and anger outcomes may be causally related; the directions of causal relationships are likely to have different implications for clinical formulation and treatment intervention.

According to Spielberger (1999), trait anger refers to a person's general disposition to become angry across a variety of situations, particularly when they feel unfairly treated or criticised. Anger control refers to the effort expended to control verbal and physical expressions of anger (Spielberger, 1999). Given the depleting effect of 
emotion on cognitive resources, one causal direction could be that higher trait anger depletes the cognitive resources needed for anger control, thus causing frequent failure of anger control (Anderson \& Bushman, 2002; Baumeister \& Heatherton, 1996; Denson, Pederson, Friese, Hahm \& Roberts, 2011). Put simply, dysfunctional emotional experience comes first: high trait anger interferes with cognitive functions, which reduces the capacity for already weak inhibitory abilities to exert control over the expression of emotion.

A second causal relationship could be in the direction of sub-optimal cognitive impulse control limiting ability to influence emotions; specifically, the intensity of the emotions generated, and if or how they are expressed (Gross, 2002; Ochsner \& Gross, 2004). Previous studies support the notion that cognitive processes serve to shape emotions and therefore, sub-optimal functioning of cognitive impulse control mechanisms leads to dysfunctional emotional experience and poor emotion regulation (MacLeod \& Bucks, 2011; Mathews \& MacLeod, 2005; Whitmer \& Banich, 2010; Williams, Watts, MacLeod \& Mathews, 1997). From this view, sub-optimal cognitive functioning comes first: dysfunctional cognitive processing interferes with normal emotional experience and regulatory ability.

Emotion regulation theorists suggest that cognitive mechanisms can exert their influence at early or late stages of the emotional regulation process; initially affecting the intensity of the emotion generated, and later, whether that emotion is expressed or inhibited (e.g., Gross \& Thompson, 2007). At the stage of emotion generation, cognitive impulse control functions are pivotal in the use of cognitive strategies that modulate the intensity of emotion. For example, the ability to use effortful mental strategies that rely 
on cognitive impulse control facilitates the reduction of subjective and physiological experience of anger (Denson, 2013; Rusting \& Nolen-Hoeksema, 1998). Two examples of such strategies include cognitive reappraisal—consciously reinterpreting the meaning of a situation to alter the emotional response to it (e.g., Hooker \& Knight, 2006) - and distraction. Reappraisal allows adaptive processing of anger-related information, whilst distraction allows for rapid reduction in the subjective experience of anger (Denson, Moulds \& Grisham, 2012). Distraction requires attentional focus on non-emotional thoughts or stimuli, and the inhibition/suppression of those that are the triggers of anger (Denson, 2013).

The use of cognitive reappraisal strategies have been shown to reduce sympathetic nervous system activation following recall of an anger-invoking event, highlighting the important role of cognitive strategies in reducing physiological symptoms of anger (Ray, Wilhelm \& Gross, 2008). Neuroimaging studies also show co-activation of neural regions of the prefrontal cortex during cognitive-emotional control (e.g., Bunge, Ochsner, Desmond, Glover \& Gabrieli, 2001; Ochsner, Ray, Cooper, Robertson, Chopra, Gabrieli \& Gross, 2004; Pessoa, 2005, 2009; Pessoa et al., 2002; see also Denson, 2013 for a review). Collectively, findings to date illustrate the important relationships between cognitive and emotional processes in emotion regulation. However, cognitive processing might not necessarily precede emotional processing or vice versa; rather, cognitive and emotional processes could work synergistically in a back and forth manner to influence emotion regulation.

Therefore, the third causal relationship could be reciprocal: if people with high trait anger ruminate because they cannot distract themselves from angry feelings, rumination 
will maintain or increase both angry feelings and physiological symptoms of anger (Bushman, Bonacci, Pedersen, Vasquez \& Miller, 2005; Glyn, Christenfeld \& Gerin, 2002). Consequently, high trait anger will be maintained and the cycle of cognitive resource depletion and failure to modulate the intensity of emotion and control of emotion will be perpetuated (Quigley \& Tedeschi, 1996).

In summary, there are theoretical reasons to expect that cognitive impulse control will be causally related to: 1) criminal risk, 2) trait anger 3) anger control, but the direction(s) of the causal relationships is yet to be established. Establishing the directionality of the relationships has important theoretical implications for understanding the interplay between impulse control and other risk factors. Moreover, establishing directionality of causal relationships has important clinical implications: if variation in cognitive impulse control predicts other risk-related outcomes, cognitive impulse control abilities could influence how much treatment-related change offenders can make in other important areas of criminal risk.

\subsection{Study objectives and hypotheses}

To date, empirical investigations of Moffitt's LCP prediction that poorer cognitive impulse control contributes to greater criminal risk are lacking. Additionally, a theoretical understanding of how cognitive impulse control and emotion (and particularly anger) regulation are related remains a great source of debate in psychological literature generally, but theory is especially underdeveloped in the forensic literature. Developing theoretical frameworks for understanding: 1) whether there are causal relationships between cognitive impulse control, overall criminal risk, and anger outcomes, and 2) the 
direction of those causal relationships, is crucial for theory, clinical formulation and correctional rehabilitation interventions.

Specifically, if high trait anger predicts poorer cognitive impulse control, developing strategies to reduce the frequency and intensity of angry feelings would represent a primary treatment target that could also assist the development of better impulse control, and possibly anger control. On the other hand, if sub-optimal cognitive impulse control predicts higher trait anger and poorer anger control, treatment intervention strategies to develop impulse control may have cascading effects for modulating trait anger and anger control. If the relationships are reciprocal, interventions that develop cognitive impulse control and emotion-specific interventions are likely to have a beneficial effect on each other.

Using a pre-post treatment design, the overarching objectives of this study were to establish empirical support for predictions based on Moffitt's (1993) LCP theory in a sample of adult — presumably LCP — offenders. Moffitt's LCP theory proposed that deficits in impulse control maintain criminal risk. Therefore, it was predicted that poorer pre-treatment cognitive impulse control would be associated with higher pre-treatment criminal risk. Assuming that offenders reduce criminal risk following the treatment intervention, it was also hypothesised that better pre-treatment cognitive impulse control would predict lower post-treatment criminal risk.

Previous research supports the possibility that any of the three causal relationships between cognitive impulse control, trait anger and anger control outlined earlier may be observed. However, given the large body of research supporting the facilitative role of cognitive mechanisms of impulse control in emotion regulation, I specifically tested the 
hypothesis that cognitive impulse control would influence the modulation of emotional experience and regulation. Accordingly, it was hypothesised that poorer pre-treatment cognitive impulse control would be associated with higher pre-treatment trait anger and poorer pre-treatment anger control. Assuming that offenders make anger-related improvements following treatment, it was also hypothesised that better pre-treatment cognitive impulse control would predict lower post-treatment trait anger and better posttreatment anger control.

\subsection{Method}

\subsubsection{Participants}

Of the 77 offenders assessed pre-treatment, 58 completed treatment. Fifty participants consented to the post-treatment research assessment: 3 participants declined participation, and 5 participants could not be reached for the research assessment in time before transfer to other prisons (see Appendix 8 for a summary of valid pre-post treatment data for each cognitive impulse control task).

\subsubsection{Materials}

Consistent with materials used previously throughout this thesis (see Chapter 4 for a full description of each task), decision-making was assessed with the IOWA Gambling Task (IGT), perceptual impulse control was assessed with the Colour-Word Interference Test (CWIT) and the Comprehensive Trail Making Test (CTMT) and motor impulse control was assessed with the Conners' Continuous Performance Test (CPT) and the Stop Signal Task. Criminal risk was assessed using the Violence Risk Scale (VRS), which reliably estimates risk of violent and non-violent offending and was therefore used as an estimate of general criminal risk (Wong \& Gordon, 2000; 2006). As outlined in Chapter 
4, the VRS is a clinician-rated risk assessment tool based on 6 static risk items and 20 dynamic risk items that are theoretically and empirically linked to violence. Static risk items are based on criminal history variables (e.g., age at first violent conviction) that remain unchanged regardless of treatment intervention. In contrast, dynamic risk items capture changeable characteristics relating to an offender's general lifestyle, attitudes, behaviour, personality and social network. Because dynamic risk factors are amenable to change, they represent potential treatment targets (Wong \& Gordon, 2000).

\subsubsection{State-Trait Anger Expression Inventory (STAXI-2; Spielberger, 1999).}

Anger-related variables were assessed using the most recent version of Spielberger's State-Trait Anger Expression Inventory (STAXI-2). The STAXI-2 includes 57 self-report items rated on a 4 -point Likert scale $(1=$ almost never, $4=$ almost always $)$. The STAXI-2 has three sub-scales: state anger, trait anger, and anger expression and control. The state anger sub-scale (15 items) captures "in-the-moment" angry feelings: how much the respondent feels like expressing angry feelings verbally or physically at the time of assessment. The trait anger subscale (10 items) captures angry temperamenta general tendency to become angry — and angry reaction, or the tendency to respond with anger particularly in response to feeling ill-treated or criticised by others. The anger expression and control subscales ( 32 items) capture the extent to which anger is expressed or controlled, both inwardly (i.e., suppression of anger) and outwardly (i.e., aggressive actions toward others).

Raw STAXI-2 data are summed and converted to percentiles and $T$-scores for normative comparison. For the purpose of the present study, raw trait anger and anger control (outward) subscale scores were included in the analysis based on their theoretical 
relevance to impulse control. Trait anger scores represent how often angry feelings are experienced over time and context. Anger control (outward) scores represent how much energy individuals expend to monitor and control explosive manifestations of anger (Spielberger, 1999). That is, high trait anger scores reflect more anger, whilst high anger control scores reflect better anger control. The STAXI-2 is a revision and expansion of the original STAXI. Internal consistencies for the STAXI-2 across scales and subscales were adequate to excellent $(\alpha=73-.94)$ across gender and community and psychiatric samples (McCloskey \& Coccaro, 2003). Data on test-retest reliability is not provided in the STAXI-2 manual; however, the STAXI has adequate test-retest reliability $(\alpha=.62$ .81; McCloskey \& Coccaro, 2003). The STAXI-2 manual provides correlations of trait anger scores with other personality scales in support of convergent validity (STAXI-2 Manual; Spielberger, 1999, Chapter 5).

\subsubsection{Procedure}

The procedure for the administration of cognitive tasks for the research assessment was outlined in the General Method (Chapter 4). The STAXI-2 questionnaires were administered independently from the research assessment as part of routine, pre and post-treatment psychometric testing conducted by therapists in the STURPs (Polaschek \& Kilgour, 2013). The exact length of time between pre and post-treatment psychometric assessment could not be calculated because STAXI-2 assessment dates were unavailable. The mean length of time between pre and post-treatment research assessments was 280 days (9.2 months, $S D=32$ days, range 251-398 days). Given that both psychometric and research assessments were conducted in the pre and post-treatment assessment phases of 
the treatment programme, it is likely STAXI-2 assessments were separated by a similar time lag to the research assessments.

\subsubsection{Data analysis}

Seven of the 50 offenders in the pre-post treatment sample were incarcerated for a sexual offence. Accordingly, their pre and post-programme criminal risk assessments were conducted using the Violence Risk Scale-Sexual Offender Version (VRS-SO; Olver, Wong, Nicholaichuk \& Gordon, 2007). Because VRS-SO scores are not easily converted to VRS scores, data for 43 participants were available for VRS analyses. Similarly, 2 of the 50 offenders were not administered the STAXI at all, as part of the routine psychometric assessment and 6 participants only had pre-treatment data, leaving data for 42 participants in the STAXI analyses.

First, descriptive statistics and paired-sample $t$-tests were computed to assess whether criminal risk, trait anger, and anger control changed following the treatment intervention. Establishing if, and in what direction, change in criminal risk and anger variables occurs is the first necessary step in testing the hypothesis of whether pretreatment cognitive impulse control predicts change in criminal risk and anger variables.

Second, Pearson product-moment correlations were computed between the pretreatment outcomes from each of the 5 cognitive impulse control tasks, VRS dynamic criminal risk, STAXI trait anger and STAXI anger control. Third, a series of cross-lag correlations were computed using AMOS (Version 19) to establish the directionality of relationships between cognitive impulse control and: 1) VRS dynamic criminal risk 2) STAXI trait anger, 3) STAXI anger control. Because all variables were assessed pre and post-treatment, this provides the basic foundation for cross-lag correlations between two 
constructs measured at two points in time. Two constructs measured at two time points generate four variables $\left(\mathrm{X}_{1}, \mathrm{X}_{2}, \mathrm{Y}_{1}, \mathrm{Y}_{2}\right)$ and six correlations: 2 autocorrelations $\left(\mathrm{X}_{1} \mathrm{X}_{2}\right.$; $\left.\mathrm{Y}_{1} \mathrm{Y}_{2}\right), 2$ synchronous correlations $\left(\mathrm{X}_{1} \mathrm{Y}_{1} ; \mathrm{X}_{2} \mathrm{Y}_{2}\right)$ and 2 cross-lag correlations $\left(\mathrm{X}_{1} \mathrm{Y}_{2} \mathrm{Y}_{1} \mathrm{X}_{2}\right.$; Kenny, 1975). The cross-lag analysis assesses the strength of the relationship between two constructs across time (correlations $\mathrm{X}_{1}$ and $\mathrm{Y}_{2}$, and $\mathrm{Y}_{1}$ and $\mathrm{X}_{2}$ ) while controlling for measurement error and spuriousness (e.g., by partialling out $Y_{1}$ from the $X_{1}$-and- $Y_{2}$ cross lag correlation; Kenny, 1975). Essentially, cross-lag correlations establish the direction of causal relationships between two variables over time more reliably than standard correlations. For cross-lag analyses, Kenny (1975) recommended deleting any cases with missing data. However, to avoid substantially reducing an already small sample size, missing data was imputed by AMOS using maximum likelihood estimates (Arbuckle, 2010).

\subsection{Results and discussion}

Table 14 displays the descriptive statistics and pre-post treatment psychometric changes for criminal risk and anger outcomes.

Table 14. Pre and post-treatment VRS risk (dynamic, static, total), STAXI trait anger, and STAXI anger control

$\begin{array}{ccccc}\text { Pre } & \text { Post } & & & \\ M(\mathrm{SD}) & M(\mathrm{SD}) & t & d f\end{array}$

\begin{tabular}{lccccc}
\hline Dynamic Risk & $43.4(7.9)$ & $39.9(7.9)$ & $4.9 * * *$ & 42 & .75 \\
Static Risk & $13.4(2.8)$ & $13.3(2.8)$ & 0.8 & 42 & .12 \\
Total Risk & $57.1(9.2)$ & $53.3(9.2)$ & $4.3^{* * *}$ & 42 & .65 \\
Trait Anger & $17.9(5.4)$ & $15.6(4.0)$ & $3.5^{* *}$ & 41 & .56 \\
Anger Control & $22.2(5.2)$ & $24.3(4.9)$ & $2.7 * *$ & 41 & .43 \\
\hline Note. $* * * p<.001, * * p<.01$ & & &
\end{tabular}




\subsubsection{Pre and post-treatment change in VRS static, dynamic and total risk}

Total VRS scores are interpreted with reference to Wong and Gordon's (2006) risk bands (VRS total scores $<35=$ low, $\geq 35 \leq 50=$ medium, $>50=$ high). Given the high static risk of offenders in the sample, it is unsurprising that both pre and post-treatment total VRS scores met the high-risk cut-off for clinician-rated dynamic risk. As would be expected because static risk is based on relatively unchangeable criminal history variables, total static VRS scores did not change significantly following treatment. In contrast, a medium effect size was observed for dynamic and total risk scores, which significantly decreased following treatment, indicative of treatment gain.

\subsubsection{Pre and post-treatment change in STAXI trait anger and anger control}

For interpretation, mean raw scores are compared to the STAXI-2 normative scores obtained from non-offender males (Trait Anger: $M=18.4, S D=5.42$; Outward anger control: $M=23.5, S D=5.0$ : STAXI-2 Manual; Spielberger, 1999, p.10-11). A comparison of the means in Table 14 with the STAXI normative data shows that before, and particularly after treatment, offenders self-reported less trait anger than non-offender males. Self-reported ability to control the physical or verbal expression of anger was slightly lower than the normative sample pre-treatment, but slightly higher than the normative sample post-treatment; though offender data at both time points was still within the normal range. The direction of changes in trait anger and anger control indicate treatment gain: trait anger significantly decreased, and anger control significantly increased following treatment, both with medium effect sizes. 


\subsubsection{Pre-treatment correlations}

Interpretation of high and low outcome scores vary for each cognitive impulse control task, VRS dynamic risk, STAXI anger, and anger control (e.g., high raw scores on the IGT represent good decision-making whilst high raw scores on the CWIT represent poor performance). Table 15 presents a guide for interpreting the direction of outcome scores for the cognitive impulse control tasks, VRS dynamic criminal risk and STAXI anger outcomes.

Table 15. Interpretive guide for direction of impulse control, dynamic criminal risk and anger outcomes

\begin{tabular}{lll}
\hline Outcome & High score & Low score \\
\hline IGT Total net & Less impulsive & More impulsive \\
CWIT Inhibition & More impulsive & Less impulsive \\
CWIT Inhibit/Switch & More impulsive & Less impulsive \\
CTMT Trail 4 & More impulsive & Less impulsive \\
CTMT Trail 5 & More impulsive & Less impulsive \\
CPT Hit Reaction Time & More impulsive & Less impulsive \\
CPT Commission errors & More impulsive & Less impulsive \\
SSRT & More impulsive & Less impulsive \\
VRS dynamic criminal risk & Higher criminal risk & Lower criminal risk \\
STAXI Trait anger & More anger & Less anger \\
STAXI Anger control & Better anger control & Worse anger control \\
\hline
\end{tabular}


Pre-treatment correlations between cognitive impulse control outcomes, VRS dynamic risk, STAXI trait anger and anger control are displayed below in Table 16. Bonferroni correction was applied to control for Type 1 error $(p<.002)$.

Table 16. Pre-treatment correlations between cognitive impulse control, VRS dynamic criminal risk, STAXI trait anger and STAXI anger control

\begin{tabular}{lccc}
\hline $\begin{array}{c}\text { Cognitive impulse control } \\
\text { outcome }\end{array}$ & VRS dynamic risk & STAXI trait anger & $\begin{array}{c}\text { STAXI anger } \\
\text { control }\end{array}$ \\
\hline IGT Total net & -.22 & -.29 & .27 \\
CWIT Inhibition & .01 & -.02 & -.13 \\
CWIT Inhibit/Switch & .01 & -.17 & .01 \\
CTMT Trail 4 & .07 & -.07 & -.13 \\
CTMT Trail 5 & .02 & -.08 & -.29 \\
CPT Hit Reaction Time & .12 & -.01 & .15 \\
CPT Commission errors & -.01 & -.05 & -.35 \\
SSRT & -.23 & -.03 & .04 \\
\hline
\end{tabular}

The pre-treatment correlations did not empirically support the hypothesised associations between pre-treatment cognitive impulse control, dynamic criminal risk, trait anger and anger control: the relationships were typically minimal and non-significant. In other words, the cognitive impulse control abilities an offender possessed before treatment had little bearing on pre-treatment, clinician-rated dynamic criminal risk.

\subsubsection{Cross-lag correlations}

Although cognitive impulse control was mostly unrelated in a cross-sectional manner to criminal risk, anger experience and anger control before treatment, cross-lag 
correlations were computed to establish the directionality and size of relationships over time. Because dynamic criminal risk and trait anger decreased significantly, and anger control significantly increased following treatment, I wanted to establish whether pretreatment cognitive impulse control was predictive of post-treatment change. Three groups of cross-lag correlations (standardised coefficients) are presented separately according to each cognitive impulse control outcome ${ }^{16}$ in relation to: 1) VRS dynamic risk, 2) STAXI trait anger, and 3) STAXI anger control. Significant cross-lag correlations are indicated by: $* * * p<.001, * * p<.01, * p<.05$

\subsubsection{Cognitive impulse control and VRS dynamic risk}

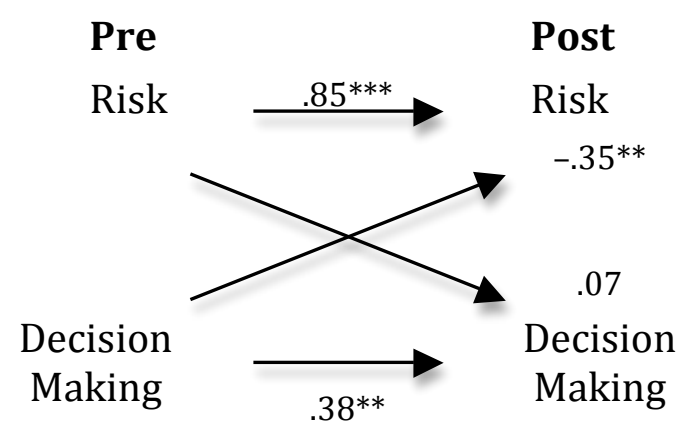

Figure 3. IGT Total Net (good decks-bad decks) and dynamic risk

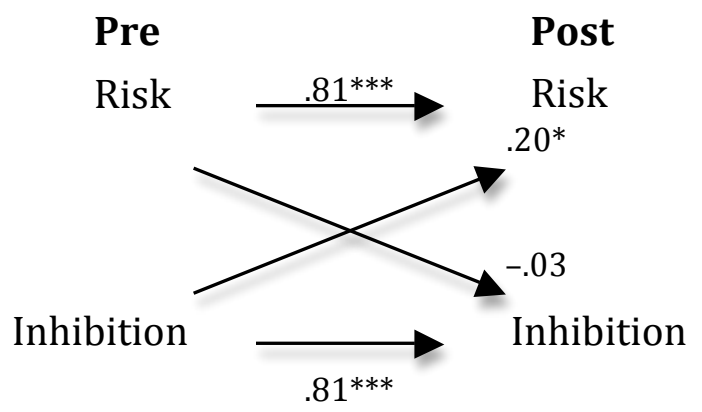

Figure 4. CWIT Inhibition raw completion time and dynamic risk

\footnotetext{
${ }^{16}$ Note: IGT $=$ Iowa Gambling Task CWIT $=$ Colour-Word Interference Test CTMT $=$ Comprehensive Trail-making Test, $\mathrm{CPT}=$ Continuous Performance Test.
} 


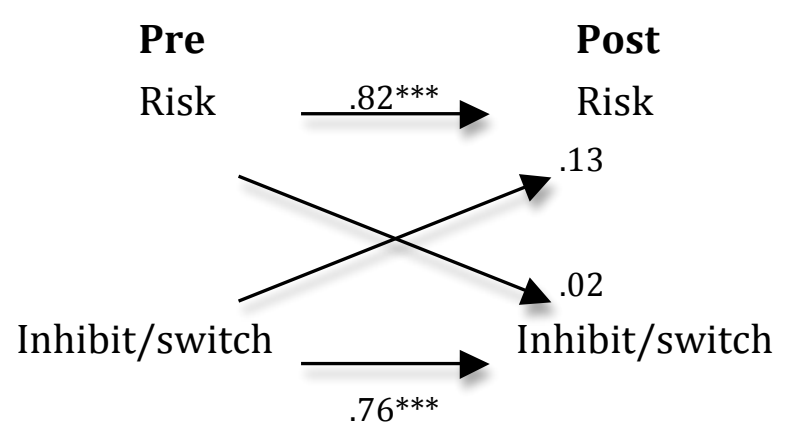

Figure 5. CWIT inhibition/switching raw completion time and dynamic risk

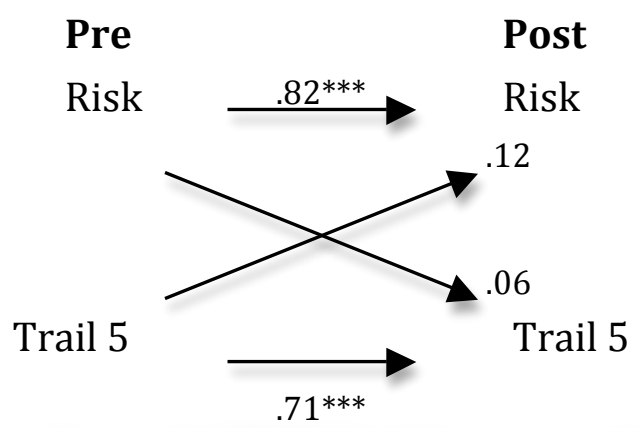

Figure 7. CTMT Trail 5 raw completion time and dynamic risk

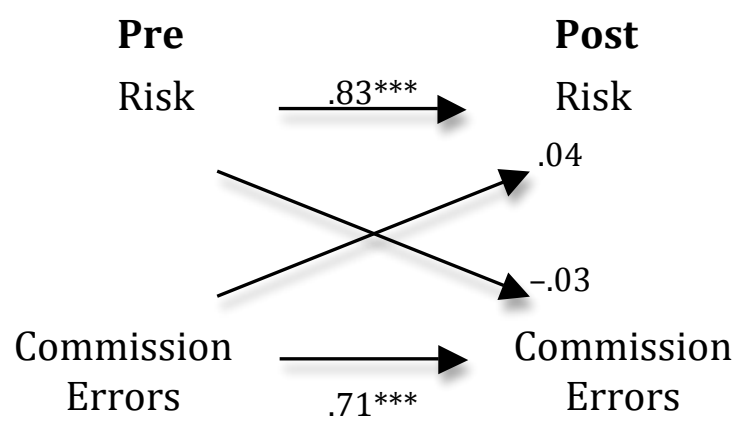

Figure 9. CPT raw commission errors and dynamic risk

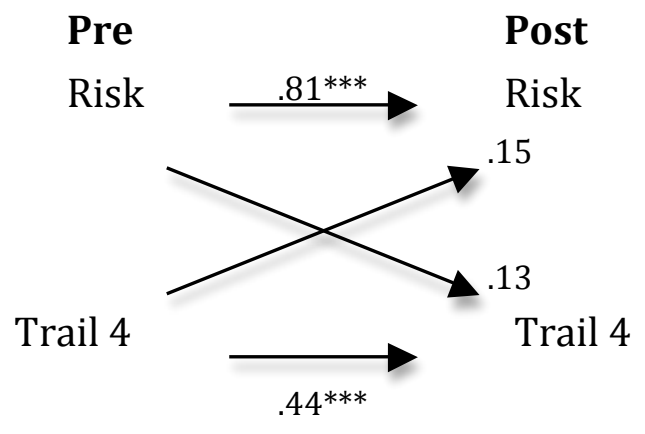

Figure 6. CTMT Trail 4 raw completion time and dynamic risk

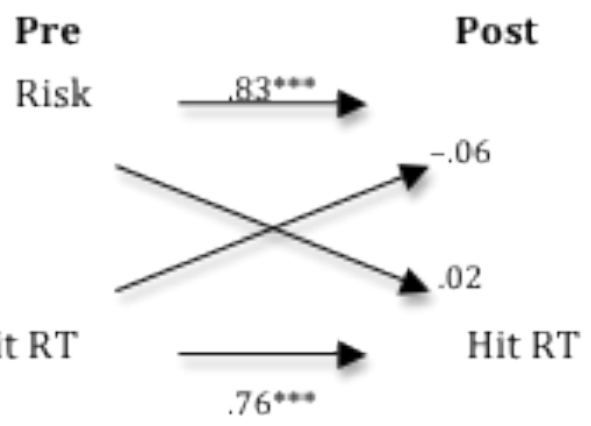

Figure 8. CPT Hit reaction time (ms) and dynamic risk

Pre

Post

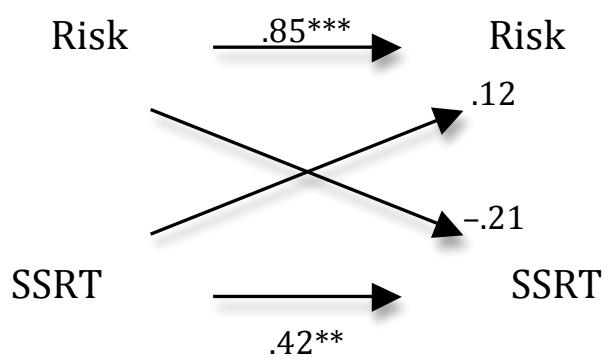

Figure 10. Stop Signal Reaction Time (SSRT) and dynamic risk 


\subsection{Summary of cognitive impulse control and VRS dynamic risk}

The pre-treatment cognitive impulse control variables that predicted posttreatment dynamic criminal risk were IGT decision-making (Figure 3) and CWIT inhibition (Figure 4). That is, greater pre-treatment capacity for making decisions based on options with the best long-term gain was associated with lower post-treatment dynamic criminal risk. In addition, greater difficulty resolving cognitive interference from habitual but unhelpful thoughts was associated with higher dynamic criminal risk following treatment.

\subsubsection{Cognitive impulse control and STAXI trait anger}

Pre

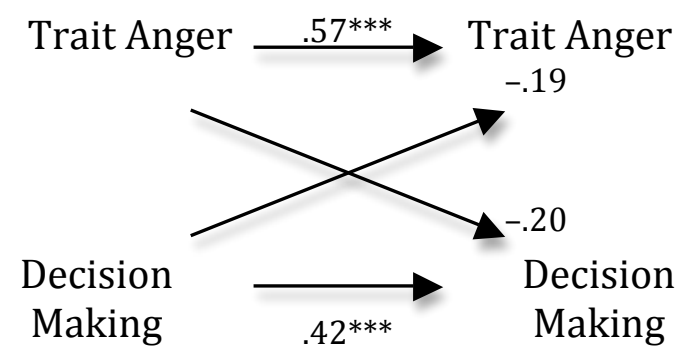

Figure 11. IGT Total Net (good decks bad decks) and Trait anger

Pre Post

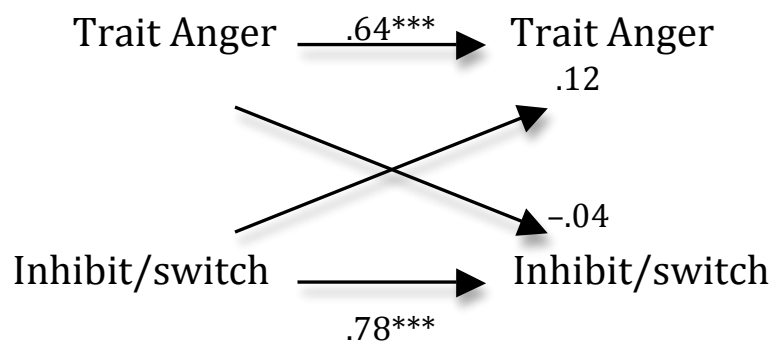

Figure 13. CWIT inhibition/switching raw completion time and Trait anger
Pre Post

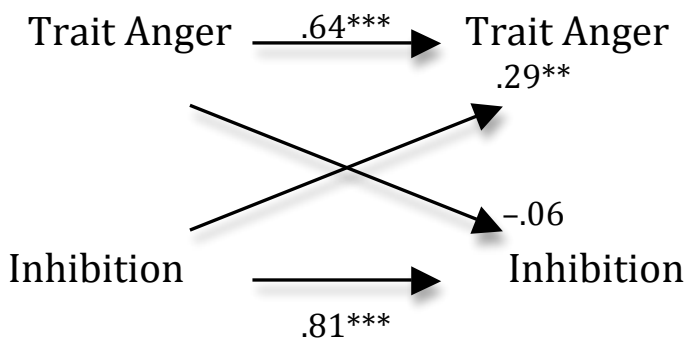

Figure 12. CWIT Inhibition raw completion time and Trait anger

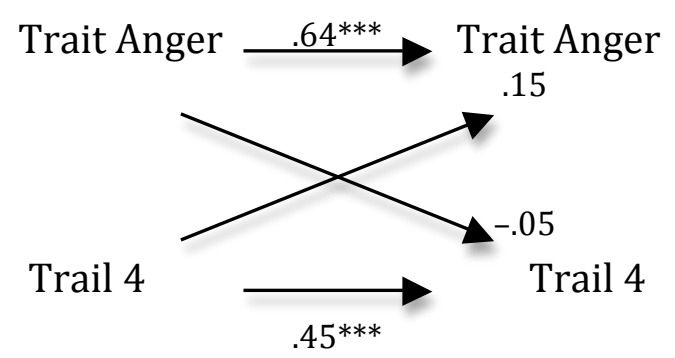

Figure 14. CTMT Trail 4 raw completion time and Trait anger 


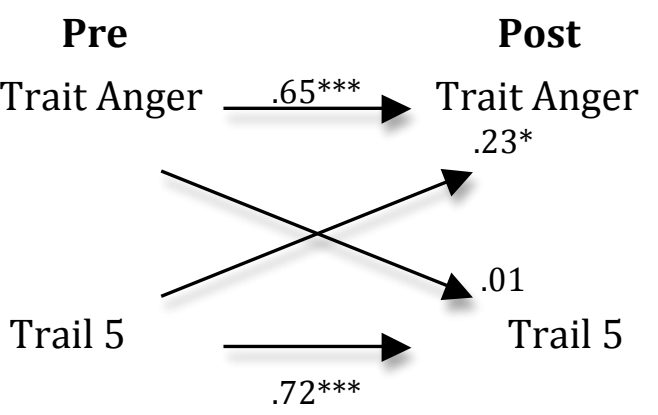

Figure 15. CTMT Trail 5 raw completion time and Trait anger

\section{Pre}

Post

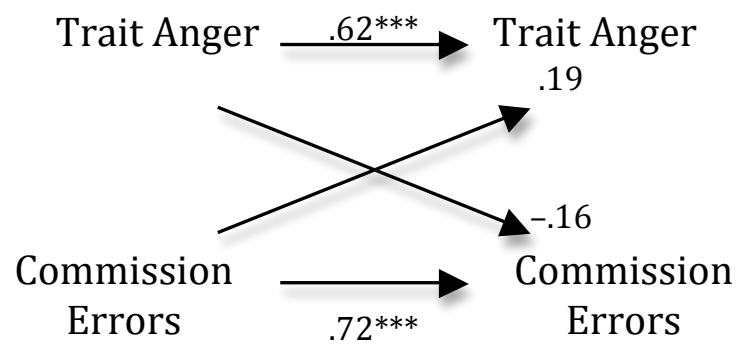

Figure 17. CPT raw commission errors and Trait anger
Pre Post

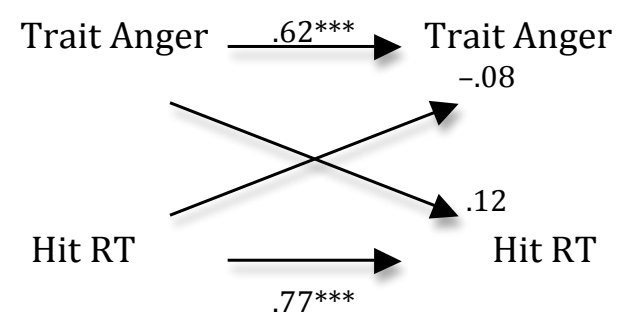

Figure 16. CPT Hit reaction time (ms) and Trait anger

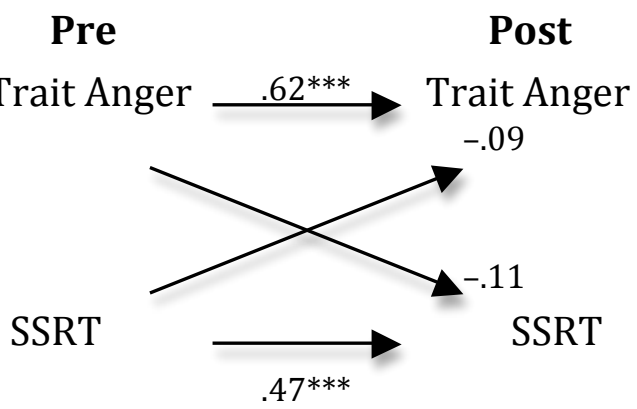

Figure 18. Stop Signal Reaction Time (SSRT) and Trait anger

\subsection{Summary of cognitive impulse control and STAXI trait anger}

The pre-treatment cognitive impulse control variables that significantly predicted post-treatment trait anger were CWIT inhibition (Figure 12) and Trail 5 set shifting on the CTMT (Figure 15). Specifically, greater pre-treatment difficulty with resolving cognitive interference from habitual but unhelpful thoughts, and greater cognitive inflexibility were associated with higher trait anger following treatment. 
Pre

Post

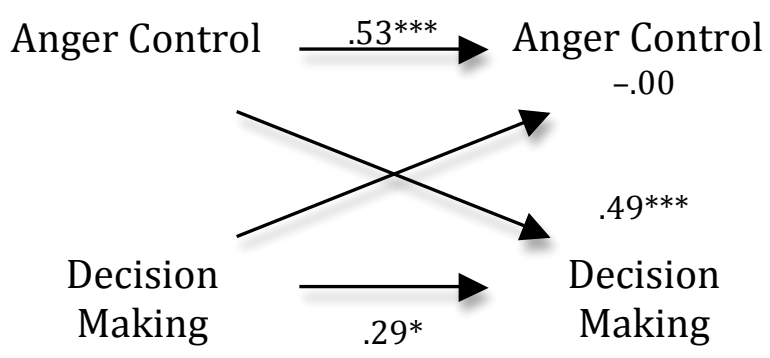

Figure 19. IGT Total Net (good decks bad decks) and Anger control

Pre

Post

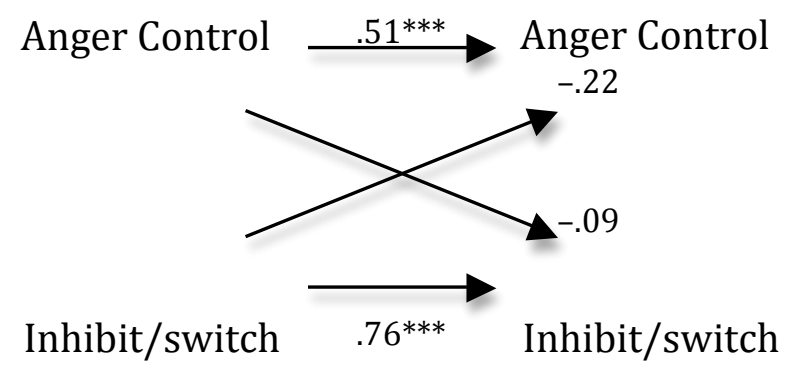

Figure 21. CWIT inhibition/switching raw completion time and Anger control

Pre

\section{Post}

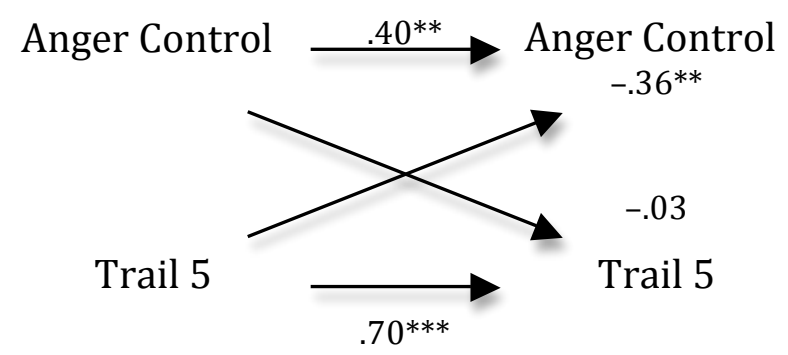

Figure 23. CTMT Trail 5 raw completion time and Anger control
Pre

Post

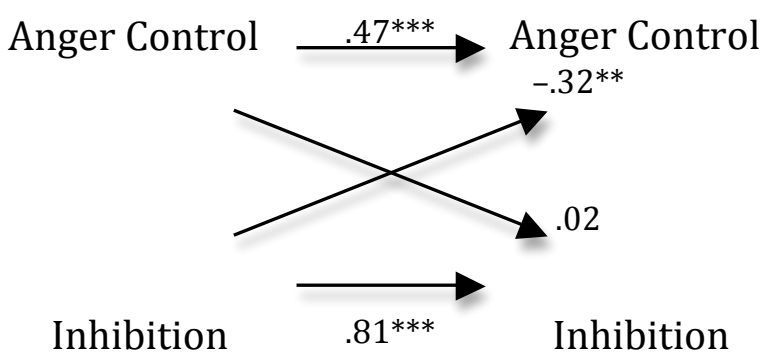

Figure 20. CWIT Inhibition raw completion time and Anger control

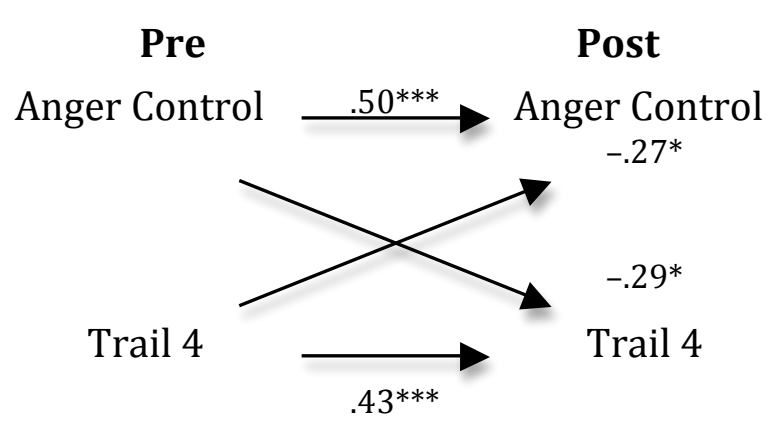

Figure 22. CTMT Trail 4 raw completion time and Anger control

Pre

Post

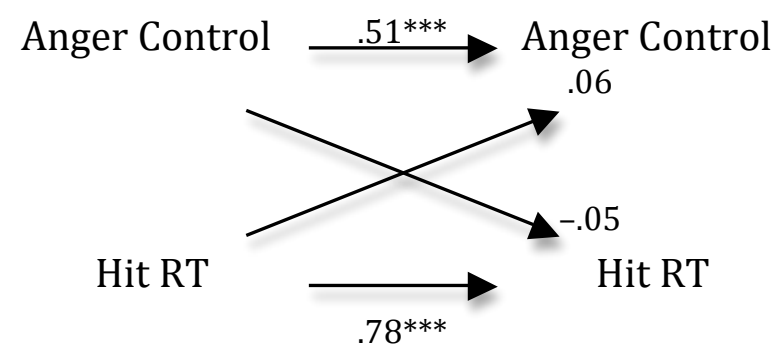

Figure 24. CPT Hit reaction time (ms) and Anger control 


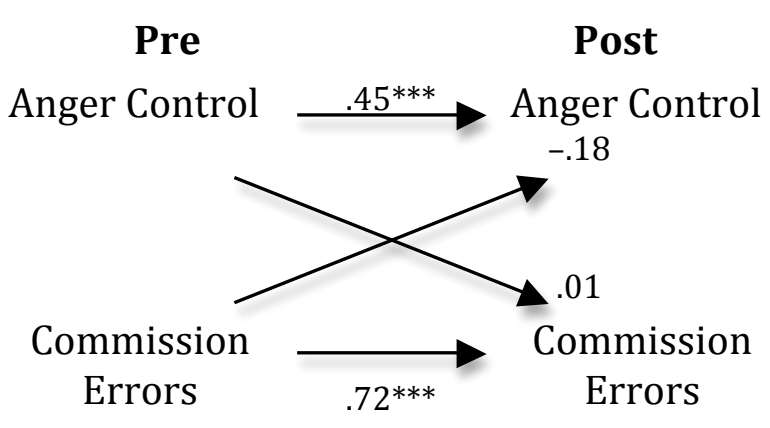

Figure 25. CPT raw commission errors and Anger control
Pre Post

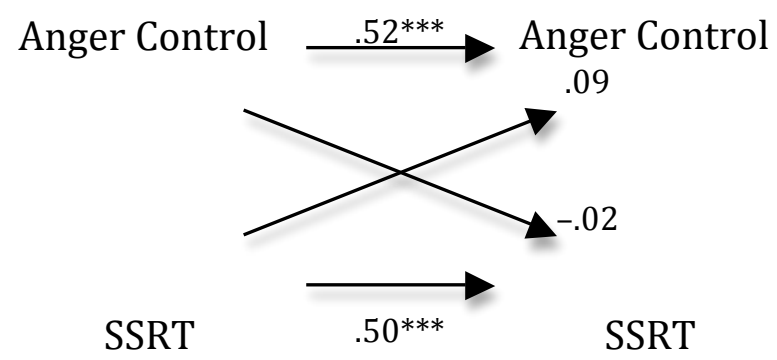

Figure 26. Stop Signal Reaction Time (SSRT) and Anger control

\subsection{Summary of cognitive impulse control and STAXI anger control}

The pre-treatment cognitive impulse control variables that significantly predicted post-treatment anger control were CWIT inhibition (Figure 20), Trail 4 (Figure 22) and Trail 5 (Figure 23) set shifting on the CTMT. For CWIT inhibition and Trail 5 set shifting, the directions of the relationships were such that poorer pre-treatment ability to resolve cognitive interference from habitual but unhelpful thoughts, and poorer cognitive flexibility predicted lower (i.e., poorer) post-treatment anger control. Put another way, this means that better pre-treatment impulse control predicted better post-treatment anger control. Interestingly, there was a reciprocal relationship for Trail 4 set shifting, whereby poorer pre-treatment set shifting both predicted, and was predicted by poorer anger control. In addition, a significant predictive relationship emerged whereby better pretreatment anger control predicted more advantageous post-treatment decision-making. I 
now turn to a more detailed discussion of interpretations and implications of the cross-lag relationships.

\subsection{General discussion}

The overarching aim of this study was to investigate whether there was any empirical support for specific aspects of Moffitt's (1993) LCP theory. First, I wanted to test the hypotheses that poorer pre-treatment cognitive impulse control would be significantly associated with higher pre-treatment dynamic violence risk, higher trait anger and lower anger control. In direct contrast to Moffitt's prediction that cognitive impulse control contributes to criminal risk, I found negligible, non-significant relationships between all cognitive impulse control outcomes and pre-treatment, clinician-rated dynamic criminal risk. Regarding pre-treatment cognitive impulse control, trait anger and anger control; the overall picture was also one of little association. Taken together, the pre-treatment correlations challenged the theoretical notion that cognitive impulse control abilities assessed at any one point in time would account for substantial variance in an offender's criminal risk. The pre-treatment correlations also partly challenged Moffitt's prediction that co-occurring inhibitory deficits and negative emotionality would be elevated in LCP offenders. Although not directly tested in this study, previous research suggests that the majority of New Zealand's high-risk offenders are typically on the LCP path (Wilson, 2004). Therefore, if cognitive impulse control deficits and negative emotionality remain as stable in adulthood as Moffitt's LCP theory would predict, significant correlations of greater magnitude would have been observed between cognitive impulse control, trait anger and anger control. 
However, that is not to say that cognitive impulse control is not an important variable to consider in risk assessments and treatment interventions: pre-treatment decision-making and some perceptual impulse control—but not motor impulse control— outcomes predicted post-treatment risk and anger outcomes. Together, the pre-treatment and cross-lag correlations suggested that whilst cognitive impulse control might not be related to other risk-related offender characteristics at the beginning of treatment, decision-making and perceptual impulse control abilities influence the capacity for positive progress during treatment. In other words, cognitive impulse control represents an individual-level characteristic that could influence engagement and progress in treatment (Andrews \& Bonta, 2006). I now discuss more specifically how cognitive impulse control could facilitate or block change in criminal risk and emotion regulation over the course of treatment, based on the directions of cross-lag relationships observed in this study.

\subsubsection{Decision-making, inhibition and dynamic violence risk}

On average, dynamic violence risk decreased significantly post-treatment. Better pre-treatment decision-making and inhibition predicted lower post-treatment dynamic criminal risk. Together, these relationships suggest that treatment progress may be limited for offenders who begin treatment with poorer cognitive capacity for generating multiple action plans - particularly regarding response options that are consistent with longer-term goals - and resisting cognitive distraction from old ways of thinking that are at odds with new goals. This finding is consistent with previous studies suggesting that the integrity of particular cognitive functions can account for variability in treatment response (e.g., Aharonovich, Nunes \& Hasin, 2003; Bauer, 2001; Fishbein et al., 2006). However, 
studies investigating these relationships to date typically focus on treatment response for substance use interventions, not on correctional treatment responses of adult offenders.

The criminogenic potential of poor decision-making and inhibitory ability is not new in the correctional literature. However, poor decision-making and inhibitory ability are typically conceptualised as treatment targets not as individual-level factors that could affect an offender's ability to engage in, and respond to treatment per se. Because pretreatment criminal risk was not significantly associated with pre-treatment decisionmaking and inhibitory ability, and because decision-making and inhibitory abilities were generally intact (see Chapter 5), the findings of this research support the conceptualisation of cognitive impulse control functions as responsivity factors, more so than factors that make substantial, direct contributions to dynamic risk. In other words, cognitive impulse control might not directly maintain criminal risk, but it could maintain risk indirectly by precluding or limiting the capacity for change (Ward, Day, Howells \& Birgden, 2004).

It makes intuitive sense that decision-making and inhibition are critical facilitators of change because progress in correctional programmes like the STURPs, requires offenders to "be cognizant of and responsive to potential negative consequences of their behavior, inhibit inappropriate behavioral responses, and understand and act on the benefits of deliberate and cautious decision making" (Fishbein et al., 2009, p.420). Although further investigation is required to explore this suggestion, decision-making and inhibitory abilities might be equally important for maintaining any changes offenders make in treatment. Presumably, mentally projecting what current behaviour will mean for longer-term outcomes, and inhibiting any behaviours that are inconsistent with desired 
long-term outcomes are important abilities for avoiding high-risk behaviours or approaching pro-social alternatives.

The predictive links between decision-making, inhibitory abilities and change in criminal risk highlight the importance of identifying weaknesses in these cognitive functions. The assessment of decision-making and inhibition ability in the risk assessment process would help to identify offenders whose criminal risk is directly maintained by weaknesses in these areas. However, of greater importance is assessment of decision-making and inhibitory ability in the context of formulations about specific responsivity factors for each offender. Without acknowledging the possibility that suboptimal cognitive impulse control could operate as a treatment target and a responsivity issue, clinicians run the risk of providing help in these areas to offenders who need it most to reduce criminal risk; but who will benefit the least without first targeting their fundamental cognitive weaknesses.

\subsubsection{Decision-making, inhibition, set shifting, trait anger and anger control}

Trait anger significantly decreased, and anger control significant increased following treatment. Lower post-treatment trait anger and better anger control was significantly predicted by better pre-treatment ability to resolve cognitive interference from habitual but unhelpful thoughts, and flexibly shift attention between different thoughts. The directions of these relationships suggested that being able to alter how frequently and intensely anger is generated, and gaining better control over the expression of anger, draws on intact ability to resist cognitive interference and shift attention. In other words, cognitive impulse control skills play an integral role in improving emotion 
regulation at the stage of emotion generation and at the later stage of controlling the expression of emotion (Gross \& Thompson, 2007).

This finding takes preliminary steps toward explaining how variation in cognitive impulse control might affect other areas of functioning that contribute to criminal risk. Specifically, the results of this study are consistent with previous work implicating intact cognitive processing for using mental strategies that attenuate anger; strategies offenders with poorer inhibition and attention shifting will be less able to implement effectively (e.g., Bunge, Ochsner, Desmond, Glover \& Gabrieli, 2001; Ochsner, Ray, Cooper, Robertson, Chopra, Gabrieli \& Gross, 2004).

It is unclear from this study if offenders who made the largest post-treatment reductions in trait anger did so by using mental strategies to reduce angry feelings. However, the findings at least suggest that offenders who begin treatment with greater inhibition and cognitive flexibility will be the offenders who have the greatest capacity for implementing and practicing anger management strategies. On the flipside, offenders who struggle with resolving cognitive interference and shifting attention away from external or internal sources of anger might only be able to learn about emotion regulation strategies at a superficial level; whilst success in implementing and practicing those strategies will be limited.

Two relationships between cognitive impulse control and anger control were inconsistent with hypotheses. First, better pre-treatment anger control predicted more advantageous post-treatment decision-making but the reverse was not true. Intuitively, the reverse relationship makes more sense: people who are better able to make choices that maximise beneficial longer-term outcomes would be more likely to control the 
expression of anger to avoid the likely negative consequences that would follow should anger be expressed. However one explanation is that even if offenders begin treatment possessing the cognitive skills to forecast which behaviours will secure beneficial longerterm outcomes according to pro-social standards, this may not guarantee the best option is chosen. That is, some offenders may deliberately choose not to control anger because of the desirable consequences of anger expression, such as coercing change in another person's behaviour (Fischer \& Roseman, 2007), or re-gaining control in interpersonal interactions perceived as threatening and hostile (Seager, 2005).

Decision-making itself significantly improved following treatment; although it is unclear how, or if, the treatment intervention per se contributed to this change. Nevertheless, another possibility is that offenders already able to exert substantial effort to control the expression of anger were the same offenders with the cognitive capacity for learning how to become more proficient at long-term focused thinking more generally; thus explaining more advantageous post-treatment decision-making. From this view, selfreported anger control may capture generalised cognitive control more so than emotionspecific control skills per se. The second unexpected relationship, in which set shifting on the CTMT both predicted, and was predicted by anger control, supports the notion that cognitive control and anger control draw on the same, general control function.

\subsection{Summary}

In summary, the predictive links in this study speak to cognitive impulse control skills as representing possible treatment targets for risk reduction in their own right, but more so as possible facilitators of change in other areas of criminal risk: specifically, emotion regulation. Sub-optimal decision-making and perceptual impulse control are 
likely to represent potential barriers to treatment progress. Accordingly, it is important for risk assessment and treatment formulation that clinicians take cognitive impulse control skills into consideration to establish how functioning in these areas is likely to affect treatment responses for individual offenders. 


\section{Chapter 8}

\section{General Discussion}

\subsection{Revisiting thesis aims and chapter overview}

Understanding the aetiology and maintenance of criminal risk and providing effective treatment interventions relies on a theoretical understanding of individual risk factors and ideally, their relationship to one another (Ward, Polaschek \& Beech, 2005). Although impulsivity is an important risk factor and treatment target, our current understanding of impulsivity in a forensic setting is atheoretical. As a consequence, risk assessment and treatment interventions are often guided by the inference of cognitive failures to explain impulsive-looking behaviour. However, empirical support for the validity of this inference is inconsistent. Without clear theoretical guidelines for organising and understanding the many definitions of impulsivity, it remains unclear precisely what the term impulsivity means, and most importantly, how impulsivity explains variation in criminal risk across the lifespan. The effectiveness of interventions assisting offenders to become better regulators of their impulses will be limited if not informed by theory regarding the causal mechanisms worthy of treatment attention.

To develop a better theoretical understanding of the contribution of impulsivity to criminal risk in adult offenders, the overarching objective of this thesis was to investigate impulsivity from a cognitive approach given the conceptual and methodological limitations of overreliance on personality and behavioural assessments of impulsivity. To this end, this study adopted a neurocognitive framework because it offered a theoretical underpinning of the concept of impulsivity that could be used to assess key domains of 
cognitive impulse control. Using this framework, the specific aims of this thesis were to investigate: 1) the patterns and prevalence of cognitive impulse control impairment, 2) whether cognitive impulse control functions have the capacity to change following a cognitive-behavioural treatment intervention, and 3) whether cognitive impulse control is related to other important risk-related outcomes.

Applying the neurocognitive framework to the assessment of cognitive impulse control in a sample of high-risk offenders, I found that: 1) mechanisms of cognitive impulse control are important to consider in risk assessment and intervention even if clinical impairment is not prevalent; 2) some mechanisms of cognitive impulse control improved, though typically not significantly following a cognitive-behavioural intervention, and 3) there were some predictive relationships between cognitive impulse control and the amount of progress made on other risk-relevant outcomes by the end of treatment; thus, improving impulse control could improve other dynamic risk factors.

The findings make unique theoretical contributions in three important ways. First, they challenge the assumption inherent to criminal risk assessment tools that dysfunctional behaviour that appears ill planned and rapidly executed is best explained by chronic reflective system weaknesses. Explaining behaviour in this way was not empirically supported, suggesting that cognitive failure is not the only way to conceptualise causal mechanisms of impulsivity in criminal risk assessments. Second, small amounts of post-treatment improvement suggest that cognitive impulse control has the potential to be strengthened and developed further in adulthood. This challenges the central tenet of developmental theories of antisocial behaviour that stability in cognitive impulse control impairment maintains criminal risk for LCP offenders. 
Lastly, a theoretical understanding of the interplay between different risk factors and how change on one risk factor could engender change on others is limited (Polaschek, 2010; Ward, Polaschek \& Beech, 2005). The findings of this study fill one part of this theoretical gap by demonstrating that pre-treatment cognitive impulse control—specifically perceptual impulse control—predicted post-treatment improvements in anger-related outcomes.

The theoretical implications are discussed in further detail below alongside implications for risk assessment, clinical formulation and treatment intervention. The limitations of the study are outlined, highlighting important directions for future research, in order to continue theory development and further understanding of the contribution of impulsivity to criminal risk.

\subsection{Theoretical implications}

\subsubsection{Chronically weak reflective systems: Lack of empirical support}

The Violence Risk Scale (VRS), its sexual offender version (VRS-SO) and other common risk assessment instruments that include an impulsivity item are based on the behavioural approach: current and historical behavioural evidence is evaluated to judge whether dysfunctional impulsivity contributes to an offender's criminal risk. In both the violent and sexual offender versions, VRS rating criteria operationalise impulsivity as: "spur of the moment occurrences in which the individual reacts without pausing to consider the costs or consequences of negative behaviour" and "the individual tends not to consider relevant information before reacting" (VRS Manual; Wong \& Gordon, 2000, p.64). If impulsivity is a "characteristic of the individual's mode of behaviour", a 
maximum score indicative of clinically significant impulsivity is warranted (VRS Manual; Wong \& Gordon, 2000, p.64).

Based on these criteria, a causal role is attributed to stable reflective system weakness that operates like a trait. That is, behaviour is predominantly driven by the immediate needs of the impulsive system because regulation of these needs by the reflective system typically fails. However, clinical impairments in decision-making (reacting before considering consequences), perceptual and motor impulse control (reacting before relevant information is considered), were not empirically supported in the present study. Contrary to expectation, the majority of offenders displayed intact decision-making that reflected consideration of beneficial long-term outcomes and avoidance of punishment. Offenders also displayed the ability to inhibit dominant but incorrect responses, and demonstrated strategic approaches to cognitive tasks that maximised response accuracy over speed. Cognitive inflexibility, or difficulty shifting attention between multiple thoughts at once, was most impaired in this study, consistent with other adult offender studies (e.g., Crump, 2005; Hancock, Tapscott \& Hoaken, 2010; Lapierre, Braun \& Hodgins, 1995). However, to my knowledge, none of the well-known criminal risk instruments described in Chapter 1 acknowledge the possible causal role of impaired cognitive flexibility in formulations of impulsivity. Despite offenders only demonstrating, on average, minor cognitive impulse control impairments on cognitive tasks, VRS and VRS-SO pre-treatment assessments identified $89 \%$ of offenders in this study as requiring therapeutic intervention for dysfunctional impulsive behaviour. Furthermore, correlations between pre-treatment cognitive impulse control and dynamic risk ratings on the VRS were mostly negligible and non-significant. 
Thus, nearly the whole sample was judged to have chronically weak reflective skills. For a small proportion of offenders in the present sample who displayed clinically impaired cognitive impulse control both before and after the treatment intervention, the assumption of trait-like reflective impairments was empirically supported. But the degree to which those impairments could explain criminal risk was limited. For a much larger proportion of offenders, the assumption of stable reflective weaknesses was not empirically supported; either clinical impairment was not observed at all, or small improvements following treatment determined that impairment was not stable.

Taken together, clinical inference of stable, trait-like failures in cognitive impulse control as an explanation for impulsive-looking behaviour could lead to erroneous risk formulations and misdirection of treatment intervention efforts. Without assessing impulse control via cognitive measures, clinicians are limited in their ability to differentiate offenders for whom poor cognitive impulse control represents a significant treatment need, from offenders who have intact cognitive impulse control skills. The mismatch between low-level cognitive impairment and strong indications of treatmentworthy dysfunctional impulsive behaviour on the VRS and VRS-SO prompts speculation about what else is captured in criminal risk ratings of impulsivity. I argue that the types of dysfunctional impulsive behaviours captured within VRS or VRS-SO impulsivity are underpinned by the co-existence of two related, but potentially dissociable types of impulsivity: cognitive and personality impulsivity. 


\subsubsection{Different explanations for dysfunctional impulsive behaviour: Cognitive versus}

personality impulsivity

According to Moffitt's (1993) LCP theory, inherited or acquired compromise to neuropsychological health determines that LCP offenders have difficult temperaments, which manifest as disinhibited behaviour and an angry, irritable disposition (Moffitt, 1993). The disinhibitory component of difficult temperament is proposed to be underpinned by: 1) inhibitory deficits, 2) sensation seeking, and 3) high activity level (Moffitt, 1993). People with inhibitory deficits behave without thinking or realising the risks involved, whereas people high on sensation seeking with a high need for activity willingly approach risky behaviour because they desire thrill and excitement (Eysenck, Pearson, Easting \& Allsopp, 1985). A large body of research shows that outcomes from measures capturing both inhibitory deficits and sensation seeking dimensions of impulsivity share some variance but mostly the two do not overlap (e.g., Cyders \& Coskunpinar, 2012; Dolan \& Fullam, 2004; Evenden, 1999; Magid, MacClean \& Colder, 2007).

The mismatch between VRS/VRS-SO impulsivity ratings and low-level cognitive impairment found in this research may be explained by the co-existence of another type of impulsivity that more fully accounts for the types of dysfunctional behaviours captured in VRS/VRS-SO impulsivity ratings. Specifically, one type of impulsivity relates to cognitive mechanisms of impulse control, as measured in this research (i.e., decisionmaking, perceptual and motor impulse control). Another type of impulsivity relates to sensation seeking and high activity level—personality-based factors — that were not directly measured in this research. The crucial difference between the two proposed types 
of impulsivity relates to: failing to develop and act on avoidance goals, versus strong approach goals for risky behaviour (Cochran \& Tesser, 1996; Elliot \& Thrash, 2002).

I propose that the cognitive type of impulsivity is aligned with Moffitt's inhibitory deficit sub-component of disinhibition, and the types of cognitive processes assessed in the present study. For offenders with cognitive impulsivity, dysfunctional behaviour will be expressed because of poor cognitive ability to develop avoidance goals and poor capacity for inhibiting behaviour that leads to undesirable outcomes. Consequently, behaviour is driven by automatic approach goals developed from previous learning experiences, even if that behaviour is at odds with longer-term goals, or society's goals (Baumeister, Heatherton \& Tice, 1994; Carver \& Scheier, 1981; Chen \& Bargh, 1999; Mann, Webster, Schofield \& Marshall, 2004; Ward, \& Hudson, 2000). In this sample, cognitive impulse control was mostly intact and the pre-treatment correlations between cognitive impulse control outcomes and dynamic risk were small, and largely nonsignificant. This suggests that enduring clinical impairments in cognitive impulse control, which function like a trait, may only explain the types of dysfunctional behaviour captured in instruments like the VRS/VRS-SO for a small proportion of high-risk offenders.

I propose that personality impulsivity is aligned with Moffitt's sensation seeking and high activity level sub-components of disinhibition, and with a personal preference for risk and excitement. For offenders with personality impulsivity, dysfunctional behaviour will be expressed because of deliberate approach to risk-taking behaviour because of the thrill and excitement such behaviour provides. Given the mismatch between low-level cognitive impulse control impairment and high VRS/VRS-SO 
impulsivity ratings, personality impulsivity may explain the types of dysfunctional behaviour captured in instruments like the VRS/VRS-SO for a relatively larger proportion of high-risk offenders than cognitive impulsivity.

Despite recognition of the important difference between avoidance of an outcome and deliberately approaching an outcome in other psychological literatures (e.g., Eysenck, Pearson, Easting \& Allsopp, 1985), it seems the two may be confounded in the forensic literature. The mismatch between the low prevalence of cognitive impulsivity and high VRS/VRS-SO impulsivity suggests that impulsivity is being mislabeled as the product of failed cognitive control when, for a much larger proportion of offenders, impulsivity may be the product of a general disposition for preferring the thrill and excitement that accompanies risky behaviours which have the potential to end badly.

Although personality impulsivity was not directly measured in this study, observations made during the research data collection anecdotally suggested that personality impulsivity was common in the present sample. For example during the gambling task, after realising which decks were good and bad, a number of participants articulated that it was boring to "play it safe" and expressed a desire to try their luck by deliberately making selections from the highest-risk, most disadvantageous deck: Deck A. A sensation seeking explanation might be able to explain the overall favouritism toward Deck D (low loss magnitude, low loss frequency), followed closely by favouritism toward Deck B (high loss magnitude, low loss frequency) observed in the present study. That is, favouritism toward Deck D was driven by the low sensation seekers and Deck B preference was driven by the high sensation seekers: Deck B provided sensation seekers with the thrill of high reward similar to Deck A, but with less 
severe costs. Recently, Buelow and Suhr (2013) have shown that preference for Deck D selections is negatively associated with higher approach motivation (Carver \& White, 1994) and with higher impulsiveness and sensation seeking scores on the ZuckermanKuhlman Personality Questionnaire. In contrast, preference for Deck B selections was positively associated with those variables (Buelow \& Suhr, 2013). Within the context of Buelow and Suhr's findings, preference for Decks D and B could have captured intact decision-making in which choice was guided by consideration of loss magnitude and frequency to ensure beneficial outcomes. But it is also possible that choice preferences reflected individual variation in sensation seeking, or personality impulsivity (Suhr \& Tsanadis, 2007). Support for this possibility was illustrated by inspection of the correlations between total Deck B and D selections: both before and after treatment, the number of selections from each deck were significantly, inversely correlated.

Criminal risk assessment tools vary in whether they acknowledge cognitive and personality impulsivity as explanations for impulsive behaviour. The VRS and VRS-SO make no mention of personality impulsivity (i.e., sensation seeking, high activity level) and therefore appear to exclusively operationalise impulsive behaviour as caused by cognitive impulsivity. On the other hand, the STABLE (Hanson, Harris, Scott \& Helmus, 2007) states that impulsive behaviour can occur because the individual is "easily bored and seeks thrills" (e.g., STABLE scoring guide, p.42).

The differentiation between cognitive and personality impulsivity is theoretically and clinically important for risk assessment as each type may make different contributions to criminal risk. Empirical findings show that impulsivity (i.e., cognitive impulsivity) and sensation seeking (i.e., personality impulsivity) differentially account for 
variance in clinically important outcomes. For example, in the substance abuse literature, Magid, MacClean and Colder (2007) found direct and indirect relationships between sensation seeking and alcohol use. The relationship between sensation seeking and frequency of alcohol use was mediated by the motivation to enhance positive affect, consistent with the notion that high sensation seekers approach risky behaviour to heighten stimulation (Magid, MacClean \& Colder, 2007; Zuckerman, 1994). In contrast, Magid and colleagues found that impulsivity (more closely aligned with cognitive impulsivity) was not related to frequency of alcohol use, but to the use of alcohol as a coping strategy.

Magid and colleagues' study highlights the need for a more nuanced approach to formulating impulsivity. It is important for clinicians to be aware of the theoretically and clinically meaningful differentiation between impulses that cannot be resisted and impulses that are deliberately not resisted (Ogloff, 1997). Without such differentiation, behaviour may be mislabelled and the underlying causal process misattributed to cognitive impulsivity when personality impulsivity is a more likely causal mechanism. Mislabeling —effectively misdiagnosing — will result in a less suitable intervention being offered to the offender. Those with cognitive impulsivity are likely to benefit more from treatment interventions focused on the development of avoidance goals and strengthening the requisite cognitive skills for inhibiting behaviour based on those goals. In contrast, offenders with personality impulsivity are more likely to benefit from treatment interventions focused on finding more adaptive, pro-social means of finding excitement and developing greater tolerance for boredom (Matthies, Philipsen \& Svaldi, 2012; Ward \& Gannon, 2006; Watt \& Vodanovich, 1992). 


\subsubsection{Two non-impulsive types to consider}

In addition to identifying different types of impulsivity in the risk assessment process, it is also important that clinicians are able to identify non-impulsive explanations

for behaviour that looks impulsive. For example, one possibility is that impulsive-looking behaviour is underpinned by more general, rather than impulse control-specific, cognitive impairments. Performance outcomes on the Continuous Performance Test (CPT) and the Stop Signal task indicated that some offenders were impaired in their ability to pay attention and respond consistently. Behavioural observations during the research data collection supported this impairment: a number of participants tried to engage in conversation during the task and explicitly commented that it was difficult to concentrate, particularly towards the end of the research session. One participant completely stopped during the CPT and began playing with buttons on the laptop unrelated to the assessment task. Difficulty remaining focused is consistent with meta-analyses showing that offenders typically perform between .44 and .62 standard deviations below normative comparisons with medium to large effect sizes on executive function tests, with the largest effect sizes for attention measures (Morgan \& Lilienfeld, 2000; Ogilvie, Stewart, Chan \& Shum, 2011).

Identifying offenders with poor sustained attention is crucial in a treatment context, because cognitive weaknesses of this kind represent an important responsivity barrier to treatment engagement (Fishbein, et al., 2009). It is also important for understanding specific mechanisms of impairment. Weaknesses in sustained attention might make successful impulse control more inconsistent, but these weaknesses do not represent an impulse control-specific deficit per se. Poor sustained attention may represent a much 
broader treatment need that should be targeted instead of, or at least before, impulsecontrol specific processes.

Another non-impulsive explanation to consider is when behaviour appears to be impulsive simply because it is the product of well-rehearsed responses that have become automatic from repeated practice over time (Shiffrin \& Schneider, 1977). In this case, criminal risk would not be explained by cognitive or personality impulsivity but by other processes like an antisocial learning history that has reinforced behaviours that are dysfunctional by prosocial standards (Bandura, 1986). For such offenders, no amount of reflection on the consequences of behaviour will necessarily lead to the selection of prosocial options because the most familiar behaviours are antisocial. Therefore, clinical formulation and treatment intervention would be better focused on promoting the benefits of pro-social behaviour, assisting the development of a pro-social behavioural repertoire, and rewarding pro-social behaviour, rather than focusing on cognitive or personality impulsivity.

\subsubsection{Developmental trajectories of cognitive and personality impulsivity}

Although not directly measured in this research, previous research suggests that the majority of high-risk offenders are typically on the LCP path (Wilson, 2004). Moffitt's LCP (1993) theory predicted that both cognitive and personality types of impulsivity contribute to problematic, antisocial behaviour early in life. The other major assumption underlying Moffitt's (1993) LCP theory and other famous criminological theories like Gottfredson and Hirschi's (1990) self-control theory, is one of stability in impulse control impairment across the life course relative to the general, non-offender population. However, empirical evidence challenges the stability assumption by showing 
that there are unique, fluid developmental patterns of self-control over the life span (e.g., Hay \& Forrest, 2006; Higgins, Jennings, Tewksbury \& Gibson, 2009; Ray, Jones, Loughran \& Jennings, 2013). Small improvements in some cognitive impulse control outcomes were observed in the present study, also consistent with the notion of fluidity.

The mean pre-treatment age of offenders in the present study was 32 years. Generally intact cognitive impulse control in this sample - particularly for decisionmaking and inhibition of habitual responses — could mean that decision-making and inhibition abilities were always intact. This explanation cannot be tested without having assessed cognitive impulse control earlier in life for offenders in this sample. Another possibility is that the offenders recruited in this research were LCP offenders who had already made progress in developing impulse control through practice in prison. To attend the STURPs, an offender's security level must be low-medium to minimum (Polaschek \& Kilgour, 2013). Obtaining this type of low internal security risk rating requires consistently good institutional behaviour. However, an alternative possibility based on research of neural development across the lifespan is that, with age, comes further development of neural areas that facilitates better cognitive impulse control (Romine \& Reynolds, 2005). Therefore, it is suggested that the development of cognitive impulse control skills might initially be delayed for LCP offenders compared to the general population, accounting for the relatively more robust relationship between impulsivity and antisocial behaviour during childhood and adolescence than in adulthood. But by adulthood, whilst many LCP offenders may still rank below normative comparisons in absolute ability, intra-individual improvements could occur because the 
neural mechanisms that facilitate better impulse control have 'caught up' developmentally (Miyake \& Friedman, 2012; Piquero \& Moffitt, 2011; Soper, 2001).

Consistent with Moffitt's theory, it is possible that the delay in cognitive impulse control development for LCP offenders begins from inherited or acquired neuropsychological deficits, which are then exacerbated by chaotic, unpredictable developmental environments (e.g., Moffitt, 1993; Piquero, 2001; Piquero \& Tibbetts, 1999; Tibbetts \& Piquero, 1999). Parents of LCP children are less likely to provide developmental environments conducive to modelling, practicing or positively reinforcing impulse control (Moffitt, 1993). Children on the LCP path are also less likely to spend time in social institutions that model and promote the benefits of impulse control, such as the schooling system (Moffitt, Caspi, Harrington \& Milne, 2002). Consequently, opportunities to practice and learn the benefits of impulse control that are ordinarily provided by parents and social structures become limited for children on the LCP path (Moffitt, 1993). As a result, cognitive delay in impulse control development may represent an initiating risk factor for antisocial behaviour by increasing exposure to other risk factors, such as contact with other under controlled peers and engagement in antisocial activities that do not require regulated behaviour, or activities that promote under regulation.

Maturation of the frontal lobes continues through early adulthood for all adults (Romine \& Reynolds, 2005). For LCP offenders, this maturation may facilitate better cognitive impulse control ability, but yet not lead to more regulated behaviour, because the LCP offenders' antisocial lifestyles maintain them in a social environment that limits opportunities for impulse control to be modelled, practiced and positively reinforced. For 
example, in Moffitt and colleagues' 26-year follow-up results, LCP offenders had chaotic lifestyles characterised by activities that would increase contact with criminal peers (e.g., drug-trafficking), create instability in personal and family relationships, and give little opportunity for protective ventures, like employment (Moffitt, Caspi, Harrington \& Milne, 2002). As Caspi and Roberts (2001) assert, people tend to select environments that provide a comfortable fit with their own temperament. For LCP offenders who select chaotic environments, opportunities to learn the benefits of impulse control will continue to be limited or alternatively, opportunity for positive reinforcement of uncontrolled, unpredictable behaviour may be increased (Caspi \& Roberts, 2001).

Conceivably, by slightly later in adulthood, LCP offenders could have learnedperhaps from years of experiencing legal punishment from uncontrolled behaviour-that impulse control is important, thus prompting the need to practice and strengthen such skills. This assertion could explain normal decision-making and basic inhibitory abilities of offenders in the present study. Although speculative, it could also be around this time that offenders begin to notice that they are no longer obtaining the same thrills from the same antisocial activities. However, for offenders who do not learn the importance of impulse control because their environment continues to reinforce uncontrolled behaviour, or for offenders who recognise the importance of impulse control but have chronically impaired cognitive impulse control ability, cognitive impulse control weaknesses are likely to maintain risk for antisocial behaviour.

Assuming absence of severe neurological damage, it is suggested that cognitive impulse skills lie dormant in adulthood until the environment requires, motivates or positively reinforces the use of such skills. As Denney (1984) suggested, every individual 
has a certain degree of unexercised potential that can be developed as a function of optimal environmental input and training. Despite the maturation of neural mechanisms that would facilitate better impulse control in adulthood, the absence of optimal environmental conditions for LCP offenders may leave cognitive potential unexercised.

Research supports linear decreases in both cognitive and personality impulsivity with age (e.g., Romine \& Reynolds, 2005; Steinberg, Albert, Cauffman, Banich, Graham \& Woolard, 2008; Vaidya, Latzman, Markon \& Watson, 2010). However, I suggest that cognitive impulsivity may decrease relatively more than personality impulsivity for LCP offenders as a function of reinforcement experiences. Although future research is required to validate this assertion, it is hypothesised that if personal preference for risk and excitement increases risk of involvement in antisocial behaviour, and involvement in antisocial behaviour is positively reinforced, this will serve to maintain personality impulsivity via the development of positive expectancies about crime as the vehicle for providing risk and excitement (Walters, 2000, 2003). Even if the same antisocial activities weaken in their capacity to elicit thrill and excitement, offenders may simply move on to other novel antisocial activities that provide positive reinforcement and consequently, new positive expectancies will be developed in relation to different types of crime. Positive expectancies about crime thus positively reinforce personality impulsivity, and perpetuate the maintenance of antisocial behaviour (Walters, 2000, 2003).

In summary, Moffitt's LCP (1993) theory predicted that both cognitive and personality types of impulsivity contribute to problematic, antisocial behaviour early in life. However, low-level cognitive impulsivity and high VRS/VRS-SO impulsivity 
observed in this research with adult offenders highlights the possibility that cognitive and personality impulsivity follows different developmental trajectories. Whilst cognitive impulsivity may reduce by adulthood, personality impulsivity might remain relatively more stable as a function of reinforcement experiences. The capacity for cognitive mechanisms of impulse control to improve in adulthood has important implications for change in criminal risk overall, and in other crucial areas of risk including emotion regulation.

\subsubsection{The interplay between cognitive impulse control, criminal risk and emotion} regulation

Theoretically driven frameworks linking risk factors together are crucial for clinicians to use in risk assessment and clinical formulation (Ward, Polaschek \& Beech, 2005). The development of theory regarding relationships between risk factors will also inform where treatment intervention efforts should be directed first, and whether change on one risk factor might engender change on other risk factors (Polaschek, 2010b). This research found that some pre-treatment cognitive impulse control outcomes predicted post-treatment change in overall dynamic risk and anger-related outcomes. Thus, the development of theoretical frameworks for understanding how improvements in cognitive impulse control might improve other risk factors is important for informing treatment interventions.

\subsubsection{Cognitive impulse control and dynamic criminal risk}

The findings outlined in Chapter 7 showed that the cognitive impulse control abilities an offender possessed when they arrived at treatment was not associated with their pre-treatment dynamic criminal risk. However, better decision-making and better 
ability to resolve cognitive interference from dominant thoughts (i.e., inhibition), predicted how much post-treatment progress offenders made in reducing their overall dynamic criminal risk. The theoretical implications of a finding of this kind may be that those who make the most positive change in dynamic risk factors are those who have the best cognitive ability to evaluate short and long-term consequences of behaviour, and to inhibit cognitive interference from dominant thoughts before those thoughts influence behaviour.

It follows then that poorer decision-making and inhibition represent important responsivity factors that could impede treatment responses and capacity to reduce criminal risk (Andrews \& Bonta, 2006; Wong, 2000). Arguably, even for highly motivated offenders, if pro-social long-term goals are not consistently kept in mind, the benchmarks for developing and acting on avoidance goals are absent. Moreover, even if future outcomes are considered and avoidance goals are developed, inhibiting behaviour that is at odds with those goals will be difficult if offenders cannot resist the cognitive interference generated by automatic impulses to behave in ways that were appropriate in the past.

Although both decision-making and basic inhibition skills were generally intact in the present sample, it is important to bear in mind that $13 \%$ of the sample did display clinically impaired decision-making; and despite negligible clinical impairment, inhibition was still below normative averages. Therefore the questions remain as to: 1) whether current risk assessment tools can reliably identify offenders who have difficulty with these cognitive abilities, and 2) whether current cognitive-behavioural treatment 
interventions provide sufficient and appropriate opportunities to practice and consequently strengthen these important cognitive skills.

Ultimately, whilst treatment interventions can teach the value of impulse control and provide general opportunities for practicing impulse control within the treatment context, there is no guarantee that offenders who need to practice impulse control the most will actually practice; or could practice, if the basic cognitive skills needed for impulse control are impaired. Consistent with the notion of unexercised potential (Denney, 1984), and the analogy of impulse control as a muscle that requires practice to be strengthened (Muraven, Baumeister \& Tice, 1999), it is suggested that interventions specifically designed to enhance cognitive skills like decision-making and inhibition would better assist offenders to strengthen impulse control and consequently, behave in accordance with avoidance goals to reduce criminal risk. More discussion on this possibility follows in section 8.3.3.

\subsubsection{Cognitive impulse control, anger and anger control}

Moffitt's (1993) LCP theory suggested that both disinhibition and negative emotionality contribute to difficult temperament. However Moffitt's theory does not specify how inhibitory deficits and negative emotionality interact with each other to contribute to difficult temperament. It is possible that more intense negative emotion would consistently deplete the cognitive resources needed for inhibition, but it is also possible that poor inhibitory ability would exacerbate emotion: cognitive strategies to reduce the intensity of emotional experiences — such as distraction — are more difficult to engage (Denson, Moulds \& Grisham, 2012). 
The findings of this research were consistent with previous research and Moffitt's prediction that cognitive impulse control and emotion regulation are related (e.g., Denson, Moulds \& Grisham, 2012; Pessoa, 2005; Pessoa, McKenna, Gutierrez \& Ungerleider, 2002; Rusting \& Nolen-Hoeksema, 1998). The process of emotion regulation has numerous stages; from the activation of a goal to regulate the magnitude or duration of the emotional response, to controlling how emotion is expressed (Gross, Sheepes \& Urry, 2011; Gross \& Thompson, 2007; Gross, 1998). Previous research suggests that cognitive control facilitates emotion regulation at each different stage of the emotion regulation process (Clore \& Centerbar, 2004; Gross, 2013; Gross, Sheppes \& Urry, 2011; MacLeod \& Bucks, 2011; Siemer, Mauss \& Gross, 2007).

The directions of the relationships observed in this research supported the notion that cognitive control is important at the early stage of emotion generation, and at the later stage of emotion regulation (Gross \& Thompson, 2007; Gross \& Munoz, 1995). In other words, cognitive control mechanisms are necessary for decreasing the intensity of emotion, and increasing emotional control. Specifically, the findings of this research showed that cognitive impulse control was not associated with how angry offenders were, or how well they were able to control anger when they arrived at treatment. However, better pre-treatment cognitive impulse control skills-specifically inhibition and cognitive flexibility — predicted how much post-treatment progress offenders made in reducing self-report anger and increasing self-reported anger control. The direction of the relationships between cognitive impulse control and the ability to modulate angry feelings and control the expression of anger highlights important clinical implications regarding risk assessment and targets for treatment intervention. 


\subsection{Clinical implications}

\subsubsection{Risk assessment and clinical formulation}

As outlined above, the results of this research highlight the importance of identifying and differentiating cognitive, personality and non-impulsive explanations for impulsive-looking behaviour in formulations of impulsivity. Each causal mechanism has very different implications for interventions. The results of this research also highlight which psychological processes could take priority in treatment interventions to maximise benefits in other risk areas. For example, for offenders who score highly on pre-treatment assessments of anger, formulations should focus on methods for strengthening inhibition and cognitive flexibility, rather than focusing on anger-specific mechanisms of change. In contrast, for offenders with intact cognitive impulse control, focusing on anger management would be an optimal treatment strategy: these offenders would be more likely to posses the cognitive capacity to benefit from such treatment (Edmondson \& Conger, 1996).

Without strengthening inhibition and cognitive flexibility_especially for offenders with clinical impairment in these areas - it is less likely treatment progress in anger reduction and control will occur. Specifically, the effectiveness of educating offenders about emotion management strategies (e.g., removing oneself from an angerprovoking situation to cool off), and their ability to practice those strategies will be limited if the requisite cognitive weaknesses are not addressed first. Furthermore, evaluating an offender's treatment progress on their ability to develop better emotion regulation may not provide a fair representation of progress if an integral part of the problem was not addressed in treatment. 
Because clinical impairment was most pronounced on tasks requiring cognitive flexibility, and some cognitive flexibility outcomes did not significantly improve following treatment in this study, there appears to be a need for developing this important skill more effectively. Because cognitive-behavioural treatment intervenes at the level of attitudes and behaviour, not neurocognitive functioning, it is hardly surprising that such interventions may not be able to efficiently strengthen skills like cognitive flexibility (Brewin, 1996; Blackurn, 1993). As suggested previously, the implementation of cognitive training paradigms that directly aim to strengthen cognitive impulse control skills represent a promising intervention option worthy of further attention. For cognitive training programmes to be effective, important questions require further investigation to develop theory regarding mechanisms of change: which specific cognitive processes should be the focus of interventions to strengthen cognitive impulse control?

\subsubsection{Specifying mechanisms of change for cognitive interventions}

For impulse control to be successful, a number of general cognitive abilities must function normally. Currently, it is unclear whether cognitive training interventions should be directed toward impulse control-specific processes, or more general abilities (Wiers, Gladwin, Hofmann, Slemink \& Ridderinkhof, 2013). Previous neuropsychological research has used mathematical modelling to establish common (i.e., unifying) and unique (i.e., diversifying) functions underlying neuropsychological task performance (Teuber, 1972). Using such techniques, Miyake and Friedman (2012) found that outcomes from tasks representing three broad domains of executive function—updating, set shifting and basic inhibition — could be explained by one common, unifying function. The authors proposed that the common function is the "ability to actively maintain task 
goals and goal-related information and use this information to effectively bias lower-level processing" (Miyake \& Friedman, 2012, p.11). The authors also proposed that this common function accounts for the ability of outcomes from tests of updating, set shifting and inhibition to explain clinically important behaviours. When Miyake and Friedman accounted for the common function, the unique explanatory power of basic inhibition disappeared, whilst unique variance remained for updating and set shifting. Put another way, basic inhibition was explained more by the common function than by unique, inhibition-specific variance. In contrast, updating and set shifting were found to be distinctive functions capturing more than the ability to maintain and apply goal-related information. Therefore, Miyake and Friedman's findings suggest that strengthening the common function would generally improve basic inhibition, whilst more specific training might be necessary to strengthen set shifting (i.e., cognitive flexibility), because this skill is relatively more complex. Establishing which cognitive abilities might support common and unique functions represents a promising place to begin theorising about possible mechanisms of change for cognitive impulse control interventions.

It is likely that keeping goal-related information online, and shifting attention between goal-related information and the task at hand, would rely on abilities like selective attention and working memory (Diamond, 2013; Engle, 2002; Hinson, Jameson \& Whitney, 2002; Kane \& Engle, 2002; Norman \& Shallice, 1986; Posner \& Rothbart, 2000). Selective attention is the ability to selectively process certain information in the environment whilst ignoring other information; working memory relates to the ability to retain task-relevant information in an accessible state over time (Fougnie, 2008). Like attention, working memory is typically impaired in antisocial populations relative to 
normative comparisons (e.g., Herrero, Escorial \& Colom, 2010; Ogilvie et al., 2011). Although some relationships remain a source of debate, previous research shows that attention and working memory are associated with performance on some of the assessment tasks used in the present study (e.g., Hinson, Jameson \& Whitney, 2003; Jameson, Hinson \& Whitney, 2004; Losel \& Shmucker, 2004; Sanchez-Cubillo et al., 2009). Therefore, targeting general skills like attention and working memory could be an advantageous, all-inclusive approach for cognitive impulse control interventions.

\subsubsection{Empirical support for cognitive interventions}

Small pre-post treatment changes in cognitive impulse control were observed in this research, but the mechanisms of change are still unclear. Whilst more work is needed to investigate mechanisms of change, the relationship between cognitive impulse control and reductions in overall criminal risk suggests that there may be value in implementing cognitive training interventions as an adjunct therapy alongside the existing CBT treatment framework delivered in New Zealand STURPs. Because cognitive impulse control mechanisms were also important facilitators of change, these abilities represent important respsonsivity factors. Thus, the implementation of cognitive training interventions before treatment may also be of value for maximising treatment responsivity.

A number of studies investigating outcomes from training interventions for attention and working memory support training-related improvements on trained tasks (see Weirs et al., 2013 for a comprehensive review). However, generalisation to untrained tasks and, most importantly, to real-world behaviour is still debated (Karbach \& Schubert, 2013; Kray, Karbach, Haenig \& Freitag, 2012). For example, one recent study 
found that working memory retraining alone was associated with reduced drinking for problem drinkers who had strong underlying positive evaluations of alcohol (Houben, Wiers \& Jansen, 2011). In contrast, another study found that working memory training for stimulant-abusers led to greater willingness to wait for reward, but improvements in other cognitive processes and actual behaviour were not observed (Bickel, Yi, Landes, Hill \& Baxter, 2011).

Shipstead, Hicks and Engle (2012) suggest that poor skill generalisation is due to underdeveloped theories about why and how certain cognitive functions are impaired in specific clinical groups, and therefore how cognitive retraining interventions should be expected to improve specific functions. There are a number of questions that require further investigation to establish how cognitive interventions are expected to improve impulse control with offenders. For example, training improvements demonstrated to date might be explained by people learning compensatory strategies or other ways of completing a training task more effectively that do not rely on the impaired ability (Burgess \& Robertson, 2002). Logie (2012) suggested that it is important to consider the possibility that improvement on a particular training task could simply reflect better ability to deploy a broader range of cognitive skills for completing the same task. Such a suggestion is consistent with Chaytor's (2004) findings, which showed that environmental cognitive demands and compensatory strategy use accounted for unique variance in everyday executive ability over and above scores on traditional tests.

Accordingly, it is important to clarify whether cognitive interventions should be applied as direct remediation strategies to ameliorate sub-optimal cognitive impulse control functions, or at the level of teaching compensatory strategies that do not rely on 
sub-optimal cognitive impulse control functions (Cicerone, Levin, Malec, Stuss \& Whyte, 2006). For example, a compensatory strategy for offenders who find it difficult to maintain and use goal-related information would be the provision of feedback systems during treatment that provide reminders about goals or provide simple interruptions requiring articulation of the goal being pursued (Burgess \& Robertson, 2002; Linehan, 1993). Exploring whether alternative strategies of impulse control are related in the same way to the outcomes explored in this research, represents a focus for future empirical investigations.

\subsubsection{Summary of clinical implications}

Together, the results of this research highlight the importance of basing clinical formulations of impulsivity on theoretically and empirically supported causal relationships. Commonly used criminal risk instruments seem to confound cognitive, personality and non-impulsive explanations for impulsive-looking behaviour in their rating criteria. Furthermore, their reliance on naturalistic behaviour makes it difficult to make reliable inferences about underlying causality even if those inferences are theoretically driven. Reliable assessment of cognitive impulse control via cognitive tasks is important not only for understanding strengths or weaknesses in impulse control per se, but also for understanding an offender's capacity for risk reduction and treatment progress in other crucial areas that rely on cognitive impulse control, such as emotion regulation. 


\subsection{Limitations and key areas for future investigation}

\subsubsection{Inclusion of personality impulsivity and other measures}

The present study was specifically designed to explore impulsivity using the cognitive approach because relatively less attention has been paid to this approach with offenders. What emerged from the findings were indications that cognitive impulsivity is something quite different from the phenomena that clinicians identify as 'impulsive' in criminal risk assessments; namely, personality impulsivity or non-impulsivity (i.e., deliberate, considered approach to antisocial options). One limitation of the present study is that the proposed differentiation between cognitive and personality impulsivity is currently based on speculation: personality impulsivity was not directly measured.

To further understand how clinically meaningful the differentiation between cognitive and personality types of impulsivity is, future studies should include personality assessments of impulsivity, such as Eysenck's $I_{7}$ questionnaire (Eysenck, Pearson, Easting \& Allsopp, 1985). Future studies could also investigate more fully what clinicians are capturing in VRS/VRS-SO impulsivity ratings. Specifically, VRS/VRS-SO evidence recording sheets could be coded to investigate whether clinicians are typically using behavioural evidence that reflects cognitive, personality or non-impulsive explanations of impulsivity. Incorporating personality assessments would allow comparisons between the utility of personality and cognitive impulsivity outcomes in predicting criminal risk outcomes. It is particularly important in understanding the ecological validity of the cognitive approach, and its contribution relative to the personality approach, that we establish if there are differential predictive relationships 
between cognitive and personality impulsivity and recidivism. Furthermore, it would be of value to investigate if, and therefore how, cognition and personality interact: does preference for an unpredictable, spontaneous lifestyle also mean that cognitive impulse control is weak because it is never practiced? Are personality and cognitive impulsivity dissociable treatment needs?

Another promising avenue for future investigation is to assess general cognitive abilities like sustained attention and working memory to establish overlap between these functions and cognitive impulse control. Again, without assessing such abilities in the present study, I am limited to speculation about the importance of those skills as potential mechanisms of treatment change to improve impulse control. The development of theory regarding mechanisms of change is particularly important to remedy existing limitations of cognitive interventions that suffer from underdeveloped theory in this regard (Shipstead, Hicks \& Engle, 2012).

Lastly, predictive relationships examined in the present study were limited to total dynamic risk on the VRS and anger outcomes on the STAXI. Total dynamic scores on the VRS represent the sum of 20 different risk factors. Thus, total dynamic scores provided a general indication of shared variance between cognitive impulse control and dynamic sources of risk, but they could not inform which specific mechanisms were underlying this relationship. Whilst it is clear from the findings in Chapter 7, that cognitive impulse control accounts for change in emotion regulation, it might be even more important for change on other risk factors that were not explored individually in this study. Exploring relationships between cognitive impulse control and outcomes from other psychometric measures administered during the STURPs' pre-treatment assessment 
is one possibility for adding to the sparse theoretical understanding of the interplay between different risk factors (Ward, Polaschek \& Beech, 2005).

\subsubsection{Larger sample sizes for increasing statistical power in existing analyses and recidivism analyses}

The present study was not able to explore whether cognitive impulse control outcomes were predictive of recidivism. The small sample size overall meant that an even smaller proportion of the sample had been released since the post-treatment assessment and had spent enough time in the community to make recidivism analyses feasible. Future research will benefit from larger sample sizes to ensure that more offenders are eligible for recidivism analyses. Larger sample sizes also provide the statistical power needed for more sophisticated mathematical modelling (i.e., structural equation modelling) to explore mediation and moderation relationships between predictors and outcome (Kenny \& McCoach, 2003). The cross-lag correlations provided an intermediary between standard correlations and structural equation modelling (Kenny, 1975). However, establishing mediation and moderation relationships within one statistical model would provide a more sophisticated framework for understanding the interplay between impulsivity, other risk factors and risk-relevant outcomes.

\subsubsection{Manipulation of assessment context and experimental stimuli}

It is not always easy to bridge the gap between experimental research and real life symptoms (Stuss \& Knight, 2002). It is possible that the normality of some cognitive impulse control functions could be attributed to the assessment context itself or to innocuous experimental stimuli. The highly structured nature of the tasks themselves and the quiet, calm assessment context could have facilitated better impulse control 
(Salthouse, Atkinson \& Berish, 2003). Although the ecological validity of cognitive assessment tasks was supported by the predictive links to the VRS and STAXI, conducting assessments in more socially complex, unsettled testing environments could further increase ecological validity. Given more pronounced difficulty with cognitive flexibility in this sample, less structured testing environments with more distractions could be especially helpful in obtaining a more realistic view of impulse control for highrisk offenders who typically live chaotic lifestyles (e.g., Sung \& Richter, 2006).

In addition to adding distraction to tax cognitive flexibility, other changes to the assessment context could be achieved experimentally by state-based manipulation of factors known to affect impulse control such as physiological arousal (e.g., Eysenck, 1993; Revelle, Anderson \& Humphreys, 1987), positive mood (e.g., Bohner, Chaiken \& Hunyadi, 1994; De Vries, Holland \& Witteman, 2008), negative emotion (e.g., Knapp \& Clark, 1991; Tice, Bratslavsky \& Baumeister, 2001), and cognitive resource depletion (e.g., Muraven \& Baumeister, 2000; Muraven, Tice \& Baumeister, 1999). Although stable, trait-like reflective system impairments were not observed in this study, comparisons of cognitive impulse control under standard assessments conditions and following state-based manipulations would assist theory development regarding the etiological relevance of state-based reflective system impairments.

Using tasks with less abstract decontextualised stimuli than those used in the present study could increase the ecological validity of cognitive assessments in offender samples (Rodriguez-Fornells, Lorenzo-Sera, \& Andres-Pueyo, 2002; Zelazo \& Muller, 2002). Tailoring assessment tasks by using etiologically relevant stimuli may further the development of single factor theories of the relationship between cognitive impulse 
control and antisocial behaviour (Ward \& Beech, 2006). A more etiologically valid evaluation of relative imbalance between reflective and impulsive systems might be achieved if assessment tasks were tailored to each individual's impulsive system: experimental stimuli that activate reflective-impulsive conflict for one offender, may not activate conflict to the same degree for another offender (Hoffman \& Friese, 2008). The importance of tailoring tasks to account for variation in impulse strength is highlighted by the central tenets of Bechara's (2005) neurocognitive framework: impulsive behaviour can be caused by weaknesses in the reflective system or hyperactivity in the impulsive system. Empirically, cognitive studies also show that the prediction of behaviour depends on accounting for individual variation in both impulse strength and reflective abilities (see Friese, Wanke \& Hofmann, 2009).

Tailoring tasks to align with the types of decision-making dilemmas high-risk offenders might realistically face is one possibility. For example, the gambling task in this study used money as reinforcement, which is a universally desired commodity. Because most people desire money, its use might reduce inter-individual variation in impulse strength and therefore variation in how hard the reflective system must work to override an impulsive response. Providing conflict between an antisocial highreward/high-loss option (e.g., selling drugs), and a pro-social, low-reward/low-loss option (e.g., finding a job), might be one possibility for creating greater variation in impulse strength, and therefore a more ecologically valid test of reflective strength.

Clinical studies support greater ecological validity when stimuli are tailored to specific characteristics of different clinical groups. For example, alcohol-dependent subjects experience greater cognitive interference from alcohol-related words compared 
to neutral words on tests similar to the Colour-Word Interference test used in this research (Bruce \& Jones, 2004; Cox, Fadardi \& Pothos, 2006; Sharma, Albery \& Cook, 2001; Stormark, Laberg, Nordby \& Hugdahl, 2001). Similarly, individuals with Generalised Social Anxiety Disorder experience greater cognitive interference for threatrelated words compared to neutral words, and to emotional faces compared to neutral faces (e.g., Bradley, Mogg, \& Millar, 2000; Mogg \& Bradley, 2005). Tailoring the experimental context to subjects' personality traits can also affect cognitive impulse control: Wilkowski and Robinson (2007) found that in a neutral experimental context, low trait anger subjects were generally no better at response inhibition than high trait anger subjects. However, when the experimental context determined that hostile thoughts were activated first, low trait anger subjects outperformed high trait anger subjects in response inhibition. In explanation of these findings, it was suggested that low trait anger subjects are more likely to recruit cognitive control mechanisms in a hostile context to lessen the influence of hostile thoughts on subsequent emotion and behaviour (Wilkowski \& Robinson, 2008). Consistent with this explanation, social response reversal theory (Blair, 2003) predicts that for subjects low in trait anger, angry expressions from others act as a social indicator to inhibit the behaviour triggering anger. Conversely, for individuals high in trait anger, angry expressions serve to prime motivation for revenge and response execution, not response inhibition (Wilkowski \& Robinson, 2010). In support of social response reversal theory, Wilkowski (2012) found that when angry faces were used as the stop signal in the stop signal task, or as a prime before a stop stimulus, low trait anger subjects inhibited responses faster than they did when neutral faces were presented as the stop signal or the prime. In contrast, high trait anger subjects inhibited 
responses slower when the angry face was used, relative to inhibition speed when neutral faces were used. Impaired response inhibition to angry but not happy faces is also associated with higher trait aggression, supporting the influence of emotion-based influences on anger control failure as well (Denny \& Siemer, 2012).

\subsubsection{Inclusion of non-treated, matched control group}

In interpreting treatment effects, it is highly desirable to include a control group that does not receive the intervention, which was not possible in the present study and therefore represents a major limitation (Gathercole, Dunning \& Holmes, 2012). The optimal design for future investigations would be to recruit a non-treated, incarcerated control group matched on factors known to relate to recidivism, as well as factors known to relate to neuropsychological performance. Comparisons between treated and untreated controls are not only important for understanding the influence of treatment on cognitive impulse control outcomes, but also for understanding the reliability of cognitive assessment tasks over multiple administrations; a crucial aspect of evaluating treatment change. Some of the cognitive tasks used in the present study, such as the Iowa Gambling Task, did not have adequate sources of test-retest reliability data (Buelow \& Suhr, 2009). If positive change in cognitive impulse control was observed for treated but not untreated controls, this would provide stronger support for the positive effect of treatment than statistical comparison of pre and post-treatment outcomes as in the present study. If positive change in cognitive impulse control was observed for both treated and untreated offenders, this could indicate that improvements were more likely due to extraneous factors, such as practice effects on assessment tasks (Beglinger et al., 2005). 


\subsubsection{Comparisons of cognitive impulse control between offender sub-groups}

As outlined in Chapter 2, a number of individual-level factors can influence whether cognitive impulse control is impaired; or at least, relatively poorer in some offender sub-groups than others. One important factor is whether offenders are on the life-course-persistent (LCP) or adolescence-limited (AL) path (Moffitt, 1993). Offenders in this study were recruited purely on the basis of high static risk scores and placement in a treatment programme. Because the factors that determine high static risk parallel the features of Moffitt's (1993) LCP typology (e.g., extensive offending history, serious offending history, early age of first conviction: Bakker, O’Malley, \& Riley, 1998), specific hypotheses could be tested about cognitive impulse control based on the assumption that the majority of high-risk offenders in the present study were likely to be on the LCP path (Wilson, 2004). However, the LCP/AL distinction was not tested in this study. Although there were indications that the majority of the sample fit the LCP typology (e.g., high mean static item scores on VRS items indicating violence across the lifespan and high degrees of family dysfunction and instability), more data from file information (e,g,, psychological reports, VRS evidence recording sheets), was needed to verify the time line of each offender's involvement in antisocial behaviour and to provide a direct test of Moffitt's theory.

However, beyond the LCP/AL distinction, another important distinction to assist theory development would be to compare cognitive impulse control ability between offenders with, and without histories of brain insult. Elevated rates of brain injury are common in incarcerated, particularly violent samples, compared to the general population (Hancock, Tapscott \& Hoaken, 2010). Consistent with estimations in other parts of the 
world, a 1998 study of New Zealand prisoners in the Waikato region showed that $86.4 \%$ of offenders had sustained a traumatic brain injury at some time in their lives (Barnfield \& Leatham, 1998). In a relatively more recent New Zealand study, Crump (2005) found that $86 \%$ of violent offenders and $81 \%$ of non-violent offenders received a head injury prior to their first conviction. It is unclear whether offenders who displayed clinically impaired performance in this study also had a history of brain injury.

Establishing histories of brain injury is important for delineating whether braininjured offenders are more impaired and require different types of intervention for impaired impulse control than offenders without a history of brain injury. Encouragingly, previous studies have illustrated training-related performance improvements for people who have experienced traumatic brain injury (e.g., Sacco et al., 2011). However, it may be that offenders with brain injury benefit relatively less from cognitive interventions to strengthen cognitive impulse control than offenders without brain injury; this could have important implications for limited treatment progress on other outcomes, like emotion regulation.

Two other individual-level factors that could affect cognitive impulse control are a history of severe substance use (e.g., Bechara, 2007), and historical or current Attention Deficit/Hyperactivity Disorder (ADHD) symptomatology. Barry and Petry (2008) found that lifetime history of substance use disorder reduced advantageous decision-making performance on the Iowa Gambling Task regardless of current substance use, suggesting that substance use can affect decision-making beyond remission. A marked number of adult inmates suffer from ADHD symptoms as children and continue to have these traits in adulthood (Eyestone \& Howell, 1994). Furthermore, some neural information 
processing deficits found in ADHD are even more pronounced in offenders with ADHD (Meier, Perrig \& Koenig, 2012). Thus, differentiating adult offenders with ADHD symptoms from those without represents an important research endeavour to establish whether unique patterns of cognitive impulse control impairment emerge as a function of these specific clinical characteristics.

\subsection{Summary}

The present study took a preliminary step toward investigating cognitive mechanisms of impulse control in a high-risk adult offender sample. Overall, although the study design had a number of significant limitations, a relatively hopeful picture emerged. Despite predictions of significant impairment, high-risk offenders were not especially impaired in their ability to carry out the basic cognitive functions that support impulse control. Furthermore, the provision of a structured environment that necessitated the practice of cognitive impulse control went some way in improving those functions. Although there is a need for a great deal of research to follow this preliminary investigation, the results tentatively suggest that implementing interventions specifically designed to facilitate and strengthen cognitive impulsive control could have direct bearings on other risk factors. Improving cognitive impulse control functions may serve to improve an offenders' ability to effectively process treatment material. Ultimately, such improvements could foster the development of cognitive skills necessary to plan for life after prison, and successfully reintegrate into the community. 


\section{References}

Aarts, H., \& Dijksterhuis, A. (2000). Habits as knowledge structures: Automaticity in goaldirected behavior. Journal of Personality and Social Psychology, 78, 53-63.

Aharonovich, E., Nunes, E.V., \& Hasin, D. (2003). Cognitive impairment, retention and abstinence among cocaine abusers in cognitive-behavioral treatment. Drug and Alcohol Dependence, 71, 207-211.

Alderman, N., Fry, R.K., \& Youngson, H.A. (1995). Improvement of self-monitoring skills, reduction of behaviour disturbance and the dysexecutive syndrome: Comparison of response cost and a new programme of self-monitoring training. Neuropsychological Rehabilitation: An International Journal, 5(3), 193-221.

American Psychiatric Association. (2000). Diagnostic and statistical manual of mental disorders ( $4^{\text {th }}$ ed., text rev.). Washington DC: Author.

Amieva, H., Phillips, L., \& Della Salla, S. (2003). Behavioral dysexecutive symptoms in normal aging. Brain and Cognition, 53, 129-132.

Anderson, C.A. \& Bushman, B.J. (2002). Human aggression. Annual Review of Psychology, $53,27-51$.

Andrews, D.A., \& Bonta, J. (2006). The Psychology of Criminal Conduct (4 ${ }^{\text {th }}$ ed.), New Providence, NJ: Matthew Bender \& Company, Inc.

Andrews, D.A., Bonta, J., \& Wormith, S. (2004). The Level of Service/Case Management Inventory (LS/CMI). Toronto, Canada: Multi-Health Systems.

Andrews, D.A., Bonta, J., \& Wormith, J.S. (2006). The recent past and near future of risk and/or need assessment. Crime and Delinquency, 52(1), 7-27.

Arbuckle, J. L. (2010). IBM SPSS Amos 19 User's Guide. Amos Development Corporation. 
Babinski, L.M., Hartsough, C.S., \& Lambert, N.M. (1999). Childhood conduct problems, hyperactivity-impulsivity, and inattention as predictors of adult criminal activity. Journal of Child Psychology \& Psychiatry, 40(3), 347-355.

Bakker, L., O’Malley, J., \& Riley, D. (1998). Storm Warning: Statistical Models for Predicting Violence. Christchurch: Psychological Service, Department of Corrections. Bandura, A. (1986). Social Foundations of Thought and Action: A Social Cognitive Theory. Englewood Cliffs, NJ: Prentice Hall.

Barkataki, I., Kumari, V., Das, M., Sumich, A., Taylor, P., \& Sharma, T. (2008). Neural correlates of deficient response inhibition in mentally disordered violent individuals. Behavioral Sciences and the Law, 26, 51-64.

Barkley, R. A. (1991). The ecological validity of laboratory and analogue assessment methods of ADHD symptoms. Journal of Abnormal Child Psychology, 19(2), 149-178.

Barkley, R.A., Fischer, M., Smallish, L., \& Fletcher, K. (2002). Persistence of attention deficit hyperactivity disorder into young adulthood as a function of reporting source and definition of disorder. Journal of Abnormal Psychology, 111, 279-289.

Barnfield, T. V., \& Leathem, J. M. (1998). Incidence and outcomes of traumatic brain injury and substance abuse in a New Zealand prison population. Brain Injury, 12, 455-466.

Barratt, E.S. (1985). Impulsiveness substrates: Arousal and information processing. In J.T. Spence \& C.E Izard (Eds.), Motivation, Emotion and Personality (pp. 137-146). New York: Elsevier.

Barratt, E.S. (1993). Impulsivity: integrating cognitive, behavioral, biological, and environmental data. In W.G. McCown, J.L. Johnson \& M.B. Shure (Eds.), The 
Impulsive Client: Theory, Research, and Treatment (pp. 39-56). Washington DC:

American Psychological Association.

Barry, D., \& Petry, N. M. (2008). Predictors of decision-making on the Iowa Gambling Task: Independent effects of lifetime history of substance use disorders and performance on the Trail Making Test. Brain and Cognition, 66, 243-252.

Bauer, L. O. (2001). Predicting relapse to alcohol and drug abuse via quantitative electroencephalography. Neuropsychopharmacology, 25, 332-340.

Baumeister, R.F. (2002). Ego depletion and self-control failure: An energy model of the self's executive function. Self and Identity, 1, 129-136.

Baumeister, R.F., \& Heatherton, T.F. (1996). Self-regulation failure: An overview. Psychological Inquiry, 7(1), 1-15.

Baumeister, R. F., Heatherton, T. F., \& Tice, D. M. (1994). Losing control: How and Why People Fail at Self-regulation. San Diego, CA: Academic Press.

Bechara, A. (2005). Decision making, impulse control and loss of willpower to resist drugs: a neurocognitive perspective. Nature Neuroscience, 8(11), 1458-1463.

Bechara, A. (2007). Iowa Gambling Task Professional Manual. Lutz: Psychological Assessment Resources.

Bechara, A., \& Damasio, H. (2002). Decision-making and addiction (part I): impaired activation of somatic states in substance dependent individuals when pondering decisions with negative future consequences. Neuropsychologia, 40(10), 1675-1689.

Bechara, A., Damasio, H., \& Damasio, A. R. (2000). Emotion, decision-making and the orbitofrontal cortex. Cerebral Cortex, 10(3), 295-307. 
Bechara, A., Damasio. A.R., Damasio, H., \& Anderson, S.W. (1994). Insensitivity to future consequences following damage to human prefrontal cortex. Cognition, 50, 7-15.

Bechara, A., Damasio, H., Damasio, A.R., \& Lee, G. P. (1999). Different contributions of the human amygdala and ventromedial prefrontal cortex to decision-making. The Journal of Neuroscience, 19(13), 5473-5481.

Bechara, A., Damasio, H., Tranel, D., \& Damasio, A. R. (1997). Deciding advantageously before knowing the advantageous strategy. Science, 275(5304), 1293-1295.

Bechara, A., Dolan, S., Denburg, N., Hindes, A., Anderson, S. W., \& Nathan, P. E. (2001). Decision-making deficits, linked to a dysfunctional ventromedial prefrontal cortex, revealed in alcohol and stimulant abusers. Neuropsychologia, 39, 376-389.

Bechara, A., Dolan, S., \& Hindes, A. (2002). Decision-making and addiction (part II): myopia for the future or hypersensitivity to reward? Neuropsychologia, 40(10), 16901705.

Bechara, A., Noel, X., \& Crone, E.A. (2006). Loss of willpower: Abnormal neural mechanisms of impulse control and decision-making in addiction. In R.W. Wiers \& A.W. Stacy (Eds.), Handbook of Implicit Cognition and Addiction (pp.215-232). Thousand Oaks, CA: Sage Publications.

Bechara, A., Tranel, D., \& Damasio, H. (2000). Characterization of the decision-making deficit of patients with ventromedial prefrontal cortex lesions. Brain, 123, 2189-2202.

Bechara A., Tranel D., Damasio H., \& Damasio A. R. (1996). Failure to respond automatically to anticipated future outcomes following damage to prefrontal cortex. Cerebral Cortex, 6, 215-225. 
Beglinger, L. J., Gaydos, B., Tangphao-Daniels, O., Duff, K., Kareken, D. A., Crawford, J., Fastenau, P.S., \& Siemers, E.R. (2005). Practice effects and the use of alternate forms in serial neuropsychological testing. Archives of Clinical Neuropsychology, 20(4), $517-529$.

Bekker, E.M., Overtoom, C.C., Kenemans, J.L., Kooij, J.J., De Noord, I., Buitelaar, J.K., \& Verbaten, M.N. (2005). Stopping and changing in adults with ADHD. Psychological Medicine, 35(6), 807-816.

Bellgrove, M, A., Hester, R., \& Garavan, H. (2004). The functional neuroanatomical correlates of response variability: evidence from a response inhibition task. Neuropsychologia, 42, 1910-1926.

Bergvall, A. H., Wessely, H., Forsman, A., \& Hansen, S. (2001). A deficit in attentional setshifting of violent offenders. Psychological Medicine, 31, 1095-1105.

Bickel, W. K., Yi, R., Landes, R. D., Hill, P. F., \& Baxter, C. (2011). Remember the future: Working memory training decreases delay discounting among stimulant addicts. Biological Psychiatry, 69, 260-265.

Binder, L.M., Iverson, G.L., \& Brooks, B.L. (2009). To err is human: “Abnormal” neuropsychological scores and variability are common in healthy adults. Archives of Clinical Neuropsychology, 24, 31-46.

Bjork, J.M., Dougherty, D.M., Moeller, F.G., Harper, R.A., Scott-Gurnell, K., \& Swann, A.C. (2000). Laboratory measures of impulsivity in hospitalized adolescents with disruptive behavior disorders. Biological Psychiatry, 47(8), S149.

Blackburn, R. (1993). The Psychology of Criminal Conduct: Theory, Research and Practice. Chichester: Wiley. 
Blackburn, R. \& Fawcett, D. (1999). The Antisocial Personality Questionnaire: An Inventory for Assessing Personality Deviation in Offender Populations. European Journal of Psychological Assessment, 15, 14-24.

Blair, R. J. R. (2003). Facial expressions, their communicatory functions, and neuro-cognitive substrates. Philosophical Transactions of the Royal Society of London, B, 358, 561572.

Bohner, G., Chaiken, S., \& Hunyadi, P. (1994). The role of mood and message ambiguity in the interplay of heuristic and systematic processing. European Journal of Social Psychology, 24, 207-221.

Bornovalova, M., Daughters, S., Hernandez, G., Richards, J., \& Lejuez, C. (2005).

Differences in impulsivity and risk-taking propensity between primary users of crack cocaine and primary users of heroin in a residential substance-use program. Experimental and Clinical Psychopharmacology, 13(4), 311-318.

Bradley, B. P., Mogg, K., \& Millar, N. H. (2000). Covert and overt orienting of attention to emotional faces in anxiety. Cognition and Emotion, 14, 789-808.

Brand, M., Recknor, E.C., Grabenhorst, F., \& Bechara, A. (2007). Decisions under ambiguity and decisions under risk: Correlations with executive functions and comparisons of two different gambling tasks with implicit and explicit rules. Journal of Clinical and Experimental Neuropsychology, 29(1), 86-99.

Brehmer, Y., Westerberg, H., \& Backman, L. (2012). Working memory training in younger and older adults: Training gains, transfer and maintenance. Frontiers in Human Neuroscience, 6, 63. 
Brewin, C. R. (1996). Theoretical foundations of cognitive-behavior therapy for anxiety and depression. Annual Review of Psychology, 47, 33-57.

Brezina. T., \& Topalli, V. (2012). Criminal self-efficacy: Exploring the correlates and consequences of a "successful criminal” identity. Criminal Justice and Behavior, 39, 1042-1062.

Broomhall, L. (2005). Acquired sociopathy: A neuropsychological study of executive dysfunction in violent offenders. Psychiatry, Psychology and Law, 12, 367-387.

Bruce, G., \& Jones, B.T. (2004). A pictorial Stroop paradigm reveals an alcohol attentional bias in heavier compared to lighter social drinkers. Journal of Psychopharmacology, $18,527-33$.

Brunner, D. \& Hen, R. (1997). Insights into the neurobiology of impulsive behavior from serotonin receptor knockout mice. Annals of NewYork Academy of Science, 836, 81105.

Buck, K.K., Atkinson, T.M., \& Ryan, J.P. (2008). Evidence of practice effects in variants of the Trail Making Test during serial assessment. Journal of Clinical and Experimental Neuropsychology, 30(3), 312-318.

Buelow, M.T., \& Suhr, J.A. (2013). Personality characteristics and state mood influence individual deck selections on the Iowa Gambling Task. Personality and Individual Differences, 54, 593-597.

Buelow, M.T., \& Suhr, J.A. (2009). Construct validity of the Iowa Gambling Task. Neuropsychological Review, 19, 102-114. 
Bunge, S.A., Ochsner, K.N., Desmond, J.E., Glover, G.H., \& Gabrieli, J.D.E. (2001).

Prefrontal regions involved in keeping information in and out of mind. Brain, 124, 2074-2086.

Burgess, P., \& Robertson, I H. (2002). Principles of the rehabilitation of frontal lobe function. In D.T. Stuss \& R.T Knight (Eds.), Principles of Frontal Lobe Function (pp. 557-572). New York: Oxford University Press.

Bushman, B. J., Bonacci, A. M., Pedersen, W. C., Vasquez, E. A., \& Miller, N. (2005). Chewing on it can chew you up: Effects of rumination on triggered displaced aggression. Journal of Personality and Social Psychology, 88, 969-983.

Buss, A. H., \& Plomin, R. (1975). A Temperament Theory of Personality Development. New York: John Wiley \& Sons.

Calamia, M., Markon, K., \& Tranel, D. (2012). Scoring higher the second time around: Metaanalyses of practice effects in neuropsychological assessment. The Clinical Neuropsychologist, 26(4), 543-570.

Caprara, G. V., Barbaranelli, C., Borgogni, L., \& Perugini, M. (1993). The "big five questionnaire:" A new questionnaire to assess the five factor model. Personality and Individual Differences, 15(3), 281-288.

Carroll, A., Hemingway, F., Bower, J., Ashman, A., Houghton, S., \& Durkin, K. (2006). Impulsivity in juvenile delinquency: Differences among early-onset, late-onset and non-offenders. Journal of Youth and Adolescence, 35(4), 519-529.

Carver, C.S., \& Scheier, M.F. (1981). Attention and Self-regulation. New York: SpringerVerlag. 
Carver, C. S., \& White, T. L. (1994). Behavioral inhibition, behavioral activation, and affective responses to impending reward and punishment: The BIS/BAS scales. Journal of Personality and Social Psychology, 67, 319-333.

Caspi, A., \& Roberts, B. W. (2001). Personality development across the life course: The argument for change and continuity. Psychological Inquiry, 12, 49-66.

Chamberlain, S.R., Fineberg, N.A., Blackwell, A.D., Robbins, T.W., \& Sahakian, B.J. (2006).

Motor inhibition and cognitive flexibility in obsessive-compulsive disorder and trichotillomania. American Journal of Psychiatry, 163, 1282-1284.

Chambers, C.D., Garavan, H., \& Bellgrove, M.A. (2009). Insights into the neural basis of response inhibition from cognitive and clinical neuroscience. Neuroscience and Biobehavioral Reviews, 33(5), 631-646.

Chan, R. (2001). Dysexecutive symptoms among a non-clinical sample: A study with the use of the Dysexecutive Questionnaire. British Journal of Psychology, 92, 551-565.

Chaytor, N, S. (2004). Improving the Ecological Validity of Executive Functioning Assessment. (Unpublished doctoral dissertation). Washington State University.

Chen, M., \& Bargh, J. A. (1999). Consequences of automatic evaluation: Immediate behavioral predispositions to approach or avoid the stimulus. Personality and Social Psychology Bulletin, 25, 215-224.

Chen, C-Y., Muggleton, N.G., Juan, C-H,. Tzeng, O.J.L., \& Hung, D.L. (2008). Time pressure leads to inhibitory control deficits in impulsive violent offenders. Behavioural Brain Research, 187(2), 483-488. 
Cherek, D. R., \& Lane, S. D. (1999). Laboratory and psychometric measurements of impulsivity among violent and non-violent female parolees. Biological Psychiatry 46, 273-80.

Cherek, D.R., Moeller, G., Dougherty, D.M., \& Rhoades, H. (1997). Studies of violent and non-violent male parolees: II. Laboratory and psychometric measurements of impulsivity, Biological Psychiatry, 41, 523-529.

Cheung, A.M., Mitsis, E.M., \& Halperin, J.M. (2004). The relationship of behavioral inhibition to executive functions in young adults. Journal of Clinical and Experimental Neuropsychology, 26, 393-404.

Cicerone, K, D. (2002). Remediation of working attention in mild traumatic brain injury. Brain Injury, 16(3), 185-195.

Cicerone, K.D., \& Giacino, J.T. (2002). Remediation of executive function deficits after traumatic brain injury. Neurorehabilitation, 2, 78-83.

Cicerone, K.D., Levin, H., Malec, J., Stuss, D., \& Whyte, J. (2006). Cognitive rehabilitation interventions for executive function: moving from bench to bedside in patients with traumatic brain injury. Journal of Cognitive Neuroscience, 18(7), 1212-1222.

Cloninger, C. R., Svrakic, D. M., \& Przybeck, T. R. (1993). A psychobiological model of temperament and character. Archives of General Psychiatry, 50, 975-990.

Clore, G.L., \& Centerbar, D.B. (2004). Analyzing anger: How to make people mad. Emotion, 4, 139-144.

Cochran, W., \& Tesser, A. (1996). The "what the hell effect": Some effects of goal proximity and goal framing on performance. In L. Martin, \& A. Tessler (Eds.), Striving and 
Feeling: Interaction among Goals, Affect, and Self-Regulation (pp. 99-120). Hillsdale, NJ: Lawrence Erbaum Associates.

Cohen J. (1988). Statistical Power Analysis for the Behavioral Sciences (2nd ed.). Hillsdale, NJ: Lawrence Erlbaum.

Colder, C. R., \& O’Connor, R. M. (2004). Gray's reinforcement sensitivity model and child psychopathology: Laboratory and questionnaire assessment of the BAS and BIS. Journal of Abnormal Child Psychology, 32, 435-451.

Colder, C.R., \& Stice, E. (1998). A longitudinal study of the interactive effects of impulsivity and anger on adolescent problem behavior. Journal of Youth and Adolescence, 27(3), $255-274$.

Conners, C.K. (2002). Conners' Continuous Performance Test II. Multi-health systems.

Costa, P. T. Jr, \& McCrae, R. R. (1992). Revised NEO Personality Inventory Manual. Odessa, FL: Psychological Assessment Resources.

Coull, J.T. (1998). Neural correlates of attention and arousal: insights from electrophysiology, functional neuroimaging and psychopharmacology. Progress in Neurobiology, 55, $343-361$.

Cox, W.M., Fadardi, J.S., \& Pothos, E.M. (2006). The addiction stroop test: Theoretical considerations and procedural recommendations. Psychological Bulletin, 132, 443-76.

Crawford, J. R., Sutherland, D., \& Garthwaite, P. H. (2008). On the reliability and standard errors of measurement of contrast measures from the D-KEFS. Journal of the International Neuropsychological Society, 14, 1069-1073.

Crean, J. P., de Wit, H., \& Richards, J. B. (2000). Reward discounting as a measure of impulsive behavior in a psychiatric outpatient population. Experimental and Clinical 
Psychopharmacology, 8, 155-162.

Crump, S. A. (2005). The Neglected Offender: Exploring the Role of Executive Dysfunction in Violent Offending. (Unpublished doctoral thesis). University of Auckland, New Zealand.

Cyders, M.A., \& Coskunpinar, A. (2012). The relationship between self-report and lab conceptualizations of impulsivity. Journal of Research in Personality, 46, 121-124.

Damasio, A. R. (1996). The somatic marker hypothesis and the possible functions of the prefrontal cortex. Philosophical Transactions of the Royal Society B: Biological Sciences, 351(1346), 1413-1420.

Davidson, M.C., Amso, D., Anderson, L.C., Diamond, A. (2006). Development of cognitive control and executive functions from 4-13 years: evidence from manipulations of memory, inhibition and task switching. Neuropsychologia, 44, 2037-2078.

Delis, D.C., Kaplan, E., \& Kramer, J.H. (2001). The Delis-Kaplan Executive Function System: Examiner's Manual. San Antonio: The Psychological Corporation.

Delisi, M., \& Vaughn, M.G. (2011). The importance of neuropscyhological deficits relating to self-control and temperament to the prevention of serious antisocial behaviour. International Journal of Child, Youth and Family Studies, 1 \& 2, 12-35.

Denny, N.W. (1984). A model of cognitive development across the life span. Developemtnal Review, 4(2), 171-191.

Denny, K., \& Siemer, M. (2012). Trait aggression is related to anger-modulated deficits in response inhibition. Journal of Research in Personality, 46, 450-455.

Denson, T. (2013). The multiple systems model of angry rumination. Personality and Social Psychology Review, 17(2), 103-123. 
Denson, T. F., Moulds, M. L., \& Grisham, J. R. (2012). The effects of rumination, reappraisal, and distraction on anger experience. Behavior Therapy, 43, 355-364.

Denson, T. F., Pedersen, W. C., Friese, M, Hahm, A., \& Roberts, L. (2011). Understanding impulsive aggression: Angry rumination and reduced self-control capacity are mechanisms underlying the provocation-aggression relationship. Personality and Social Psychology Bulletin, 27, 850-862.

De Vries, M., Holland, R. W., \& Witteman, C. L. M. (2008). In the winning mood: Affect in the Iowa gambling task. Judgment and Decision Making, 3, 42-50.

Diamond, A. (2013). Executive functions. Annual Review of Psychology, 64, 135-168.

Dickman, S.J. (1990). Functional and dysfunctional impulsivity: personality and cognitive correlates. Journal of Personality and Social Psychology, 58(1), 95-102.

Dinn, W., \& Harris, C.L. (2000). Neurocognitive function in antisocial personality disorder. Psychiatry Research, 97, 173-190.

Dolan, M. (2012). The neuropsychology of prefrontal function in antisocial personality disordered offenders with varying degrees of psychopathy. Psychological Medicine, $42,1715-1725$

Dolan, M., \& Park, I. (2002). The neuropsychology of antisocial personality disorder. Psychological Medicine, 32(3), 417-427.

Dolan, M. \& Fullam, R. (2004). Behavioural and psychometric measures of impulsivity in a personality disordered population. The Journal of Forensic Psychiatry \& Psychology, $15,426-450$.

Dougherty, D.M. (1999). IMT/DMT Immediate Memory Task and Delayed Memory Task: A research tool for studying attention and memory processes (version 1.3). Houston, 
Texas: Neurobehavioral Research Laboratory and Clinic, University of Texas Health Science Center.

Dougherty, D.M., Bjork, J.M., Harper, R.A., Marsh, D.M., Moeller, F.G., Mathias, C.W., \& Swann, A.C. (2003). Behavioral impulsivity paradigms: A comparison in hospitalized adolescents with disruptive behavior disorders. Journal of Child Psychology and Psychiatry, 44, 1145-1157.

Dougherty, D.M., Bjork, J.M., Huckabee, H.C.G., Moeller, F.G., \& Swann, A.C (1999). Laboratory measures of aggression and impulsivity in women with borderline personality disorder. Psychiatry Research, 85, 315-326.

Dougherty, D.M., Bjork, J.M., Marsh, D.M., \& Moeller, F.G. (2000). A comparison between adults with conduct disorder and normal controls on a continuous performance test: Differences in impulsive response characterstics. The Psychological Record, 50, 203219.

Dougherty, D.M., Marsh, D.M., \& Mathias, C.W. (2002). Immediate and Delayed Memory Tasks: A computerized measure of memory, attention, and impulsivity. Behavior Research Methods, Instruments and Computers, 34, 391- 398.

Dougherty, D.M., Mathias, C.W., Marsh, D.M., \& Jagar, A.A. (2005). Laboratory behavioral measures of impulsivity. Behavior Research Methods 37, 82-90.

Dougherty, D.M., Mathias, C.W., Marsh-Richard, D.M., Furr, R.M., Nouvion, S.O. \& Dawes, M.D. (2009). Distinctions in behavioral impulsivity: Implications for substance abuse research. Addictive Disorders and Their Treatment, 8(2), 61-73.

Downes, J.J., Roberts, A.C., Sahakian, B.J., Evenden, J.L., Morris, R.G. \& Robbins, T.W. (1989). Impaired extra-dimensional shift performance in medicated and unmedicated 
Parkinson's disease: Evidence for a specific attentional dysfunction.

Neuropsychologia, 27, 1329-1343.

Draganski, B., Gaser, C., Busch, V., Schuierer, G., Bogdahn, U., \& May, A. (2004).

Neuroplasticity: changes in grey matter induced by training. Nature, 427, 311-312.

Eaves, D., Tien, G., \& Wilson, D. (1997). In C.D. Webster \& M.A. Jackson (Eds.), Impulsivity: Theory, Assessment and Treatment (pp.409-423). New York: Guilford Press.

Echeburua, E., \& Fernandez-Montalvo, J. (2007). Male batterers with and without psychopathy: An exploratory study in Spanish prisons. International Journal Offender Therapy and Comparative Criminology, 51, 254-263.

Edmondson, C.B., \& Conger, J.C. (1996). A review of treatment efficacy for individuals with anger problems: Conceptual, assessment, and methodological issues. Clinical Psychology Review, 16, 251-275.

Eisenberg, N., Sadovsky, A., Spinrad, T.L., Fabes, R. A., Losoya, S.H., Valiente, C., Reiser, M., Cumberland, A., \& Shepard, S. A. (2005). The relations of problem behaviour status to children's negative emotionality, effortful control, and impulsivity: Concurrent relations and prediction of change. Developmental Psychology, 41(1), 193211.

Elliott, A.J., \& Thrash, T.M. (2002). Approach-avoidance motivation in personality: Approach and avoidance temperament and goals. Journal of Personality and Social Psychology, 82(5), 804-818.

Engle, R. W. (2002). Working memory capacity as executive attention. Current Directions in Psychological Science, 11(1), 19-23. 
Enticott, P.G., \& Ogloff, J. R.P. (2006). Elucidation of impulsivity. Australian Psychologist, 41(1), 3-14.

Enticott, P.G., \& Ogloff, J. R.P, Bradshaw, J.L., \& Daffern, M. (2007). Contrary to popular belief, a lack of behavioural inhibitory control may not be associated with aggression. Criminal Behaviour and Mental Health, 17(3), 179-183.

Enticott, P.G., Ogloff, J.R.P., Bradshaw, J.L., \& Fitzgerald, P.B. (2008). Cognitive inhibitory control and self-reported impulsivity among violent offenders with schizophrenia. Journal of Clinical and Experimental Neuropsychology, 30 (2), 157-162.

Epstein, J.N., Johnson, D.E., Varia , I.M., \& Conners, C.K. (2001). Neuropsychological assessment of response inhibition in adults with ADHD. Journal of Clinical and Experimental Neuropsychology, 23(3), 362-371.

Evenden, J. (1999). Impulsivity: a discussion of clinical and experimental findings. Psychopharmacology, 13(2), 180-192.

Eyestone, L., \& Howell, R. (1994). An epidemiological study of attention-deficit hyperactivity disorder and major depression in a male prison population. Bulletin of the Academy of Psychiatry and Law, 22(2), 181-193.

Eysenck, M. W. (1982). Attention and Arousal: Cognition and Performance. Berlin: SpringerVerlag.

Eysenck, S. B. G., \& Eysenck, H. J. (1977). The place of impulsiveness in a dimensional system of personality description. British Journal of Social and Clinical Psychology, $16,57-68$.

Eysenck, S.B.G., \& McGurk, B.J. (1980). Impulsiveness and venturesomeness in a detention centre population. Psychological Report, 47, 1299-1306. 
Eysenck, S. B. G., Pearson, P. R., Easting, G., \& Allsopp, J. F. (1985). Age norms for impulsiveness, venturesomeness, and empathy in adults. Personality and Individual Differences, 6, 613-619.

Fallgatter, A.J., \& Herrmann, M.J. (2001). Electrophysiological assessment of impulsive behaviour in healthy subjects. Neuropsychologia, 39(3), 328-333.

Farrington, D.P (1989). Early predictors of adolescent aggression and adult violence. Violence and Victims, 4(2), 79-100.

Farrington, D.P. (2011). Integrated Developmental and Life-course Theories of Offending: Advances in Criminological Theory (pp.73-92). New Brunswick, New Jersey: Transaction Publishers.

Field, A. (2009). Discovering Statistics Using SPSS ( ${ }^{\text {rd }}$ ed.). London, England: SAGE. Fillmore, M. T., Dixon, M. J., \& Schweizer, T. A. (2000). Alcohol affects processing of ignored stimuli in a negative priming paradigm. Journal of Studies on Alcohol, 61, $571-578$.

Fillmore, M.T., \& Rush, C.R. (2002) Impaired inhibitory control of behavior in chronic cocaine users. Drug and Alcohol Dependence, 66, 265-273.

Finn, P.R., Mazas, C.A., Justus, A.N., \& Steinmetz, J. (2002). Early-onset alcoholism with conduct disorder: Go/NoGo learning deficits, working memory capacity, and personality. Alcoholism, Clinical and Experimental Research, 26, 186-206.

Fischer, A. H., \& Roseman, I.J. (2007). Beat them or ban them: The characteristics and social functions of anger and contempt. Journal of Personality and Social Psychology, 93, $103-115$. 
Fishbein, D.H., Hyde, C., Eldreth, D., Paschall, M.J., Hubal, R., Das, A., Tarter, R., Ialongo, N., Hubbard, S., \& Yung, B. (2006). Neurocognitive skills moderate urban male adolescents' responses to preventive intervention materials. Drug and Alcohol Dependence, $82,47-60$.

Fishbein, D., Sheppard, M., Hyde, C., Hubal, R., Newlin, D., Serin, R., Chrousos, G., \& Alesci, S. (2009). Deficits in behavioural inhibition predict treatment engagement in prison inmates, Law and Human Behaviour, 33, 419-435.

Fougnie, D. (2008). The relationship between attention and working memory. In N.B Johansen (Ed.), New Research on Short-term Memory (pp. 1-45). Nova Science Publishers, Inc.

Fowles, D. C. (1980). The three-arousal model: Implications of Gray's two-factor learning theory for heart rate, electrodermal activity, and psychopathy. Psychophysiology, 17, 87-104.

Friese, M., Wanke, M., \& Hofmann, W. (2009). In J.P. Forgas, R.F. Baumeister, \& D.M. Tice. Psychology of Self-Regulation: Cognitive, Affective, and Motivational Processes. (pp. 53-72). New York: Psychology Press.

Gage, F.H. (2002) Neurogenesis in the adult brain. Journal of Neuroscience, 22(3), 612-613. Gagne, J.R., \& Goldsmith, H. (2011). A longitudinal analysis of anger and inhibitory control in twins from 12 to 36 months of age. Developmental Science, 14(1), 112-124.

Gardner, M., \& Steinberg, L. (2005). Peer influence on risk taking, risk preference, and risky decision making in adolescence and adulthood: an experimental study. Developmental Psychology, 41(4), 625-635. 
Garon, N., Bryson, S.E., \& Smith, I.M. (2008). Executive function in preschoolers: a review using an integrative framework. Psychological Bulletin, 134, 31-60.

Gathercole, S.E., Dunning, D.L. \& Holmes, J. (2012). Cogmed training: Let's be realistic about intervention research. Journal of Applied Research in Memory and Cognition 1 (3), 201-203.

Giancola, P. R. (2000). Executive functioning: A conceptual framework for alcohol- related aggression. Experimental and Clinical Psychopharmacology, 8, 576-597.

Gibson, C. L. \& Tibbetts, S.G. (2000). A biosocial interaction in predicting early onset of offending. Psychological Reports, 86, 509-18.

Glaser, D. (2000). Child abuse and neglect and the brain: A review. Journal of Child Psychology and Psychiatry, 41(1), 97-116.

Glicksohn, J., Leshem, R., \& Aharoni, R. (2006). Impulsivity and time estimation: Casting a net to catch a fish. Personality and Individual Differences, 40, 261-271.

Glynn, L. M., Christenfeld, N., \& Gerin, W. (2002). The role of rumination in recovery from reactivity: Cardiovascular consequences of emotional states. Psychosomatic Medicine, 64, 714-726.

Goldsmith, H. H., Buss, A. H., Plomin, R., Rothbart, M. K., Thomas, A., Chess, S., Hinde, R.A., \& McCall, R.B. (1987). Roundtable: What is temperament? Four approaches. Child Development, 58, 505-529.

Gorlyn, M., Keilp, J. G., Tryon, W. W., \& Mann, J. J. (2005). Performance test correlates of component factors of impulsivity. Personality and Individual Differences, 38, 15491559. 
Gottfredson, M. R., \& Hirschi, T. (1990). A General Theory of Crime. Stanford, CA: Stanford University Press.

Gough, H.G. (1975). Manual for the California Psychological Inventory. Palo Alto, CA: Consulting Psychologists Press.

Grasmick, H.G., Tittle, T.R., Bursik, R.J. Jr., \& Arneklev, B.J. (1993). Testing the core empirical implications of Gottfredson and Hirschi's General Theory of Crime, Journal of Research in Crime and Delinquency, 30(1), 5-29.

Gray, J. A. (1987). The neuropsychology of emotion and personality. In S. M. Stahl, S. D. Iverson, \& E. C. Goodman (Eds.), Cognitive Neurochemistry. Oxford: Oxford University Press.

Greenberg, L. M. (1998). Test of variables of attention. Journal of Clinical Psychology, 54, 461-476.

Greenberg, L. M. (2002). Test of variables of attention. Journal of Learning Disabilities, 35, 114-120.

Greenberg, L. M., \& Dupuy, T. R. (2000). Test of variables of attention. Journal of Paediatrics and Child Health, 36, 134-138.

Greenberg, L. M., \& Dupuy, T. R. (2001). Test of variables of attention. Neuropsychology, $15,136-144$.

Gross, J.J. (1998). The emerging field of emotion regulation: An integrative review. Review of General Psychology, 2, 271-299.

Gross, J.J., \& Munoz, R.F. (1995). Emotion regulation and mental health. Clinical Psychology: Science and Practice, 2, 151-164. 
Gross, J.J. (2002). Emotion regulation: Affective, cognitive and social consequences. Psychophysiology, 39, 281-291.

Gross, J.J. (2013). Emotion regulation: Taking stock and moving forward. Emotion. Advance online publication. doi: 10.1037/a0032135.

Gross, J.J., Sheepes, G., \& Urry, H.L. (2010). Emotion generation and emotion regulation: A distinction we should make (carefully). Cognition \& Emotion, 25, 765-781.

Gross, J.J., \& Thompson, R.A. (2007). Emotion regulation: Conceptual foundations. In J.J. Gross (Ed.), Handbook of Emotion Regulation (pp. 3-26). New York, NY: Guilford Press.

Guilford, J.S., Zimmerman, W.S., \& Guilford, J.P. (1976). The Guilford-Zimmerman Temperament Survey handbook. San Diego, CA: Edits.

Haden, S., \& Shiva, A. (2008). Trait impulsivity in a forensic inpatient sample: An evaluation of the Barratt Impulsiveness Scale. Behavioral Sciences and the Law, 26, 675-690.

Haden, S. C., \& Shiva, A. (2009). A comparison of factor structures of the Barratt Impulsiveness Scale in a mentally ill forensic inpatient sample. International Journal of Forensic Mental Health, 8, 198-207.

Halperin, J.M., Wolf. L.E., Greenblatt, E., \& Young, J.G. (1991). Subtype analysis of commission errors on the continuous performance test in children. Developmental Neuropsychology, 7, 207-217.

Hancock, M., Tapscott, J.L., \& Hoaken, P.N.S. (2010). Role of executive dysfunction in predicting frequency and severity of violence. Aggressive Behavior, 36, 338-349.

Hanson, R.K., \& Harris, A.J. (2001). A structured approach to evaluating change in sexual offenders. Sexual Abuse, 13(2), 105-122. 
Hanson, R. K., Harris, A. J. R, Scott, T. L., \& Helmus, L. (2007). Assessing the risk of sexual offenders on community supervision: The Dynamic Supervision Project (Corrections research user report 2007-05). Ottawa, Ontario: Public Safety Canada. Retrieved from http://www.publicsafety.gc.ca/res/cor/rep/ fl/crp2007-05-en.pdf

Hanson, R. K., Letourneau, E. J., Olver, M. E., Wilson, R. J., \& Miner, M. H. (2012). Incentives for offender research participation are both ethical and practical. Criminal Justice and Behavior, 39, 1391-1404.

Hare, R.D. (1991). The Hare Psychopathy Checklist-Revised. Toronto, Ontario, Canada: Multi-Health Systems.

Hare, R. D. (2003). The Hare Psychopathy Checklist-Revised (2nd ed.). Toronto, Ontario, Canada: Multi-Health Systems.

Hart, S. D., Forth, A. E., \& Hare, R. D. (1990). Performance of criminal psychopaths on selected neuropsychological tests. Journal of Abnormal Psychology, 99(4), 374-379.

Hay, C., \& Forrest, W. (2006). The development of self-control: examining self-control theory's stability hypothesis. Criminology, 44, 739-774.

Heaton, R. K. (1981). The Wisconsin Card Sorting Test Manual. Odessa: Psychological Assessment Resources Inc.

Heaton, R. K., Chelune, G. J., Talley, J. L., Kay, G. G., \& Curtis, G. (1993). Wisconsin Card Sorting Test (WCST) Manual Revised and Expanded. Odessa: Psychological Assessment Resources Inc.

Henry, B., Caspi, A., Moffitt, T.E., \& Silva, P.A. (1996). Temperamental and familial predictors of violent and non-violent convictions: Age 3 to age 18. Developmental Psychology, 32, 614-623. 
Herrero, O., Escorial, S. \& Colom, R. (2010). Basic executive processes in incarcerated offenders. Personality and Individual Differences, 48, 133-137.

Hertel, P. T., \& Mathews, A. (2011). Cognitive bias modification: Past perspectives, current findings, and future applications. Perspectives on Psychological Science, 6, 521-536.

Higgins, G.E., Jennings, W. G., Tewksbury, R., \& Gibson, C. L. (2009). Exploring the link between low self-control and violent victimization trajectories in adolescents. Criminal Justice and Behavior, 36, 1070-1084.

Hinson, J. M., Jameson, T. L., \& Whitney, P. (2002). Somatic markers, working memory, and decision-making. Cognitive, Affective, \& Behavioral Neuroscience, 2, 341-353.

Hinson, J. M., Jameson, T. L., \& Whitney, P. (2003). Impulsive decision-making and working memory. Journal of Experimental Psychology: Learning, Memory, \& Cognition, 29, 298306.

Holt, D.D., Green, L., \& Myerson, J. (2003). Is discounting impulsive? Evidence from temporal and probability discounting in gambling and non-gambling college students. Behavioural Processes, 64(3), 355- 367.

Hölzel, B.K., Carmody, J., Vangel, M., Congleton, C., Yerramsetti, S.M., Gard, T., \& Lazar, S.W. (2011). Mindfulness practice leads to increases in regional brain gray matter density. Psychiatry Research: Neuroimaging, 191, 36-42.

Homack, S., Lee.D, \& Riccio, C, A. (2005). Test review: Delis-Kaplan executive functions system. Journal of Clinical and Experimental Neuropsychology, 27(5), 599-609.

Hooker, C.I. \& Knight, R.T. (2006). Role of the orbitofrontal cortex in the inhibition of emotion. In D. H. Zald \& S. L. Rauch (Eds.), The Orbitofrontal Cortex, New York: Oxford University Press. 
Horn, N.R., Dolan, M., Elliott, R., Deakin, J.F., \& Woodruff, P.W. (2003). Response inhibition and impulsivity: An fMRI study. Neuropsychologia, 41(14), 1959-1966.

Hortsmann, A., Villringer, A., \& Neumann, J. (2012). Iowa Gambling Task: There is more to consider than long-term outcome. Using a linear equation model to disentangle the impact of outcome and frequency of gains and losses. Frontiers in Neuroscience, 6, 61.

Houben, K. Wiers, R. W., \& Jansen, A. (2011). Getting a grip on drinking behavior: Training working memory to reduce alcohol abuse. Psychological Science, 22, 968-975.

Ireland, J. L., \& Archer, J. (2008). Impulsivity among adult prisoners: A confirmatory factor analysis study of the Barratt Impulsivity Scale. Personality and Individual Differences, 45, 286-292.

Jackson, D.N. (1989). Basic Personality Inventory Manual. Port Huron, MI: Research Psychologists Press.

Jameson, T,L., Hinson, J.M., \& Whitney, P. (2004). Components of working memory and somatic markers in decision making. Psychonomic Bulletin \& Review, 11(3), 515-520.

Jha, A.P., Krompinger, J., \& Baime, M.J. (2007). Mindfulness training modifies subsystems of attention. Cognitive, Affective, \& Behavioral Neuroscience, 7, 109-119.

Jolles, D.D., \& Crone, E.A. (2012). Training the developing brain: a neurocognitive perspective. Frontiers in Human Neuroscience, 6, 76.

Jolles, D.D., van Buchem, M.A., Rombouts, S.A.R.B., \& Crone, E.A. (2012). Practice effects in the developing brain: A pilot study. Developmental Cognitive Neuroscience, 2, S180-S191. 
Kane, M.J., \& Engle, R.W. (2002). The role of the prefrontal cortex in working memory capacity, executive attention, and general fluid intelligence: An individual differences perspective. Psychonomic Bulletin and Review, 9(4), 637-671.

Karbach, J., \& Kray, J. (2009). How useful is executive control training? Age differences in near and far transfer of task-switching training. Developmental Science, 12(6), 978990.

Karbach, J., \& Schubert, T. (2013). Training-induced cognitive and neural plasticity. Frontiers in Human Neuroscience, 7, 48.

Karpinski, A., \& Hilton, J.L. (2001). Attitudes and the implicit association test. Journal of Personality and Social Psychology, 81(5), 774-788.

Kaufman, J., Ross, T.J., Stein, E.A., \& Garavan, H. (2003). Cingulate hypoactivity in cocaine users during a GO-NOGO task as revealed by event-related functional magnetic resonance imaging. The Journal of Neuroscience, 23(21), 7839-7843.

Kenny, D.A. (1975). Cross-lagged panel correlation: A test for spuriousness. Psychological Bulletin, 82(6), 887-903.

Kenny, D. A., \& McCoach, D.B. (2003). Effect of the number of variables on measures of fit in structural equation modeling. Structural Equation Modeling, 10, 333-3511.

Kidd, C., Palmeri, H., \& Aslin, R.N. (2013). Rational snacking: Young children’s decisionmaking on the marshmallow task is moderated by beliefs about environmental reliability. Cognition, 126, 109-114.

Kirby, K.N., \& Finch, J.C. (2010). The hierarchical structure of self-reported impulsivity. Personality and Individual Differences, 48(6), 704-713. 
Knapp, A. \& Clark, M.S. (1991). Some detrimental effects of negative mood on individuals' ability to solve resource dilemmas. Personality and Social Psychology Bulletin, 17, 678-688.

Krain, A.L., Wilson, A.M., Arbuckle, R., Castellanos, X., \& Milhama, M.P. (2006). Distinct neural mechanisms of risk and ambiguity: A meta-analysis of decision-making. Neuroimage, 32, 477-484.

Kratzer, L., \& Hodgins, S. (1999). A typology of offenders: a test of Moffitt's theory among males and females from childhood to age 30. Criminal Behaviour and Mental Health, $9(1), 57-73$.

Kray, J., Karbach, J., Haenig, S., \& Freitag, C. (2012). Can task-switching training enhance executive control functioning in children with attention deficit/-hyperactivity disorder? Frontiers in Human Neuroscience, 5, 180.

Lane, S.D., \& Cherek, D.R. (2000). Analysis of risk taking in adults with a history of highrisk behavior. Drug and Alcohol Dependence, 60, 179-187.

Lapierre, D., Braun, C. M., \& Hodgins, S. (1995). Ventral frontal deficits in psychopathy: Neuropsychological test findings. Neuropsychologia, 33(2), 139-151.

Lecrubier, Y., Braconnier, A., Said, S., \& Payan, C. (1995). The impulsivity rating scale (IRS): Preliminary results. European Psychiatry, 10(7), 331-338.

Lejuez C.W., Aklin, W.M., Jones, H.A., Richards, J.B., Strong, D.R., Kahler, C.W., \& Read, J.P. (2003). The Balloon Analogue Risk Task (BART) differentiates smokers and nonsmokers. Experimental and Clinical Psychopharmacology, 11, 26-33. 
Leotti, L.A. \& Wager, T.D. (2009). Motivational influences on response inhibition measures. Journal of Experimental Psychology: Human Perception and Performance, 36(2), 430-447.

Leth-Steensen, C., Elbaz, Z.K., \& Douglas, V. I. (2000). Mean response times, variability, and skew in the responding of ADHD children: a response time distributional approach. Acta Psychologia, 104, 167-190.

Levi, M.D., Nussbaum, D.A., \& Rich, J.B. (2010). Neuropsychological and personality characteristics of predatory, irritable, and nonviolent offenders: Support for a typology of human aggression. Criminal Justice and Behavior, 37(6), 633-655.

Lezak, M. (1995). Neuropsychological Assessment (3rd ed.). Oxford, England: Oxford University Press.

Lezak, .D., Howieson, D.B., Bigler, E.D., \& Tranel, D. (2012). The neuropsychological examination: Procedures. In Neuropsychological Assessment ( $5^{\text {th }}$ ed.). Madison Avenue, New York: Oxford University Press.

Li, C-SR., Huang, C., Constable, T., \& Sinha, R. (2006). Imaging response inhibition in a stop signal task: Neural correlates independent of signal monitoring and post-response processing. Journal of Neuroscience, 26, 186-192.

Li, C-SR., Milivojevic, V., Kemp, K., Hong, K., \& Sinha, R. (2006). Performance monitoring and stop signal inhibition in abstinent patients with cocaine dependence. Drug and Alcohol Dependence, 85, 205-212.

Lijffijt, M., Bekker, E. M., Quik, E. H., Bakker, J., Kenemans, J. L., \& Verbaten, M. N. (2004). Differences between low and high trait impulsivity are not associated with differences in inhibitory motor control. Journal of Attention Disorders, 8, 25-32. 
Lijffijt, M., Kenemans, J.L., Verbaten, M.N., \& van Engeland, H. (2005). A meta-analytic review of stopping performance in Attention-deficit/hyperactivity disorder: Deficient inhibitory motor control? Journal of Abnormal Psychology, 114(2), 216-222.

Lin, C-H., Chiu, Y.-C., Lee, P.-L., \& Hsieh, J.-C. (2007). Is deck B a disadvantageous deck in the Iowa gambling task? Behavioral and Brain Functions, 3, 16.

Lin, C-H., Song, T-J., Chen, Y-Y., Lee, W-K., \& Chiu, Y-C. (2013). Reexamining the validity and reliability of the clinical version of the Iowa gambling task: evidence from a normal subject group. Frontiers in Psychology, 4, 220.

Linehan, M.M. (1993). Cognitive-behavioural Treatment of Borderline Personality Disorder. New York: Guilford Press.

Loeber, R., Menting, B., Lynam, D.R., Moffitt, T.E., Stouthamer-Loeber, M., Stallings, R., Farrington, D.P., \& Pardini, D. (2012). Findings from the Pittsburgh Youth Study: Cognitive impulsivity and intelligence as predictors of the age-crime curve. Journal of the American Academy of Child and Adolescent Psychiatry, 51(11), 1136-1149.

Logan, G. D. (1994). On the ability to inhibit thought and action: A user's guide to the stop signal paradigm. In D. Dagenbach \& T. H. Carr (Eds.), Inhibitory Processes in Attention, Memory, and Language (pp. 189-239). San Diego: Academic Press.

Logan, G.D., \& Cowan, W.B. (1984). On the ability to inhibit thought and action: A theory of an act of control. Psychological Review, 91(3), 295-327.

Logan, G.D., Schachar, R.J., \& Tannock, R. (1997). Impulsivity and inhibitory control. Psychological Science, 8(1), 60-64.

Logie, R.H. (2012). Cognitive training: Strategies and the multicomponent cognitive system. Journal of Applied Research in Memory and Cognition, 1, 206-207. 
Longshore, D., Turner, S., \& Stein, J.A. (1996). Self-control in a criminal sample: An examination of construct validity. Criminology, 34, 209-228.

Losel, F. (2001). Evaluating the effectiveness of correctional programs: Bridging the gap between research and practice. In G.A. Bernfeld, D.P. Farrington, \& A.W. Leschied (Eds.), Offender Rehabilitation in Practice (pp. 67-92). Chichester: Wiley.

Losel , F., \& Shmucker, M.(2004). Psychopathy, risk taking and attention: A differentiated test of the somatic marker hypothesis. Journal of Abnormal Psychology, 113(4), 522529.

Lowenstein, G., \& Lerner, J. (2003) The role of affect in decision-making. In R. Davidson, K. Scherer \& H. Goldsmith (Eds.), Handbook of Affective Science (pp. 619-642). New York: Oxford University Press.

Luengo, M. A., Carrillo-de-la-Peña, M. T., Otero, J. M., \& Romero, E. (1994). A short-term longitudinal study of impulsivity and antisocial behavior. Journal of Personality and Social Psychology, 66(3), 542-548.

Lykken, D.T. (1995). The Antisocial Personalities. Hillsdale, New Jersey: Lawrence Erlbaum Associates, Inc.

MacLeod, C., \& Bucks, R.S. (2011). Emotion regulation and the cognitive-experimental approach to emotional dysfunction. Emotion Review, 3(1), 62-73.

Magid, V., MacLean, M.G., \& Colder, C.R. (2007). Differentiating between sensation seeking and impulsivity through their mediated relations with alcohol use and problems. Addictive Behaviors, 32, 2046-2061. 
Maia, T.V., \& McClelland, J.L. (2004). Examination of the evidence for the somatic markers hypothesis: What participants really know in the Iowa Gambling Task. Proceedings of the National Academy of Sciences, 101(45), 16075-16080.

Mann, R. E., Webster, S. D., Schofield, C., \& Marshall, W. L. (2004). Approach versus avoidance goals in relapse prevention with sexual offenders. Sexual Abuse: A Journal of Research and Treatment, 16, 65-75.

Marshall, W. L. (1997). The relationship between self-esteem and deviant sexual arousal in non-familial child molesters. Behavior Modification, 21, 86-96.

Mathews, A., \& MacCleod, C. (2005). Cognitive vulnerability to emotional disorders. Annual Review of Clinical Psychology, 1, 167-195.

Mathias, C.W., Dougherty, D.M., Marsh, D.M., Moeller, F.G., Hicks, L.R., Dasher, K., \& BarEli, L. (2002). Laboratory measures of impulsivity: A comparison of women with or without childhood aggression. Psychological Record, 52, 289-303.

Mathias, C.W., Marsh-Richard, D.M., \& Dougherty, D.M. (2008). Behavioral measures of impulsivity and the law. Behavioral Sciences and the Law, 26, 691-707.

Matthies, S., Philipsen, A., \& Svaldi, J. (2012). Risky decision making in adults with ADHD. Journal of Behavior Therapy and Experimental Psychiatry, 43(3), 938-946.

May, A., Hajak, G., Gaenssbauer, S., Steffens, T., Langguth, B., Kleinjung, T., \& Eichhammer, P. (2007). Structural brain alterations following 5 days of intervention: dynamic aspects of neuroplasticity. Cerebral Cortex 17, 205-210.

McCaffrey, R. J., Duff, K., \& Westervelt, H. J. (2000). Practitioner's Guide to Evaluating Change with Intellectual Assessment Instruments. New York: Plenum Press. 
McCaffrey, R.J., Ortega, A., \& Haase, R.F. (1993). Effects of repeated neuropsychological assessments. Archives of Clinical Neuropsychology, 8(6), 519-524.

McCloskey, M. S., \& Coccaro, E. F. (2003). Questionnaire and interview measures of aggression in adults. In E. F. Coccaro (Ed.), Aggression: Psychiatric Assessment and Treatment (pp. 167-193). New York: Marcel Dekker.

McDonald, J., Schleifer, L., Richards, J.B., \& de Wit, H. (2003). Effects of THC on behavioral measures of impulsivity in humans. Neuropsychopharmacology, 28(7), $1356-1365$.

McHale, T.J. (2010). Delayed Maturation of Neuropsychological Abilities in ADHD: A Developmental Comparison Between Children, Adolescents, and Adults. (Unpublished doctoral dissertation). Fielding Graduate University, Santa Barbara, California.

McMurran, M., Blair, M., \& Egan, V. (2002), An investigation of the correlations between aggression, impulsiveness, social problem-solving, and alcohol use. Aggressive Behavior, 28, 439-445.

Meier, N.M., Perrig, W., \& Koenig, T. (2012). Neurophysiological correlates of delinquent behaviour in adult subjects with ADHD. International Journal of Psychophysiology, Advance online publication. doi: 10.1016/j.ijpsycho.2011.12.011.

Miller L. (1992). Impulsivity, risk-taking, and the ability to synthesize fragmented information after frontal lobectomy. Neuropsychologia, 30, 69-79.

Mitchell, D.G.V., Colledge, E., Leonard, A. \& Blair, R.J.R. (2002). Risky decisions and response reversal: Is there evidence of orbitofrontal cortex dysfunction in psychopathic individuals? Neuropsychologia, 40, 2013-2022. 
Miyake, A., \& Friedman, N.P. (2012). The nature and organization of individual differences in executive functions: Four general conclusions. Current Directions in Psychological Science, 21(1), 8-14.

Mobini, S., Grant, A., Kass, A.E., \& Yeomans, M.R. (2007). Relationships between functional and dysfunctional impulsivity, delay discounting and cognitive distortions. Personality and Individual Differences, 43, 1517-1528.

Moeller, G., Barratt, E., Dougherty, D., Schmitz, J., \& Swann, A. (2001). Psychiatric aspects of impulsivity. American Journal of Psychiatry, 158, 1783-1793.

Moeller, F. G., Dougherty, D, M., Steinberg, J.L., Swann, A, C., Silverman, P.B., Ruiz, P., \& Barratt, E. S. (2002). Heavy "Ecstasy" use is associated with increased impulsivity. Addictive Disorders and Their Treatment, 1(2), 47-52.

Moffitt, T. E. (1993). Adolescence-Limited and Life-Course-Persistent antisocial behavior: A developmental taxonomy. Psychological Review, 100(4), 674-701.

Moffitt, T.E., Caspi, A., Dickson, N., Silva, P., \& Stanton, W. (1996). Childhood-onset versus adolescent-onset antisocial conduct problems in males: Natural history from ages 3 to 18 years. Development and Psychopathology, 8, 399-424.

Moffitt, T.E., Caspi, A., Harrington, H., \& Milne, B.J. (2002). Males on the life-coursepersistent and adolescence-limited antisocial pathways: Follow-up at age 26 years. Development and Psychopathology, 14, 179-207.

Moffitt, T.E., Lynam, D., \& Silva, P.A. (1994). Neuropsychological tests predicting persistent male delinquency. Criminology, 32(2), 277-288.

Mogg, K., \& Bradley, B. P. (2005). Attentional bias in generalized anxiety disorder versus depressive disorder. Cognitive Therapy and Research, 29, 2945. 
Monterosso, J., \& Ainslie, G. (1999). Beyond discounting: Possible experimental models of impulse control. Psychopharmacology, 146, 339-347.

Morgan, A.B., \& Lilienfeld, S.O. (2000). A meta-analytic review of the relation between antisocial behavior and neuropsychological measures of executive function. Clinical Psychology Review, 20(1), 113-136.

Morris, S. B., \& DeShon, R. P. (2002). Combining effect size estimates in meta-analysis with repeated measures and independent-groups designs. Psychological Methods, 7, 105125.

Muraven, M., \& Baumeister, R.F. (2000). Self-regulation and depletion of limited resources: Does self-control resemble a muscle? Psychological Bulletin, 126(2), 247-259.

Muraven, M., Baumeister, R.F., \& Tice, D.M. (1999). Longitudinal improvement of selfregulation through practice: Building self-control strength through repeated exercise. Journal of Social Psychology, 139, 446-457.

Newman, J.P., Patterson, C.M., \& Kosson, D.S. (1987). Response perseveration in psychopaths. Journal of Abnormal Psychology, 96, 145-8.

Newman, J. P., Widom, C. S., \& Nathan, S. (1985). Passive avoidance in syndromes of disinhibition: Psychopathy and extraversion. Journal of Personality and Social Psychology, 48, 1316-1327.

Nigg, J.T. (2000). On inhibition/disinhibition in developmental psychopathology: views from cognitive and personality psychology and a working inhibition taxonomy. Psychological Bulletin, 126, 220-246.

Noel, X., Van Der Linden, M., \& Bechara, A. (2006). The neurocognitive mechanisms of decision-making, impulse control and loss of willpower. Psychiatry, 3(5), 30-41. 
Norman, W., \& Shallice, T. (1986). Attention to action. In R.J. Davidson, G.E. Schwartz \& D. Shapiro (Eds.), Consciousness and Self-regulation: Advances in Research and Theory (pp.1-18). New York: Plenum Press.

Norris, G. \& Tate, R. L. (2000). The behavioural assessment of the dysexecutive syndrome (BADS): Ecological, concurrent and construct validity. Neuropsychological Rehabilitation, 10(1), 33-45.

Nunes, K., \& Cortoni, F. (2006). Estimating Risk of Dropout and Expulsion from Correctional Programmes (No. R-177). Ottawa, Ontario: Correctional Service of Canada.

O’Brien, B.S., \& Frick, P.J. (1996), Reward dominance: associations with anxiety conduct problems, and psychopathy in children. Journal of Abnormal Child Psychology, 24, $223-240$.

Ochsner, K. N., \& Gross, J. J. (2004). Thinking makes it so: A social cognitive neuroscience approach to emotion regulation (pp. 221-255). In R. Baumeister \& K. Vohs (Eds.), The Handbook of Self-regulation. New York: Guilford Press.

Ochsner, K. N., Ray, R. D., Robertson, E. R., Cooper, J. C., Chopra, S., Gabrieli, J. D. E., \& Gross, J. J. (2004). For better or for worse: Neural systems supporting the cognitive down- and up-regulation of negative emotion. Neuroimage, 23(2), 483-499.

Ogilvie, J.M., Stewart, A.L., Chan, R.C.K., \& Shum, D.H.K. (2011). Neuropsychological measures of executive and antisocial behaviour: A meta-analysis. Criminology, 49(4), $1063-1107$. 
Ogloff, J. R. P. (1997). Legal perspectives on the concept of "impulsivity.” In C. D. Webster \& M. A. Jackson (Eds.), Impulsivity: Theory, Assessment, and Treatment (pp. 63-81). New York: Guilford.

Olver, M.E., Wong, S.C.P., Nicholaichuk, T., \& Gordon, A. (2007). The validity and reliability of the Violence Risk Scale-Sexual Offender version: Assessing sex offender risk and evaluating therapeutic change. Psychological Assessment, 19(3), 318-329.

Ouellette, J. A., \& Wood, W. (1998). Habit and intention in everyday life: The multiple processes by which past behavior predicts future behavior. Psychological Bulletin, 124, 54-74.

Overtoom C.C.E., Kenemans J.L., Verbaten M.N., Kemmer C., van der Molen M.W., \& van Engeland, H. (2002). Inhibition in children with attention-deficit hyperactivity disorder: A psychophysiological study of the stop task. Biological Psychiatry, 51(8), 668-676.

Ownsworth, T.L., McFarland, K., \& Mc Young, R.D. (2000). Self-awareness and psychosocial functioning following acquired brain injury: An evaluation of a group support programme. Neuropsychological Rehabilitation: An International Journal, $10(5), 465-484$

Parker, J.D.A., \& Bagby, R.M. (1997). Impulsivity in adults: A critical review of measurement approaches. In C.D. Webster, CD \& M.A. Jackson (Eds.), Impulsivity: Theory, Assessment, and Treatment (pp.142-157). New York: Guilford Press.

Parry, C.J., \& Lindsay, W.R. (2003). Impulsiveness as a factor in sexual offending by people with mild intellectual disability. Journal of Intellectual Disability Research, 47(6), $483-487$. 
Patrick, C.J., Fowles, D.C., \& Krueger, R.F. (2009). Triarchic conceptualization of psychopathy: Developmental origins of disinhibition, boldness and meanness. Development and Psychopathology, 21, 913-938.

Patton, J. H., Stanford, M. S., \& Barratt, E. S. (1995). Factor structure of the Barratt Impulsiveness Scale. Journal of Clinical Psychology, 51, 768-774.

Pessoa, L. (2005). To what extent are emotional visual stimuli processed without attention and awareness? Current Opinion in Neurobiology, 15, 188-196.

Pessoa, L. (2009). How do emotion and motivation direct executive function? Trends in Cognitive Sciences, 13, 160-166.

Pessoa, L., McKenna, M., Gutierrez, E., \& Ungerleider, L.G. (2002). Neural processing of emotional faces requires attention. Proceedings of the National Academy of Sciences, 99, 11458-11463.

Pham, T.H., Vanderstukken, O., Philippot, P., \& Vanderlinden, M. (2003). Selective attention and executive functions deficits among criminal psychopaths. Aggressive Behavior, 5, $393-405$.

Piquero, A.R. (2001). Testing Moffitt's neuropsychological variation hypothesis for the prediction of life-course persistent offending. Psychology, Crime and Law, 7, 193-216.

Piquero, A.R., \& Brezina, T. (2001). Testing Moffitt's account of adolescence-limited delinquency. Criminology, 39, 353-370.

Piquero, A.R., Diamond, B., Jennings, W.G., \& Reingle, J.M. (2013). Adolescence-limited offending. In C.L. Gibson and M.D. Krohn (Eds.), Handbook of Life-course Criminology: Emerging Trends and Directions for Future Research (pp. 129-142). New York: Springer Science and Business Media. 
Piquero, A.R., \& Moffitt, T.E. (2011). Explaining the facts of crime: How the developmental taxonomy replies to Farrington's Invitation. In D.P Farrington (Ed), Integrated Developmental and Life-course Theories of Offending: Advances in Criminological Theory (pp. 51-72). New Brunswick, New Jersey: Transaction Publishers.

Piquero, A. R., \& Tibbetts, S. G. (1999). The impact of pre/perinatal disturbances and disadvantaged familial environment in predicting criminal offending. Studies on Crime and Crime Prevention, 8,52-70.

Polaschek, D. L. L. (2009). Te Whare Manaakitanga/Rimutaka Violence Prevention Unit Evaluation Report VI. Evaluating the predictive validity of psychometric assessment measures: Treatment completion and recidivism outcomes (prospective evaluation sample). Wellington, NZ: Unpublished report for the New Zealand Department of Corrections.

Polaschek, D.L.L. (2010a). Treatment non-completion in high-risk offenders: looking beyond criminal risk and criminogenic needs. Psychology, Crime \& Law, 16(6), 525-540.

Polaschek, D. L. L. (2010b). Treatment, change, desistance and re-entry: The sequel. Understanding and measuring treatment change. Keynote address at Department of Corrections Psychological Service conference Sept 2010.

Polaschek, D. L. L., \& Collie, R. M. (2004). Rehabilitating serious violent adult offenders: An empirical and theoretical stocktake. Psychology, Crime and Law, 19(3), 321-334.

Polaschek, D.L.L., \& Kilgour, T.G. (2013). New Zealand's special treatment units: the development and implementation of intensive treatment for high-risk male prisoners. Psychology, Crime and Law. Advance online publication. doi:

10.1080/1068316X.2013.759004. 
Posner, M. I., \& Rothbart, M. K. (2000). Developing mechanisms of self-regulation. Development and Psychopathology, 12, 427-441.

Prentky, R. A., \& Knight, R. A. (1986). Impulsivity in the lifestyle and criminal behavior of sexual offenders. Criminal Justice and Behavior, 13, 141-164.

Proschaska, L.O., DiClemente, C.C., \& Norcross, J.C. (1992). In search of how people change: Applications to addictive behaviors. The American Psychologist, 47(9), 11021114.

Quigley B.M., \& Tedeschi, J.T. (1996). Mediating effects of blame attributions on feelings of anger. Personality and Social Psychology Bulletin, 22, 1280-1288.

Raaijmakers, M.A.J., Smidts, D.P., Sergeant, J.A., Maassen, G.H., Posthumus, J.A., van Engeland, H., \& Matthys, W. (2008). Executive functions in preschool children with aggressive behaviour: Impairments in inhibitory control. Journal of Abnormal Child Psychology, 36, 1097-1107.

Raine, A., Brennan, P., Mednick, B., \& Mednick, S.A. (1996). High rates of violence, crime, academic problems, and behavioral problems in males with both early neuromotor deficits and unstable family environments. Archives of General Psychiatry, 53, 544549.

Raine, A., Lencz, T., Bihrle, S., LaCasse, L., \& Colletti, P. (2000). Reduced prefrontal gray matter volume and reduced autonomic activity in antisocial personality disorder. Archives of General Psychiatry, 57, 119-127.

Raine, A., Moffitt, T.E., Caspi, A., Loeber, R., Stouthamer-Loeber, M., \& Lynam, D. (2005). Neurocognitive and psychosocial deficits in life-course persistent offenders. Journal of Abnormal Psychology, 114, 38-49. 
Rath, J.F., Simon, D., Langenbahn, D.M., Sherr, R.L., \& Diller, L. (2003). Group treatment of problem solving deficits in patients with traumatic brain injury: A randomized outcome study. Neuropsychological Rehabilitation, 13(4), 461-488.

Ray, J.V., Jones, S., Loughran, T.A., \& Jennings, W.G. (2013). Testing the stability of selfcontrol: Identifying unique developmental patterns and associated risk factors. Criminal Justice and Behavior, 40, 588-607.

Ray, R.D., Wilhelm, F.H., \& Gross, J.J. (2008). All in the mind's eye? Anger rumination and reappraisal. Journal of Personality and Social Psychology, 94(1), 133-145.

Ready, R. E., Stierman, L. \& Paulsen, J.S. (2001). Ecological validity of neuropsychological and personality measures of executive functions. The Clinical Neuropsychologist, $15(3), 314-323$.

Retz, W., Retz-Junginger, P., Supprian, T., Thome, J., \& Rosler, M. (2004). Association of serotonin transporter promoter gene polymorphism with violence: relation with personality disorders, impulsivity, and childhood ADHD psychopathology. Behavioral Sciences and the Law, 22(3), 415-425.

Revelle, W., Anderson, K. J. \& Humphreys, M. S. (1987). Empirical tests and theoretical extensions of arousal based theories of personality. In J. Strelau \& H. J. Eysenck (Eds.), Personality Dimensions and Arousal (pp. 17-36). London: Plenum.

Reynolds, C. R. (2002). Comprehensive Trail Making Test: Professional manual. Austin, TX: Pro Ed.

Reynolds, B. (2006). A review of delay-discounting research with humans: relations to drug use and gambling. Behavioural Pharmacology, 17(8), 651-667. 
Reynolds, C.R., \& Horton, A.M. (2008). Assessing executive functions: A life-span perspective. Psychology in the Schools, 45(9), 875-892.

Reynolds, B., Ortengren, A., Richards, J.B., \& deWit, H. (2006). Dimensions of impulsive behavior. Personality and Individual Differences, 40, 305-315.

Reynolds, B., Richards, J.B., Horn, K., \& Karraker, K. (2004). Delay discounting and probability discounting as related to cigarette smoking status in adults. Behavioral Processes, 65, 35-42.

Riccio, C. A., Reynolds, C.R., \& Lowe, P.A. (2001). Clinical Applications of Continuous Performance Tests: Measuring Attention and Impulsive Responding in Children and Adults. New York: John Wiley \& Sons, Inc.

Rieger, M., \& Gauggel, S. (1999). Inhibitory after effects in the stop signal paradigm. British Journal of Psychology, 90(4), 509-518.

Riggs, N.R., Greenberg, M.T., Kusche, C.A., \& Pentz, M.A. (2006). The meditational role of neurocognition in the behavioral outcomes of a socio-emotional prevention program in elementary school students: Effects of the PATHS curriculum. Prevention Science, 7, 91-102.

Robbins, T.W., Gillan, C.M., Smith, D.G., de Wit, S., \& Ersch, K.D. (2012). Neurocognitive endophenotypes of impulsivity and compulsivity: Towards dimensional psychiatry. Trends in Cognitive Sciences, 16(1), 81-91.

Rodriguez-Fornells, A., Lorenzo-Sera, U., \& Andres-Pueyo, A. (2002). Are high-impulsive people more motor disinhibited in the presence of incentive? Personality and Individual Differences, 32, 661-683. 
Romine, C. B., \& Reynolds, C. R. (2005). A model of the development of frontal lobe functioning: Findings from a meta-analysis. Applied Neuropsychology, 12(4), 190201.

Rothbart, M. K., Ahadi, S. A., \& Evans, D. E. (2000). Temperament and personality: Origins and outcomes. Journal of Personality and Social Psychology, 78, 122-135.

Rothwell, N.A., La Vigna, G.W., \& Willis, T.J. (1999). A non-aversive rehabilitation approach for people with severe behavioral problems resulting from brain injury. Brain Injury, 13(7), 521-533.

Rubia K., Russell T., Overmeyer S., Brammer M. J., Bullmore E. T., Sharma T., Simmons, A., Williams, S.C., Giampietro, V, Andrew, C.M., \& Taylor, E. (2001). Mapping motor inhibition: conjunctive brain activations across different versions of go/no-go and stop tasks. Neuroimage, 13, 250-261.

Ruiz , M.A., Skeem, J.L., Poythress, N.G., Douglas, K.S. \& Lilienfeld, S.O. (2010): Structure and correlates of the Barratt Impulsiveness Scale (BIS-11) in offenders: Implications for psychopathy and externalizing pathology. International Journal of Forensic Mental Health, 9(3), 237-244.

Rusting, C.L., \& Nolen-Hoeksema, S. (1998). Regulating responses to anger: Effects of rumination and distraction on angry mood. Journal of Personality and Social Psychology, 74(3), 790-803.

Sacco, K., Cauda, F., D’Agata, F., Duca, S., Zettin, M., Virgilio, R., Nascimbeni, A., Belforte, G., Eula, G., Gastaldi, L., Appendino, S., \& Geminiani, G. (2011). A combined robotic and cognitive training for locomotor rehabilitation: evidences of cerebral 
functional reorganization in two chronic traumatic brain injured patients. Frontiers in Human Neuroscience, 5, 146.

Salminen, T., Strobach, T., \& Schubert, T. (2012). On the impact of working memory training on executive functioning. Frontiers in Human Neuroscience, 6, 166.

Salthouse, T.A., Atkinson, T.M., \& Berish, D.E. (2003). Executive functioning as a potential mediator of age-related cognitive decline in normal adults. Journal of Experimental Psychology: General, 132, 566-594.

Sanchez-Cubillo, I., Perianez, J.A., Adrover-Roig, D., Rodriguez-Sanchez, J.M., Rios-Lago, M., Tirapu, J., \& Barcelo, F. (2009). Construct validity of the Trail Making Test: Role of task-switching, working memory, inhibition/interference control, and visuomotor abilities. Journal of the International Neuropsychological Society, 15, 438-450.

Sandford, J.A., \& Turner, A. (1995). Manual for the Integrated Visual and Auditory Continuous Performance Test. Richmond, VA: Brain Train.

Schachar, R. J., Chen, S., Logan, G. D., Ornstein, T. J., Crosbie, J., Ickowicz, A., \& Pakulak, A. (2004). Evidence for an error monitoring deficit in attention deficit hyperactivity disorder. Journal of Abnormal Child Psychology, 32, 285-293.

Schachar, R.J., Mota, V.L., Logan, G.D., Tannock, R., \& Klim, P. (2000). Confirmation of an inhibitory control deficit in attention-deficit/hyperactivity disorder. Journal of Abnormal Child Psychology, 28(3), 227-235.

Schachar R. J., Tannock R., Logan G. D. (1993). Inhibitory control, impulsiveness, and attention deficit hyperactivity disorder. Clinical Psychology Review, 13, 721-739. 
Schmidt, C.A., Fallon, A.E., \& Coccaro, E.F. (2004). Assessment of behavioural and cognitive impulsivity: Development and validation of the lifetime history of impulsive behaviours interview. Psychiatry Research, 126(2), 107-121.

Schneider, W. \& R. M. Shiffrin. (1977). Controlled and automatic human information processing: Detection, search, and attention. Psychological Review, 84, 1-66.

Schretlen, D.J. Tester, S.M., Winicki, J.M., Pearlson, G.D., \& Gordon, B. (2008). Frequency and bases of abnormal performance by healthy adults on neuropsychological testing. Journal of the International Neuropsychological Society, 14, 436-445.

Schuch, S., \& Koch, I. (2003). The role of response selection for inhibition of task sets in task shifting. Journal of Experimental Psychology, 29(1), 92-105.

Seager, J.A. (2005). Violent men: the importance of impulsivity and cognitive schema. Criminal Justice and Behavior, 32, 26-49.

Shamay-Tsoory, S.G., Harari, H., Szepsenwol, O., \& Levkovitz, Y. (2009). Neuropsychological evidence of impaired cognitive empathy in euthymic bipolar disorder. Journal of Neuropsychiatry and Clinical Neurosciences, 21(1), 59-67.

Shapiro, D. (1981). Autonomy and Rigid Character. New York: Basic Books.

Sharma, D., Albery, I.P., \& Cook, C. (2001). Selective attentional bias to alcohol related stimuli in problem drinkers and non-problem drinkers. Addiction, 96, 285-95.

Shipstead, Z., Hicks, K.L., \& Engle, R.W. (2012). Cogmed working memory training: Does the evidence support the claims? Journal of Applied Research in Memory and Cognition, 1, 185-193.

Shuster, J., \& Toplak, M. E. (2009). Executive and motivational inhibition: Associations with measures of everyday inhibition. Consciousness and Cognition, 18, 471-480. 
Siemer, M., Mauss, I., \& Gross, J.J. (2007). Same situation-different emotions: How appraisals shape our emotions. Emotion, 7, 592-600.

Silva, P.A. (1990). The Dunedin Multidisciplinary Health and Development Study: a 15 year longitudinal study. Paediatric and Perinatal Epidemiology, 4(1), 76-107.

Silverberg, N.D., \& Millis, S.R. (2009). Impairment versus deficiency in neuropsychological assessment: Implications for ecological validity. Journal of the International Neuropsychological Society, 15, 94-102.

Singh, V., \& Khan, A. (2009). Heterogeneity in choices on the Iowa Gambling Task: Preference for infrequent-high magnitude punishment.

Smith, S.R., Servesco, A, M., Edwards, J.W., Rahban, R., Barazani, S., Nowinski, L.A., Little, J.A., Blazer, A.L., \& Green, J.G. (2008). Exploring the validity of the comprehensive trail-making test. The Clinical Neuropsychologist, 22(3), 507-518.

Smith, P., \& Waterman, M. (2004). Processing bias for sexual material: The emotional Stroop and sexual offenders. Sexual Abuse: A Journal of Research and Treatment, 16, 163171.

Solanto, M.V., Abikoff, H., Sonuga-Barke, E., Schachar, R., Logan, G.D., Wigal, T., Hechtman, L., Hinshaw, S., \& Turkel, E. (2001). The ecological validity of delay aversion and response inhibition as measures of impulsivity in AD/HD: A supplement to the NIMH multimodal treatment study of AD/HD. Journal of Abnormal Child Psychology, 29(3), 215-228.

Soper, H. (2001). Subtypes of Attention Deficit Hyperactivity Disorder. Unpublished manuscript. Ventura, CA: Developmental Neuropsychology Laboratory. 
Spielberger, C. D. (1999). State Trait Anger Expression Inventory-2: Professional manual. Odessa, FL: Psychological Assessment Resources.

Spreen, O., \& Strauss, E. (1998). A Compendium of Neuropsychological Tests. New York: Oxford University Press.

Steinberg, L., Albert, D., Cauffman, E., Banich, M., Graham, S., \& Woolard, J. (2008). Age differences in sensation-seeking and impulsivity as indexed by behaviour and selfreport: Evidence for a dual-systems model. Developmental Psychology, 44, 17641778.

Steingroever, H., Wetzels, R., Horstmann, A., Neumann, J., \& Wagenmakers, E-J. (2013). Performance of healthy participants on the Iowa Gambling Task. Psychological Assessment, 25(1), 180-193.

Stocco, A., Fum, D., \& Napoli, A. (2009). Dissociable processes underlying decisions in the Iowa Gambling Task: A new integrative framework. Behavioural and Brain Functions, 5(1), 1-12.

Stormark, K.M., Laberg, J.C., Nordby, H., \& Hugdahl, K. (2000). Alcoholics’ selective attention to alcohol stimuli: Automated processing? Journal of Studies on Alcohol, 61, $18-23$.

Strack, F. \& Deutsch,R. (2004). Reflective and impulsive determinants of social behaviour. Personality and Social Psychology Review, 8(3), 220-247.

Stroop, J. R. (1935). Studies of interference in serial verbal reactions. Journal of Experimental Psychology, 12, 643-662.

Stuss, D.T., \& Knight, R.T. (2002) . Principles of Frontal Lobe Function. New York: Oxford University Press. 
Suhr, J.A., \& Tsanadis, J. (2007). Affect and personality correlates of the Iowa Gambling Task. Personality and Individual Differences, 43, 27-36.

Sung, H-E., \& Richter, L. (2006). Contextual barriers to successful reentry of recovering drug offenders. Journal of Substance Abuse Treatment, 31, 365-374.

Swann, A.C., Anderson, J., Dougherty, D.M. \& Moeller, F.G. (2001). Measurement of interepisode impulsivity in bipolar disorder: A preliminary report. Psychiatry Research, 101, 195-197.

Swann, A.C., Bjork, J.M., Moeller, F.G. \& Dougherty, D.M. (2002). Two models of impulsivity: relationship to personality traits and psychopathology. Biological Psychiatry, 51(12), 988-994.

Swann, A.C., Lijffijt, M., Lane, S.D., Kjome, K.J., Steinberg, J.L., \& Moeller, F.G. (2011). Criminal conviction, impulsivity, and course of illness in bipolar disorder. Bipolar Disorder, 13(2), 173-181.

Tabachnick, B. G., \& Fidell, L. S. (2001). Using Multivariate Statistics (4th ed.). Needham Heights, MA: Allyn \& Bacon.

Tchanturia, K., Anderluh, M.B., Moriss, R.G., Rabe-Hesketh, S., Collier, D.A., Sanchez, P., \& Treasure, J.L. (2004). Cognitive flexibility in anorexia nervosa and bulimia nervosa. Journal of the International Neuropsychological Society, 10, 513-520.

Tellegen, A. (1982). Multidimensional Personality Questionnaire manual. Minneapolis, MN: University of Minnesota Press.

Teuber, H.L. (1972). Unity and diversity of frontal lobe functions. Acta Neurobiologiae Experimentalis, 32, 615-656. 
Tibbetts, S., \& Piquero, A. (1999). The influence of gender, low birth weight and disadvantaged environment on predicting early onset of offending: A test of Moffitt's interactional hypothesis. Criminology, 37, 843-878.

Tice, D., M., Bratslavksy, E., \& Baumeister, R.F. (2001). Emotional distress takes precedence over impulse control: If you feel bad, do it! Journal of Personality and Social Psychology, 80(1), 53-67.

Thornton, D. (1985). Rate of offending, risk-evaluation and risk-preference. Personality and Individual Differences, 6(1), 127-128.

Toplak, M. E., Sorge, G. B., Benoit, A., West, R. F., \& Stanovich, K. E. (2010). Decisionmaking and cognitive abilities: A review of associations between Iowa Gambling Task performance, executive functions, and intelligence. Clinical Psychology Review, 30, $562-581$.

Tranel, D., Bechara, A., \& Denburg, N.L. (2002). Asymmetric functional roles of right and left ventromedial prefrontal cortices in social conduct, decision-making, and emotional processing. Cortex, 38(4), 589-612.

Vaidya, J.G., Latzman, R.D., Markon, K.E., \& Watson, D. (2010). Age differences on measures of disinhibition during young adulthood. Personality and Individual Differences, 48, 815-820.

Van de Laar, M.C., van den Wildenberg, W.P., van Boxtel, G.J., \& van der Molen. M,W. (2011). Lifespan changes in global and selective stopping and performance adjustments. Frontiers in Psychology, 2, 1-12. 
Van Rensburg, J., Berry, S., Rutherford B., Williams, K., Whitehead, P., \& van Rensburg, P. (2009). Special Treatment Unit Operations Manual. Unpublished report, Department of Corrections, Wellington, New Zealand.

Verbruggen, F., \& Logan, G.D. (2009). Proactive adjustments of response strategies in the stop signal paradigm. Journal of Experimental Psychology: Human Perception and Performance, 35(3), 835-854.

Verbruggen, F., Logan, G.D., Liefooghe, B., \& Vandierendonck, A. (2008). Short-term aftereffects of response inhibition: Repetition priming or between-trial control adjustments? Journal of Experimental Psychology: Human Perception and Performance, 34, 413-426.

Verbruggen, F., Logan, G. D., \& Stevens, M. A. (2008). STOP-IT: Windows executable software for the stop-signal paradigm. Behavior Research Methods, 40(2), 479-483.

Verdejo-Garcia, A., Bechara, A., Recknor. E., \& Perez-Garcia, M. (2006). Executive dysfunction in substance dependent individuals during drug use and abstinence: An examination of the behavioural, cognitive and emotional correlates of addiction. Journal of the International Neuropsychological Society, 12, 405-415.

Verdejo-Garcia, A., \& Pérez-Garcia, M. (2007). Ecological assessment of executive functions in substance dependent individuals. Drug and Alcohol Dependence, 90, 48-55.

von Cramon, D. Y., Matthes-von Cramon, G., \& Mai, N. (1991). Problem solving deficits in brain injured patients. A therapeutic approach. Neuropsychological Rehabilitation, 1, 45-64.

Wagar, B.M., \& Dixon, M. (2006). Affective guidance in the Iowa Gambling Task. Cognitive, Affective and Behavioral Neuroscience, 6(4), 277-290. 
Walters, G.D. (2000). Outcome expectancies for crime: Their relationship to fear and the negative consequences of criminal involvement. Legal and Criminological Psychology, 5, 261-272.

Walters, G.D. (2003). Changes in outcome expectancies and criminal thinking following a brief course of psychoeducation. Personality and Individual Differences, 35, 691-701.

Ward, T., \& Beech, A. (2006). An integrated theory of sexual offending. Aggression and Violent Behavior, 11, 44-63.

Ward, T., Day, A., Howells, K., \& Birgden, A. (2004). The multifactor offender readiness model. Aggression and Violent Behavior, 9, 645-673.

Ward, T., \& Beech, A. (2006). An integrated theory of sexual offending. Aggression and Violent Behavior, 11, 44-63.

Ward, T., \& Gannon, T. A. (2006). Rehabilitation, etiology, and self-regulation: The comprehensive good lives model of treatment for sexual offenders. Aggression and Violent Behavior, 11, 77-94.

Ward, T., \& Hudson, S. M. (2000). A self-regulation model of relapse prevention. In D. R. Laws, S. M. Hudson \& T. Ward (Eds.), Remaking Relapse Prevention with Sex Offenders: A Sourcebook (pp. 79-101). Newbury Park: CA: Sage.

Ward, T., Polaschek, D., \& Beech, A. R. (2005). Theories of Sexual Offending. Chichester: John Wiley \& Sons Ltd.

Watt, J.D., \& Vodanovich, S.J. (1992). Relationship between boredom proneness and impulsivity. Psychological Reports, 70, 688-690. 
Webster, C., Douglas, K., Eaves, D., \& Hart, S. (1997). HCR-20: Assessing Risk for Violence (Version 2). Burnaby, British Columbia, Canada: Mental Health, Law and Policy Institute, Simon Fraser University.

Wechsler, D. (1999). Wechsler Abbreviated Scale of Intelligence. The Psychological Corporation: Harcourt Brace \& Company, New York.

White, J., Moffitt, T.E., Caspi, A., Jeglum-Bartusch, D., Needles, D., \& Stouthamer-Loeber, M. (1994). Measuring impulsivity and examining its relation to delinquency. Journal of Abnormal Psychology, 103, 192-205.

Whiteside, S.P., \& Lynam, D.R. (2001). The Five Factor Model and impulsivity: using a structural model of personality to understand impulsivity. Personality and Individual Differences, 30, 669-689.

Whitmer, A.J., \& Banich, M.T. (2010). Trait rumination and inhibitory deficits in long-term memory. Cognition \& Emotion, 24, 168-179.

Wiers, R.W., Gladwin, T.E., Hofmann, W., Salemink , E., \& Ridderinkhof, K.R. (2013). Cognitive bias modification and cognitive control training in addiction and related psychopathology: Mechanisms, clinical perspectives, and ways forward. Clinical Psychological Science, 1(2), 192-212.

Wilkowski, B.M. (2012). Responding to social signals for response inhibition: A psychological process underlying trait anger. Social Psychological and Personality Science, 3(1), 72-79.

Wilkowski, B.M., \& Robinson, M.D. (2007). Keeping one's cool: Trait anger, hostile thoughts and the recruitment of limited capacity control. Personality and Social Psychology Bulletin, 33, 1201-1213. 
Wilkowski, B. M., \& Robinson, M. D. (2008). The cognitive basis of trait anger and reactive aggression: An integrative analysis. Personality and Social Psychology Review, 12, 321.

Wilkowski, B. M., \& Robinson, M. D. (2010). The anatomy of anger: An integrative cognitive model of trait anger and reactive aggression. Journal of Personality, 78, 938.

Williams, B. R., Ponesse, J. S., Schachar, R. J., Logan, G. D., \& Tannock, R. (1999). Development of inhibitory control across the life span. Developmental Psychology, 35, 205-213.

Williams, J.M.G., Watts, F.N, MacLeod, C., \& Mathews, A. (1997). Cognitive Psychology and Emotional Disorders (2 ${ }^{\text {nd }}$ ed.). Chichester, UK: Wiley.

Wilson, B. A. (1993). Ecological validity of neuropsychological assessment: Do neuropsychological indexes predict performance in everyday activities? Applied and Preventive Psychology, 2, 209-215.

Wilson, N. J. (2004, July). New Zealand high-risk offenders: Who are they and what are the issues in their management and treatment? Retrieved from New Zealand Department of Corrections website: http://www.corrections.govt.nz/research/high-riskoffenders.html.

Winchel, R.M. \& Stanley, M. (1991). Self-injurious behavior: A review of the behavior and biology of self-mutilation. American Journal of Psychiatry, 148(3), 306-317.

Winstanley, C.A., Eagle, D.M., \& Robbins, T.W. (2006). Behavioral models of impulsivity in relation to ADHD: Translation between clinical and preclinical studies. Clinical Psychology Review, 26(4), 379-395. 
Wong, S.C.P. (2000). Psychopathic offenders. In S. Hodgins and R. Muller-Isberner (Eds.), Violence, Crime and Mentally Disordered Offenders: Concepts and Methods for Effective Treatment and Prevention (pp. 87-112). Chichester: Wiley.

Wong, S.C.P., \& Gordon, A. (2000). The Violence Risk Scale. University of Saskatchewan, Canada.

Wong, S.C.P., \& Gordon, A. (2006). The validity and reliability of the Violence Risk Scale: a treatment-friendly violence risk assessment tool. Psychology, Public Policy and Law, 12(3), 279-309.

Wong, S.C.P., \& Olver, M, E. (2010). Two treatment and change-oriented risk assessment tools: The violence risk scale and violence risk scale-sexual offender version. In R.K. Otto \& K.S. Douglas (Eds.), Handbook of Violence Risk Assessment (pp. 121-146). Madison Avenue, New York: Taylor \& Francis Group.

Wyder, M., \& De Leo, D. (2007). Behind impulsive suicide attempts: indications from a community study. Journal of Affective Disorders, 104 (1-3), 167-173.

Yechiam, E., Kanz, J.E., Bechara, A., Stout, J.C., Busemyer, J.R., Altmaier, E.M., \& Paulsen, J.S. (2008). Neurocognitive deficits related to poor decision making in people behind bars. Psychonomic Bulletin and Review, 15(1), 44-51.

Zelazo, P. D., \& Müller, U. (2002). Executive function in typical and atypical development. In U. Goswami (Ed.), Handbook of Childhood Cognitive Development (pp. 445-469). Oxford: Blackwell.

Zouk, H., Tousignant, M., Seguin, M., Lesage A., \& Turecki, G. (2006). Characterization of impulsivity in suicide completers: clinical, behavioral and psychosocial dimensions. Journal of Affective Disorders, 92(2-3), 195-204. 
Zuckerman, M. (1994). Behavioural Expressions and Biosocial Bases of Sensation-Seeking. Cambridge: Cambridge University Press. 


\section{Appendix 1}

\section{Pre-treatment information sheet for STURP participants}

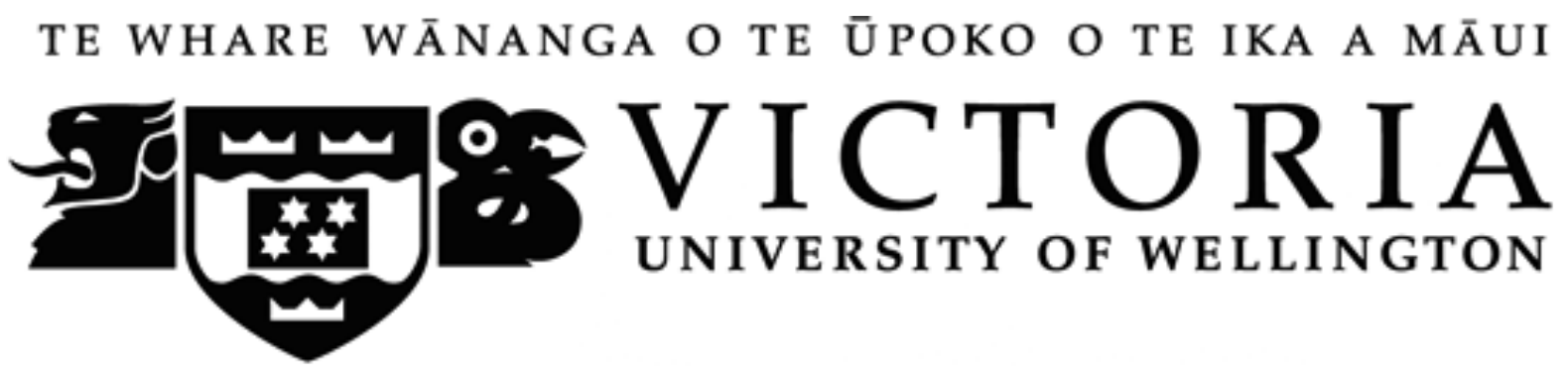

\section{Information About Research Project on Problem-Solving and Decision- Making}

\section{Who is doing this research?}

This research is being carried out by Bec Bell and Dr Devon Polaschek. Bec Bell is a $\mathrm{PhD}$ Student at Victoria University of Wellington and Dr. Polaschek is her research supervisor. This research is independent of the Department of Corrections. If you decide to take part, no one from the Department of Corrections will see your answers, it will not affect how the rest of your sentences goes, how the staff here manage you or the conditions under which you are released. No information will be given to the VPU programme staff, or custody staff, unless you tell me today that are about to go out after the session and seriously harm yourself or someone else today. If you tell me this, I am ethically required to warn someone here at the prison so that everyone is safe. This is the only exception to confidentiality.

\section{Why are we doing this research?}

We want to learn more about how men in prison think through problems and make decisions. We are also interested in whether there are changes after doing a rehabilitation programme. You have been especially chosen to talk to today because you are about to start a rehabilitation programme.

\section{What happens if you agree to take part?}

If you agree to take part, we will ask you to come along to two sessions. Each session will take about an hour-and-a-half. The first session will be today, and the second one will be in about seven months time, when you finish the programme. The reason we ask you to come to two sessions is so we can look at changes over time.

At the beginning of each session, we will tell you what we would like you to do, and if you are happy to take part, we will ask you to sign a form saying that. If you don't want to take part, you simply don't sign the form.

Saying yes to the first session does not mean that you have to take part in the next session, though we would like you to. When we come back the second time, we will ask you again for your permission to take part and you will sign another form, if you agree. 
If you agree to take part today but then decide part way through that you don't want to carry on, you can just tell Bec. If you do change your mind about today's session, we will destroy any information that you have given to us and you will not be included in the research project.

\section{Information about today}

If you agree to take part, there are five things that we would like you to do today; some of the tasks are done on the computer and some are done using pen and paper. It will take just over an hour to complete them all. We'll have a break in between tasks so you don't get too tired. Even though you might not get the tasks in this order, the first task is a gambling game. Using the computer, you get to choose cards from four different decks and try and win as much money as you can. The second and third tasks are tests to see how fast you are at pressing a button when you see a picture on the computer screen and how good you are at not pressing the button when the picture changes or you hear a 'STOP' warning. The fourth and fifth task is a challenge to see how good you are at blocking out distractions when you are trying to concentrate.

We would also like permission to look at your prison records. Bec Bell would like to record some information from your file that will help her estimate your criminal risk. It is important you know that the risk estimate will not be given to you or to the Department of Corrections - if they want a risk rating, they have to make one themselves. Our risk rating is only for research purposes.

\section{What will happen to my answers?}

We will look after them very carefully. You will be given a special ID number that we make up for you. The computer does not know anything personal about you. It records your answers just as a bunch of numbers in a file, along with your ID number. The information Bec takes from your file will also be identified only by ID number. All your information will be kept in a secure place at the university.

Because Bec Bell and Dr Polaschek have access to the rehabilitation programme evaluation database, we may put the information you give us here together with some of that information, if it looks like it could help answer some important questions. Your answers are very valuable. Bec Bell and Dr Polaschek will keep your information for as long as they can, if it can be used in other studies. But only the overall grouped results will ever be made available to the Department, or made public, NOT your personal results.

If the research goes well it will be published in a scientific journal and we will talk about it at professional conferences. You will know that you contributed to an important study that helped us know more about men in prison, and how to help them. But no one else will be able to tell that you took part.

\section{Want to know about the final outcome of this research?}

One of the interesting things about taking part in research is hearing how it came out. So, when we have finished the whole study we would like to send you a written summary of 
the results, some time in 2012. At the beginning of the second session, we will ask you to give us an address where we could send a summary if you'd like to get it.

In the meantime, please don't talk about this research with other people in the unit. If you do, it could spoil our results and the answers we have will not be as useful in helping us improve rehabilitation programmes.

Thanks for taking the time to read and hear about this research. It has important implications, and we hope you will seriously consider participating in it. 


\title{
Appendix 2 \\ Pre-treatment consent form for STURP participants
}

\author{
VICTORIA UNIVERSITY OF WELLINGTON \\ Te Whare Wananga o te Upoko o te Ika a Maui
}

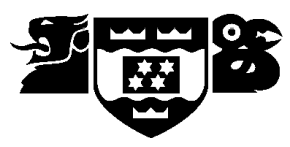

\section{Statement of Consent to Participate in}

\section{Session 1 of the Problem-Solving and Decision-Making Study}

I have read and understood the information sheet about this study. I have asked any questions I wanted to ask, and I am happy with the answers.

I agree to take part in this research I give my permission for the researchers to use the information I provide, for the purposes mentioned on the information sheet.

\section{Name:}

Signature:

Date: 
Appendix 3

Post-treatment information sheet for STURP participants

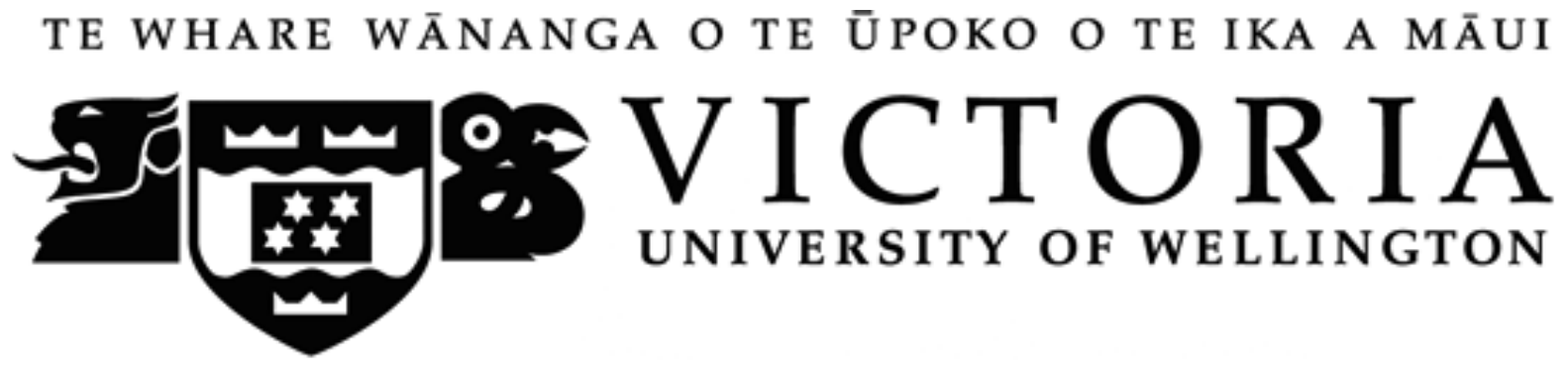

\section{Information About Research Project on Problem-Solving and Decision- Making}

Congratulations on finishing the programme! Now we would like to invite you to take part in the second session of our study, where we look at what's changed since you started the programme. But first, a quick reminder about the study...

\section{Who is doing this research?}

This research is being carried out by Bec Bell and Dr Devon Polaschek. Bec Bell is a PhD Student at Victoria University of Wellington and Dr. Polaschek is her research supervisor. This research is independent of the Department of Corrections. If you decide to take part, no one from the Department of Corrections will see your answers, it will not affect how the rest of your sentences goes, how the staff here manage you or the conditions under which you are released. No information will be given to the rehabilitation programme staff, or custody staff, unless you tell me today that are about to go out after the session and seriously harm yourself or someone else. If you tell me this, I am ethically required to warn someone here at the prison so that everyone is safe. This is the only exception to confidentiality.

\section{Why are we doing this research?}

We want to learn more about how men in prison think through problems and make decisions. We are also interested in whether this changes after doing a rehabilitation programme. You have been especially chosen to talk to today because you have now finished the rehabilitation programme and you participated last time, before you started the programme.

\footnotetext{
About today

Last time we met, you did several things using the computer and on pen and paper. They included a gambling game, some challenges to see how fast you reacted to pictures on the computer screen and how good you were at stopping a response, and some tasks to see how good you were at ignoring distractions. Today, we would like you to do these things again, to see if there are any changes. If you agree, it will take about an hour-and-a-half including time for a break in the middle.
} 


\section{What will happen to my answers?}

As we told you before, we will look after them very carefully. You have a special ID number. The computer does not know anything personal about you. It records your answers just as a bunch of numbers in a file, along with your ID number. The consent form that you sign to say that you agree to take part, will be kept at the university under lock and key, in a separate place from your answers. A copy of the consent form will go on your prison file, so that the prison can be sure that you gave your free consent to be part of the study.

Because Bec Bell and Dr Polaschek have access to the rehabilitation programme evaluation database, we may put the information you give us here together with some of that information, if it looks like it could help answer some important questions. Your answers are very valuable. Bec Bell and Dr Polaschek will keep your information for as long as they can, if it can be used in other studies. But only the overall grouped results will ever be made available to the Department, or made public, NOT your personal results.

If the research goes well it will be published in a scientific journal and we will talk about it at professional conferences. You will know that you contributed to an important study that helped us know more about men in prison for violence, and how to help them. But no one else will be able to tell that you took part.

\section{Want to know about the final outcome of this research?}

One of the interesting things about taking part in research is hearing how it came out. So, when we have finished the whole study we would like to send you a written summary of the results, some time in 2012. In this session, we will ask you to give us an address where we could send a summary if you'd like to get it. In the meantime, please don't talk about this research with other people in the unit. If you do, it could spoil our results and the answers we have will not help us improve rehabilitation programmes.

Thanks for taking the time again to read and hear about this research. It has important implications, and we hope you will seriously consider participating in this final part. 


\title{
Appendix 4 \\ Post-treatment consent form for STURP participants
}

\author{
VICTORIA UNIVERSITY OF WELLINGTON \\ Te Whare Wananga o te Upoko o te Ika a Maui
}

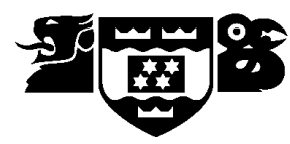

\section{Statement of Consent to Participate in}

\section{Session 2 of the Problem-Solving and Decision-Making Study}

I have read and understood the information sheet about this study. I have asked any questions I wanted to ask, and I am happy with the answers.

I agree to take part in this research I give my permission for the researchers to use the information I provide, for the purposes mentioned on the information sheet.

\section{Name:}

\section{Signature:}

\section{Date:}

When we have finished the whole study we would like to send you a written summary of the results, some time in 2012. If you will still be in prison then, remember that other inmates and staff may get to see your mail. You can give us the address of someone outside the prison who we can send this summary to if you would prefer.

Address for summary to be sent to: 


\section{Appendix 5 \\ Information sheet for student participation in Stop Signal task}

Rebecca Bell

PhD Student

Email: Rebecca.Bell@vuw.ac.nz

Phone: 463-6624
Dr Devon Polaschek, PhD

Associate Professor

Email: Devon.Polaschek@vuw.ac.nz

Phone: 463-5768

\section{What is the purpose of this research?}

- This research allows us to examine how quickly people respond to stimuli and how good people are at stopping a response when it's no longer appropriate. The research will also allow us to investigate how people typically act and think in different situations.

Who is conducting the research?

- Rebecca Bell is a PhD student. Dr. Polaschek is supervising this project. This research has been approved by the School of Psychology Human Ethics Committee under delegated authority of Victoria University of Wellington's Human Ethics Committee.

What is involved if you agree to participate?

- If you agree to participate in this study you will play a reaction time game on the computer. In the game, a square or circle will appear on the computer screen. When you see a shape all you have to do is hit a response button as quickly as you can. Sometimes though an auditory tone will follow the shape and when that happens, you have to try and stop yourself from pressing the button. After the game you will complete two short questionnaires.

- We anticipate that your total involvement will take no more than 30 minutes.

- During the research you are free to withdraw, at any point before your data have all been collected.

Privacy and Confidentiality

- We will keep your consent forms and data for five years from now.

- You will never be identified in my research project or in any other presentation or publication. The information you provide will be coded by number only.

- In accordance with the requirements of some scientific journals and organisations, your coded data may be shared with other competent researchers.

- Your coded data may be used in other, related studies.

- A copy of the coded data will remain in the custody of Rebecca Bell in Devon Polaschek's secure academic lab.

What happens to the information that you provide?

- The data you provide may be used for one or more of the following purposes:

- The overall findings may be submitted for publication in a scientific journal, or presented at scientific conferences.

- The overall findings may form part of a $\mathrm{PhD}$ Thesis, Masters Thesis, or Honours research project that will be submitted for assessment.

If you would like to know the results of this study, please provide your e-mail address on the consent form. The results will be available approximately March, 2013.

If you have any further questions regarding this study please contact any one of us above. 


\title{
Appendix 6 \\ Consent form for student participation in Stop Signal task
}

\begin{abstract}
Statement of consent
I have read the information about this research and any questions I wanted to ask have been answered to my satisfaction.

I agree to participate in this research. I understand that I can withdraw my consent at any time, prior to the end of my participation.
\end{abstract}

Name:

Signature:

Date:

StudentID:

Email address for results summary:

Copy to:

[a] participant,

[b] researcher (initial both copies below) 


\section{Appendix 7 \\ Student debriefing sheet for participation in Stop Signal task}

\section{Thank you for participating in this experiment}

This study examined how quickly people respond to stimuli and how good they are at stopping a response that is no longer appropriate, otherwise known as inhibition. This study also examined how people typically act and think in different situations.

Research has shown that being able to inhibit responses that are no longer appropriate is an important skill for controlling our behaviour (e.g., Bechara, 2005). In my research, I am investigating how the general population performs in a task of inhibition; the computer game you played today.

Another part of my research involved administering the same computer game you completed today to a group of male, high-risk offenders. The computer game is a novel way of assessing impulse control in a forensic setting. Using this novel method, I wanted to investigate the magnitude of high-risk offenders' impulse control problems in comparison to the general, non-offender population. To answer that question, I need to match offender performance to non-offender performance on the computer game, which is why I recruited you today.

As well as the computer game, you completed two questionnaires related to impulse control. Another part of my research is to compare questionnaire scores with scores from the computer game to examine the utility of different measurement methods of impulse control.

This kind of research is very important to researchers and practitioners involved in the assessment and rehabilitation of offenders. Understanding more about what causes impulsive behaviour and how best to assess those deficits advances our understanding about how best to help offenders reduce their risk of re-offending in the future. 


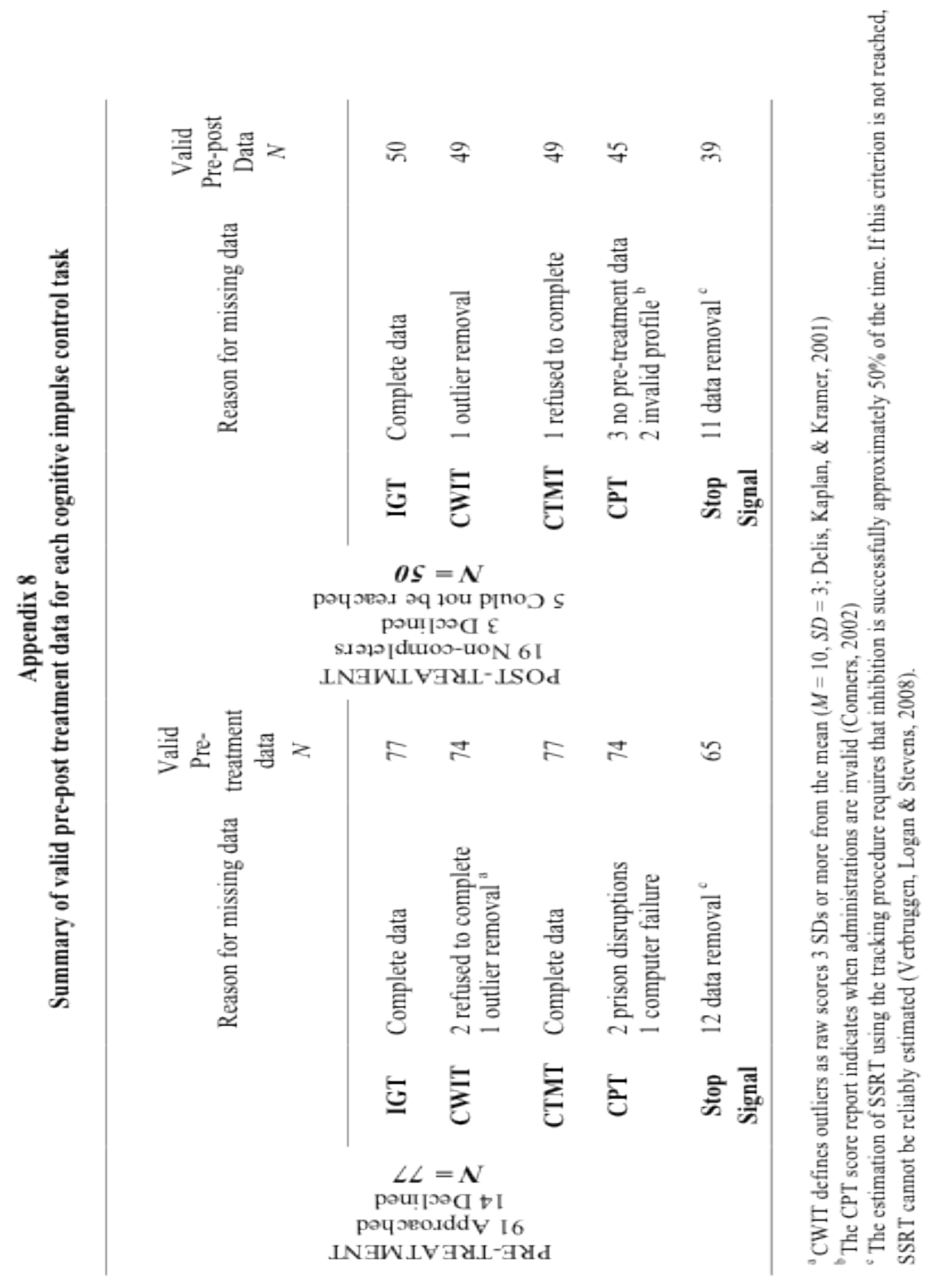

